\title{
Theory and Simulation of the Physics of Space Charge Dominated Beams
}

\author{
Final Progress Report
}

Submitted to:

Chicago Operations Office

Department of Energy

Submitted by:

Plasma Physics Division

Naval Research Laboratory

Washington, DC 20375-5346

Principal Investigator:

Dr. Irving Haber

301-405-4962

haber@glue.umd.edu

NRL Contact:

Dr. Sidney Ossakow

202-767-2723

ossakow@ccs.nrl.navy.mil

Interagency Agreement: DE-AI02-93ER40799

Date:

December 31, 2002

DOE Patent Clearance Granted

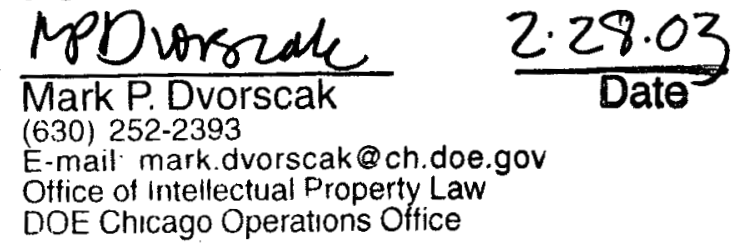




\section{DISCLAIMER}

This report was prepared as an account of work sponsored by an agency of the United States Government. Neither the United States Government nor any agency Thereof, nor any of their employees, makes any warranty, express or implied, or assumes any legal liability or responsibility for the accuracy, completeness, or usefulness of any information, apparatus, product, or process disclosed, or represents that its use would not infringe privately owned rights. Reference herein to any specific commercial product, process, or service by trade name, trademark, manufacturer, or otherwise does not necessarily constitute or imply its endorsement, recommendation, or favoring by the United States Government or any agency thereof. The views and opinions of authors expressed herein do not necessarily state or reflect those of the United States Government or any agency thereof. 


\section{DISCLAIMER}

Portions of this document may be illegible in electronic image products. Images are produced from the best available original document. 


\section{Theory and Simulation of the Physics of Space Charge Dominated \\ Beams}

\section{FINAL PROGRESS REPORT}

\section{Introduction}

A long history of progress in understanding the novel nonlinear physics that is fundamental to understanding the transport of space-charge-dominated beam has resulted from the current program. This progress has largely resulted from a continuing series of detailed comparisons between particle-in-cell (PIC) simulations and experimental observation primarily at the University of Maryland. These comparisons were begun initially to augment understanding of the basic nonlinear physics of space-chargedominated transport necessary to achievement of the objective of the Heavy Ion Fusion (HIF) research effort, the simulation effort was largely centered at the Naval Research Laboratory (NRL). The scope of this research was expanded to meet the need for increased luminosities in a variety of accelerator systems. This expansion in scope has occurred largely as a result of the program to construct the University of Maryland Electron Ring (UMER) which, by virtue of the ring geometry, will permit access to the physics of a space charge dominated beam propagating over long distances. Though the program reported on here was terminated, the research is continuing at the University of Maryland. Some of the publications included therefore include research performed at NRL but reported under the continuation of the program.

Early work on the physics of space-charge-dominated beams was centered on fundamental questions of equilibrium and stability in beam transport. A significant result of this work was the identifying the importance of space-charge collective modes in describing beam behavior ${ }^{1,2}$ and the utility of this modal description in explaining experimental observation. A difficult and as yet unresolved issue is determination of the mechanism by which the observed collective modes are damped as they propagate along with the beam. A significant observation in the simulations is that though modes with shorter transverse wavelengths appear to be damped relatively quickly, the modes with transverse variation comparable to the beam diameter can propagate relatively undamped. 
These modes have been observed to propagate over hundreds of alternating-gradient lattice periods without significant damping. 


\section{WARP Simulations of UMER}

In view of the relatively long propagation lengths over which collective phenomena occur, it is difficult to examine questions relevant to steady state transport without access to experiments that are longer than those currently available. The scaled electron approach employed in the experiments at IREAP is specifically targeted at investigating the evolution of a space-charge-dominated beam over distances measured in hundreds of focusing periods, so that the physics is no longer dominated by the characteristics of the beam source. Even for a scaled electron experiment, construction of a linear device with a length measured in the hundreds of periods required to investigate long-timescale transverse and longitudinal beam dynamics, would require a relatively large and complex apparatus. An alternative approach is construction of a ring. This is the path taken by the UMER project at the University of Maryland. ${ }^{3,4}$ While a ring is a convenient way of accessing the long beam lifetimes necessary to study long-time-scale space-charge-dominated beam physics, the bending of a space-charge-dominated beam is not yet well-explored. Fortunately, the dynamics of a space-charge-dominated beam in a circular system is of substantial interest for its own sake, so that the complications associated with the construction, operation, and simulation of a circular system also carry a set of payoffs that result directly from interest in the circular geometry. Because of the novelty of the physics accessed by this machine, including the lack of any previous operation of such a space-charge-dominated ring, UMER is a fertile area for innovative experimental and theoretical research.

Propagation over the long distances is required for experimental investigation of the nature of steady state transport. Because internal correlations can strongly influence the evolution of a space-charge-dominated beam, to investigate the evolution to steady state it also important to fully characterize the beam distribution function rather than just averaged beam quantities. The diagnostics built into UMER have, therefore, been explicitly designed to measure the details of the evolution of the full beam distribution that are necessary for comparison to theory and simulation, and not just the averaged beam characteristics such as emittance, rms envelope and total current. For example, these diagnostics include various apertures that can be rotated into the beam at the gun output as well as a pepper pot that is part of the main diagnostic chamber. These 
apertures can be used to infer information that can be used along with simulation, as discussed below, to infer important characteristics of the beam distribution. An additional sophisticated diagnostic that is under development is a retarding energy analyzer that can be used to measure the time resolved longitudinal distribution function. ${ }^{5}$ This diagnostic is particularly significant not only because of its uniqueness, but also because it will provide access to measurement of longitudinal physics that to date has been hardly explored. In addition, ability to benchmark longitudinal measurements against simulation are an important objective towards a credible simulation capability of the UMER experiment.

Recent simulation work has concentrated on comparisons to experimental data as it is obtained, as well as on studying the physics of a space-charge-dominated beam with emphasis on issues relevant to the design and construction of the UMER ring. ${ }^{6}$ Excellent agreement has, in fact, been obtained in detailed comparisons between experimental observation and simulation in the injector section, ${ }^{7}$ as well as the first ring sections. ${ }^{8,9,10}$

Downstream measurements of the beam characteristics, while they can be reproduced by appropriate choice of initial distribution, have displayed sufficient sensitivity to assumed details of the initial distribution to highlight the importance of obtaining first-principles predictions of the detailed beam characteristics. Because of the difficulty of resolving the cathode-grid structure and the electron gun structure in a single calculation simulations have been performed to examine sensitivity of the downstream beam characteristics to assumptions about the consequences of the perturbation to the beam introduced by the cathode grid.

Much work has been done, some of it spanning several decades, to design particle beam sources. Emphasis in this work has, however, historically has emphasized predicting the total current emerging from the source. More recently attention has been shifted toward also predicting other averaged beam quantities such as the rms radius or rms emittance. However, the beam emerging from the source is not generally in detailed local equilibrium with the applied focussing forces. This deviation from local equilibrium will, in general launch collective oscillations that can persist for long distances. The fundamental properties of predicting beam propagation over long distance therefore depend on the excitation of collective modes in the source region, and 
predicting the details of this excitation depends on understanding the full beam

distribution function. Recent work has therefore emphasized full characterization of source distribution function. ${ }^{11}$ From recent pepper pot measurements of the distribution near the gun output it was determined that the grid perturbation could actually impart a double-humped velocity distribution when the grid to cathode potential was sufficiently large. Simulations were then used to infer the emittance and the likely characteristics of the beam distribution that were simultaneously consistent with several independent measurements. ${ }^{12,13}$ While it is still important to do simulations of the grid cathode region, especially, as discussed below, because of the importance of such simulations in understanding the longitudinal velocity spread, the agreement that has been obtained is important in establishing the importance of the detailed characterization of the functional dependence of the beam distribution on the value of the cathode grid potential.

\section{Physics of Space-Charge-Dominated Beams}

UMER can be said to be a modern accelerator experiment in the sense that there has been a great deal of reliance on computer simulation during the design phase, especially with regard to understanding the influence of the intense space charge on the beam dynamics. A major contribution of the UMER project is therefore the demonstration of what is required to successfully utilize computer simulations to reliably design space-charge-dominated accelerators. An example of an accelerator system that relies on such a capability for its ultimate success, is the space-charge-dominated linac system under development for the production of economical power from heavy-ionbeam ignition of a thermonuclear pellet. The UMER research program is therefore being conducted in close collaboration with personnel at the Heavy Ion Fusion Virtual National Laboratory (HIF - VNL). An example of the results from this collaboration has been the use of numerical needs identified in the UMER simulations to promote changes in the WARP simulation capability. The changes that are then made are then available for use on UMER as well as in the HIF program. ${ }^{14}$

Experience in using the simulations to predict behavior expected from the HIF experiments ${ }^{15}$ is also a shared research effort where the combination of the two viewpoints allows a refinement of techniques not likely to occur from a single 
perspective. $^{16}$ These refined techniques are an important part of plans for future experiments and the joint capability to exploit simulations to design future experiments is an integral part of the HIF research program. ${ }^{17,18,19}$

On a more fundamental level, the physics associated with the UMER experiment has been examined to determine the conditions under which the individual particle orbits exhibit chaotic behavior. ${ }^{20,9}$ Not only is study of the onset of chaos an area of importance in accelerator dynamics, but the studies to date have indicated the possible that the dynamics in UMER might be used as a model to study the onset of dynamical chaos in galactic systems.

1. I. Haber, A Friedman, D. P. Grote, S. M. Lund, S. Bernal, R. A. Kishek, "Recent Progress in Heavy Ion Fusion Simulations," Nucl. Instr. and Meth, in Phys Res. A 464, 343 (2001), manuscript is included for reference.

2. R. A. Kishek, S. Bernal, P. G. O'Shea, M. Reiser, I. Haber, "Transverse SpaceCharge Modes in Non-Equilibrium Beams," Nucl. Instr. and Meth, in Phys Res. A 464, 484 (2001), manuscript is included for reference.

3. P. G. O'Shea, M. Reiser, R. A. Kishek, S. Bernal, H. Li, M. Preussner, V. Yun, Y. Cui, W. Zhang, Y. Zou, T. Godlove, D. Kehne, P. Haldemann, I. Haber, "The University of Maryland Electron Ring," Nucl. Instr. and Meth, in Phys Res. A, 464, 646 (2001), manuscript is included for reference.

4. P. G. O'Shea, B. Beaudoin, S. Bernal, D. Feldman, M. Glanzer, T. Godlove, J. Harris, M. Holland, H. Li, R. A. Kishek, B. Quinn, N. Rashimi, M. Reiser, A. Valfells, M. Virgo, M. Walter, R. Yun, V. Yun, D. Kehne, I Haber, "The University of Maryland Electron Ring (UMER)," Proc. 2001 Particle Accelerator Conf., manuscript is included for reference.

5. Y. Zou, Y. Cui, V. Yun, R. A. Kishek, S. Bernal, I. Haber, M. Reiser, P. G. O'Shea, J. G. Wang, "Compact High-Resolution Retarding Field Energy Analyzer for SpaceCharge-Dominated Electron Beams," Phys. Rev. ST 5 072801-1 (2002), manuscript is included for reference.

6. H. Li, S. Bernal, R. A. Kishek, I. Haber, Y. Zou, P. G. O'Shea, M. Reiser, "Simulation Studies on Matching os Space-Charge-Dominated Beams for the University of Maryland Electron Ring (UMER)," submitted to Nucl. Instr. And Methods A, manuscript is included for reference.

7. D. Kehne, T. Godlove, P. Haldemann, S. Bernal, S. Guharay, R. Kishek, Y. Li, P. O'Shea, M Reiser, V. Yun, Y. Zou, I. Haber, "Injector for the University of Maryland Electron Ring (UMER)," Nucl. Instr. and Meth, in Phys Res. A 464, 605 (2001), manuscript is included for reference. 
8. P. G. O'Shea, S. Bernal, R. A. Kishek, M. Reiser, T. Godlove, A. Valfells, "Experiments with Space Charge Dominated Beams for Heavy Ion Fusion Applications," accepted for publication in Laser and Particle Beams, manuscript is included for reference.

9. R. A. Kishek, S. Bernal, C. L. Bohn, I. Haber, H. Li, P. G. O'Shea, M. Reiser, M. Walter, "Simulations and Experiments with Space-Charge-Dominated Beams," submitted to Phys. Plasma, manuscript is included for reference.

10. S. Bernal, B. Beaudoin, Y. Cui, M. Glanzer, T. F. Godlove, J. Harris, M. Holloway, I. Haber, R. A. Kishek, W-T. Lee, H. Li, D. Lamb, B. Quinn, M. Quirus, M. Resier, A. Valfells, M. Walter, R. Yun, P. G. O'Shea, "Intense Beam Transport Experiments in a Multi-Bend System at the University of Maryland Electron Ring (UMER)," submitted to Nucl. Instr. and Methods A, manuscript is included for reference.

11. I. Haber, S. Bernal, R. A. Kishek, P. G. O'Shea, M. Reiser, A. Valfells, D. P. Grote, "Computer Simulation of the UMER Electron Gun," Proc. 2001 Particle Accelerator Conf, manuscript is included for reference.

12. I. Haber, S. Bernal, F. M. Bieniosek, C. M. Celata, A. Friedman, D. P. Grote, E. Henestroza, W. B. Herrmannsfeldt, R. A. Kishek, P. G. O'Shea, M. Reiser, J.-L. Vay, "End-to-End Simulation: The Front End," J.-L. Vay, accepted for publication in Laser and Particle Beams, manuscript is included for reference.

13. I. Haber, S. Bernal, C. M. Celata, A. Friedman, D. P. Grote, R. A. Kishek, B. Quinn, P. G. O'Shea M. Reiser, J.-L. Vay, "Collective Space-Charge Phenomena in the Source Region," submitted to Nucl. Instr. And Methods A, manuscript is included for reference.

14. D. P. Grote, A. Friedman, G. D. Craig, W. M. Sharp, I. Haber, "Progress Toward Source-to-Target Simulation," Nucl. Instr. and Meth, in Phys Res. A, 464, 563 (2001), manuscript is included for reference.

15. C. M. Celata, D. P. Grote, I. Haber, "PIC Simulations of Beam Dynamics Experiments on the High Current Experiment," Proc. 2001 Particle Accelerator Conf., manuscript is included for reference.

16. C. M. Celata, F. M. Bieniosek, E. Henestroza, J. W. Kwan, E. P. Lee, G. Logan, L. Prost, P. A. Seidl, J.-L. Vay, W. L. Waldron, S. S. Yu, JJ. Barnard, D. A. Callahan, R. H. Cohen, A. Friedman, D. P. Grote, S. M. Lund, A. Molvik, W. M. Sharp, G. Westenskow, Ronald C. Davidson, Philip Efthimion, Erik Gilson, L. R. Grisham, Richard Majeski, Hong Qin, Edward A. Startsev, S. Bernal, Y. Cui, D. Feldman, T. F. Godlove, I. Haber, J. Harris, R. A. Kishek, H. Li, P. G. O'Shea, B. Quinn, M. Reiser, A. Valfells, M. Walter, Y. Zou, D. V. Rose, D. R. Welch, "Progress in Heavy Ion Fusion Research," submitted to Phys. Plasma, manuscript is included for reference.

17. J. J. Barnard, L. E. Ahle, R. O. Bangerter, F. M. Bieniosek, C. M. Celata, A. Faltens, A. Friedman, D. P. Grote, I. Haber, E. Henestroza, M. J. L. de Hoon, V. P. Karpenko, R. A. Kishek, J. W. Kwan, E. P. Lee, B. G. Logan, S. M. Lund, W. R. Meier, A. W. Molvik, T. C. Sangster, P. A. Seidl, W. M. Sharp, "Planning for an Integrated Research Experiment," Nucl. Instr. and Meth, in Phys Res. A, 464, 621 (2001), manuscript is included for reference. 
18. P. A. Seidl, R. Bangerter, C. Celata, A. Faltens, V. Karpenko, E. Lee, I. Haber, S. Lund, A. Molvik, "Overview of the Scientific Objectives of the High Current Experiment for Heavy-Ion Fusion Proc. 2001 Particle Accelerator Conf

19. J. J. Barnard, L. E. Ahle, D. Baca, R. O. Bangerter, F. M. Bieniosek, C. M. Celata, E. Chacon-Golceher, R. C. Davidson, A. Faltens, A. Friedman, R. M. Franks, D. P. Grote, I. Haber, E. Henstroza, M. J. L. de Hoon, I. Kaganovitch, V. P. Karpenko, R. A. Kishek, J. W. Kwan, E. P. Lee, B. G. Logan, S. M. Lund, W. R. Meier, A. Molvik, C. Olson, L. R. Prost, H. Qin, D. Rose, G-L. Sabbi, T. C. Sangster, P. A. Seidl, W. M. Sharp, D. Shuman, J. L. Vay, E. L. Waldron, D. Welch, S. S. Yu, "Progress in Heavy Ion Driven Inertial Fusion Energy: From Scaled Experiments to Integrated Research Experiment," Proc. 2001 Particle Accelerator Conf., manuscript is included for reference.

20. R. A. Kishek, C. L. Bohn, I. Haber, P. G. O’Shea, M. Reiser, H. Kandrup, "Computational Investigation of Dissipation and Reversibility of Space-Charge Driven Processes in Beams," Proc. 2001 Particle Accelerator Conf., manuscript is included for reference. 


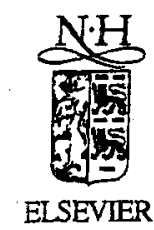

Nuclear Instruments and Methods in Physics Research A 464 (2001) 343-350

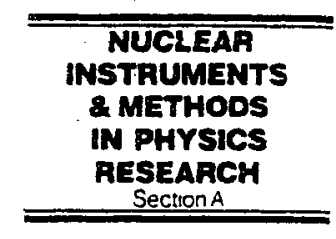

www.elsevier.ni/locate'nima

\title{
Recent progress in heavy ion fusion simulations ${ }^{\text {th }}$
}

\author{
I. Haber ${ }^{\mathrm{a} *}$, A. Friedman ${ }^{\mathrm{b}}$, D.P. Grote ${ }^{\mathrm{b}}$, S.M. Lund ${ }^{\mathrm{b}}$, S. Bernal ${ }^{\mathrm{c}}$, R.A. Kishek ${ }^{\mathrm{c}}$ \\ a Plasma Physics Division, Code 4790, Naval Research Laboratory, 4555 Overlook Ave., S.W., Washington, DC 20375, USA \\ ${ }^{b}$ Heavy Ion Fusion Virtual National Laboratory, Berkeley, CA 94720, USA \\ 'Institute for Plasma Research, University of Maryland, College Park, MD 20742, USA
}

\begin{abstract}
Based on the success that simulations have enjoyed in reproducing both theory and experimental measurement, simulation methods have become increasingly accepted for studying the physics of space-charge-dominated beams. Such studies have recently demonstrated the utility of viewing the evolution of space-charge-dominated beams in terms of bounded collective warm-plasma oscillations. Viewing the plasma in this way has not only resulted in simplifying the expianation of experimental data but has also opened the possibility of utilizing th excitation of these modes to tailor space charge dominated beams for optimizing performance of heavy ion fusion drivers. (C) 2001 Elsevier Science B.V. All rights reserved.
\end{abstract}

PACS: $29.27 . \mathrm{Bd} ; 29.27 . \mathrm{Eg} ; 52.25 . \mathrm{Wz} ; 52.65 .+\mathrm{z}$

Keywords: Warm plasma; Collective oscillations; Particle-in-cell; Beam transport; Space charge; Simulation

\section{Introduction}

In recent years, particle-in-cell (PIC) numerical techniques have become a generally accepted tool for the simulation of space-charge-dominated beams. The WARP PIC/accelerator code [1], which was explicitly written for this purpose, has enjoyed extensive and successful benchmarking against experimental measurements, and has become a primary numerical tool, particularly for the induction linac approach to HIF drivers. Numerous other numerical tools are also being employed to study the beam physics in heavy-ion

\footnotetext{
Work supported by the US Department of Energy by NRI at $\mathrm{HI}$ VNL and at U. Md. under contracts DE-AIO293ER40799, DE-AI02-94ER54232, W-7405-ENG-48, and DEFG02-92ER54178.

"Corresponding author. Fax: +1-202-7670631.

E-mail address: haber@ppd.nrl.navy.mil (I. Haber).
}

fusion accelerators, and are reported in these proceedings, but will not be discussed here. In addition, because of the complexity of predicting the beam evolution in space-charge-dominated accelerator systems, a significant effort is also underway using WARP as a tool $[2,3]$ in the detailed design of future accelerators.

Nonlinearities in the self-consistent spacecharge forces have generally been found to play a significant role in the transport of space-chargedominated beams. In the absence of analytic tools which can follow this nonlinear behaviour adequately, simulation has come to play a dominant role in the design of space-charge-dominated transport systems as well as in the understanding of existing experiments. In order to adequately meet continuing simulation needs as space-chargedominated experiments increase in complexity, WARP is continually being upgraded [4] to 
increase the inciusiveness of the accelerator model as well as to benefit from the capabilities of modern parallel computer hardware.

While the numerical requirements for adequately performing simulations are well understood under most circumstances, and can usually be checked by varying numerical parameters until the physical results become independent of those parameters, agreement between simulation and experiment has not always been as close as expected. This has often motivated increases in the sophistication of the numerical model, such as incorporating into the code a more accurate representation of the focusing elements. A more common occurrence, however, has been the determination that differences between the simulation and experiment can be traced to an inadequately detailed specification of the initial beam distribution function.

As a result of the complexity possible in the evolution of even a relatively simple space-chargedominated beam, the issue of adequately specifying the initial distribution has been the subject of extensive study. Since this issue has proved important to a large class of simulations, the discussion below will deal with closely related issue of the internal degrees of freedom accompanying collective space-charge oscillations and how they influence beam evolution. Viewing the beam response in terms of these collective warm-plasma oscillations has enhanced understanding of the detailed requirements for adequately describing the beam and helped to improve detailed agreement between simulation and experiment.

\section{Space charge modes on a $K-V$ beam}

Analytic description of space-charge-dominated beam dynamics in the transverse direction has generally employed an initial Kapchinskij-Vladimirskij $(\mathrm{K}-\mathrm{V})$ distribution [5], which remains the single known analytical equilibrium of a spacecharge-dominated beam in an alternating-gradient transport system. The $\mathrm{K}-\mathrm{V}$ distribution, however, has the characteristic that all particles oscillate at the same betatron frequency, and the internal beam characteristics are highly correlated. The singular characteristic of this distribution has generally raised questions as to the applicability of results obtained from analysis of a $\mathrm{K}-\mathrm{V}$ distribution to more physical beam distributions.

The Vlasov analysis of modes on a beam in the presence of nonzero transverse kinetic energy became an active area of investigation after the discovery that some of the transverse normal modes $[6,7]$ were unstable, with growth times of just a few betatron periods. If growth of these modes, especially considering the rapid growth rates, would contribute to a substantial increase in beam emittance, this emittance increase could limit the transportable intensity and therefore might limit ability to focus the beam onto a fusion target. Furthermore, concern over instability-caused transport limits increased when analysis of these modes was extended to $\mathrm{K}-\mathrm{V}$ beams in alternatinggradient focusing systems and large regions in parameter space were found unstable [8].

A characteristic of the unstable eigenfunctions however, is that in the linear approximation for which they are derived, they identically preserve rms emittance. Consequently, the linear theory can not predict the extent to which these modes cause rms emittance growth. And it was found, by using self-consistent PIC simulations which include the nonlinear space-charge evolution, that these modes caused essentially no emittance growth for alternating gradient focusing systems with undepressed phase advances substantially below $90^{\circ}$ per lens period [8]. This lack of $\mathrm{rms}$ emittance growth was also found both for continuous solenoid focusing, as well as for interrupted solenoid transport even at $90^{\circ}$ phase advance. What was observed in these cases is merely an evolution of the initial distribution away from the initial $\mathrm{K}-\mathrm{V}$ state. It was therefore concluded that many of the instabilities predicted for an initial $\mathrm{K}-\mathrm{V}$ distribution were due primarily to the singular nature of the highly correlated initial distribution.

\section{Emittance growth calculation by conservation of energy}

A tool that has been found useful for estimating limits on emittance growth which are independent 
of the details of the distribution function, is to invoke conservation of energy in the transverse direction $[9,10]$. By calculating the change in potential energy between initial and final state, which is generally assumed to be transversely uniform, conservation of energy can then be used to calculate the change in beam kinetic energy. Furthermore, the interchange of potential to kinetic energy occurs rapidly, on a time scale generally of the order of a fraction of a plasma period [11].

One of the inadequacies inherent in this approach, is that in the presence of varying external fields, the beam frame energy is not necessarily conserved. Furthermore, the conservation of energy as a means of calculating beam evolution does not give insight into, possibly important, details of the beam dynamics. This will be discussed below. A practical limit, also discussed below, arises from a lack of detailed knowledge of the initial beam distribution. In particular, it has sometimes been observed that the beam initial distribution has transverse kinetic energy not easily observed experimentally and which can complicate estimation of total transverse energy in the beam system. Appreciation of the significance of not including this kinetic energy has arisen from difficulties that were encountered in explaining experimental observations.

\section{Experiment and simulation of the five beamlet experiment}

A detailed description of the many experiment/simulation comparisons which are relevant to the current discussion is beyond the scope of this paper. However, one early experiment which is illustrative, is the five beamlet experiment at the University of Maryland [12]. In this experiment, a low-energy electron beam was passed through a mask which carved the original beam into five beamlets, one in the center and four equally placed around the periphery. A primary reason for this experiment was to test the use of conservation of energy as a predictor of the emittance growth in an initially nonuniform beam. The final measured emittance, and the final emittance predicted from simulations did verify the value predicted from the conservation of energy calculation. However, complex internal structure was observed on a phosphor screen, moved along the beam axis to display variation in beam profile. This complex structure was also seen in the simulations, in close and detailed agreement with experimental observation. Particularly striking evidence for the complexity of the internal structure of the beam response was the observation of a downstream image of the original five-beamlet configuration. An additional interesting feature of the experiment was the low amplitude, long length (compared with the characteristic time for emittance growth) fluctuations in the rms emittance which were observed in the simulations. Because of the high degree of agreement between simulation and experiment it is reasonable to infer that these fluctuations also occur in the experiment.

Another interesting characteristic of the experimental data is that the initial beam cross section at the plane of the mask shows hollowing in the initial current distribution. This could be seen in the phosphor screen pictures and was verified by Faraday cup measurements of the current crosssection, which showed a 20 percent hollowing. Attempts to incorporate this hollowing into the simulated initial conditions were not successful in the sense that a hollow initial distribution in the simulation was found to consistently degrade the agreement with experiment. It was concluded that the observed differences in behaviour were likely to be a consequence of internal correlations in the initial beam distribution which were not being accounted for in the initial beam distribution used in the simulations.

The conclusion reached at the time of the five beamlet experiment (1991) was that a detailed understanding of the complex mechanism for image formation, an explanation of the long length rms emittance oscillations, and resolution of the inability to explain the influence of initial hollowing, would have to await a more comprehensive understanding of the internal dynamics of a space-charge-dominated beam. 


\section{Anisotropy as a source of emittance growth}

In a beam system which is anisotropic, in the sense that the beam kinetic temperatures or emittances are different in different directions, the anisotropy can cause instabilities which drive the beam distribution toward equipartition. Such a mechanism has been suggested as important for the short bunches in RF linacs [13-15]. A similar mechanism for driving the beam towards isotropy has also recently been identified as being operative when an anisotropy develops in the transverse direction as a space-charge-dominated beam is transported through a bend [16].

Such an anisotropy-driven instability is also possible in a beam which is long compared with its cross-section, when the transverse temperature substantially exceeds the longitudinal temperature $[17,18]$. This is a common situation in accelerators which results from the adiabatic cooling of the longitudinal beam temperature as the beam is accelerated from the emitting surface. It can also result from beam manipulations which cause an increase in the transverse temperature without increasing the longitudinal temperature.

Simulations performed of a beam long compared with the pipe radius, with an initial $\mathrm{K}-\mathrm{V}$ distribution in the transverse direction, and with the large anisotropy resulting from a very small initial longitudinal temperature, have been found in reasonable agreement with theoretical predictions [20] of growth rate and most unstable wavelength $[21,22]$. Of particular note, however, is that the general characteristics of the instability, including the growth rates, and the spectrum and structure of the modes, is similar when simulations are performed with a transverse and semi-Gaussian distribution which is uniform in configuration space and has a Gaussian velocity distribution with uniform temperature across the beam [19]. This observation that the electrostatic modes responsible for the isotropization instability are similar in both $\mathrm{K}-\mathrm{V}$ and semi-Gaussian distributions, and are unstable in both cases, suggests that the predictions of the $\mathrm{K}-\mathrm{V}$ analysis have generality beyond what might have been expected from earlier findings that instability predicted from
$\mathrm{K}-\mathrm{V}$ theory were not valid for more physical distributions.

\section{Warm-plasma collective modes}

Combined evidence exists from both simulation and experiment of the importance of space charge collective behavior in the warm plasmas that describe most space-charge-dominated beams. Even though the $\mathrm{K}-\mathrm{V}$ theory has been found to predict growth which is apparently a consequence of the singular nature of the $\mathrm{K}-\mathrm{V}$ distribution, and which can not be extended to more physical distributions, this does not necessarily imply that the oscillatory behavior, i.e. the real part of the eigenvalues, is not relevant to non- $\mathrm{K}-\mathrm{V}$ distributions. Furthermore, it appears that when there is a mechanism for transferring substantial energy into the collective modes characteristic of the beam, such as the driven breathing motion in an alternating-gradient focusing system or the energy in an anisotropic distribution, the even unstable behavior predicted by the $\mathrm{K}-\mathrm{V}$ theory can be relevant to other distributions.

The relevance of the $\mathrm{K}-\mathrm{V}$ results is not primarily justification of the correctness of using a $K-V$ distribution to describe a beam. Rather, the recognition that warm-plasma oscillations are likely to be excited in a number of beam systems, leads to a useful way of interpreting both simulation results and experimental data. The possibility is also raised of exciting these oscillations to control the evolution of a space-chargedominated beam.

The transverse warm-plasma eigenmodes are characterized by an interchange between kinetic and potential energy. Therefore, at one phase a single mode can consist entirely of a density perturbation and a quarter of a period later the mode will appear only as an inhomogeniety in beam kinetic temperature, or pressure. Similarly, the density perturbation can go from hollowed to peaked in haif a period. This behavior was observed in both experiment and simulation on the large ion source experiment at LBNL [23] as well as in experiments and simulation on the prototype injector experiment [24] at the University of 
Maryland. If a measurement of the beam density is made at a single plane, and there is little transverse density perturbation because of the phase of a large transverse mode at the measurement plane, it would be possible to conclude that the beam is not transversely perturbed even in the presence of such a large amplitude oscillation. In a similar way, as was observed on the LBNL source, a hollow beam at the measurement plane can be a result of a peaked distribution upstream. This type of misinterpretation of data has been a frequent result of the inability to place diagnostics close enough to the emitter surface, or the inability to get sufficiently accurate upstream data.

In terms of the interpretation of experimental data from the five beamlet experiment as discussed above, this means that the initial hollowing should be interpreted as a particular phase in a transverse oscillation which may have a substantial variation in pressure across the beam. This can give rise to downstream behavior somewhat different from what was observed using the initial conditions which were assumed in attempting to incorporate hollowing into the simulations. Also the wave picture is a useful way of viewing the image formation since the initial disturbance caused by masking the beam can be viewed as launching a series of large amplitude collective oscillations with quadrupole symmetry. If these oscillations are similar enough in period so that they are nearly in phase after half of an oscillation, they will form an image of the original configuration. Since the individual modes, after they have separated, can be of small enough amplitude so as to not strongly alter the rms emittance, the small oscillations in rms emittance observed in the simulation as the beam propagates can also be a result of some nonlinear addition of any remaining oscillations. And these modes appear to be slowly damped.

A particularly striking example of the transverse wave-like behavior which can occur in a space charge-dominated beam was seen in the phosphor screen measurements on the prototype injector experiment at the University of Maryland [25]. By using a small circular mask to exclude all but the relatively uniform center of the beam, the simulation could be conducted with a known initial distribution. Observed experimentally, and in close agreement in the simulations, is the formation of a ring of charge at the outer edge of the beam which then propagates into the center and back outward until it eventually loses its coherence.

Since a beam is usually born on an emitting surface which ends abruptly, some similar behavior to what is observed in this experiment is likely to be present almost universally in space-chargedominated beams, although the amplitude of the initial perturbation, as well as the time scale for propagation into the beam, depend on the initial beam emittance, so that the effect might be small in some experiments. It is also worth noting that this effect does not strongly affect the transverse rms emittance. This is predicted by calculations made by invoking energy conservation, as discussed above, which predict [10] only a small decrease (of order a few percent) in rms emittance for this configuration.

In addition to the experimental and simulation evidence supporting the concept of warm plasma oscillations as a significant mechanism for describing the internal behavior of a space charge dominated beam, strong theoretical support has come from the derivation [26] using a warm-fluid description, of many of the modes which were originally derived using a Vlasov description and an initial $\mathrm{K}-\mathrm{V}$ distribution. It should be noted that only the high frequency modes, which are not unstable, are recovered in this formalism. However, more recently, the warm fluid approach was also used to describe unstable behavior of collective modes in the presence of a temperature anisotropy [27] which demonstrates that even some of the unstabie behavior originally described using the Vlasov- $\mathrm{K}-\mathrm{V}$ formalism can be recovered using a fluid treatment.

\section{Tailoring of beam cross-section by excitation of transverse collective oscillations}

The presence of warm-plasma oscillations in a space-charge-dominated beam not in steady state has been established by the experimental and simulation evidence discussed above. It has also been established that some of the properties of these oscillations can be reproduced by a warm 
fluid description. However, the conditions under which the warm-fluid treatment adequately describes the evolution of the beam distribution are not firmly established. The warm-fluid equations are derived by neglecting transverse heat flow in order to truncate the moment expansion of the perturbed distribution function. It is therefore reasonable to expect that this approach will be adequate to describe the beam evolution for relatively short propagation lengths provided that the beam kinetic temperature is sufficiently low. One region of a heavy ion fusion driver where this is true may be in the final focus region as the beam converges from the final focusing lens system onto the fusion target.

Most simulations of the characteristics of the beam propagation through the final lens system onto the target have found that the beam profile at the target is peaked at the center. Recent target designs however, benefit from a hollow beam illumination for maximum efficiency [28]. The oscillatory behavior of the warm-plasma modes in the beam suggests a method for generating a degree of hollowing at the focal spot, provided the warm-fluid description of the beam is adequate over the scale necessary for focusing the beam at the chamber wall.

To examine this possibility a series of numerical experiments were performed starting with a hollow beam that has a uniform temperature and allowing the beam to expand freely. After $3 \mathrm{~m}$ of expansion, the assumed distance to the chamber wall, the average outward velocity as a function of radius, as well as the density and kinetic temperature, are measured from the simulation. Assuming the fluid description is adequate, a beam is then initialized with the same temperature and density variation but with the average velocity reversed so that the beam now coasts inward. This is the fluid moment equivalent of time-reversing the system of equations. If the fluid description accurately describes the beam system, this reversal should form a distribution at the focal spot that approximates the hollow distribution that the procedure started with.

While a comprehensive study of the range of validity of this approach has not yet been undertaken, one example was generated by assuming a spot $5 \mathrm{kA}$ of $4 \mathrm{GeV}$, singly ionized $\mathrm{Pb}$ with a radius of $3 \mathrm{~mm}$, which is allowed to expand for $3 \mathrm{~m}$. This emittance is one quarter of the emittance which would generate a contribution to the beam spreading which is equal to the spreading due to space charge over the $3 \mathrm{~m}$ length. Note that the numerical system is fully reversible so that if all particle velocities were reversed after the $3 \mathrm{~m}$ of propagation the beam distribution would come back to the original spot configuration. This exact reversibility is lost, however, when the beam is approximated by its fluid moments.

The beam profile at the spot is plotted in Fig. 1. Note that this is a plot of density along the principal axes, and such a plot substantially understates the degree to which the beam energy is deposited at the outside of the beam, so that the effective degree of hollowing is also understated.

Further insight into the detailed mechanisms involved in this simulation can be obtained by looking at the details of the fluid moments of the beam after expansion. Fig. 2 is a plot of the inward velocity, as a function of radius, which is imparted to the beam at the chamber radius. Note that the nonlinearity in not large. The plot of temperature as a function of radius in Fig. 3 however, shows a

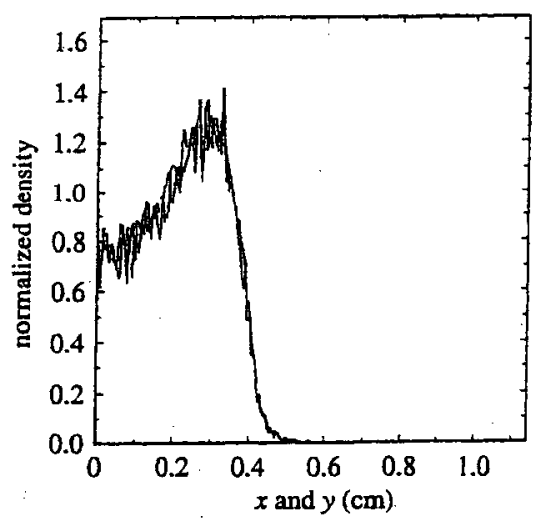

Fig. 1. Beam transverse current density measured along the principal axes for a collapsing beam at the focal spot. The density and temperature in the simulation were initialized to match values measured from an initially hollow beam allowed to expand, while the average velocity as a function of radius were initialized as the reverse of the measured value. If the fluid moment description were accurate the spot profile would be completely hollowed. 


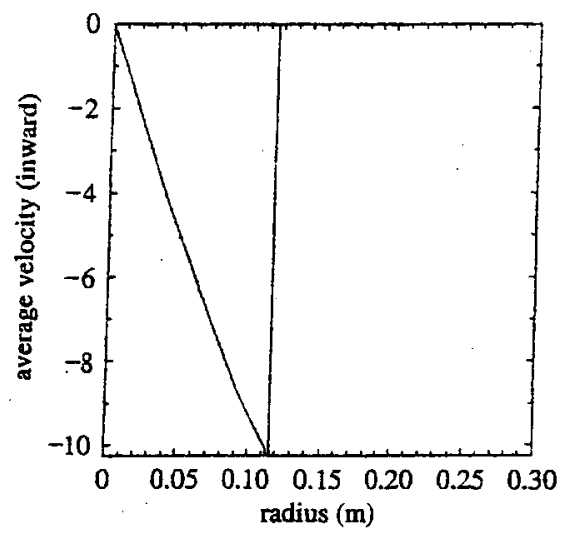

Fig. 2. A plot of the relative inward velocity, as a function of radius, which is measured from the expanding beam and imparted as an initial inward velocity to the collapsing beam.

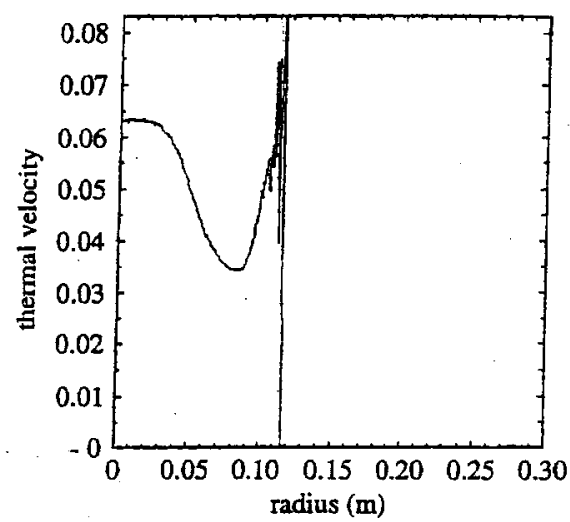

Fig. 3. Initial relative thermal velocity as a function of radius for the collapsing beam.

substantial nonuniformity. Fig. 4 which is plot of the density as a function of radius, also exhibits comparable deviation from uniformity. It is therefore evident that the hollowing observed at the spot results not from a nonlinearity in the focusing but rather from the launch of a large amplitude warm-plasma oscillation at the correct phase so that the beam exhibits a hollow profile at the focal spot.

Substantial further investigation would be required to probe the sensitivity of the spot hollowing to small changes in the initial conditions. Further investigation would also be required to determine whether relaxing the requirement for a uniform temperature at the spot, as assumed in

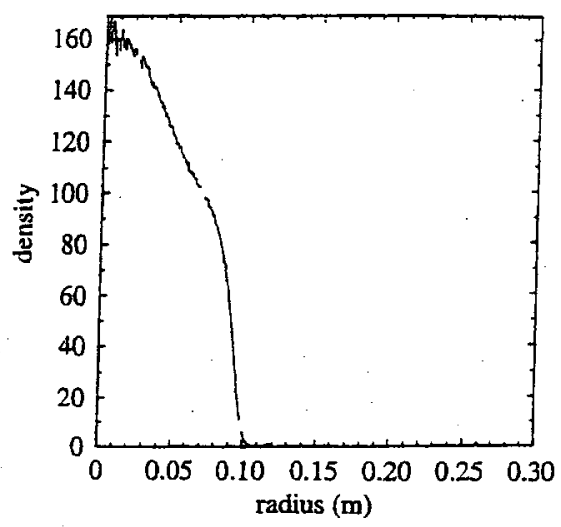

Fig. 4. Initial relative density as a function of radius for the collapsing beam. Note that the temperature and density profiles are very similar to an $n=2$ axisymmetric Gluckstern mode.

this example, would simplify achievement of a hollowed spot profile. The issue of achieving a degree of hollowing for beams of greater emittance is also in need of investigation. And the issue of how to tailor in a practical manner the distribution entering a real chamber is another significant issue among many which should be resolved before such a hollowed distribution can be assumed to be practically realizable. However, the concept of using the launch of a large amplitude plasma mode to tailor the beam behavior appears to be a fertile area for further investigation. This is particularly true if it is noted that the temperature and density distributions which were found necessary to achieve the hollow spot are very similar to the single $n=2$ axisymmetric Gluckstern eigenmode as is plotted by Lund and Davidson [26]. The excitation and propagation of such a single mode appears to be simpler than imposing a more general requirement on tailoring the beam distribution. It is also likely based, on previous observation, that such a low order single mode will retain its coherence for a relatively long propagation distance.

\section{Conclusions}

Recent theoretical, experimental and simulation evidence point to the potential universality of the excitation of warm plasma oscillations when a 
space charge dominated beam is not in detailed local equilibrium with the external focusing forces. If these modes are not driven unstable by some source of driving energy, details of the relaxation of the beam to equilibrium can then be viewed in terms of the evolution of these modes. This picture allows for a detailed understanding, in relatively simple terms, of the internal dynamics of spacecharge-dominated beams and opens the possibility that a degree of control of the beam evolution can be exercised by exploiting the normal modes of the space-charge-dominated beam system to tailor the beam.

While this picture has gained acceptance relatively recently, it has already simplified the explanation of various experimental observations and shows substantial promise for aiding in the optimal design of a variety of space-chargedominated accelerator systems.

\section{References}

[1] A. Friedman, D.P. Grote, I. Haber, Phys. Fluids B 4 (1992) 2203.

[2] A. Friedman, Beam simulations for IRE and driver status strategy, Nucl. Instr. and Meth. A 464 (2001) 653, these proceedings.

(3) P. O'Shea, M. Reiser, The University of Maryland electron ring, Nucl. Instr. and Meth. A 464 (2001) 646, these proceedings.

[4] D.P. Grote, A. Friedman, G. Craig, I. Haber, W.M. Sharp, Progress toward source-to-target simulation, Nucl. Instr. and Meth. A 464 (2001) 563, these proceedings.

[5] I.M. Kapchinskij, V.V. Vladimirskij, Limitations of proton beam current in a strong focusing linear accelerator with beam space charge, in: L. Kowarski (Ed.), Proceedings of the International Conference on High Energy Accelerators and Instrumentation, CERN, Geneva, 1959, p. 274.

[6] L. Gluckstern, Oscillation modes in two dimensional beams, Proceedings of the Linac Conference, Fermilab, 1970 , p. 811.

[7] R.C. Davidson, Physics of Nonneutral Plasmas, AddisonWesley, Redwood City, CA, 1990.

[8] I. Hofmann, L.J. Laslett, L. Smith, I. Haber, Part. Accel. 13 (1983) 145.

[9] J. Struckmeier, J. Klabunde, M. Reiser, Part. Accel. 15 (1984) 1344.
[10] T.P. Wangler, K.R. Crandall, R.S. Mills, M. Reiser, IEEE Trans. Nucl. Sci. NS-32 (1985) 2196.

[11] O.A. Anderson, Some mechanisms and time scales for emittance growth, in: M. Reiser, T. Godlove, R. Bangerter (Eds.), Heavy Ion Inertial Fusion, AIP Conference Proceedings 152, AIP, New York, 1986, p. 253.

[12] I. Haber, D. Kehne, M. Reiser, H. Rudd, Phys. Rev. A 15 (44) (1991) 5194.

[13] R.A. Jameson, IEEE Trans. Nucl. Sci. NS-28 (1981) 2408.

[14] I. Hofmann, IEEE Trans. Nucl. Sci. NS-28, 2399.

[15] I. Hofmann, Phys. Rev. E 57 (4) (1998) 4713.

[16] R.A. Kishek, S. Bernal, Y. Li, P.G. O Shea, M. Reiser, M. Venturini, W. Zhang, I. Haber, Computational studies of collective effects for heavy ion inertial fusion (HIF) beams, Nucl. Instr. and Meth. A 464 (2001), these proceedings.

[17] A. Friedman, D.A. Callahan, D.P. Grote, A.B. Langdon, I. Haber, Bull. Am. Phys. Soc. 35 (9) (1990) 2121.

[18] I. Haber, D.A. Callahan, A. Friedman, D.P. Grote, A.B. Langdon, Transverse-longitudinal temperature equilibration in a long uniform beam, Proceedings of the International Symposium on Heavy Ion Fusion, J. Fusion Eng. Design 32-33 (1996) 169.

[19] I. Haber, D.A. Callahan, A. Friedman, D.P. Grote, A.B. Langdon, Transverse-longitudinal energy equilibration in a long uniform beam, Proceedings of the 1995 Particle Accelerator Conference, p. 3283.

[20] T.F. Wang, L. Smith, Part. Accel. 12 (1982) 247.

[21] I. Haber, D.A. Callahan, A. Friedman, D.P. Grote, S.M. Lund, T.F. Wang, Nucl. Instr. and Meth. A 415 (1999) 405.

[22] S.M. Lund, D.A. Callahan, A. Friedman, D.P. Grote, I. Haber, T.F. Wang, Theory of an electrostatic instability driven by transverse-longitudinal temperature anisotropy in space charge dominated beams, Proceedings of the XIX International Linear Accelerator Conference, Chicago $\Pi$, August 23-28, 1998, in preparation.

[23] S.M. Lund, J.J. Bamard, G.D. Craig, A. Friedman, D.P. Grote, H.S. Hopkins, T.C. Sangster, W.M. Sharp, S. Eylon, T.J. Fessendon, E. Henestroza, S. Yu, I. Haber, Nucl. Instr. and Meth. A 415 (1998) 345.

[24] S. Bernal, P. Chin, R.A. Kishek, Y. Li, M. Reiser, J.G. Wang, T. 'Godlove, I. Haber, Phys. Rev. (Special Topics, Accelerators and Beams) 1 (4) (1998).

[25] S. Bernal, R.A. Kishek, M. Reiser, I. Haber, Phys. Rev. Lett. 82 (1999) 4002.

[26] S.M. Lund, R.C. Davidson, Phys. Plasmas 5 (1998) 3028.

[27] R.C. Davidson, S. Strasburg, Warm-fluid stability properties of intense nonneutral charged particle beams with pressure anisotropy, Phys. Plasmas (2000), in press.

[28] D.A. Callahan-Miller, M. Tabak, Nucl. Fusion 39 (1999) 883. 


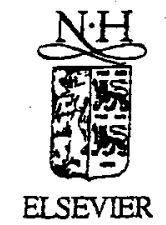

Nuclear Instruments and Methods in Physics Research A 464 (2001) 484-492

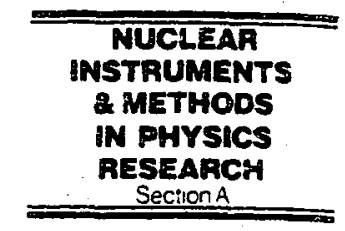

Www.elseviernllocaternima

\title{
Transverse space-charge modes in non-equilibrium beams
}

\author{
R.A. Kishek ${ }^{\mathrm{a}, *}$, S. Bernal ${ }^{\mathrm{a}}$, P.G.O Shea ${ }^{\mathrm{a}}$, M. Reiser ${ }^{\mathrm{a}}$, I. Haber ${ }^{\mathrm{b}}$ \\ anstitute for Plasma Research, University of Maryland, College Park, Bldg No. 223, MD 20742, USA \\ ${ }^{\mathrm{b}}$ Naval Research Laboratory, Washington, DC, USA
}

\begin{abstract}
Successful operation of a Heavy Ion Fusion driver requires knowledge of how the beam responds to various perturbations from equilibrium. Recently, simulations and experiments have verified predictions that space-chargedominated beams can carry waves, observable as modulations in the particle density and temperature. Self-consistent simulations using the particle-in-cell code WARP explore the evolution and scaling of space charge modes, both in linear and circular machines. Examples of phenomena where these collective modes play a critical role are provided. In circular focusing arrays, the modes resonate when driven by lattice errors, at frequencies which depend on the space charge intensity. Collective modes are shown to lead to emittance exchange and energy equipartitioning in an anisotropic system. Scaling relations are determined for some of these effects. (C) 2001 Elsevier Science B.V. All rights reserved.
\end{abstract}

PACS: $29.27 . \mathrm{Bd} ; 52.65 . \mathrm{Rr} ; 52.58 . \mathrm{Hm} ; 41.75 . \mathrm{Lx}$

Keywords: Space-charge-dominated beams; Space charge modes; Equilibrium; Simulations; Particle-in-cell; Circular accelerators; Heavy ion fusion

\section{Introduction}

Successful operation of a Heavy Ion Fusion (HIF) driver requires precise understanding of how the beam distribution evolves under the myriad operations and how it encounters from the source to the target. Examples include merging, acceleration, bending, compression and beam shaping in the final transport. Recently, a new perspective has emerged whereby a beam acts as a medium for carrying a number of density and

\footnotetext{
*Corresponding author. University of Maryland, College Park, MD 20742, Tel.: + 1-301-314-9437; fax: +1-301-4055012.

E-mail address: ramiak@ebte.umd.edu (R.A. Kishek).
}

temperature modes that interact with the surrounding structure and with each other via the space charge force [1]. Although such a wave description has been proposed in theoretical works as early as 1970 by Gluckstern [2] and subsequently by others [3-5], experimental evidence has been lagging. This collective mode structure has finally been revealed in experiments at the University of Maryland on the transport of lowenergy electron beams having the same velocity and tune depression as HIF beams [6]. The experiments demonstrated the creation of a bighar-density ring-shaped perturbation at the beam edge consequent to phase space distortion by an aperture. Further downstream, the ring travels from the beam edge to the center while additional 
rings are induced near the edge in a wavelike fashion, with an oscillation frequency dependent upon the betatron and plasma frequencies of the beam. The origin of these radial modes has been traced back to the fact that the beam emerging from the aperture is not in equilibrium, i.e., the aperture perturbs the beam. Simulations using the particle-in-cell (PIC) code WARP [7] accurately reproduce the density modes, in excellent agreement with the experiment [6]. Simulations reveal that the oscillations are damped, and the beam relaxes to an equilibrium after a few plasma periods, without rms emittance growth. As we shall see below, the temperature profile of the beam plays an equal role to the density profile in determining the dynamics of the modes.

The lack of adverse effects in Bernal's experiment may provide a false security that these collective modes may be of little practical significance and can thus be safely ignored. Yet, the experiment in [6] sampled only a narrow range of parameter space, and the beamline was only about one plasma period long. A vast arena of situations remain where the beam manipulations have interesting and significant consequences on the evolution of beam modes. The lowest-order Gluckstern surface modes, for example, have since inception been identified with the familiar envelope modes for an rms mismatched beam. Our concern in this paper is the myriad higher-order modes, which can appear even in an rms matched beam if the distribution is not matched in detail. We therefore apply self-consistent calculations with WARP to further probe the parameter space in preparation for further experiments. We restrict the discussion on the space charge modes in the plane transverse to the direction of motion. First, we examine the scaling of the wave velocities with the beam parameters. Next, we consider the possibility of lattice errors driving the modes into resonance in a circular machine. Finally, we introduce the possibility of having an energy anisotropy in the two transverse directions, as can happen for example in a dispersive system. As we shall see coupling via space charge modes leads to a process of equipartitioning. We conclude by commenting on future possibilities and on planned experiments.

\section{Simulation model, numerics, and tools}

Where applicable, we have used a lattice of the University of Maryland Electron Ring (UMER) $[8,9]$ as a model. UMER is intended to be a scaled version of an HIF recirculator [10], and the results are also applicable to the bends in a linac driver. The beam energy is kept low $(10-50 \mathrm{keV})$ to achieve the same $\beta$ as heavy ions in full-scale drivers. An intense tune depression $\left(v / v_{0}=\lambda_{\beta 0} / \lambda_{\beta}=0.13\right.$, where $\lambda_{\beta}$ is the betatron wavelength and the subscript 0 denotes zero space charge) can be achieved at the nominal beam current of $100 \mathrm{~mA}$, energy of $10 \mathrm{keV}$, and unnormalized effective $\left(4^{*} \mathrm{rms}\right)$ emittance of $50 \mathrm{~mm}$ mrad. The lattice consists of 36 FODO cells around the $11.52 \mathrm{~m}$ circumference ring. The external focusing is set to obtain a zero-current phase advance $\sigma_{0}=76^{\circ}\left(v_{0}=7.6\right)$, resulting in an average beam radius of $10.2 \mathrm{~mm}$.

Our main computational workhorse is the PIC code WARP [7], which has been developed at Lawrence Livermore National Laboratory (LLNL) for heavy ion fusion applications and can self-consistently track a space-charge-dominated beam along bends. The simulations use the $2 \mathrm{~d} 3 \mathrm{v}$ single slice version of WARP, which advances particles in a transverse slice under the action of external forces and the self-consistent self-fields, but key simulations have also been tested using the full 3-D version. The numerics have been chosen and tested to assure convergence. A $256 \times 256$ grid is used for the Poisson solver ( $\sim 50$ cells/beam radius), while a step size of $4 \mathrm{~mm}$ along $s$ is substantially smaller than any of the characteristic wavelengths of the system. Typically 20,000 particles are used combined with Gaussian filtering in the Poisson solver so as to reduce the effects of numerical collisions. Although this amounts to only 2.5 particles per cell and a reduced effective resolution, the model is reasonable. Test simulations using no filtering and $160-400,000$ particles (20-50 particles per cell) showed little difference. The numerics were tested independently for each geometry, and the resolution and statistics employed appear adequate. The aforementioned detailed agreement of the WARP simulations with Bernal's experiment [6], whose 
beam is very similar to the ones discussed here, represents a further benchmark for the validity of this work.

In the simulations presented here, we opt to start with a semi-Gaussian (S-G) distribution [uniform in space; Gaussian in velocity with uniform spread], that is matched only in the rms sense. Agreement with experimental measurements [6] demonstrates that this non-equilibrium distribution accurately represents an experimental beam immediately after its creation at the source, or after passage through an aperture sufficiently small to truncate the thermal edge of the distribution. In Bernal's experiment, the aperture is 3 times smaller than the beam upstream from it. Hence the emerging inner core very much resembles a S-G, since any existing density gradient at the edge of the beam is wiped out (although we do have evidence for a small remnant temperature gradient). After the aperture, we observe the formation of another thermal sheath at the edge by means of the space charge waves discussed herein.

We remark that existing theory [2-5] is based on the stationary Kapchinskij-Vladimirskij (K-V) distribution [a Kronecker $\delta$-function in 4-D phase space, which also projects a uniform density in $x$ and $y$ ]. Since the $K-V$ distribution essentially consists of a singularity in phase space and lacks the Maxwellian spread in particle velocities, it does not allow for natural damping mechanisms in beams. Hence many authors $[1,4,5,11]$ have suggested that a $\mathrm{K}-\mathrm{V}$ beam model, the only one amenable to analytic treatment, distorts the growth rates of modes and leads to predictions of non-physical instabilities. A detailed discussion of these issues goes beyond the scope of this paper, so we restrict simulations here to the more realistic S-G distribution.

For ease of comparison to theory it is advantageous to be able to identify the modes present in a particular simulation. According to the formulation of Gluckstern [2] for the azimuthal modes in a cylindrical beam, the potential inside the beam for each mode is of the form:

$\Phi_{n}^{l}(r, \theta)=P_{n}(r)[\cos l \theta ; \sin l \theta]$

where the $P_{n}$ is a polynomial of order $n$, the radial mode number; while $l$ is the azimuthal mode number. The cosine term occurs for modes with even symmetry, while the sine term denotes odd symmetry. Modes of this form can be easily identified in particle simulations if we Fourier transform the potential inside the beam, or alternatively the particle density over the azimuthal angle, $\theta$. After scaling the resultant coefficients by the integral of $P_{n}$, we obtain the strength of each of the odd and even azimuthal modes.

\section{Scalings of isotropic space charge waves}

The first task we assumed was to widen the parameter space explored from the narrow range sampled experimentally in Ref. [6]. Once UMER is online we will have access to experimental data over a wide range of tune depressions, but at the present time are restricted to simulation studies. Of particular interest is the variation of some characteristic parameter, say the periodicity or the damping time, as a function of a dimensionless parameter characterizing the space charge, such as the tune depression, $k / k_{0}$. To simplify the exercise, we choose the external focusing to be uniform along $z$ (and equal in $x$ and $y$ ), resembling the focusing obtained from a uniform distribution of background ions. A $4 \mathrm{kV}, 17 \mathrm{~mA}$ electron beam with an emittance of $67 \mathrm{~mm}$-mrad is launched with a beam size rms matched to the channel. A S-G distribution models the aperture, as discussed above. The tune depression is varied by adjusting the external focusing in each simulation.

The observed oscillations in the beam density are actually a superposition of a number of Gluckstem-like modes [5], each having its own frequency according to its own branch of the dispersion relation. Without decomposing the distribution into individual modes, it is difficult to talk about an oscillation frequency. Rather we examine the mode group velocity, which we define as the speed by which the perturbation moves from the edge to the center (measured from the similations), and display in Fig. 1 as a function of tune depression. For normalization, we express it in terms of the zero-current betatron period, $\lambda_{\beta 0}$, in the same focusing channel divided by the 


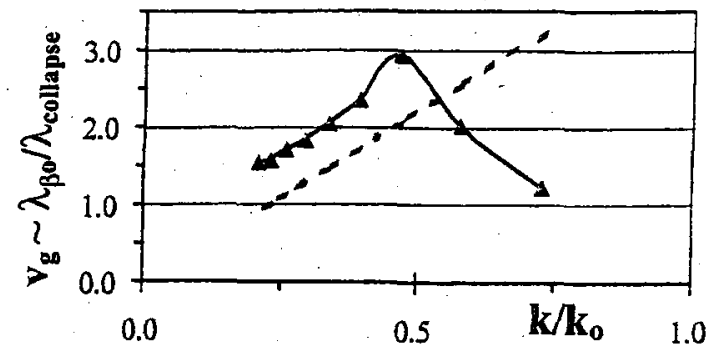

Fig. 1. The ratio of the zero-current betatron period to the propagation distance over which the perturbation collapses, as a function of tune depression. (The discreteness of the sampling results in some error bars). The dotted line reflects the transverse thermal velocity from the emittance term.

propagation distance over which the perturbation collapses. In the limit of zero-space charge, the perturbation collapses in about one zero-current betatron period, while in the limit of zero temperature, the perturbation collapses in about one plasma period, $\lambda_{\mathrm{p}}=\lambda_{\beta 0} / \sqrt{2}$. In between, the perturbation evolves on a faster time scale than either betatron or plasma oscillations, peaking at a tune depression near 0.5. The dotted line in Fig. 1 represents the rms transverse thermal velocity for each simulation, normalized to the same parameters. Note that in the left half of the figure (high space charge), the group velocity increases almost linearly with thermal velocity. The right half, on the other hand, suggests that the different modes contained in the localized perturbation widely disperse. Thus we can define the space-chargedominated regime as that region where wave behavior is predominant.

\section{Resonances in circular machines}

Space charge modes can become particularly detrimental when they resonantly interact with the lattice superperiodicity in circular machines $[12,13]$. Thus far, most storage rings have been operated with a very low space charge tune shift, rendering the exact description of resonance using the modes formulation superfluous. However, the demand for higher current, such as in HIF drivers, introduces the need for the detailed understanding of resonance mechanisms. This interaction is clearly illustrated in the following simulations [14]. The quadrupoles in the UMER lattice were given a random set of higher-order harmonic errors in accordance with measurements on the actual magnets using a rotating coil [15]. Fig. 2 shows the evolution of the effective beam emittance $\left(4^{*} \mathrm{rms}\right)$ for various operating points. The control simulation which uses ideal magnets shows a small growth in emittance ( $\sim 10 \%$ over 10 turns or $.115 \mathrm{~m}$ ) due to numerical collisions (Fig. 2 (lowest curve)). ${ }^{1}$. The addition of systematic harmonic errors (i.e., all magnets are identical) does not necessarily degrade the transport, and the same curve results. The addition of random harmonic errors, however, shows a rapid $(5-10$ turns) deterioration of beam quality (for the nominal machine tune and beam current). The deterioration is seen as a rapid emittance growth accompanied by halo formation (Fig. 2 (upper curve)).

The remaining two lines in Fig. 2 correspond to cases where the emittance growth is reduced by slightly shifting the beam current and/or tune. This indicates the presence of a resonance near the original operating point in the simulations. We can move away from that resonance in two ways: (1) adjusting the external focusing, consequently changing both the depressed and undepressed tunes; and (2) adjusting the beam current, thus only changing the depressed tune. In this example, the beam current was reduced from 100 to $98 \mathrm{~mA}$ then to $96 \mathrm{~mA} .^{2}$ The undepressed tune was kept at $v_{0 x}=7.6$ and $v_{0 y}=7.73$; only the depressed tune was changed from $v_{x}=1.02$ to 1.53 then 1.90 , and from $v_{y}=1.06$ to 1.58 and 1.97 , respectively. Given the undepressed tune of $\sim 7.6$, singleparticle theory predicts a decapole resonance. A simple substitution of $v$ for $v_{0}$ predicts a dipole resonance for the original operating point. Both predictions contradict the observation: by applying the modal decomposition technique described above, we detect the growth of the odd sextupole

\footnotetext{
${ }^{1}$ This growth can be made arbitrarily smaller by increasing the number of particles and scales as $1 / \sqrt{n_{p}}$.

${ }^{2}$ The emittance was adjusted in the simulation in order to keep the beam size the same, so that the beam may sample the same strengths of the higher-order harmonics.
} 


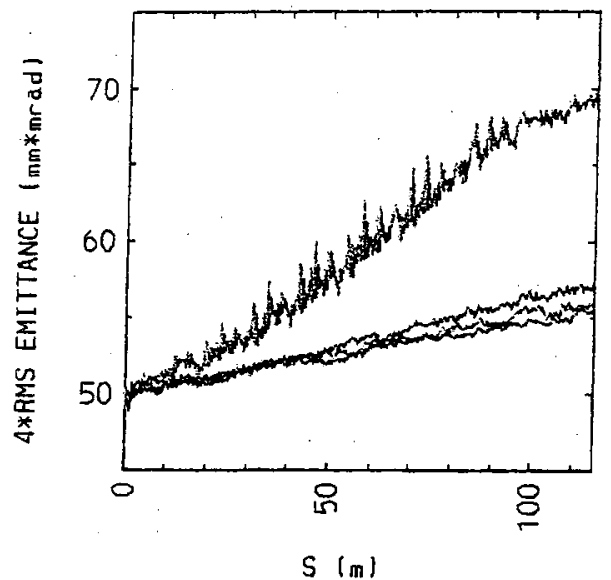

Fig. 2. Emittance growth over the first 10 turns due to resonances from random harmonic errors in the quadrupoles. The upper line is for a beam current of $100 \mathrm{~mA}$ and a normalized effective emittance of $50 \mathrm{~mm}$-mrad. The bottom three lines show cases where the resonance is avoided either by making all magnets identical or by shifting the tune to $98 \mathrm{~mA}$. and $75 \mathrm{~mm}$-mrad, then to $96 \mathrm{~mA}$ and $94 \mathrm{~mm}$-mrad. The residual slow emittance growth is due to numerical collisions and can be shown to disappear upon increasing the number of simulation particles.

mode. The excitement of an odd sextupole resonance for these parameters is in agreement with a recent theory based on the $\mathrm{K}-\mathrm{V}$ distribution by Venturini and Gluckstern [13]. Simulations using $\mathrm{K}-\mathrm{V}$ distributions are qualitatively similar to the ones above with a semi-Gaussian. The odd sextupole mode resonates in both cases at the same operating point, accompanied by emittance growth and halo formation. The growth rates of the mode, however, differ by a large factor between the two distributions, corroborating the suspicion about the applicability of a $\mathrm{K}-\mathrm{V}$ distribution mentioned above.

\section{Energy anisotropy, equipartitioning, and dispersion}

So far, the discussion has centered on isotropic beams, meaning that the beam temperature in the horizontal plane $(x)$ was equal to that in the vertical plane (y). Nevertheless, in many accelerators, the beam may be subject to anisotropy, as

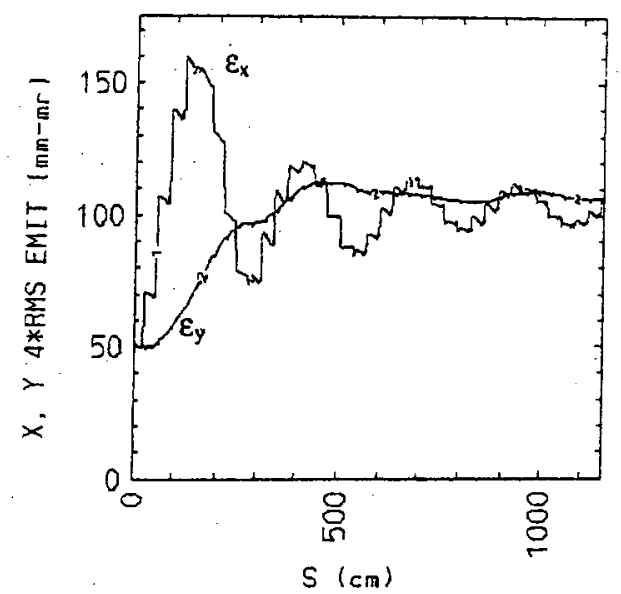

Fig. 3. Emittance growth due to a dispersion mismatch when a. beam with $\Delta p / p=0.015$ enters a ring from a straight injector. Dispersion theory predicts no change in $\varepsilon_{y}$, but emittance exchange due to equipartitioning is observed. The final emittances are roughly equal since the external focusing is symmetric in $x$ and $y$.

is possible, for example, in the injector bends or near the final focus. Injection into a dispersive lattice leads to an apparent increase in emittance in the bend plane, but not in the other transverse plane. Simulations of dispersion, however, have revealed a rapid exchange between the two emittances and a resulting equilibration over 1 turn with equal transverse temperatures [16-18], as illustrated in Fig. 3. Another scenario is the transverse-longitudinal equipartitioning $[11,19,20]$. Acceleration leads to cooling in the longitudinal velocity spread, while compression causes the reverse to be true. In general, both result in a severe anisotropy between the longitudinal and transverse planes. An understanding of energy transfer via space charge waves in the transverse directions is likely to shed light on transverselongitudinal coupling, an important factor to be considered in designing acceleration and compression schedules.

This section extends the wave analysis to the case where the temperatures are different. In the presence of non-linear coupling mechanisms, the oram can be predicted to equipartition, or reach a state where the velocity spreads in all three directions are equal. The final equilibrium 
situation can be predicted using thermodynamic considerations [11]. In space-charge-dominated beams, Coulomb collisions are infrequent to account for the energy transfer, whereas space charge waves have been shown to be a possible coupling candidate [20-22]. Unfortunately, there has been very little experimental data on this equipartitioning process. Despite the pioneering effort of Hofmann and others in the early 1980 s (see, e.g. [21]), simulation studies with realistic distributions have likewise been sparse. The parameter space in this case has been thought to be too large to bridge, and many of the early simulations were restricted to unrealistic distributions such as the $\mathrm{K}-\mathrm{V}$ and the waterbag. Since existing theory [23] is also based on the $\mathrm{K}-\mathrm{V}$ distribution, it is subject to the pitfalls discussed above.

We therefore explore the role of space charge modes in coupling and equipartitioning using the non-equilibrium S-G distribution in WARP. The $\mathrm{K}-\mathrm{V}$ distribution gives substantially different results, which are discussed in Ref. [24]. The UMER model is used as above, except that the unnormalized effective emittance is chosen to be initially different in $x$ and $y, \varepsilon_{x}=100 \mathrm{~mm}$-mrad, while $\varepsilon_{y}=50 \mathrm{~mm}$-mrad. This means that the tune depressions are different, namely $\left(v / v_{0}\right)_{x}=\lambda_{\beta 0} / \lambda_{\beta x}$ $=0.25 ;\left(v / v_{0}\right)_{y}=\lambda_{\beta 0} / \lambda_{\beta y}=0.13$. The initial beam sizes in $x$ and $y$ are chosen to be matched solutions of the rms envelope equations, assuming the emittances will not change. Naturally, any change in emittance will also induce an rms mismatch. Fig. 4 shows the evolution of the beam emittance in $x$ and $y$ for a FODO lattice in a straight channel. We have found that the details of the focusing have little effect on the outcome: a uniform focusing channel gives the same result as a FODO channel, while the addition of bends does not change the emittance picture much. In all cases the emittances smoothly change as the beam redistributes and reach a final state where the beam velocity spread is equal in the two transverse directions. If we take into account the details of the initial distribution, the final emittance is consistent with conservation of energy as predicted from a thermodynamic model $[11,17]$.

Close examination of the density profile of the beam (Fig. 5) reveals that the energy transfer

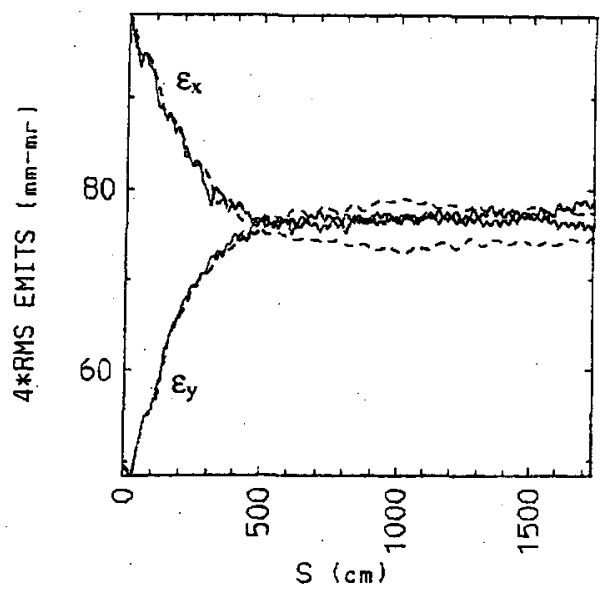

Fig. 4. Equilibration and emittance exchange for a beam propagating in a FODO channel with no bends and starting with $\varepsilon_{x}=2 \varepsilon_{y}$ (solid). The dotted curve represents a uniform channel with the same tune.

mechanism is precisely the coupling induced by the space charge modes which we have seen in the symmetric case [6]. Recalling that the speed of propagation of the density crests from the edge to the center depends on the tune depression, the wave speed in an anisotropic beam depends on the direction, since the tune depression is initially different in $x$ and $y$. In Fig. 5, what begins as a ring at the beam edge transforms into an ellipse, and subsequently higher-order modes, breaking the symmetry and naturally coupling the two transverse directions. In other words, an initial temperature anisotropy translates into a density anisotropy downstream. Kinetic energy is thus transferred from one plane to the other, leading to an equipartitioned beam in a distance of about $5 \mathrm{~m}$, roughly equivalent to 2.5 plasma periods. This is precisely the same mechanism observed in Fig. 3 after dispersion induces the anisotropy.

Of practical interest is the rate at which equipartitioning takes place (defined here as defined as the inverse of the distance over which the emittances intersect for the first time normalized to $\lambda_{\beta 0}$ ), and the scaling of that rate against various parameters of the system. In Fig. 6 the bear current is systematically varied to explore the dependence on space charge tune depression. Notice that the rate of equipartitioning follows the 


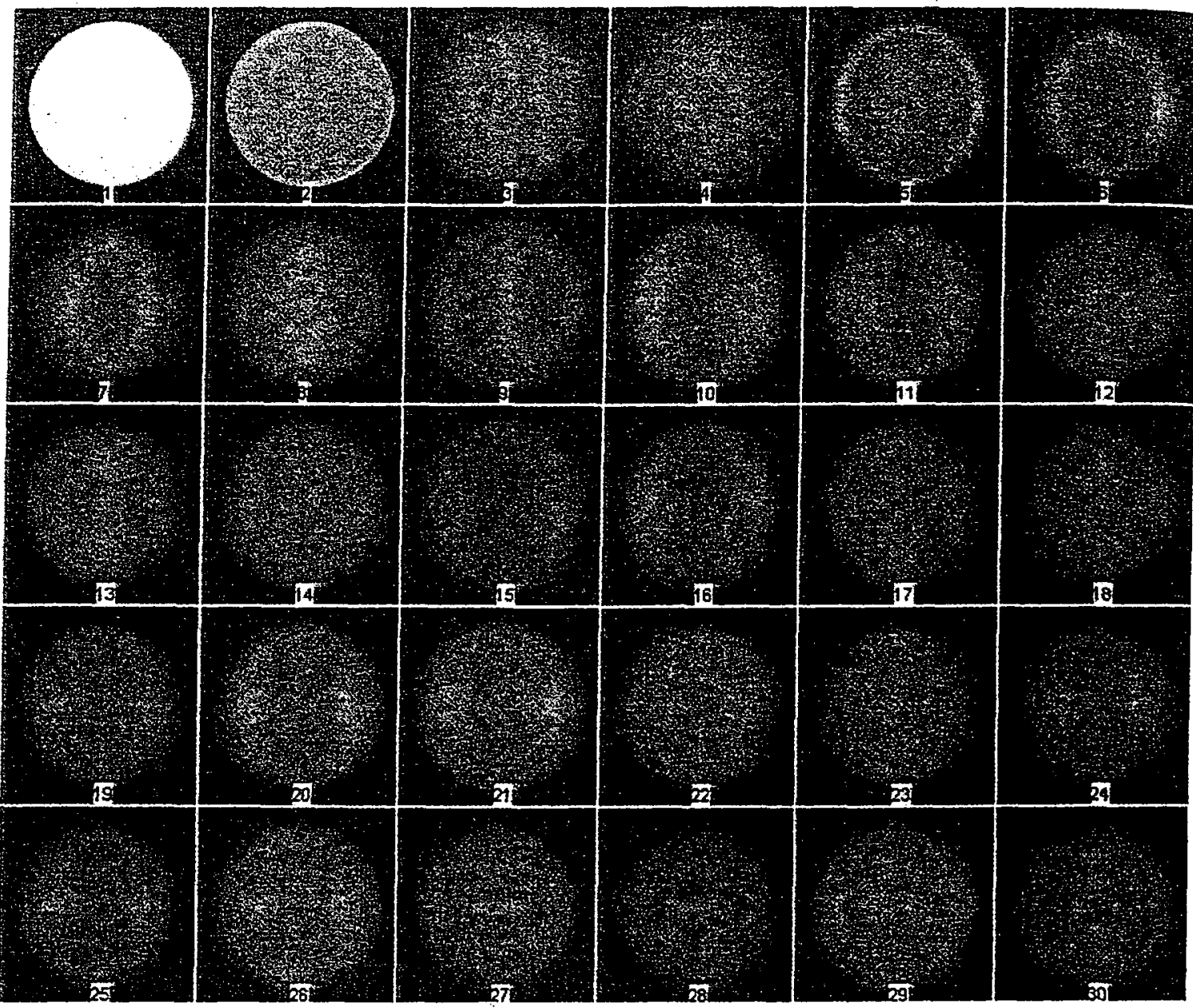

Fig. 5. Intensity diagrams of beam density for the uniform channel case of Fig. 4 . Photos $1-26$ correspond to locations every $20 \mathrm{~cm}$ from $s=0$ to $5 \mathrm{~m}$. Photos $27-29$ are from $s=10$ to $10.4 \mathrm{~m}$, and the last photo is at $17.3 \mathrm{~m}$.

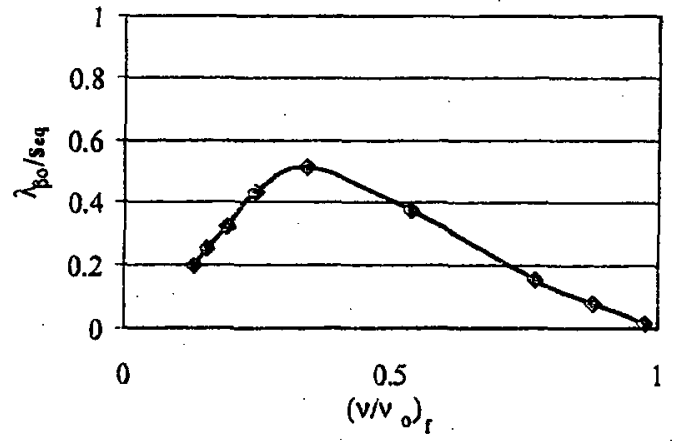

Fig. 6. Scaling of the rate of equipartitioning, defined as zerocurrent betatron wavelength divided by the distance it takes for emittances to reach an equal value for the first time, against the tune depression of the final equipartitioned beam. same bell-shaped curve in Fig. 1, indicating that it is related to the speed of propagation of the space charge modes. An additional factor here is the strength of the coupling, which depends both on the space charge content and on the strength of the initial perturbation. Since, in the limit of zerotemperature, an equilibrium thermal distribution converges to the uniform density profile of a semiGaussian, the amplitude of the initial perturbation is reduced for higher space charge. This tradeoff results in the strongest coupling taking place at some intermediate tune depression-in this case near 0.3 .

Incidentally, Ref. [16] seems to indicate that the rate of equipartitioning in a dispersive system 
depends on the energy spread of the beam. A careful comparison with the results presented therein reveal that this observed dependence is rather indirect. A beam entering a dispersive channel with a larger energy spread contains an additional portion of associated potential energy which will lead to larger emittance growth as the beam redistributes. The increase in emittance can be precisely calculated from energy conservation using the equations in Ref. [17]. Since the resulting beam has a higher thermal energy relative to the potential energy, the final tune depression will be different. If we account for this increase in thermal energy due to dispersion we find that all the simulations presented in Ref. [16] fall on the curve of Fig. 6. In other words, the processes of dispersion and equipartitioning are separable and mutually exclusive.

\section{Concluding remarks}

In conclusion, we comment on the contribution of this mode picture. It has long been suggested that space charge nonlinearities resulting from non-uniform beam distributions is at the heart of equilibration mechanisms. What we have shown in this paper-is that such a statement can only be partially true. Equilibration is a dynamic process-the initial SG beam is uniform and actually has linear space charge forces, yet nevertheless evolves into a multitude of structures. As Figs. 1, 5 , and 6 make clear, the temperature of the beam plays a major role in this dynamic evolution. We can see that the rates and the detailed evolution of such mechanisms as energy transfer depend widely on the tune depression, and not only on the beam profile. The beam temperature profile further plays a role in its evolution analogous to that of the density profile. We believe that a mode description provides a more balanced description of beam behavior, especially in the interesting intermediate regime where space charge and emittance forces are comparable.

In this paper we discussed the role that space charge modes play in a number of important phenomena: circular resonances; equipartitioning, and dispersion. This brief discussion is intended primarily to illustrate the significance of these modes and can be regarded as merely scratching the surface of this exciting topic. Clearly if we desire to build a successful large-scale driver it is imperative to better understand this phenomena and how it applies to laboratory beams. On the theoretical level, we need a better model than the singular $\mathrm{K}-\mathrm{V}$ distribution with which to formulate the wave description. Since equilibrium is often not possible to achieve in practice, it is also important to develop an understanding of how non-equilibrium beams evolve. Experimentally, the paucity of data on this wave behavior is evident. There is a clear need at this point for a well-designed experiment to systematically study space charge modes. At Maryland, UMER will offer such an opportunity. Finally, we need to improve the mode decomposition techniques so as to extract the maximum of information from experimental measurements, and to allow quantitative comparisons between theory, simulation, and experiment.

\section{Acknowledgements}

The WARP code is provided to us by the Heavy Ion Fusion group at LLNL, courtesy of Alex Friedman and Dave Grote. Many thanks to Marco Venturini for useful discussions and to Steve Lund for suggesting the mode decomposition. Finally we thank our colleagues in the UMER team, especially Matt Holland and Angie Hsieh for their assistance with running and processing some simulations.

This work is supported by the U.S. Department of Energy grant numbers DE-FG02-94ER40855 and DE-FG02-92ER54178. The WARP code runs on DOE supercomputers provided by the National Energy Research Supercomputing Center (NERSC) at LBNL.

\section{References}

[1] I. Taber, A. Friedman, D.P. Grote, S.M. Lund, R.A. Kishek, Phys. Plasmas 6 (1999) 2254, see also these proceedings. 
[2] R.L. Gluckstern, Proceedings of the National Accelerator Laboratory Linear Accelerator Conference, Batavia, $\Pi$, September 1970, p. 811.

[3] T.S.F. Wang, L. Smith, IEEE Trans. Nucl. Sci. NS-28 (3) (1981) 2477.

[4] I. Hofmann, L.J. Lasiett, L. Smith, I. Haber, Part. Accel. 13 (1983) 145-178.

[5] S.M. Lund, R.C. Davidson, Phys. Plasmas 5 (8) (1998) 3028.

[6] S. Bernal, R.A. Kishek, M. Reiser, I. Haber, Phys. Rev. Lett. 82 (May 17, 1999) 4002.

[7] D.P. Grote et. al., Fusion Eng. Des. 32-33 (1996) 193-200; also papers by Grote and Friedman in these proceedings (Nucl. Instr. and Meth. A 464 (2001) 563, 653).

[8] M. Reiser et. al., Proceedings of the IEEE 1999 Particle Accelerator Conference, New York City, NY, 1999, p. 234.

[9] P.G. O'Shea et al., The University of Maryland Electron ring (UMER), Nucl. Instr. and Meth. A 464 (2001) 646, these proceedings.

[10] T.F. Godlove, Heavy ion recirculating induction linac studies, Part. Accel. 37-38 (1992) 439-451.

[11] M. Reiser, Theory and Design of Charged Particle Beams, Wiley Inc., New York, 1994.

[12] R. Baartman, Proceedings of the Shelter Island Workshop on High Intensity Hadron Rings, Vol. 448. AIP Press, New York, 1998, p. 56.

[13] M. Venturini, R. Gluckstern, Phys. Rev. ST-AB 3 (2000) 034203.

[14] R.A. Kishek, M. Reiser, P.O. Shea, M. Venturini, W.W. Zhang, Proceedings of the Workshop on Physics of High
Brightness Beams, UCLA, November 1999. (2000), to appear.

[15] W.W. Zhang et al., PRST-AB 3, 122401 (2000).

[16] S.M. Lund, J.J. Bamard, G.D. Craig et al., Nucl. Instr. Meth. A 415 (1998) 345.

[17] J.J. Barnard, G.D. Craig, A. Friedman et al., Proceedings of the Shelter Island Workshop on High Intensity Hadron Ring, Vol. 448. AIP Press, New York, 1998, p. 221.

[18] M. Venturini, R.A. Kishek, M. Reiser, Proceedings of the Sheiter Island Workshop on High Intensity Hadron Ring, Vol. 448. AIP Press, New York, 1998, p. 278.;

R.A. Kishek, I. Haber, M. Venturini, M. Reiser, Proceedings of the Shelter Island Workshop on High Intensity Hadron Ring, Vol. 448. AIP Press, New York, 1998, p. 371.

[19] A. Friedman, D.P. Grote, D.A. Callahan, A.B. Langdon, I. Haber, Part. Accel. 37-38 (1992) 131.

[20] 1. Haber, D.A. Callahan, A. Friedman et al., Fusion Eng. Des. 32-33 (1996) 169-174; Nucl. Instr. Meth. A 415 (1998) 405.

[21] I. Hofmann, Proceedings of the Heavy Ion Fusion Workshop, Berkeley, November 1979; LBL-Report 10301, 1980; IEEE Trans. Nucl. Sci. NS-28 (1981) 2399; In: Septier (Ed.), Advances in Electronics and Electron Physics, Supplement 13C, Academic Press, New York, 1983 , pp. $49-140$.

[22] R.A. Jameson, IEEE Trans. Nucl. Sci. NS-28 (1981) 2408.

[23] I. Hofmann, Phys. Rev. E 57 (4) (1998) 4713.

[24] R. Kishek, P.G. O'Shea, M. Reiser, Phys. Rev. Lett. 85 (21) (2000) 4514. 


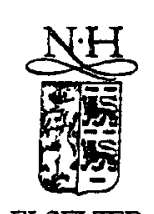

ELSEVIER

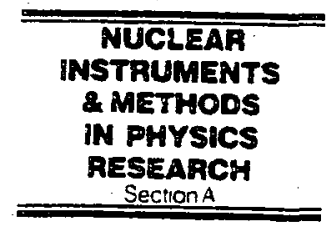

www.elsevier.ri/locate/nima

\title{
The University Maryland Electron Ring (UMER)
}

\author{
P.G. O'Shea ${ }^{a}, *$ M. Reiser ${ }^{a}$, R.A. Kishek ${ }^{a}$, S. Bernal ${ }^{a}, H^{2}$. Li $^{a}$, \\ M. Pruessner ${ }^{a}$, V. Yun ${ }^{a}$, Y. Cui ${ }^{a}$, W. Zhang ${ }^{a}$, Y. Zou ${ }^{a}$, T. Godlove ${ }^{b}$, D. Kehne ${ }^{b}$, \\ P. Haldemann ${ }^{\mathrm{b}}$, I. Haber ${ }^{\mathrm{c}}$ \\ 'Department of Electrical and Computer Engineering, Institute for Plasma Research, University of Maryland, College Park, \\ MD 20742, USA \\ ${ }^{b}$ FM Technologies, Inc. Fairfax, USA \\ ${ }^{\circ}$ Naval Research Laboratory, Washington, DC, USA
}

\begin{abstract}
A detailed understanding of the physics of space-charge dominated beams is vital in the design of heavy ion inertial fusion (HIF) drivers. In that regard, low-energy, high-intensity electron beams provide an excellent model system. The University of Maryland Electron Ring (UMER), currently under construction, has been designed to study the physics of space-charge dominated beams with extreme intensity in a strong focusing lattice with dispersion. At $10 \mathrm{keV}$, $100 \mathrm{~mA}$, the UMER beam has a generalized perveance in the range of 0.0015 , corresponding to that of proposed HIF drivers. Though compact ( $11 \mathrm{~m}$ in circumference), UMER is a very complex device. In this paper, the unique design features of this research facility, the beam physics to be investigated, and recent experimental results in the prototype injector as well as simulation studies will be reviewed. (C) 2001 Elsevier Science B.V. All rights reserved.
\end{abstract}

PACS: $29.20 .-\mathrm{c} ; 41.85 .-\mathrm{p} ; 52.58 . \mathrm{Hm} ; 52.65 . \mathrm{Cc}$

Keywords: Space-charge-dominated beams; Circular accelerators; Heavy ion inertial fusion

\section{Introduction}

Heavy ion inertial fusion (HIF) requires very intense beams with short pulses in order for the fusion target to implode and burn effectively. Proposals for HIF drivers include the possibility of recirculating induction accelerators [1]. Since such a machine would be quite a complex undertaking, risk reduction experiments are necessary, which are both inexpensive and that provide useful testbeds for design code development and bench-

${ }^{*}$ Corresponding author. Tel.: + 1-301-405-4977.

E-mail address: poshea@eng.umd.edu (P.G. O’Shea). marking. Furthermore, heavy-ion beams of the required intensity can be modeled with low-energy electron beams thus providing an excellent opportunity to study drive physics and to benchmark design codes.

Generally, recirculators and rings have been limited to lower intensities than linear accelerators. One of the most important limitations in beam current, luminosity and brightness in circular machines is the Laslett or space-charge tune shift [2]. Fear of destructive resonances has constrained existing rings to low intensity and relatively modesi tune depression. With space charge, the resonances are no longer caused by the interaction 
between the single particle orbits and the harmonics of the field errors. Instead, a resonance can occur when the frequency of a collective beam mode coincides with one of the harmonics of the error frequency spectrum.

Conventional recirculators (i.e. synchrotrons) have been limited in intensity by the necessity for the beam to back a very large number of orbits. Previous work [3] has shown that it may be possible to exceed the Laslett limit in a machine with a small number, perhaps less than 100 turns. This regime of operation is likely to be the one of choice for HIF drivers.

There has never been an opportunity to perform experiments on such recirculating machines in the region of deep tune depression, and extreme beam intensity. Therefore, almost all of our understanding in this region is based on theory, simulation and conjecture. The University of Maryland Electron Ring (UMER) [4], currently under construction, is a low-cost flexible electron model of intense ion recirculators.

In order to put UMER in a proper context, it is important to define what we mean by intensity, and to illustrate how the increased intensity influences the collective beam motion. We use the dimensionless intensity parameter, $\chi$, as the ratio of the space-charge force to the external focusing force at the beam radius $\chi=K / k_{0}^{2} a^{2}$. The space charge term is represented by $K / a^{2}$ with $K$ $=2 \mathrm{I} / I_{0}(\beta \gamma)^{3}$ is the generalized perveance, $a$ is the $2 \times$ rms beam radius, $I$ is the beam current, and $I_{0} \approx m c^{2} / 30 q\left(I_{0}=3.1 \times 10^{7}(A / Z)\right.$ A for ions with charge state $Z$ and atomic number $A$, and $I_{0}=17 \mathrm{kA}$ for electrons). The external focusing forces are represented by $k_{0}$, the zero-current betatron wavenumber. These quantities are related to the $4 \times$ rms emittance, $\varepsilon$, through the matched beam envelope equation, in the smooth approximation: $k_{0}^{2}-K / a^{2}=\varepsilon^{2} / a^{4}=k^{2}$ (where $k$ is the depressed betatron wavenumber) or $1-\chi=\varepsilon^{2} / k_{0}^{2} a^{4}=k^{2} / k_{0}^{2}$. The tune depression can then be expressed in terms of $\chi$ as $v / v_{0}=k / k_{0}=\sqrt{1-\chi}$, and the plasma wavenumber $k_{\mathrm{p}}$ as $k_{\mathrm{p}} / k_{0}=\sqrt{2 \chi}$. For a zero curre-nt, fully emittance dominated beam we have $\chi=0$, while $\chi=1$ for a fully space charge dominated beam, with zero emittance. At the lowest values of $\chi$, the motion is dominated by single particle effects and emittance. As $\chi$ approaches unity, collective plasmas oscillations become increasingly dominant. For $\chi=0.5$, the space-charge and emittance terms in the envelope equation are equal, Thus, for the range $0<\chi<0.5$, we can say that the beam radius (hence the beam physics) is emittance-dominated, while for $0.5<\chi<1$ the beam radius (physics) is space-charge dominated.

HIF drivers will likely operate with $0.89<\chi<0.98$. We see that it is possible to achieve such values of $\chi$ with electrons at $10 \mathrm{keV}, 100 \mathrm{~mA}$, $\varepsilon=50 \mu \mathrm{m}$ and $a=1 \mathrm{~cm}$. Fig. 1 shows the range of the intensity parameter for UMER. With $\chi$ ranging from 0.2 to 0.99 UMER offers a unique opportunity to study intense beam physics in a completely new regime that begins near the upper intensity range of existing machines. In the space-charge dominated regime of UMER, not only will the tune depression be unprecedented, but also the plasma frequency will exceed the zerocurrent betatron frequency. This implies that, at the high end of the UMER intensity range, collective effects will have an enormous impact on the beam dynamics. Further, the experimental and theoretical study of beam dynamics in UMER will have important applications to high-current circular accelerators other than heavy-ion fusion recirculators; examples of such applications include high-energy physics booster synchrotrons, muon colliders, and spallation neutron sources.

The unknown territory in the extreme spacecharge dominated regime will be very challenging and should provide a wealth of new phenomena. The UMER facility will allow us to investigate emittance growth due to conversion of free energy, halo formation, and equipartitioning in a circular machine. So far, these effects have only been studied in linear transport lines. In addition, UMER will permit experimental investigations of longitudinal-transverse coupling and beam profile changes resulting from dispersion; the behavior of bunch ends, resonance traversal; the longitudinal resistive wall instability; and other effects in the space-charge dominated regime that is currently inaccessible. 


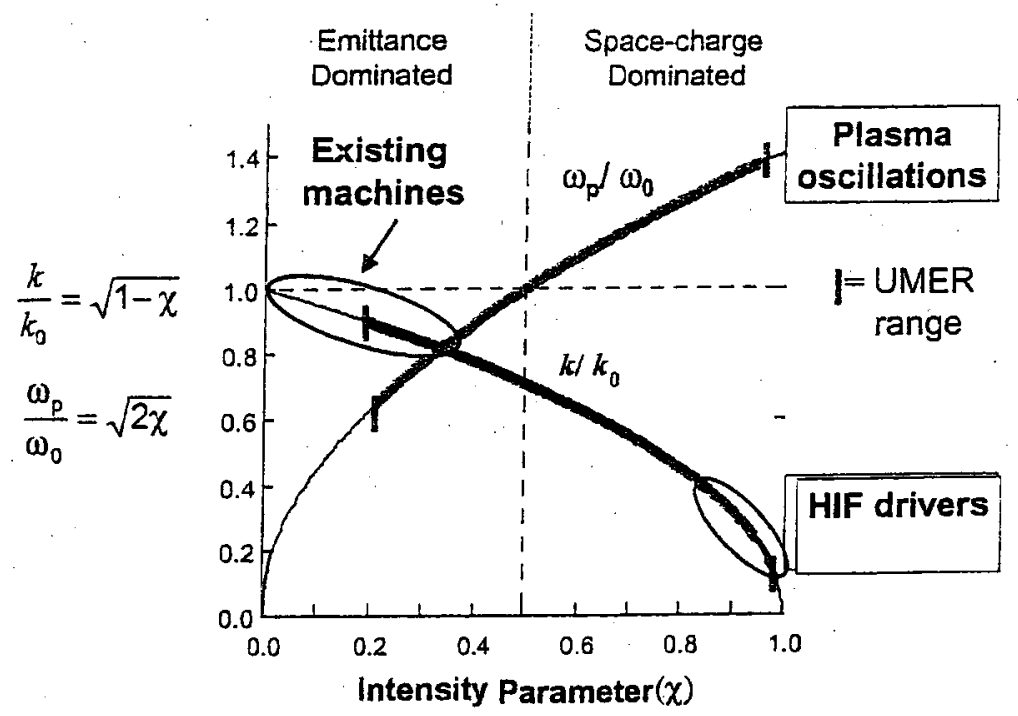

Fig. 1. Emittance dominated and space-charge dominated regimes, showing the betatron tune depression and plasma tune enhancement with increasing intensity parameter.

\section{UMER design features}

In its initial phase UMER will operate at a fixed energy of $10 \mathrm{keV}(\beta=0.2)$. Future upgrades will allow UMER to operate as a fast cycling recirculating accelerator at energies of up to $50 \mathrm{keV}(\beta=0.4)$. Table 1 gives the nominal specifications of UMER at $10 \mathrm{keV}$. The intensity parameter, tune depression or beam current can be varied in UMER over a wide range by changing to different apertures sizes in the beam collimator at the exit of the gun, by changing the anode cathode spacing, or by changing the beam energy. Several examples are shown in Table 2 for both 10 and $50 \mathrm{keV}$ operation.

A complete description of the UMER design features is beyond the scope of this paper. The reader is referred to our web page for additional details [5].

A schematic layout of UMER is shown in Fig. 2. The focusing lattice consists of 36 FODO periods of length $0.32 \mathrm{~m}$ and the ring circumference is $11.52 \mathrm{~m}$. Each FODO section contains two printed-circuit quadrupole magnets and one printed-circuit dipole. A detailed mechanical layout of a full lattice period is shown in Fig. 3. The
Table 1

UMER design specifications

\begin{tabular}{ll}
\hline Energy & $10 \mathrm{keV}$ \\
$\beta(=v / c)$ & 0.2 \\
Current & $100 \mathrm{~mA}$ \\
Generaized perveance & $1.5 \times 10^{-3}$ \\
Emittance, $4 \times$ rms, norm & $10 \mu \mathrm{m}$ \\
Pulse length & $40 \mathrm{~ns}$ \\
Circumference & $11.52 \mathrm{~m}$ \\
Lap time & $197 \mathrm{~ns}$ \\
Rep. rate & $60 \mathrm{~Hz}$ \\
Mean beam radius & $<1 \mathrm{~cm}$ \\
FODO period & $0.32 \mathrm{~m}$ \\
Phase advance, $\sigma_{0}$ & $76^{\circ}$ \\
Betatron tune, $\nu_{0}$ & 7.6 \\
Tune depression & $>0.12$ \\
\hline
\end{tabular}

zero-current phase advance per period is $\sigma_{0}=76^{\circ}$, corresponding to a tune of $v_{0}=7.6$. The maximum tune depression due to space-charge is expected to be between 0.12 and 0.2 . There are 13 diagnostic ports, and three induction modules that provide fast-rising "ear fields" to prevent expansion of the bunch ends [6] and permit acceleration to $50 \mathrm{keV}$ in a future extension of the ring operation. The electron bunch is injected into the ring at a 
Table 2

Some examples of the range of UMER parameters at 10 and $50 \mathrm{keV}$

\begin{tabular}{|c|c|c|c|c|c|c|c|}
\hline$I(\mathrm{~mA})$ & $\beta \gamma$ & $\varepsilon_{f}(\mathrm{~mm}-\mathrm{mr})$ & $\sigma_{\mathrm{or}}(\mathrm{deg})$ & $a(m m)$ & $\chi$ & $v / v_{0}$ & Assumption \\
\hline 100 & 0.20 & 50.0 & . 72.0 & 9.99 & 0.98 & 0.13 & Different tune \\
\hline 100 & 0.20 & 50.0 & 76.0 & 9.48 & 0.98 & 0.13 & Full current \\
\hline 14 & 0.20 & 18.7 & 76.0 & 3.72 & 0.89 & 0.33 & $14 \%$ aperture \\
\hline 1 & 0.20 & 5.0 & 76.0 & 1.31 & 0.51 & 0.70 & $1 \%$ aperture \\
\hline 14 & 0.20 & 37.4 & 76.0 & 4.14 & 0.72 & 0.53 & 2* emittance \\
\hline 100 & 0.20 & 250.0 & 76.0 & 10.90 & 0.74 & 0.51 & $5^{*}$ emittance \\
\hline 100 & 0.45 & 21.9 & 50.3 & 4.58 & 0.86 & 0.38 & Fuil current \\
\hline 14 & 0.45 & 8.2 & 50.3 & 2.12 & 0.56 & 0.66 & $14 \%$ aperture \\
\hline 1 & 0.45 & 2.2 & 50.3 & 0.95 & 0.20 & 0.89 & $1 \%$ aperture \\
\hline
\end{tabular}

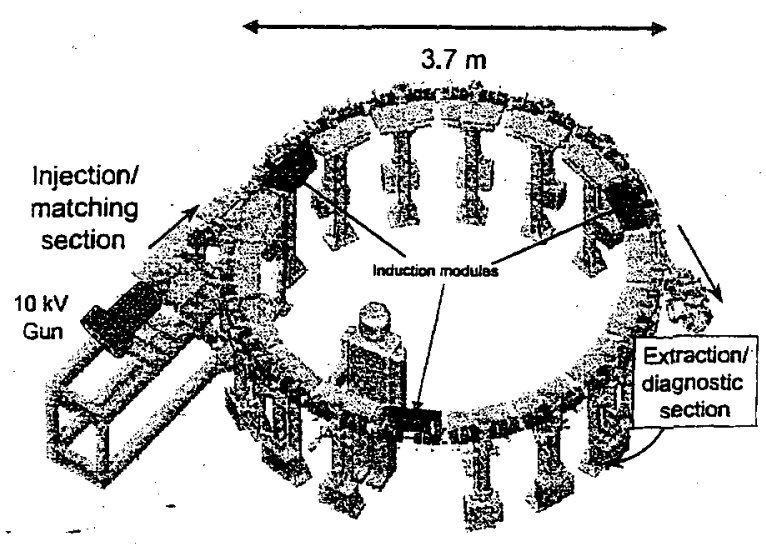

Fig. 2. Layout of the University of Maryland Electron ring.

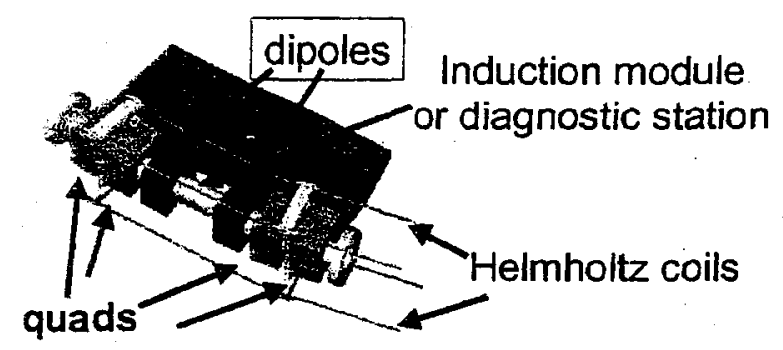

Fig. 3. Detail of the UMER lattice.

repetition rate of $60 \mathrm{~Hz}$ or less from the injector system [7] with the help of two pulsed Panofsky quads and a pulsed dipole [8]. The bunch can be extracted within the first turn or after any number of turns with a system that duplicates the features of the injector line except that the electron gun is replaced by a large diagnostic chamber with phosphor screen, emittance meter and energy analyzer [7].

The operating vacuum is determined by gasscattering-induced emittance growth. The entire vacuum system is designed to be bakeable. All pumping is by ion pumps, one at each diagnostic station, approximately $70 \mathrm{~cm}$ apart; our goal is to reach the low $10^{-9}$ Torr range after bakeout.

\subsection{The electron gun}

The design of the electron gun using the wellknown code EGN has been described elsewhere [7]. It employs a commercial 8-mm diameter dispenser cathode with integral filament and grid. The e-gun includes a micrometer-controlled adjustable $\mathrm{A} / \mathrm{K}$ gap to vary the beam current, a gate valve and an ion pump. A built-in Rogowski-type current monitor is included, as is a rotatable calibrated aperture plate with six masks. The plate includes a pepperpot and a five-beamlet aperture as well as four round apertures ranging from $0.5 \%$ to $100 \%$ transmission. The grid, cathode and grid pulser all float at $10 \mathrm{kV}$ on a conventional high voltage deck. Pulses of $50-100$ ns can be generated using an internal pulse-forming cable that can be changed as desired. The fast switch can be operated to produce either rectangular or parabolic pulses. 


\subsection{Magnet system}

The design of the UMER magnetic elements presented a particular challenge. The uniform magnetic field required to make a $10 \mathrm{keV}$ electron orbit at the radius of UMER $(1.8 \mathrm{~m})$ is $1.5 \mathrm{G}$ about three to five times the value of the vertical component of the earth's magnetic field in the UMER laboratory. The earth's field in the region of the ring has been mapped with milligauss accuracy using a 3-axis flux-gate magnetometer. The vertical component will be used to assist the bending in the ring. The horizontal component is sufficient to deflect the beam about $1 \mathrm{~mm}$ vertically in a half lattice period. Compensation for the horizontal component will be implemented with a series of nine segmented Helmholtz coils placed in a toroidal geometry around the ring. Segmentation of the compensation is necessary because of the sinusoidal variation of the magnetic force from the horizontal with angle around the ring, and because of local variations in the field strength.

When complete the ring magnetic lattice will consist of over 140 quadrupoles, dipoles and steering magnets: The typical focusing gradients and bending fields are on the order of $5 \mathrm{G} / \mathrm{cm}$ and $10 \mathrm{G}$, respectively. The use of iron-based magnets is impractical for such low fields. Therefore, the UMER magnets are based on an iron-free printed circuit (PC) design [9]. These printed circuits are fabricated to a very high tolerance, carefully mounted, measured and then installed and aligned on the beamline. Tables 3 and 4 give the general design characteristics of the PC magnets. The general tolerances on these PC magnets are similar to those of the iron-based magnets of large accelerators. The performance of the magnets on the test bench is summarized in Table 5. While the multipole content meets our design specifications, we are continuing to improve the design, fabrication and testing techniques. The details of the measurement techniques used and the results will be published elsewhere.

\subsection{Diagnostics}

To allow detailed comparison between theory and experiment, UMER will have a comprehen-
Table 3.

Design characteristics of the PC dipoles

\begin{tabular}{ll}
\hline Dipole field & $15.4 \mathrm{G}(5.2 \mathrm{G} / \mathrm{A})$ \\
Current & $3 \mathrm{~A}$ \\
Physical length & $4.4 \mathrm{~cm}$ \\
Effective length & $3.8 \mathrm{~cm}$ \\
Radius & $2.8 \mathrm{~cm}$ \\
Fieid integral & $20 \mathrm{G} \mathrm{cm} / \mathrm{A}$ \\
Resistance & $3 \Omega$ \\
Allowed harmonic content & $<1 \%$ \\
Transverse alignment error & $<0.05 \mathrm{~mm}$ \\
\hline
\end{tabular}

Table 4

Design characteristics of the PC quadrupoles

\begin{tabular}{ll}
\hline Field gradient & $4.1 \mathrm{G} / \mathrm{cm} / \mathrm{A}$ \\
Current & $2 \mathrm{~A}$ \\
Physical length & $4.4 \mathrm{~cm}$ \\
Effective length & $3.6 \mathrm{~cm}$ \\
Radius & $2.8 \mathrm{~cm}$ \\
Field integral & $15 \mathrm{G} / \mathrm{A}$ \\
Resistance (room temp.) & $7 \Omega$ \\
Allowed harmonic content & $<1 \%$ \\
Transverse alignment error & $<0.05 \mathrm{~mm}$ \\
\hline
\end{tabular}

Table 5

Measurements of the relative mutipole components of the PC magnets. The tabulated data represents an average of 20 dipoles, and 20 quadrupoles

\begin{tabular}{lll}
\hline Multipole components & PC dipole & PC quadrupole \\
\hline Dipole & 1 & - \\
Quadrupole & 0.010 & 1 \\
Sextupole & 0.0035 & 0.0035 \\
Octupole & 0.003 & 0.0041 \\
Decapole & 0.0009 & 0.0009 \\
Duodecapole & 0.0011 & 0.0011 \\
\hline
\end{tabular}

sive set of beam diagnostics. Each of the 13 diagnostic stations around the ring will have a phosphor screen and capacitive beam position monitor [10]. In addition, fast current monitors and resistive beam position monitors will also be installed. A sophisticated diagnostic end-chamber has been iabricated by FM Technologies. It houses emittance meters of the slit-wire and pepper-pot 
types, a retarding-field energy analyzer with sub-eV resolution for energy and energy spread measurements, a movable phosphor screen with $1.5 \mathrm{~m}$ travel for insertion into the complete transport line, and a Faraday cup for current measurement.

\section{UMER simulations}

A characteristic feature of the intense beam research at the University of Maryland has been the close coupling between experiment, theory and simulations. To continue this tradition on UMER, we are employing a comprehensive suite of design and simulation codes. These codes are the same as are being used for the design of HIF drivers.

The main code for studying important beam physics is the particle-in-cell (PIC) code WARP [11]. The PIC model, although slow relative to other techniques, has the advantage of a selfconsistent calculation of the seif-fields, which is often necessary for accurate analysis of certain phenomena such as space-charge waves, and instabilities.

-We have benchmarked the WARP code against our beam collimation experiment $[12,13]$. These simulations, which underline the importance of the initial particle distribution to its downstream behavior, exhibit very good agreement with the experiments. We believe that the residual disagreements between our simulation and experiment result from uncertainties in some of the variables (e.g. magnetic fields, initial phase space distributions) in the experiment. Therefore, we plan to ensure that UMER has much improved diagnostics over our previous experiments.

The primary application of the WARP simulations has been' to study the ring design and thoroughly analyze its tolerance to various errors. This effort has been fully intermeshed with the magnet measurements, the electronics specifications, and the alterations to the mechanical design. Details of simulations related to UMER can be found in Ref. [14].

\section{UMER plans}

The UMER project can be divided into four phases as follows:

- Design/Prototype Phase

This phase is now complete (April 2000).

- Construction/Experimental Phase I

Sequential installation of injector and ring section beam physics experiments for about $75 \%$ of one turn. The phase is just beginning and will take three years.

- Experimental Phase II

Ring closure and multi-turn operation.

Low current operation ( $10 \mathrm{~mA}$ ) for 100 turns.

High current operation (100 mA) for at least 10 turns with $\Delta \varepsilon / \varepsilon \leqslant 4$

This phase will begin toward the end of the current grant period.

- Upgrade Phase

Upgrade of UMER to a fast cycling synchrotron to accelerate the beam to $50 \mathrm{keV}$ over 50-100 turns to study resonance crossing.

At the time of writing, April 2000, the UMER electron gun is under test.

\section{Conclusion}

UMER is a low-cost flexible electron model of intense ion recirculators. It offers the opportunity to study the physics of space-charge dominated beams at unprecedented intensity with strong focusing and dispersion. It facilitates the development and benchmarking of HIF driver codes, and is adaptable to many problems in beam physics because of its low cost and modular nature. We anticipate that UMER will uncover a considerable amount of new physics in the unexplored highintensity regime.

\section{Acknowledgements}

We would like to acknowledge our colleagues at Michigan State University, led by Richard York, who have been responsible for the design and 
construction of the main mechanical component on the ring.

This work is supported by the US Department of Energy grant numbers DE-FG02-94ER40855 and DE-FG02-92ER54178.

\section{References}

[1] R.O. Bangarter, Nuovo Cimento Al06 (1993) 1445.

[2] M. Reiser, Theory and Design of Charged Particle Beams, Wiley, New York, 1994, pp. 260-264.

[3] I. Hoffmann, K. Beckert, IEE trans. Nucl. Sci. NS-32 (1985) 2264.

[4] M. Reiser et al., Proceedings of the 1999 IEEE Particle Accelerator Conference, 2000, p. 234.

[5] UMER web site: www.jpr.umd.edu/umer.

[6] Y. Li et al., Calculation of particle motion at the head and tail of a bunch for the University of Maryland Electron Ring, Proceedings of the 1999 IEEE Particle Accelerator Conference, 2000, p. 1656.
[7] D. Kehne et al., Injector for the University of Maryland electron ring (UMER), Nucl. Instr. and Meth. A 464 (2001) 605 , these proceedings.

[8] Y. Li et al., Design, simulation and test of Panofsky quadrupole, Proceedings of the 1999 IEEE Particle Accelerator Conference, 2000, p. 3369.

[9] T. F. Godlove, S. Bernal, M. Reiser, Proceedings of the 1995 Particle Accelerator Conference, 1995, p. 2117.

[10] Y. Zou, et al., Development of a prototype capacitive BPM, Proceedings of the 1999 IEEE Particle Accelerator Conference, 2000, p. 2102.

[11] D.P. Grote et al., Fusion Eng. Des. 32-33 (1996) 193.

[12] S. Bernal, P. Chin, R.A. Kishek, Y. Li, M. Reiser, J.G. Wang, T. Godlove, I. Haber, Phys. Rev. ST Accel. Beams 1 (1998) 044202.

[13] S. Bernal, R.A. Kishek, M. Reiser, I. Haber, Phys. Rev. Lett. 82 (20) (1999) 4002.

[14] R. Kishek et. al., Transverse space charge modes in nonequilibrium beams, Nucl. Instr. and Meth. A 464 (2001) 484 , these proceedings. 


\title{
THE UNIVERSITY OF MARYLAND ELECTRON RING (UMER)*
}

\author{
P.G. O'Shea, B Beaudoin, S. Bernal, Y. Cui, D. Feldman, M. Glanzer, T. Godlove, J. Harris, \\ M. Holland, H. Li, R. Kishek, B. Quinn, N. Rahimi, M. Reiser, A. Valfells, M.Virgo, M. Walter, \\ R. Yun, V.Yun \\ Institute for Research in Electronics and Applied Physics, Univ. of Maryland, College Park, MD \\ 20742-3511, USA \\ D. Kehne, FM Technologies, Fairfax VA, I. Haber, Naval Research Laboratory, Washington DC
}

\begin{abstract}
A detailed understanding of the physics of space-charge dominated beams is vital for many advanced accelerators that desire to achieve high beam intensity. In that regard, low-energy, high-intensity electron beams provide an excellent model system. The University of Maryland Electron ring (UMER), currently under construction, has been designed to study the physics of space-charge dominated beams with extreme intensity in a strong focusing lattice with dispersion. The tune shift in UMER will be more than an order of magnitude greater than exiting synchrotrons and rings. The $10-\mathrm{keV}, 100 \mathrm{~mA}$, UMER beam has a generalized perveance in the range of 0.0015 , and a tune shift of 0.9 . Though compact $(11-\mathrm{m}$ in circumference), UMER is a very complex device, with over 140 focusing and bending magnets. We report on the unique design features of this research facility, the beam physics to be investigated, and early experimental results.
\end{abstract}

\section{INTRODUCTION*}

As beam physicists we strive to develop ever more intense, high quality beams. At the University of Maryland, we have a tradition of using low-energy electron beams as model systems for studying space charge phenomena that are of general interest in intense beam systems [1]. The low-cost, modular nature of our experimental systems has allowed us to develop test stands that are adaptable to many problems in beam physics. Previously, all of our research had been done with straight-line systems. Our motivation to develop a ring system is twofold. There are many interesting phenomena in intense beam physics that evolve over longer distances than our 5-m linear solenoid channel. Furthermore, a ring with both strong focusing and dispersion would also allow us to study resonance issues in a realistic setting.

In linear accelerators, there has been an increased interest in space charge effects in recent years. The introduction of beam with ever lower emittance and increased current, had meant that space-charge driven emittance growth and halo formation can be a consideration at energies as high as high as $100 \mathrm{MeV}$ in

\footnotetext{
Work supported by US Department of Energy
}

some electron linacs proposed for fourth generation light sources [2].

Generally, recirculators and rings have been limited to much lower intensities than linear accelerators. One of the most important limitations in circular machines is the space-charge tune shift. Fear of destructive resonances has constrained existing rings to low intensity and relatively modest tune depression. With space charge, the resonances are no longer caused by the interaction between the single particle orbits and the harmonics of the field errors. Instead, a resonance can occur when the frequency of a collective beam mode coincides with one of the harmonics of the error frequency spectrum. Conventional recirculators (i.e. synchrotrons) have been limited in intensity by the necessity for the beam to make a very large number of orbits.

There has never been an opportunity to perform experiments on such recirculating machines in the region of deep tune depression, and extreme beam intensity. Therefore, almost all of our understanding in this region is based on theory, simulation and conjecture. There is a tremendous need to obtain experimental data in the regime of intensity that exceeds the reach of current machines. UMER [3] is an analog computer for the investigation of phenomena at extreme beam intensity.

The experimental and theoretical study of beam dynamics in UMER will have important applications to high-current circular accelerators other than heavy-ion fusion recirculators; examples of such applications include the low energy end of high intensity electron linacs, ion booster synchrotrons, muon colliders, and spallation neutron sources.

In order to put UMER in a proper context, it is important to define what we mean by intensity, and to illustrate how the increased intensity influences the collective beam motion. We use the dimensionless intensity parameter, $\chi$, as the ratio of the space-charge force to the extemal focusing force at the beam radius $\chi=\frac{K}{k_{0}^{2} a^{2}}$. The space charge term is represented by $K / a$ where $K=\frac{2 I}{I_{0}(\beta \gamma)^{3}}$ is the generalized perveance, $a$ is the $2 x \mathrm{~ms}$ beam radius, $I$ is the beam current, and 
$\mathrm{I}_{0} \approx \frac{\mathrm{mc}^{2}}{30 \mathrm{q}}\left(\mathrm{I}_{0}=3.1 \times 10^{7}(\mathrm{~A} / \mathrm{Z})\right.$ Amps for ions with

charge state $\mathrm{Z}$ and atomic number $A$, and $\mathrm{I}_{0}=17 \mathrm{kA}$ for electrons). The external focusing forces are represented by $k_{0}$, the zero-current betatron wavenumber. These quantities are related to the $4 x \mathrm{rms}$ emittance, $\varepsilon$, through the matched beam envelope equation, in the smooth approximation: $k_{0}^{2}-\frac{K}{a^{2}}=\frac{\varepsilon^{2}}{a^{4}}=k^{2}$ (where $\mathrm{k}$ is the depressed betatron wavenumber) or $1-\chi=\frac{\varepsilon^{2}}{k_{0}^{2} a^{4}}=\frac{k^{2}}{k_{0}^{2}}$. The tune depression can then be expressed in terms of $\chi$ as $\frac{v}{v_{0}}=\frac{k}{k_{0}}=\sqrt{1-\chi}$, and the plasma wavenumber $\mathrm{k}_{\mathrm{p}}$ as $\frac{k_{p}}{k_{0}}=\sqrt{2 \chi}$. For a zero current, fully emittance dominated beam we have $\chi=0$, while $\chi=1$ for a fully space charge dominated beam, with zero emittance. At the lowest values of $\chi$, the motion is dominated by single particle effects and emittance. As $\chi$ approaches unity, collective plasmas oscillations become increasingly dominant. For $\chi=0.5$, the space-charge and emittance terms in the envelope equation are equal, Thus, for the range $0<\chi<.5$, we can say that the beam radius (hence the beam physics) is emittance-dominated; while for $0.5<\chi<1$ the beam radius (physics) is space-charge dominated.

For example, heavy ion fusion drivers will likely operate with $0.89<\chi<0.98$. We see that it is possible to achieve such values of $\chi$ with electrons at $10 \mathrm{keV}, 100$ $\mathrm{mA}, \varepsilon=50 \mu \mathrm{m}$ and $\mathrm{a}=1 \mathrm{~cm}$. Figure 1 shows the range of the intensity parameter for UMER. With $\chi$ ranging from $0.2-0.98$ UMER offers a unique opportunity to study intense beam physics in a completely new regime that begins near the upper intensity range of existing machines. In the space-charge dominated regime of UMER, not only will the tune depression be unprecedented, but also the plasma frequency will exceed the zero-current betatron frequency. This implies that, at the high end of the UMER intensity range, collective effects will have an enormous impact on the beam dynamics.

We should note that a beam in a given machine in places might be either space charge dominated or emittance dominated, depending on the degree of focusing applied. When the beam is large it may be space charge dominated, but when brought to a focus at an interaction point, it may become emittance dominated. Therefore, even in the space charge dominated regime, where the emittance may have only a small influence on the beam envelope evolution, a detailed knowledge of how the emittance is evolving will be necessary in order to understand the behavior of the beam at a final focus [4].

The unknown territory in the extreme space-charge dominated regime will be very challenging and should provide a wealth of new phenomena. The UMER facility will allow us to investigate emittance growth due to conversion of free energy, halo formation, and equipartitioning in a circular machine. So far, these effects have only been studied in linear transport lines. In addition, UMER will permit experimental investigations of longitudinal-transverse coupling and beam profile changes resulting from dispersion; the behavior of bunch ends, resonance traversal; the longitudinal resistive wall instability; and other effects in the space-charge dominated regime that is currently inaccessible.

We are also investigating the possibility of using UMER, and related systems for laboratory studies related to galactic dynamics. Many of the dynamical processes involved in galactic formation and evolution appear to have analogs in the physics of high intensity beams [5].

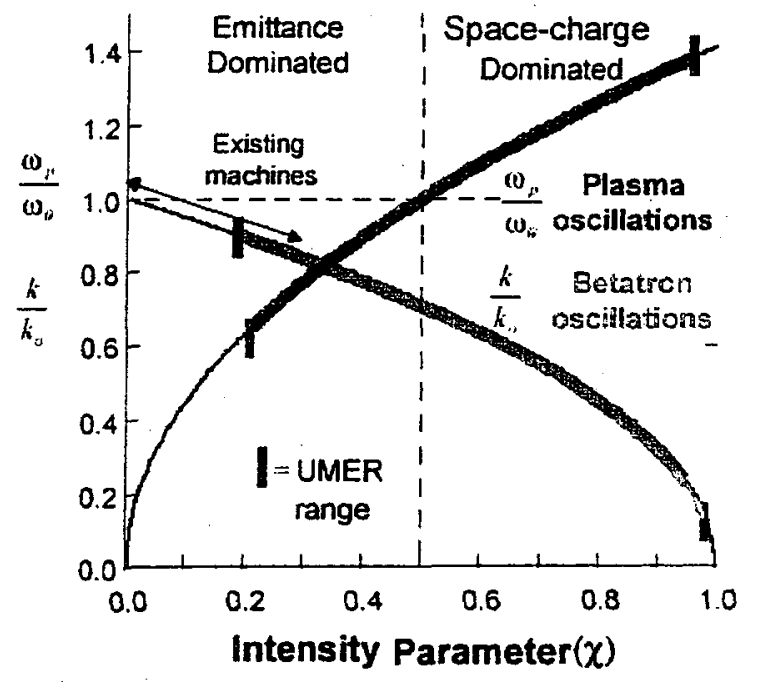

FIGURE 1. Emittance dominated and space-charge dominated regimes, showing the betatron tune depression and plasma tune enhancement with increasing intensity parameter..

\section{UMER DESIGN FEATURES}

In its initial phase UMER will operate at a fixed energy of $10 \mathrm{keV}(\beta=0.2)$. Future upgrades will allow UMER to operate as a fast cycling recirculating accelerator at energies of up to $50 \mathrm{keV}(\beta=0.4)$. Table 1 gives the nominal specifications of UMER at $10 \mathrm{keV}$. The intensity parameier, tune depression or beam current can be varied in UMER over a wide range by changing to different apertures sizes in the beam collimator at the exit of the gun, by changing the anode-cathode spacing, or by changing the beam energy. A complete description of the UMER design features is beyond the scope of this paper. 
Further details can be found on the UNER Web site and in literature cited therein [3].

TABLE 1. UMER design specifications

\begin{tabular}{ll}
\hline Energy & $10 \mathrm{keV}$ \\
$\beta(=\mathrm{v} / \mathrm{c})$ & 0.2 \\
Current & $100 \mathrm{~mA}$ \\
Generalized perveance & $1.5 \times 10^{-3}$ \\
Emittance, $4 \times \mathrm{ms}$, norm & $10 \mu \mathrm{m}$ \\
Pulse Length & $40 \mathrm{~ns}$ \\
Circumference & $11.52 \mathrm{~m}$ \\
Lap time & $197 \mathrm{~ns}$ \\
Pulse repetition. rate & $60 \mathrm{~Hz}$ \\
Mean beam radius & $<1 \mathrm{~cm}$ \\
FODO period & $0.32 \mathrm{~m}$ \\
Zero-current phase advance, $\sigma_{0}$ & $76^{\circ}$ \\
Zero-current Betatron tune, $v_{0}$ & $>7.6$ \\
Tune Depression & $>0.12$ \\
\hline
\end{tabular}

The focusing lattice consists of 36 FODO periods of length $0.32 \mathrm{~m}$ and the ring circumference is $11.52 \mathrm{~m}$. Each FODO section contains two printed-circuit quadrupole magnets and one printed-circuit dipole. A detailed mechanical layout of a full lattice period is shown in Figure 3. The zero-current phase advance per period is $\sigma_{0}=76^{\circ}$, corresponding to a tune of $\nu_{0}=7.6$. The maximum tune depression due to space charge is expected to be between 0.12 and 0.2 . There are 13 diagnostic ports, and three induction modules that provide fast-rising "ear fields" to prevent expansion of the bunch ends and permit acceleration to $50-\mathrm{keV}$ in a future extension of the ring operation:

The electron bunch is injected into the ring at a repetition rate of $60 \mathrm{~Hz}$ or less from the injector system [6] with the help of two pulsed Panofsky quads and a pulsed dipole (see figs. 2,3). The bunch can be extracted within the first turn or after any number of turns with a system that duplicates the features of the injector line except that the electron gun is replaced by a large diagnostic chamber with phosphor screen, emittance meter and energy analyzer $[7,8]$.

The operating vacuum is determined by gas-scatteringinduced emittance growth. The entire vacuum system is designed to be bakeable. All pumping is by ion pumps, one at each diagnostic station, approximately $70 \mathrm{~cm}$ apart; our goal is to reach the low $10^{-9}$ Torr range after bakeout that will be adequate for the $\sim 100$ turns in UMER.

\subsection{Magnet System}

The design of the UMER magnetic elements presented a particular challenge. The uniform magnetic field required to make a $10 \mathrm{keV}$ electron orbit at the radius of UMER (1.8 m) is 1.5 Gauss - about three to five times the value of the vertical component of the earth's magnetic field in the UMER laboratory. The earth's field in the region of the ring has been mapped with milligauss accuracy using a 3-axis flux-gate magnetometer. The vertical component will be used to assist the bending in the ring. The horizontal component is sufficient to deflect the beam about $1 \mathrm{~mm}$ vertically in a half lattice period. Compensation for the horizontal component is implemented with a series of nine segmented Helmholtz coils placed in a toroidal geometry around the ring. Segmentation of the compensation is necessary because of the sinusoidal variation of the magnetic force from the horizontal with angle around the ring, and because of local variations in the field strength.

When complete the ring magnetic lattice will consist of over 140 quadrupoles, dipoles and steering magnets. The typical focusing gradients and bending fields are on the order of $5 \mathrm{Gauss} / \mathrm{cm}$ and 10 Gauss, respectively. The use of iron-based magnets is impractical for such low fields. Therefore, the UMER magnets are based on an iron-free printed circuit (PC) design [9]. These printed circuits are fabricated to a very high tolerance, carefully mounted, measured and then installed and aligned on the beamline. The general tolerances on these PC magnets are similar to those of the iron-based magnets of large accelerators.

A new feature that we have introduced recently is a quadrupole with electronically adjustable skewness (roll). The feature allows us to both correct for residual skewness in our system, and also the deliberately introduce skewness at various location in the ring for beam dynamics studies [10]

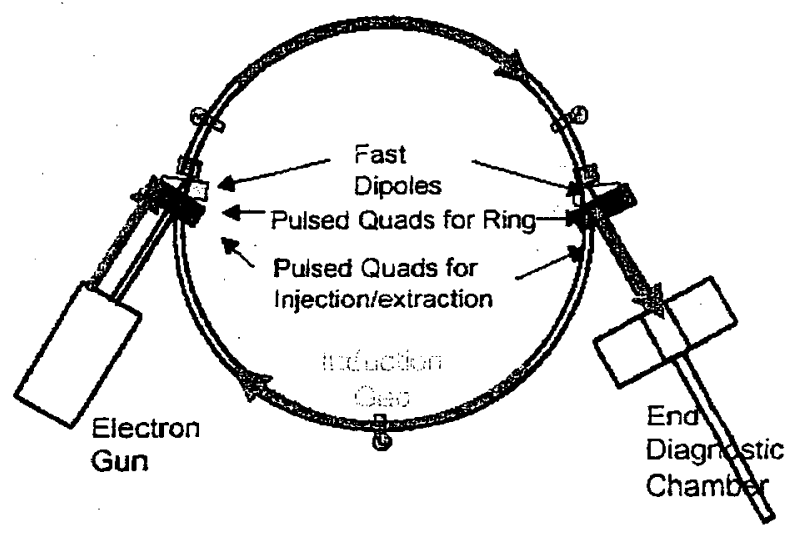

FIGURE 2. UMER schematic showing injection and systems and induction gaps (areey)

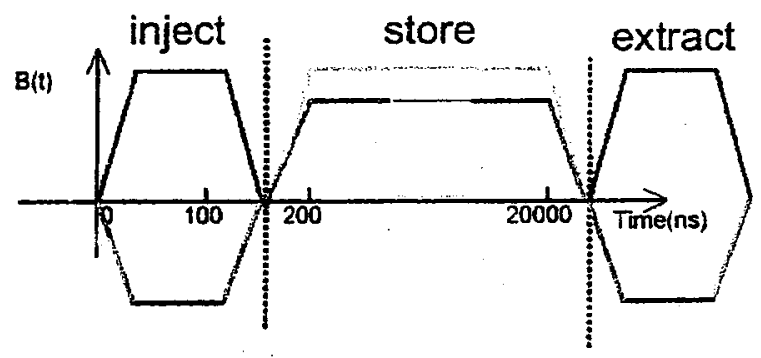

FIGURE 3. Timing schematic for injection and extraction dipoles (red) and Panofsky quadrupoles (blue) 


\subsection{Gun and Injector}

The design of the electron gun and simulations of its performance are described elsewhere $[11,12]$. It employs a commercial $8-\mathrm{mm}$ diameter dispenser cathode with integral filament and grid. The e-gun includes a micrometer-controlled adjustable $A-K$ gap to vary the beam current, a gate valve and an ion pump. A built-in Rogowski-type current monitor is included, as is a rotatable calibrated aperture plate with six masks. The plate includes a pepperpot and a five-beamlet aperture as well as four round apertures ranging from $0.5 \%$ to $100 \%$ transmission. Pulses of 50-100 ns with various pulse shapes can be generated.

In addition, we have been investigating the possibility of producing ultra-short ( $1 \mathrm{~ns}$ ) current pulses on top of the main pulse. We have achieved this by using a nitrogen laser as a photocathode drive laser on the dispenser cathode [13]. This will allow is to create perturbations on the beam that are well located in time and space. The evolution of such a perturbation will allow us to study dissipative processes, e.g. resistive wall phenomena.

The remainder of the injector consists of the transport channel with a solenoid immediately after the gun followed by 5 quadrupoles that lead to the pulsed injection kicker system (figs.3, 4).

\subsection{Diagnostics Controls and Alignment}

To allow detailed comparison between theory and experiment,- UMER will have a comprehensive set of beam diagnostics. Each of the 13 diagnostic stations around the ring will have a phosphor screen and capacitive beam position monitor [14]. In addition, fast current monitors and resistive beam position monitors will also be installed. A sophisticated diagnostic end-chamber has been fabricated by FM Technologies. The chamber houses emittance meters of the slit-wire and pepper-pot types; a retarding-field energy analyzer with $\mathrm{eV}$ resolution for energy and energy spread measurements; a movable phosphor screen with 1.5 meter travel for insertion into the complete transport line; and a Faraday cup for current measurement.

Details of alignment and controls are provide in a companion paper [15]

\section{INITIAL EXPERIMENTAL RESULTS}

\subsection{Emittance}

The initial emittance characterization is by the pepper pot method (fig, 4 ), which has shown that the beam has a $4 \mathrm{x}$ normalized emittance of $15 \mu \mathrm{m}$. The emittance data has yet to be evaluate in detail; however, fig. 4 indicates that the local temperature of the beam varies with radius.

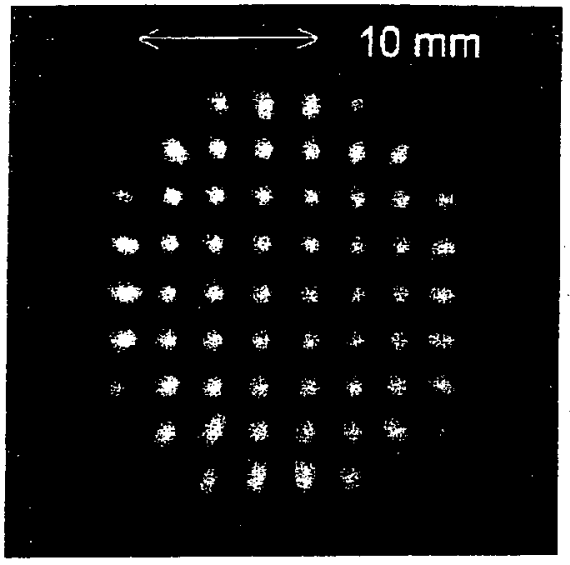

FIGURE 4. Pepperpot image of the beam from the UMER gun

\subsection{Energy Spread}

We have developed an energy an electrostatic retarding voltage energy analyzer [Valfells, Cui]. Simulations show the resolution of the device is about $8 \mathrm{eV}$, and the measured values of the energy spread are just slightly greater than this value(fig. 6).

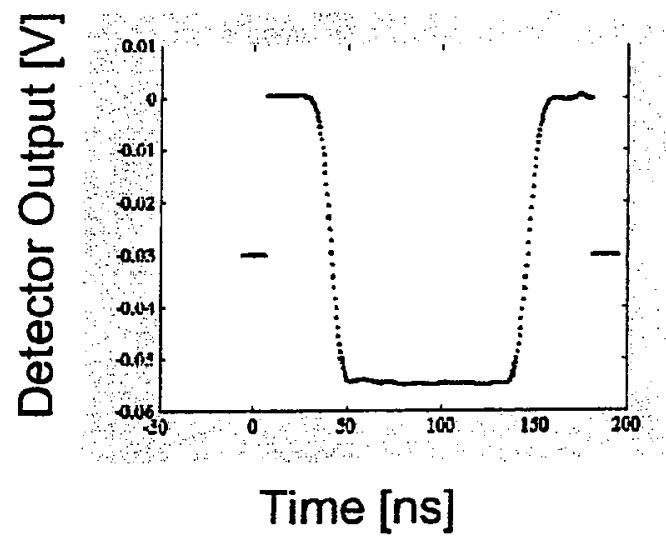

Figure 5 Current pulse on energy analyzer with zero bias voltage

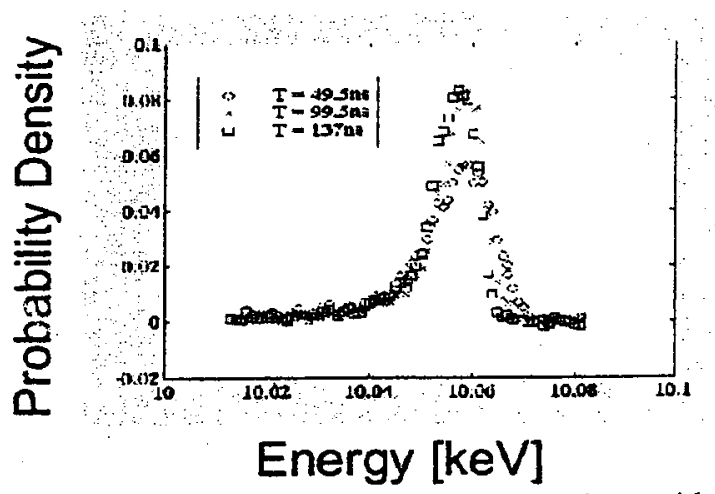

Figure 6 Energy Spread measured at three times with the current pulse in fig 5 
While the measured energy spread meets our specification of $20 \mathrm{eV}$, we are continuing to improve our diagnostic. We have designed an improved energy analyzer with a resolution of $0.5 \mathrm{eV}$ (cui).

\subsection{Matching}

The beam in the injector has been configures to meet the specifications for matching into the ring (fig. 7)
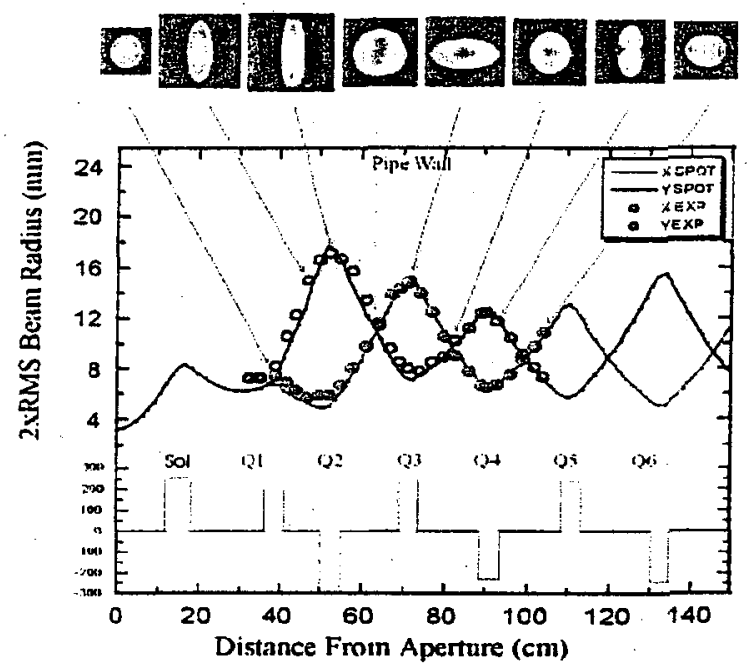

Figure 7 Simulation (solid) and experimental data (points) for the $2 x$ RMS beam radius along the injector

\section{PLANS}

At present, (June 2000) the UMER injector is going through final testing. Injection into the first turn of the ring will commence shortly.

The remaining phases of the UMER project are as follows:

- Construction/Experimental Phase I

Sequential installation of injector and ring section beam physics experiments for about $75 \%$ of one turn. The phase is just beginning (August 2000) and will take three years.

- Experimental Phase II

Ring closure and multi-tum operation.

Low current operation ( $10 \mathrm{~mA})$ for 100 turns.

High current operation (100 mA) for at least 10 turns

with $\frac{\Delta \varepsilon}{\varepsilon} \leq 4$

This phase will begin toward the end of the current grant period

- Upgrade Phase
Upgrade of UMER to a fast cycling synchrotron to accelerate the beam to $50 \mathrm{keV}$ over 50-100 turns to study resonance crossing.

\section{ACKNOWLEDGEMENTS}

We wish to acknowledge of Richard York and his colleagues at Michigan State University the assistance with the design and construction for UMER components.

\section{REFERENCES}

[1] Martin Reiser, "Theory and Design of Charged Particle Beams", (New York: John Wiley \& Sons, Inc., 1994)

[2] P.G. O'Shea and H.P. Freund, Science, 2921854 (2001)

[3] UMER Web: http://www.ireap.umd.edu/umer

[4] R.A. Kishek, P.G. O'Shea, M. Reiser, Physical Review Letters. 85, 4514 (2000)

[5] R. Kishek at al., "Computational Investigation of Dissipation and Reversibility of Space Charge Driven Phenomena in Beams", these proceedings

[6] S. Bernal et al.,"Beam Tests of the 10-kV Injector for UMER", these proceedings

[7] A. Valfells et al., "Energy Analyzer Experiments on UMER", these proceedings

[8] Y. Cui et al., Studies of Energy Spread Growth???, these proceedings[zhang]

[9] W.W. Zhang, H. Li, S. Bernal, T. Godlove, R.A Kishek, P.G. O'Shea, M. Reiser, M. Venturini, V. Yun, Physical Review ST-AB 3, 122401 (2000)

[10] $\mathrm{H}$. Li et al.,"Printed Circuit Magnets for UMERNew Developments

[11] D. Kehne, et al "Injector for the University of Maryland Electron Ring (UMER)" to appear in Nucir. Instr. Meth

[12] I Haber et al., "Computer Simulations of the UMER injector" these proceedings

[13] D. Feldman et al, "Combined Thermionic and Photoelectric Emission from Dispenser Cathodes", these proceedings

[14] J. Harris, "A fast Beam Position Monitor for UMER", these proceedings

[15] M. Virgo et al., Controls and alignment for UMER, these proceedings 


\title{
Compact high-resolution retarding field energy analyzer for space-charge-dominated electron beams
}

\author{
Y. Zou, Y. Cui, V. Yun, A. Valfells, R. A. Kishek, S. Bernal, I. Haber, M. Reiser, and P. G. O'Shea \\ Institute for Research in Electronics and Applied Physics, University of Maryland, College Park, Maryland 20742 \\ J. G. Wang \\ SNS, Oak Ridge National Laboratory, Oak Ridge, Tennessee 3783 I
}

(Received 22 April 2002; published 5 July 2002)

\begin{abstract}
We have developed a compact high-resolution retarding field energy analyzer for measuring the energy spread of space-charge-dominated electron beams. This energy analyzer has a cylindrical electrode to overcome the defocusing effects due to space-charge forces, beam trajectories, aperture effect, etc. The device provides excellent spatial and temporal information on the beam energy spread. Single-particle simulation shows that this energy analyzer has very good resolution for low-energy electron beams of several kilovolts and with large divergence angles. The energy analyzer has been tested with $2.5 \mathrm{keV}$, $60 \mathrm{~mA}$ electron beams. The measured energy spread is also compared with the theoretical calculations taking into account two main energy spread sources, namely, the Boersch effect and the longitudinallongitudinal relaxation.
\end{abstract}

DOI: 10.1 103/PhysRevSTAB.5.072801

PACS numbers: 29.30.Dn, 29.27.Fh, 29.17.+w

\section{INTRODUCTION}

Advanced particle accelerators for heavy-ion inertial fusion, high-energy colliders, free electron lasers, and other applications require high-quality, intense beams, which can be focused onto a small diameter target. For such applications, the beams must have very low emittance and low-energy spread. While there have been many experimental and theoretical investigations of emittance growth in intense beams, very little work exists on the sources and evolution of energy spread in such beams. At the University of Maryland, various experiments have been carried out to study space-charge-dominated electron beams. Some of these experiments, such as the resistive-wall instability experiment [1] and the University of Maryland Electron Ring (UMER) [2] currently being constructed, require knowledge of the beam's energy spread. The initial energy spread of the beams from the gun with a thermionic emitter and the growth of this energy spread in the transport line must be measured with high precision. For these applications, a high-resolution energy analyzer is necessary. The work described here deals with measurements of beams from thermionic sources only; however, in the near future we plan to extend our exploration to include photoemitters also.

In order to gain a full understanding of the evolution of the energy spread, we require a device that has excellent spatial and temporal resolution such that we can develop a 3D map of the energy spread in the beam. This is important because the energy spread may vary from head to tail as well as transversely in the beam bunch. Because of its simplicity and high signal-to-noise ratio output, the retarding field energy analyzer becomes a natural choice for low-energy electron beams. In parallel plate structures, however, space-charge forces, beam trajectories, mechani- cal misalignment, beam mismatching, etc., often lead to poor resolution of the energy measurement. In this paper, we report on the design and testing results of a cylindrical retarding field energy analyzer, which has greatly improved the resolution compared to a parallel plate energy analyzer used previously in our experiments. In the following sections, we first discuss the theory of the energy spread source in the space-charge-dominated beams and then we will describe the design, simulation, and beam tests of the new retarding field energy analyzer.

\section{SOURCES OF BEAM ENERGY SPREAD FROM A THERMIONIC GUN}

There are many sources contributing to the longitudinal energy spread for an electron beam emitted from a thermionic electron gun. The two dominant sources for the energy spread are the Boersch effect [3] and the longitudinallongitudinal relaxation effect [4]. The Boersch effect is due to the energy transfer from the transverse direction to the longitudinal direction. The longitudinal-longitudinal effect is due to the fast beam acceleration in the electron gun and therefore the energy transfer from potential energy to the thermal kinetic energy. Here we outline the calculations of the beam energy spread due to these two sources.

First, consider the Boersch effect following Ref. [5]. When the beam is emitted from the cathode, it has an inherent energy spread due to the cathode temperature. The rms beam energy spread is related to the cathode temperature by

$$
\Delta E_{\mathrm{rms}}=m v_{\mathrm{rms}}^{2}=k_{B} T .
$$

Here $v_{\mathrm{ms}}$ is the rms thermal velocity, $k_{B}$ is the Boltzmann constant, and $T$ is the beam temperature. For a typical gun with cathode temperature of $1100^{\circ} \mathrm{C}$, the corresponding 
thermal energy is around $0.1 \mathrm{eV}$. For beams emitted from photocathodes, this number may be larger, perhaps a few tenths of an electron volt.

When the beams are accelerated by a static electric field, every particle gains the same longitudinal kinetic energy. Consider two particles initially having an energy difference $\Delta E$; after acceleration they will still have the same amount of energy difference. However, their velocity difference and, therefore, the temperature of the beams will change. It can be shown that the beam temperature $T_{\| f}$ after acceleration is given by [5]

$$
k_{B} T_{\| f}=\left(k_{B} T_{\| i}\right)^{2} /\left(2 q V_{0}\right)
$$

Here $T_{\| i}$ is the initial beam temperature, which is isotropic (i.e., $T_{\| i}=T_{1 i}=T_{i}$ ) before the acceleration and $V_{0}$ is the accelerating voltage. This effect is known as the longitudinal cooling effect due to the acceleration.

Note that the transverse temperature $T_{\perp f}$ is the same as the initial temperature since the acceleration acts only in the longitudinal direction. The longitudinal temperature, which is determined by Eq. (2), becomes negligibly small compared to the transverse temperature. The beam is now in an extremely anisotropic state $\left(T_{\| f} \ll T_{\perp f}\right)$. Coulomb collisions and other processes will try to force the beam velocity distribution into thermal equilibrium. The resulting beam longitudinal energy spread will increase. This thermal relaxation process is referred to as the Boersch effect.

The transverse-longitudinal temperature relaxation effect is very complicated in a real beam. For a simple case in which the beam propagates through a smooth focusing channel and has a constant radius, the temperature relaxation can be described by the following equations [5]:

$$
T_{\perp},-2 / 3 T_{\perp i}\left(1+0.5 e^{-3 t / \tau_{\text {eff }}}\right)
$$

and

$$
T_{\|}=2 / 3 T_{\perp i}\left(1-e^{-3 t / \tau_{\mathrm{eff}}}\right),
$$

where $\tau_{\text {eff }}$ is the effective temperature relaxation time and is given by.

$$
\tau_{\text {eff }}=4.44 \times 10^{20}\left(k_{B} T_{\text {eff }} / m c^{2}\right)^{3 / 2} /(n \ln \Lambda) .
$$

Here $T_{\text {eff }}$ is related to $T_{1 i}$ by

$$
k_{B} T_{\text {eff }}=0.373 k_{B} T_{\perp i}
$$

$\ln \Lambda$ is the Coulomb logarithm and is obtained from

$$
\ln \Lambda=\ln \left[5.66 \times 10^{21}\left(k_{B} T / m c^{2}\right)^{3 / 2} n^{-1 / 2}\right] .
$$

In the above equation, $n$ is the particle density given by

$$
n=I /\left(e a^{2} \pi v\right)
$$

where $I$ is the beam current, $a$ is the beam radius, and $v$ is the beam velocity. From Eq. (4), we find that the final longitudinal and transverse temperature will reach the equilibrium state at two thirds of the initial transverse temperature. The time it takes to reach equilibrium is about $\tau_{\text {eff }}$.
The energy transfer from the transverse direction to the longitudinal direction due to the Boersch effect can be suppressed by applying a strong axial magnetic field. However, even if this energy transfer is suppressed, the final beam energy spread is still found to be larger than what is predicted by the transverse-longitudinal cooling effect. The reason is that the Coulomb collisions in addition to the Boersch effect cause another temperature relaxation called longitudinal-longitudinal relaxation [4]. If the acceleration is fast (nonadiabatic) compared to the period of the electron beam plasma oscillation period, the initial state of relative high density and slow motion of electrons relative to each other is preserved during the acceleration process. Coulomb collisions tend to relax this nonequilibrium state into a more homogenous state and result in energy spread increase. The criteria for the adiabatic condition is determined by a dimensionless parameter $\lambda$, which compares the cooling time $\left(-1 / T \times d t_{\|} / d t\right)^{-1}$ with the plasma period $\omega_{p}^{-1}$ and is given by the formula [4]

$$
\lambda=-1 /\left(\omega_{p} T\right) \frac{d T_{\|}}{d t} .
$$

If $\lambda<1$, then the acceleration is adiabatic. $\lambda$ depends on the acceleration structure. It is interesting to note that a thermal electron gun with Pierce geometry has $\lambda$ equal to $2^{3 / 2}$, a nonadiabatic case [4], which applies to our experiment.

Combining both the transverse-longitudinal Boersch effect and the longitudinal-longitudinal relaxation effect, the final beam energy spread can be expressed as

$$
\Delta \tilde{E}_{\| f}=\left[\frac{1}{\pi \varepsilon_{0}} q n^{1 / 3} q V_{0}+2 q V_{0} k_{B} T_{\|}\right]^{1 / 2}
$$

Here $\Delta \tilde{E}_{\| f}$ is the rms energy spread after acceleration and subsequent beam propagation; $q V_{0}$ is the beam energy and $T_{\|}$is an increasing function of time or distance of beam propagation, which can be calculated from Eq. (4) (Boersch effect). All the beam energies are in units of eV; $\boldsymbol{n}$ is the beam density and $\boldsymbol{q}$ is the electron charge. The first term in the bracket corresponds to the longitudinallongitudinal effect, and the second term is the transverse-longitudinal (Boersch) effect. The longitudinallongitudinal effect is stronger in the initial acceleration and propagation phase when there is a density and longitudinal temperature gradient. The energy spread due to the Boersch effect, on the other hand, increases monotonically until the longitudinal temperature reaches equilibrium. After a certain time of propagation, the Boersch effect will become the dominant source of the energy spread.

\section{DESIGN AND SIMULATION OF THE RETARDING FIELD ENERGY ANALYZER}

There are several types of retarding field energy analyzers as described in Ref. [6], which reviews retarding field analyzers with geometries of the parallel plate, spherical condenser, and Faraday cage [6]. The most commonly 
used one is the parallel plate energy analyzer, which consists of two parallel plates. This kind of device has been used for the UMER project in the past experiments [7]. The first plate is the grounding plate and the second one is biased to a negative high voltage for retardation of electron beams. Only those particles that have a longitudinal kinetic energy higher than the retarding voltage can pass through the second electrode and introduce a current signal at the collector. This structure has good resolution only for a beam with trajectories parallel to the axis of the energy analyzer. In practice, due to space-charge forces, beam emittance, and misalignment, etc., the beam particles always have finite divergence angles. The diverging particle trajectories lead to poor resolution. Our modi fication to this structure, in which the retarding voltage plate is replaced with a cylindrical electrode, has signi ficantly improved the energy resolution [8]. As will be shown below, the computer simulation predicts that the resolution of the cylindrical energy analyzer is $0.6 \mathrm{eV}$ for a $2.5 \mathrm{keV}$ beam, corresponding to a relative resolution of $0.024 \%$. Another feature of this retarding field energy analyzer is its compact size. With a length of $4.3 \mathrm{~cm}$ and a diameter of $5.1 \mathrm{~cm}$, this analyzer can be easily inserted at any place in the beam line.

Figure 1 shows the schematic of the energy analyzer. The electron beam comes from the left. The first plate is a grounded plate with a circular aperture through which a small beamlet passes into the high voltage region. Because the energy analyzer samples a small fraction of the full beam, the beamlet in the energy analyzer is much less space charge dominated than the whole beam. The aperture is covered with a metal mesh to ensure the uniformity of electrical fields near the grounded plate. The high voltage cylinder is supported by two Macor rings and has a feedthrough to connect to the external high voltage source. The rear end of the high voltage cylinder is also covered with a wire mesh. Behind the high-voltage cylinder is a copper collector, from which the current signal is picked up by a $50 \Omega$ BNC connector. The whole structure is shielded

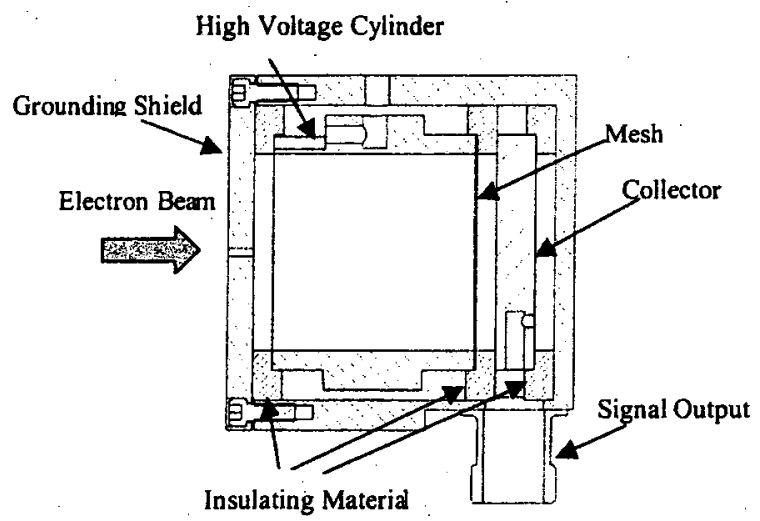

FIG. 1. Schematic of the energy analyzer with cylindrical high voltage electrode. The length is $4.3 \mathrm{~cm}$ and the diameter is $5.1 \mathrm{~cm}$. The aperture diameter is $1 \mathrm{~mm}$. to ensure good signal-to-noise ratio. Because of the tight space of the diagnostic chamber to hold the energy analyzer, the collector is a single plate instead of a regular Faraday cup structure. The secondary electron emission is suppressed due to the reverse electric field on the surface of the collector.

A computer code called SIMION was used to simulate the performance of the energy analyzer. SIMION is a PC-based charged particle optics simulation program. A wire mesh can also be incorporated in the program. Figure 2 depicts the simulated equipotential lines and the typical beam trajectories in the energy analyzer. The curved equipotential lines help to focus the beam. Figure 3 illustrates beam trajectories in this energy analyzer at different retarding voltages. The apparent voids in the beam in each figure are due to the limited number of beamlets in the simulation. The depicted beam is monoenergetic at an energy of $2.5 \mathrm{keV}$. The initial beam radius is $0.5 \mathrm{~mm}$, which is determined by the size of the aperture in the front plate. The divergence angle of the beam is $5^{\circ}$. Figure $3(a)$ is for a retarding voltage of $2.4994 \mathrm{kV}$, in which case all beam particles can pass through and appear as the current signal at the collector. Figure 3 (b) is for a retarding voltage of $2.4997 \mathrm{kV}$, where some particles are re flected back while some particles still can pass through, depending on the divergence of their trajectories. When the retarding voltage is raised to $2.500 \mathrm{kV}$, all particles are re flected and the analyzer acts as a mirror, as shown in Fig. 3(c). Figure 4 shows the simulated energy analyzer output versus the retarding voltage. The triangular points are the simulated data points and the curves connecting the simulated data points are simple straight lines for illustrating purposes.

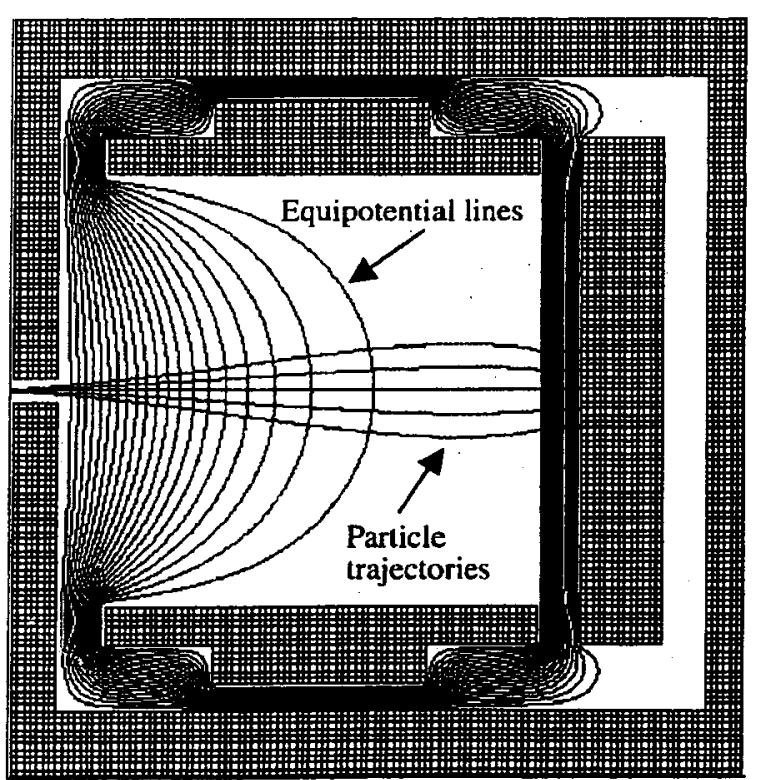

FIG. 2. Equipotential lines and typical beam trajectories in the energy analyzer. 
(a) Retarding voltage is $2.4994 \mathrm{kV}$

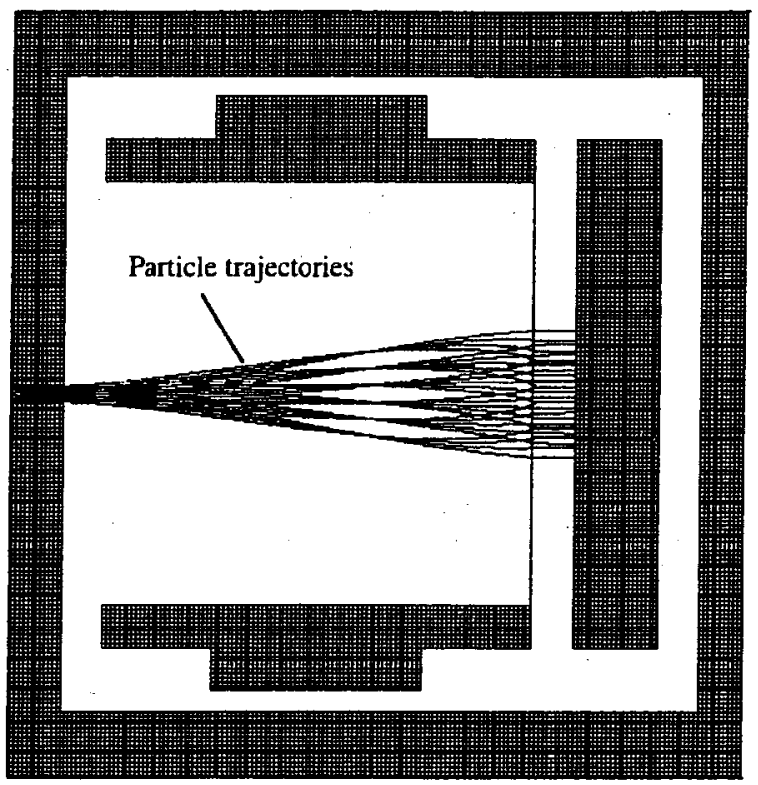

(b) Retarding voltage is $2.4997 \mathrm{kV}$

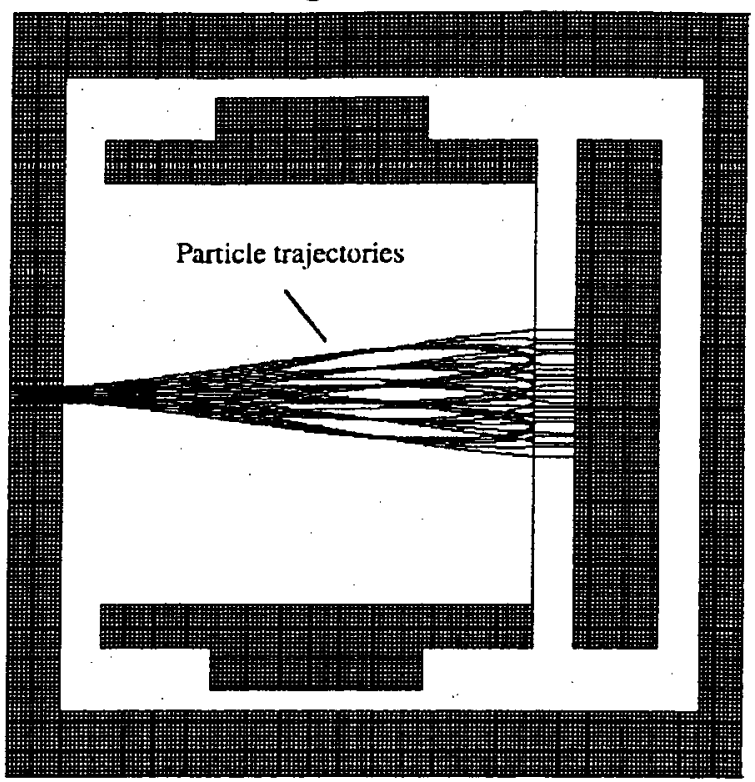

(c) Retarding voltage is $2.500 \mathrm{kV}$

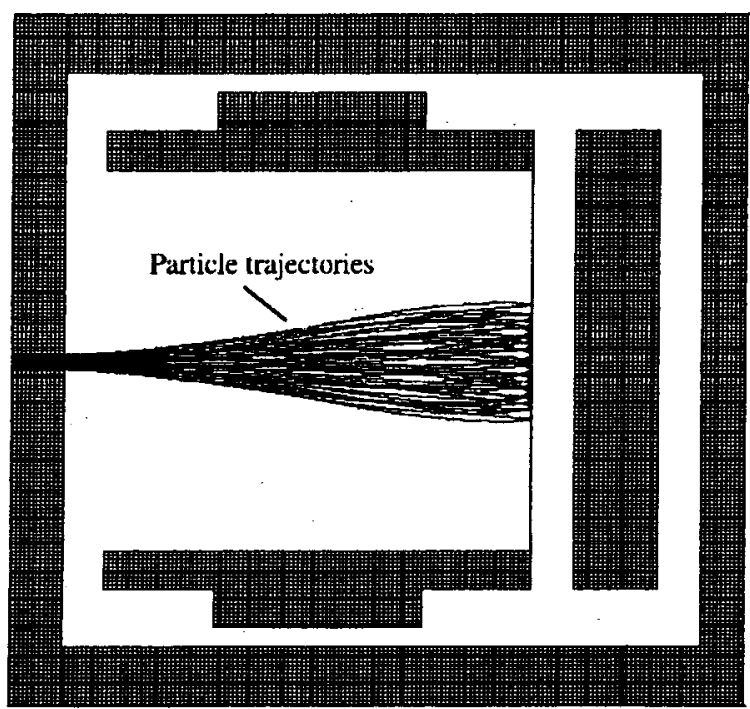

FIG. 3. Particle trajectories at different retarding voltages.

The ideal response of the energy analyzer has a sharp cutoff at $2.5 \mathrm{kV}$. For this energy analyzer, the curve starts to drop off at $2.4994 \mathrm{kV}$, giving the resolution of the device of around $0.6 \mathrm{eV}$. The curve is totally cut off at $2.5 \mathrm{kV}$, which corresponds to the total beam energy.

To compare the performance of the cylindrical analyzer to that of the parallel plate energy analyzer, we also simulated the performance of a parallel plate energy analyzer with the same beam parameters. The response of the parallel plate energy analyzer is also plotted in Fig. 4. The figure shows that the resolution of the parallel plate energy analyzer is around $20 \mathrm{eV}$. From this comparison, we can see the significant improvement of the resolution of the cylindrical energy analyzer compared to that of a parallel plate structure.

It is interesting to note that the performance of the device depends on the various beam parameters, for example, the beam kinetic energy $E_{k}$. Figure 5 depicts the simulations of the performance of the cylindrical energy analyzer with beam energies of $2.5,5.5$, and $10 \mathrm{keV}$. The resolution 


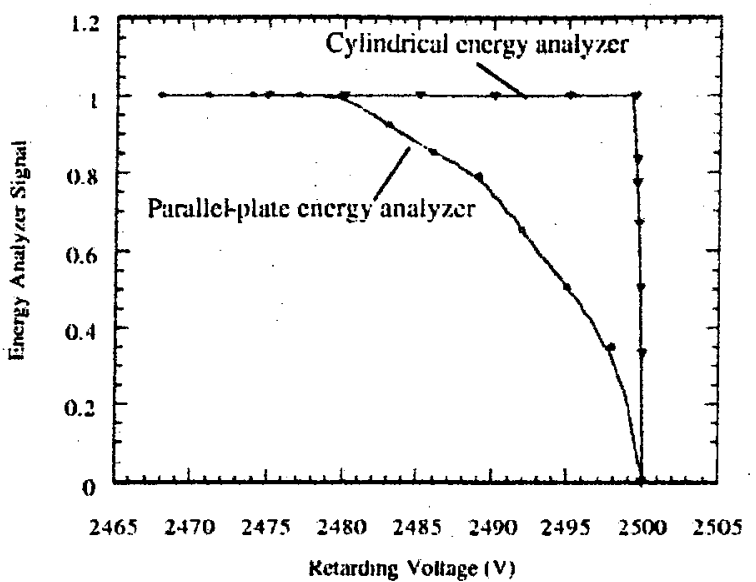

FIG. 4. (Color) Simulation shows that, for the beam with divergence angle of $5^{\circ}$, the cylindrical energy analyzer has a resolution of $0.6 \mathrm{eV}$, while the parallel plate counterpart has a resolution of $20 \mathrm{eV}$.

at $10 \mathrm{keV}$ is around $2.2 \mathrm{eV}$, worse than the $2.5 \mathrm{keV}$ case. The reason that the energy analyzer has poor resolution at higher beam energy is as follows. The Euler trajectory equations in a conserved system can be expressed as [5]

$$
r^{\prime \prime}=\left(1+r^{\prime 2}\right)\left(\partial \phi / \partial r-r^{\prime} \partial \phi / \partial z\right) /(2 \phi) .
$$

Here $\phi$ is the electrostatic potential at a certain position corresponding to the particle's kinetic energy at that point. It can be shown from this equation that the radial position $r(z)$ and the slope $r^{\prime}(z)=v_{r} / v_{z}$ of a particle trajectory in an axisymmetric electrostatic field, such as that in our energy analyzer, remain the same at any given distance $z$ along the axis if the electrostatic potentials are changed by the same factor at every point and the beam

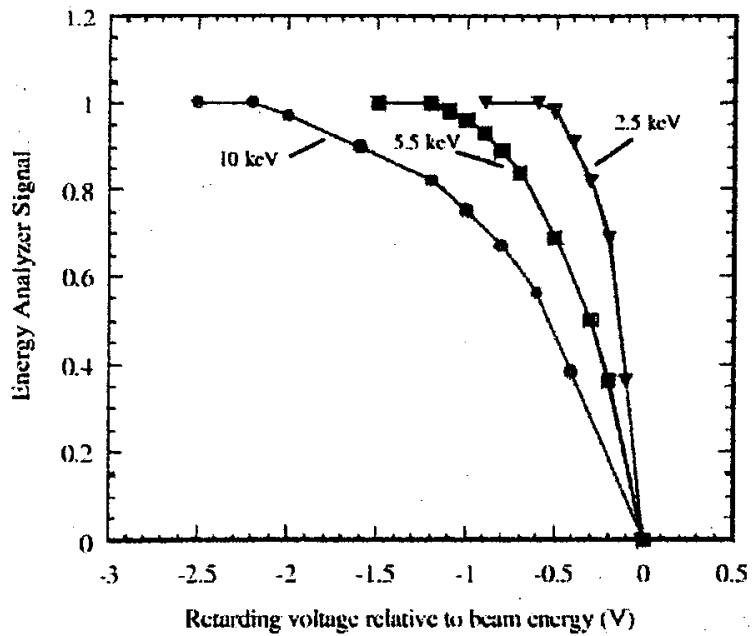

FIG. 5. (Color) Energy analyzer response curves to a monoenergetic beam at different beam energies of $2.5,5.5$, and $10 \mathrm{keV}$, respectively. initial conditions $\left[r\left(z_{0}\right)\right.$ and $\left.r^{\prime}\left(z_{0}\right)\right]$ remain the same. Since $v_{r} / v_{z}=\tan (\theta)$, then the difference between the longitudinal kinetic energy $E_{z}$ and the total kinetic energy $E_{k}$, $\Delta E$, is given by

$$
\Delta E=E_{k} \sin ^{2}(\theta) .
$$

Since $\theta$ remains constant under the above scaling rules as $E_{k}$ increases, it follows that the resolution of the electrostatic energy analyzer, $\Delta E$, is proportional to $E_{k}$. In another words, if we define the relative resolution $\Delta E / E$, then it is a constant independent of the total beam energy.

\section{BEAM TEST OF THE ENERGY ANALYZER}

The experimental setup, as shown in Fig. 6, consists of a gridded thermionic electron gun, three solenoidal magnetic lenses, and a diagnostic chamber. The energy analyzer is in the diagnostic chamber located between the second and the third solenoids. The third solenoid is after the energy analyzer and consequently, it has no effect for the beam trajectories in the energy analyzer. The distances of the first two solenoids and the energy analyzer from the electron gun are 9.1, 21.6, and $33.2 \mathrm{~cm}$, respectively. The solenoidal magnetic field extends less than $10 \mathrm{~cm}$ from the center, so there is practically no magnetic field inside the energy analyzer. Figure 7 depicts a typical beam envelope in the transport line calculated from the K-V envelope equation. We can see that the energy analyzer is located at the waist of the beam. The energy analyzer can be aligned with respect to the beam by a linear and rotation feedthrough.

Figure 8 is a typical output signal from the energy analyzer for a beam with an energy of $5 \mathrm{keV}$ and a pulse length of $100 \mathrm{~ns}$. From the signal, we can see that this energy analyzer has a very fast rise time $(\sim 2 \mathrm{~ns})$ and can reproduce the beam waveform faithfully. This is essential because some of the experiments conducted at the University of Maryland require time resolved energy spread from the beam. The way to measure the energy spread of the beam is to adjust the retarding voltage. As the retarding voltage is increased, the energy analyzer output becomes smaller. By differentiating the energy analyzer output voltage with respect to the retarding voltage, we can get the beam energy profile information. Figures 9 and 10 show such a case. In Fig. 9, different traces corresponding to different retarding voltages are plotted together. The numbers on the traces correspond to increasing retarding voltages. Note that the beam energy spread information is time resolved. We can see that, at the front end of the beam, the particle energy is larger than that in the main beam due to the longitudinal space-charge force. From this figure, we can construct the beam energy profile by taking the difference of the voltage of traces corresponding to the different retarding voltages. To study the energy spread of the main beam particles, we set the sampling point at the middle of the beam pulse. Figure 10 shows the energy profile. The 


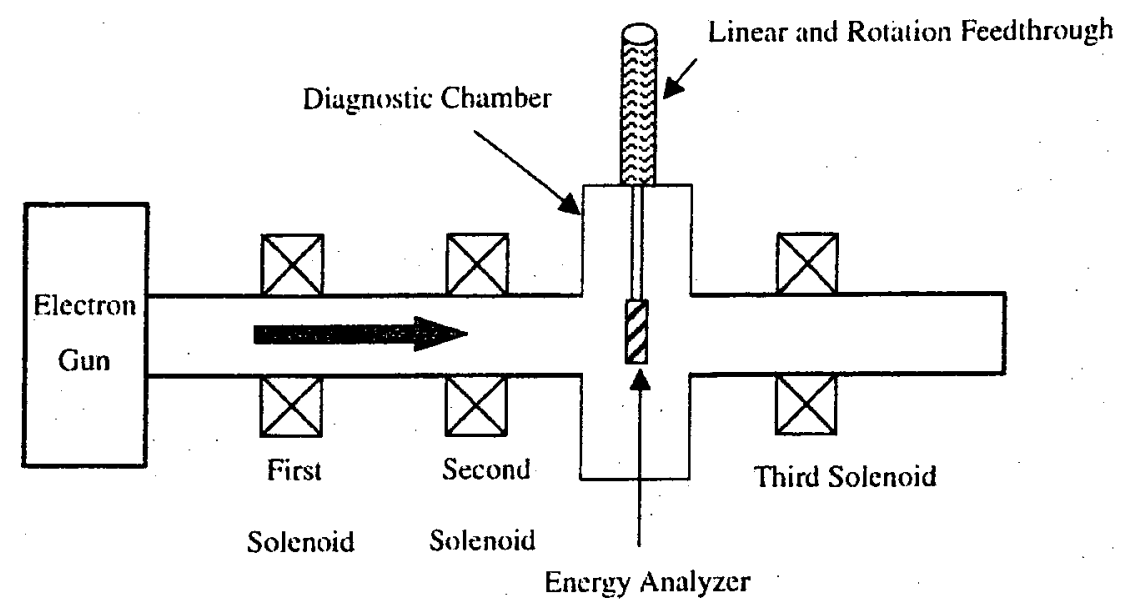

FIG. 6. Experimental setup for the energy analyzer test.

experimental data points and their fit curve are plotted together in this figure. The error bars are also shown here. The magnitudes of the error bars are determined by the signal fluctuations due to the background noise. Note that the energy distribution shown here is asymmetric and has a tail at the high-energy side. More study is underway to explain the source of this tail. In this experiment, the beam energy and current are $2.5 \mathrm{keV}$ and $60 \mathrm{~mA}$, respectively, which is strong space charge dominated. Because of the focusing forces introduced by the cylindrical structure, the defocusing forces due to the space-charge forces and other factors have been well balanced. The measured beam rms energy spread is $1.8 \mathrm{eV}$.

It is interesting to compare the experimental results with the theoretical predictions of the electron beam energy spread. In our example, the beam energy is $2.5 \mathrm{keV}$. The beam is emitted from a cathode with a radius of $5 \mathrm{~mm}$. In the transport system, the average beam radius is around $9 \mathrm{~mm}$. The first term of Eq. (6) gives the energy spread due to the longitudinal-longitudinal effect, which is $0.9 \mathrm{eV}$ in this case. The second term of Eq. (6) gives the rms energy spread due to the Boersch effect, which is $\sim 1.0 \mathrm{eV}$. The

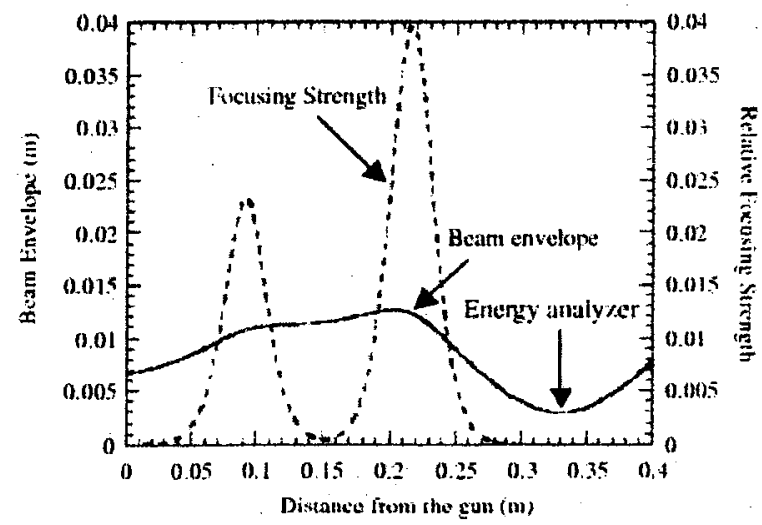

FIG. 7. (Color) Typical beam envelope in the transport line. final energy spread resulting from both effects as given by Eq. (6) is $\sim 1.3 \mathrm{eV}$, which is very close to the experimental result, $1.8 \mathrm{eV}$.

From the comparison, we find that the experimental result is larger than the theoretical predictions. There are several possible reasons for this discrepancy. First, as we explained earlier, the finite resolution of the energy analyzer will contribute to the larger measured beam energy spread. The computer simulation shows that the resolution of this energy analyzer is $0.6 \mathrm{eV}$ for a $2.5 \mathrm{keV}$ beam. In reality, because of the collective space-charge forces and Coulomb scattering of electron beams inside the energy analyzer, the resolution of the energy analyzer might be worse than the computer simulation. A two-dimensional particle-in-cell code simulation is underway to study the behavior of the energy analyzer in more detail. Second, besides these two main energy spread sources mentioned here, there are possible other sources, which may cause an increase of the beam energy spread. In the study reported here, we have considered only the Coulomb scattering theory. However, in space-charge-dominated beams,

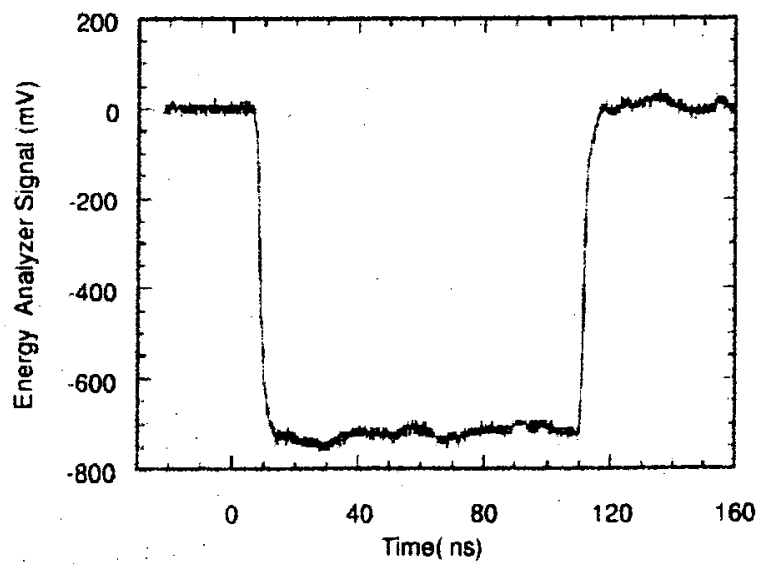

FIG. 8. (Color) Typical energy analyzer output signal. 


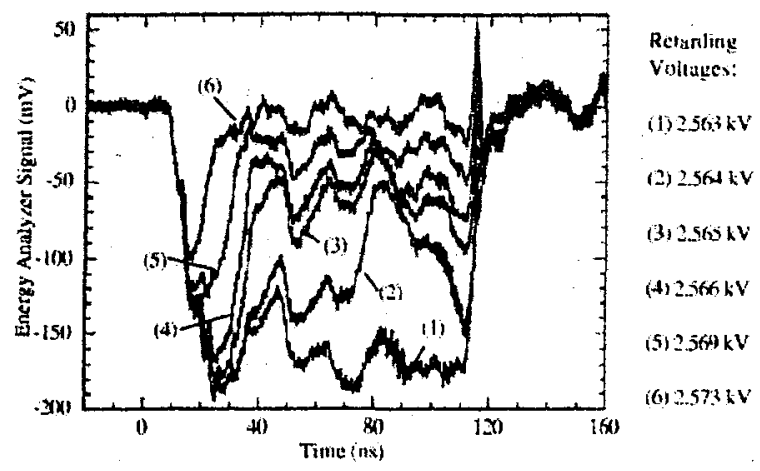

FIG. 9. (Color) Energy analyzer outputs at different retarding voltages.

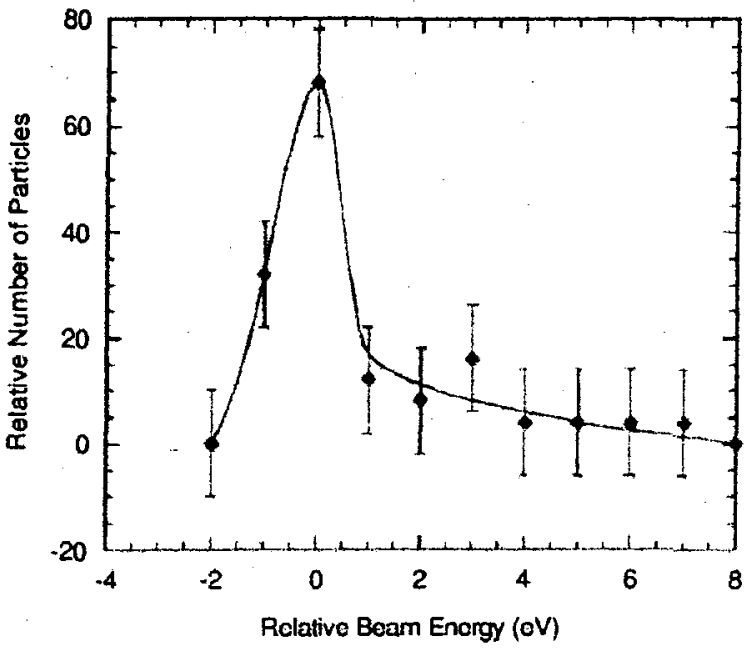

FIG. 10. (Color) Beam energy profile for a beam with energy of $2.5 \mathrm{keV}$ and current of $60 \mathrm{~mA}$. The rms energy width is $1.8 \mathrm{eV}$.

collective instabilities may produce relaxation towards 3D thermal equilibrium. The larger energy spread observed in the experiments may in fact be due to collective instabilities and other effects that will have to be studied in future simulation work. Actually, there are already some simulations in the literature, showing that a beam with a two-temperature anisotropy $\left(T_{\| i} \ll T_{\perp i}\right)$ will develop instability, which may increase the longitudinal energy spread and even significantly deteriorate the beam quality [9-11].

\section{CONCLUDING REMARKS}

A compact high-resolution electrostatic energy analyzer for low-energy electron beams has been designed, simulated, and beam tested. The simulations and beam tests show that the energy analyzer has very good resolution for the energy measurement of space-charge-dominated electron beams in the energy range of several thousand electron volts. The measured beam energy spread is very close to the predictions of a theoretical model based on a longitudinal beam cooling effect, transverse-longitudinal, and longitudinal-longitudinal temperature relaxation effects. A new version of this analyzer, which has higher resolution for the $10 \mathrm{keV}$ UMER beam has been constructed and is currently being tested [12].

Last, it should be noted that our electrostatic analyzer is applicable only to low-energy electron and ion beams. At higher energies, other techniques have to be used to measure the energy spread of the beams.

\section{ACKNOWLEDGMENTS}

This work is supported by DOE Grants No. DEFG0292ER54178 and No. DEFG0294ER40855.

[1] J. G. Wang, H. Suk, and M. Reiser, Phys. Rev. Lett. 79, 1042 (1997).

[2] P. G. O'Shea, M. Reiser, R. A. Kishek, S. Bernal, H. Li, M. Pruessner, V. Yun, Y. Cui, W. Zhang, Y. Zou, T. Godlove, D. Kehne, P. Haldemann, and I. Haber, Nucl. Instrum. Methods Phys. Res., Sect. A 464, 646 (2001).

[3] H. Boersch, Z. Phys. 139, 115 (1954).

[4] A.V. Aleksandrov et al., Phys. Rev. A 46, 6628 (1992).

[5] M. Reiser, Theory and Design of Charged Particle Beams (Wiley, New York, 1994).

[6] J.A. Simpson, Rev. Sci. Instrum.32, 1283 (1961).

[7] H. Suk, Ph.D. Thesis, University of Maryland, 1996.

[8] Y. Zou, Ph.D. thesis, University of Maryland, 2000.

[9] A. Friedman, D. P. Grote, and I. Haber, Phys. Fluids B4, 2203 (1992).

[10] 1. Haber, D. A. Callahan, A. Friedman, D. P. Grote, and A.B. Langdon, Fusion Eng. Des.32, 169-174 (1996).

[11] I. Haber, D. A. Callahan, A. Friedman, D. P. Grote, and S. M. Lund, Nucl. Instrum. Methods Phys. Res., Sect. A 415, 405 (1998).

[12] Y. Cui, A. Valfells, S. Bernal, M. Reiser, P.G. OShea, R. A. Kishek, V. Yun, and N. Rahimi, inProceedings of the 2001 Particle Accelerator Conference, Chicago, IL,edited by Peter W. Lucas and Sara Webber (IEEE, Piscataway, NJ, 2001). 


\title{
Compact high-resolution retarding field energy analyzer for space-charge-dominated electron beams
}

\author{
Y.'Zou, Y. Cui, V. Yun, A. Valfells, R. A. Kishek, S. Bernal, I. Haber, M. Reiser, and P. G. O'Shea \\ Institute for Research in Electronics and Applied Physics, University of Maryland, College Park, Maryland 20742 \\ J.G. Wang \\ SNS, Oak Ridge National Laboratory, Oak Ridge, Tennessee 37831
}

(Received 22 April 2002; published 5 July 2002)

\begin{abstract}
We have developed a compact high-resolution retarding field energy analyzer for measuring the energy spread of space-charge-dominated electron beams. This energy analyzer has a cylindrical electrode to overcome the defocusing effects due to space-charge forces, beam trajectories, aperture effect, etc. The device provides excellent spatial and temporal information on the beam energy spread. Single-particle simulation shows that this energy analyzer has very good resolution for low-energy electron beams of several kilovolts and with large divergence angles. The energy analyzer has been tested with $2.5 \mathrm{keV}$, $60 \mathrm{~mA}$ electron beams. The measured energy spread is also compared with the theoretical calculations taking into account two main energy spread sources, namely, the Boersch effect and the longitudinallongitudinal relaxation.
\end{abstract}

DOI: 10.1103/PhysRevSTAB.5.072801

PACS numbers: 29.30.Dn, 29.27.Fh, 29.17.+w

\section{INTRODUCTION}

Advanced particle accelerators for heavy-ion inertial fusion, high-energy colliders, free electron lasers, and other applications require high-quality, intense beams, which can be focused onto a small diameter target. For such applications, the beams must have very low emittance and low-energy spread. While there have been many experimental and theoretical investigations of emittance growth in intense beams, very little work exists on the sources and evolution of energy spread in such beams. At the University of Maryland, various experiments have been carried out to study space-charge-dominated electron beams. Some of these experiments, such as the resistive-wall instability experiment [1] and the University of Maryland Electron Ring (UMER) [2] currently being constructed, require knowledge of the beam's energy spread. The initial energy spread of the beams from the gun with a thermionic emitter and the growth of this energy spread in the transport line must be measured with high precision. For these applications, a high-resolution energy analyzer is necessary. The work described here deals with measurements of beams from thermionic sources only; however, in the near future we plan to extend our exploration to include photoemitters also.

In order to gain a full understanding of the evolution of the energy spread, we require a device that has excellent spatial and temporal resolution such that we can develop a 3D map of the energy spread in the beam. This is important because the energy spread may vary from head to tail as well as transversely in the beam bunch. Because of its simplicity and high signal-to-noise ratio output, the retarding field energy analyzer becomes a natural choice for low-energy electron beams. In parallel plate structures, however, space-charge forces, beam trajectories, mechani- cal misalignment, beam mismatching, etc., often lead to poor resolution of the energy measurement. In this paper, we report on the design and testing results of a cylindrical retarding field energy analyzer, which has greatly improved the resolution compared to a parallel plate energy analyzer used previously in our experiments. In the following sections, we first discuss the theory of the energy spread source in the space-charge-dominated beams and then we will describe the design, simulation, and beam tests of the new retarding field energy analyzer.

\section{SOURCES OF BEAM ENERGY SPREAD FROM A THERMIONIC GUN}

There are many sources contributing to the longitudinal energy spread for an electron beam emitted from a thermionic electron gun. The two dominant sources for the energy spread are the Boersch effect [3] and the longitudinallongitudinal relaxation effect [4]. The Boersch effect is due to the energy transfer from the transverse direction to the longitudinal direction. The longitudinal-longitudinal effect is due to the fast beam acceleration in the electron gun and therefore the energy transfer from potential energy to the thermal kinetic energy. Here we outline the calculations of the beam energy spread due to these two sources.

First, consider the Boersch effect following Ref. [5]. When the beam is emitted from the cathode, it has an inberent energy spread due to the cathode temperature. The rms beam energy spread is related to the cathode temperature by

$$
\Delta E_{\mathrm{rms}}=m v_{\mathrm{ms}}^{2}=k_{B} T .
$$

Here $v_{\mathrm{ms}}$ is the rms thermal velocity, $k_{B}$ is the Boltzmann constant, and $T$ is the beam temperature. For a typical gun with cathode temperature of $1100^{\circ} \mathrm{C}$, the corresponding 
used one is the parallel plate energy analyzer, which consists of two parallel plates. This kind of device has been used for the UMER project in the past experiments [7]. The first plate is the grounding plate and the second one is biased to a negative high voltage for retardation of electron beams. Only those particles that have a longitudinal kinetic energy higher than the retarding voltage can pass through the second electrode and introduce a current signal at the collector. This structure has good resolution only for a beam with trajectories parallel to the axis of the energy analyzer. In practice, due to space-charge forces, beam emittance, and misalignment, etc., the beam particles always have finite divergence angles. The diverging particle trajectories lead to poor resolution. Our modi fication to this structure, in which the retarding voltage plate is replaced with a cylindrical electrode, has signi ficantly improved the energy resolution [8]. As will be shown below, the computer simulation predicts that the resolution of the cylindrical energy analyzer is $0.6 \mathrm{eV}$ for a $2.5 \mathrm{keV}$ beam, corresponding to a relative resolution of $0.024 \%$. Another feature of this retarding field energy analyzer is its compact size. With a length of $4.3 \mathrm{~cm}$ and a diameter of $5.1 \mathrm{~cm}$, this analyzer can be easily inserted at any place in the beam line.

Figure 1 shows the schematic of the energy analyzer. The electron beam comes from the left. The first plate is a grounded plate with a circular aperture through which a small beamlet passes into the high voltage region. Because the energy analyzer samples a small fraction of the full beam, the beamlet in the energy analyzer is much less space charge dominated than the whole beam. The aperture is covered with a metal mesh to ensure the uniformity of electrical fields near the grounded plate. The high voltage cylinder is supported by two Macor rings and has a feedthrough to connect to the external high voltage source. The rear end of the high voltage cylinder is also covered with a wire mesh. Behind the high-voltage cylinder is a copper collector, from which the current signal is picked up by a $50 \Omega \mathrm{BNC}$ connector. The whole structure is shielded

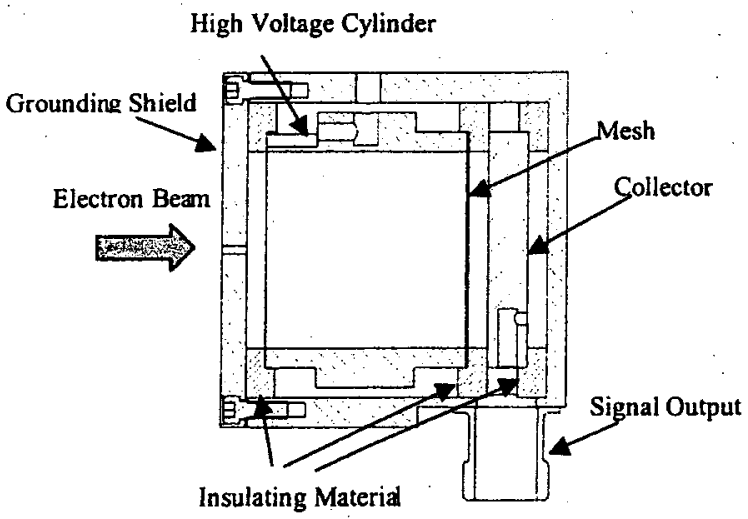

FIG. 1. Schematic of the energy analyzer with cylindrical high voltage electrode. The length is $4.3 \mathrm{~cm}$ and the diameter is $5.1 \mathrm{~cm}$. The aperture diameter is $1 \mathrm{~mm}$. to ensure good signal-to-noise ratio. Because of the tight space of the diagnostic chamber to hold the energy analyzer, the collector is a single plate instead of a regular Faraday cup structure. The secondary electron emission is suppressed due to the reverse electric field on the surface of the collector.

A computer code called SIMION was used to simulate the performance of the energy analyzer. SIMION is a PC-based charged particle optics simulation program. A wire mesh can also be incorporated in the program. Figure 2 depicts the simulated equipotential lines and the typical beam trajectories in the energy analyzer. The curved equipotential lines help to focus the beam. Figure 3 illustrates beam trajectories in this energy analyzer at different retarding voltages. The apparent voids in the beam in each figure are due to the limited number of beamlets in the simulation. The depicted beam is monoenergetic at an energy of $2.5 \mathrm{keV}$. The initial beam radius is $0.5 \mathrm{~mm}$, which is determined by the size of the aperture in the front plate. The divergence angle of the beam is $5^{\circ}$. Figure 3(a) is for a retarding voltage of $2.4994 \mathrm{kV}$, in which case all beam particles can pass through and appear as the current signal at the collector. Figure 3(b) is for a retarding voltage of $2.4997 \mathrm{kV}$, where some particles are re flected back while some particles still can pass through, depending on the divergence of their trajectories. When the retarding voltage is raised to $2.500 \mathrm{kV}$, all particles are re flected and the analyzer acts as a mirror, as shown in Fig. 3(c). Figure 4 shows the simulated energy analyzer output versus the retarding voltage. The triangular points are the simulated data points and the curves connecting the simulated data points are simple straight lines for illustrating purposes.

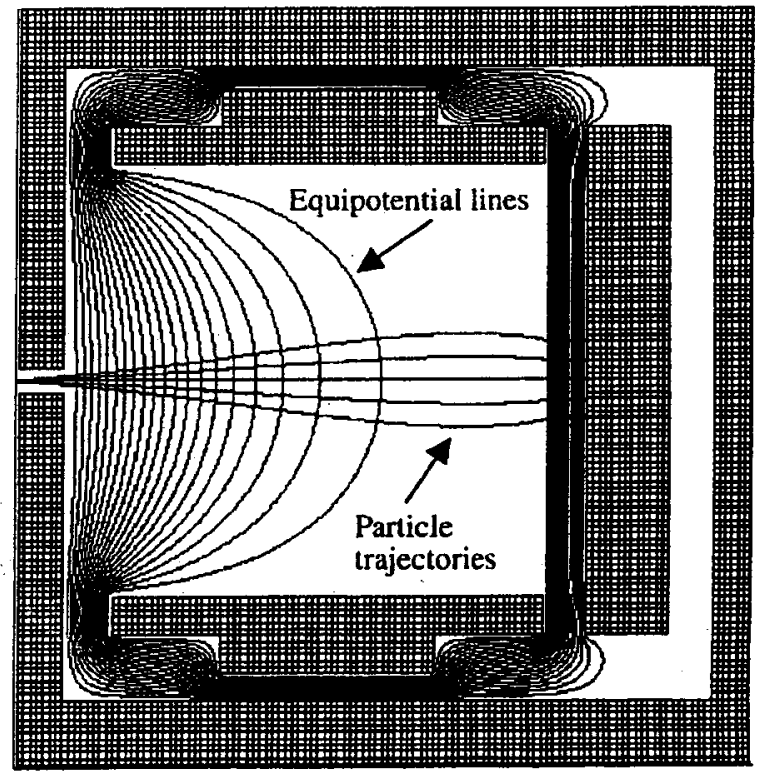

FIG. 2. Equipotential lines and typical beam trajectories in the energy analyzer. 
(a) Retarding voltage is $2.4994 \mathrm{kV}$

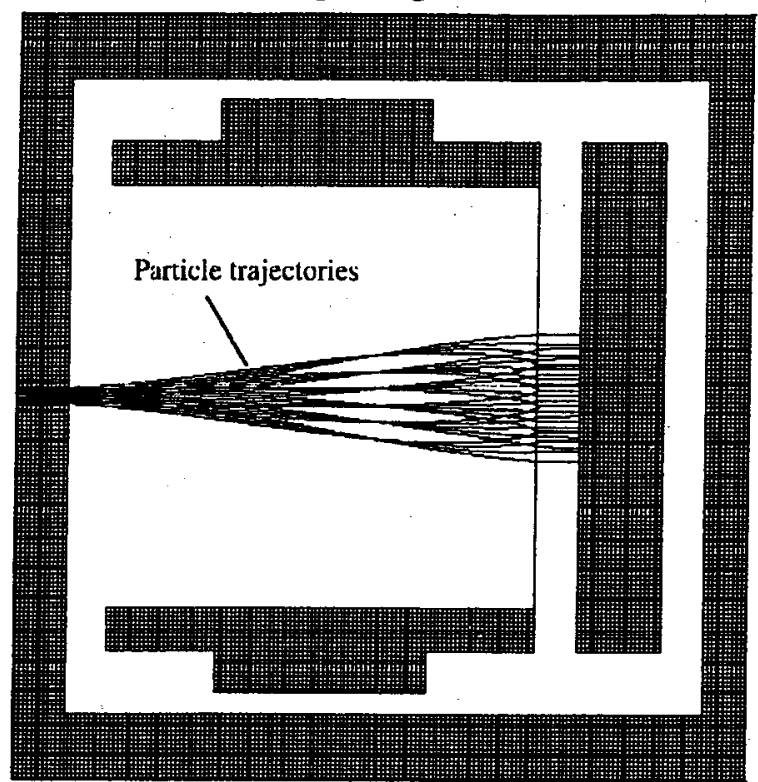

(b) Retarding voltage is $2.4997 \mathrm{kV}$

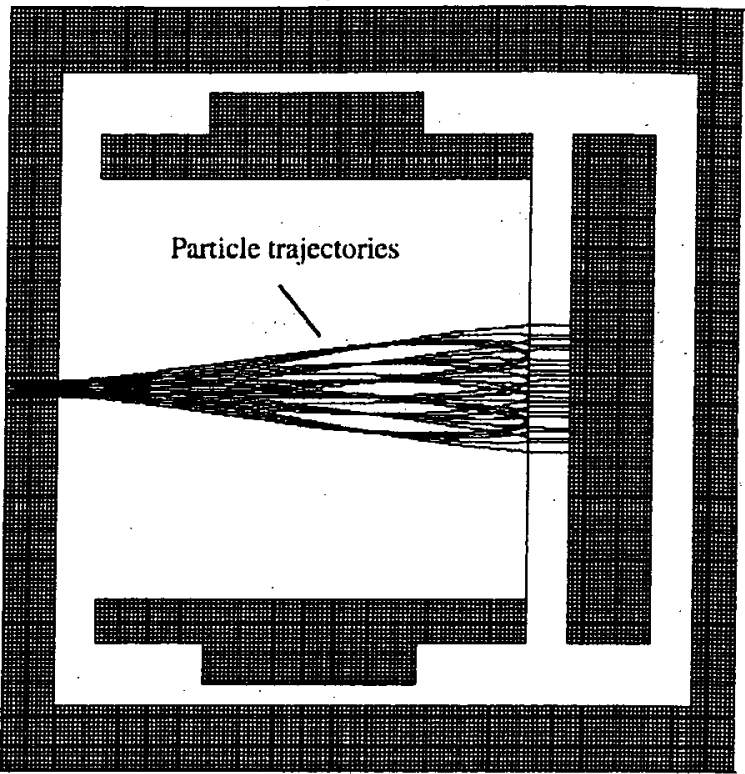

(c) Retarding voltage is $2.500 \mathrm{kV}$

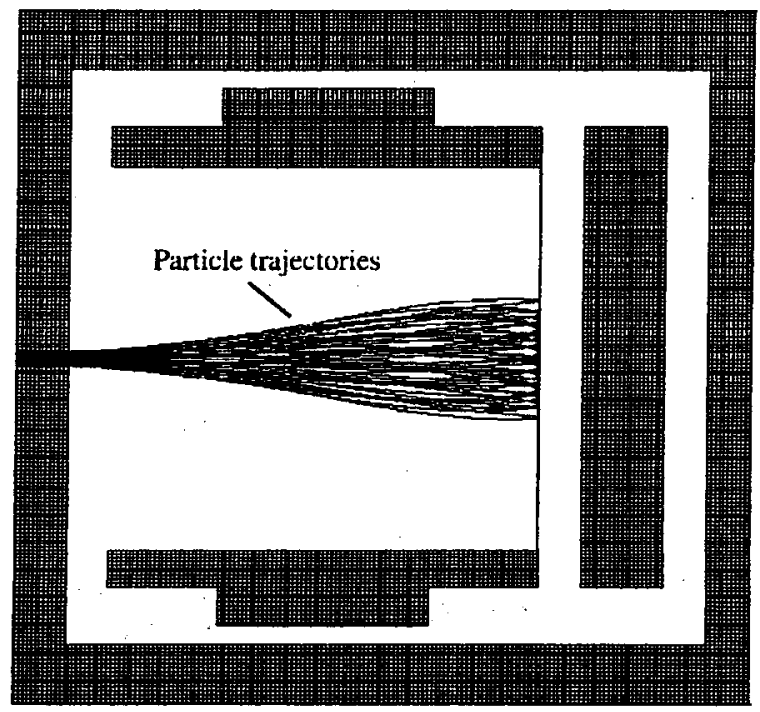

FIG. 3. Particle trajectories at different retarding voltages.

The ideal response of the energy analyzer has a sharp cutoff at $2.5 \mathrm{kV}$. For this energy analyzer, the curve starts to drop off at $2.4994 \mathrm{kV}$, giving the resolution of the device of around $0.6 \mathrm{eV}$. The curve is totally cut off at $2.5 \mathrm{kV}$, which corresponds to the total beam energy.

To compare the performance of the cylindrical analyzer to that of the parallel plate energy analyzer, we also simulated the performance of a parallel plate energy analyzer with the same beam parameters. The response of the parallel plate energy analyzer is also plotted in Fig. 4. The figure shows that the resolution of the parallel plate energy analyzer is around $20 \mathrm{eV}$. From this comparison, we can see the significant improvement of the resolution of the cylindrical energy analyzer compared to that of a parallel plate structure.

It is interesting to note that the performance of the device depends on the various beam parameters, for example, the beam kinetic energy $E_{k}$. Figure 5 depicts the simulations of the performance of the cylindrical energy analyzer with beam energies of $2.5,5.5$, and $10 \mathrm{keV}$. The resolution 


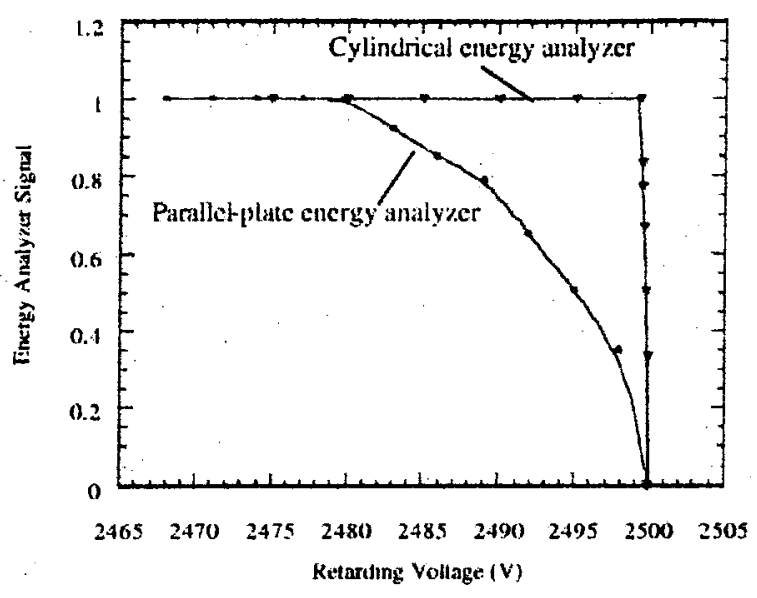

FIG. 4. (Color) Simulation shows that, for the beam with divergence angle of $5^{\circ}$, the cylindrical energy analyzer has a resolution of $0.6 \mathrm{eV}$, while the parallel plate counterpart has a resolution of $20 \mathrm{eV}$.

at $10 \mathrm{keV}$ is around $2.2 \mathrm{eV}$, worse than the $2.5 \mathrm{keV}$ case. The reason that the energy analyzer has poor resolution at higher beam energy is as follows. The Euler trajectory equations in a conserved system can be expressed as [5]

$$
r^{\prime \prime}=\left(1+r^{\prime 2}\right)\left(\partial \phi / \partial r-r^{\prime} \partial \phi / \partial z\right) /(2 \phi) .
$$

Here $\phi$ is the electrostatic potential at a certain position corresponding to the particle's kinetic energy at that point. It can be shown from this equation that the radial position $r(z)$ and the slope $r^{\prime}(z)=v_{r} / v_{z}$ of a particle trajectory in an axisymmetric electrostatic field, such as that in our energy analyzer, remain the same at any given distance $z$ along the axis if the electrostatic potentials are changed by the same factor at every point and the beam

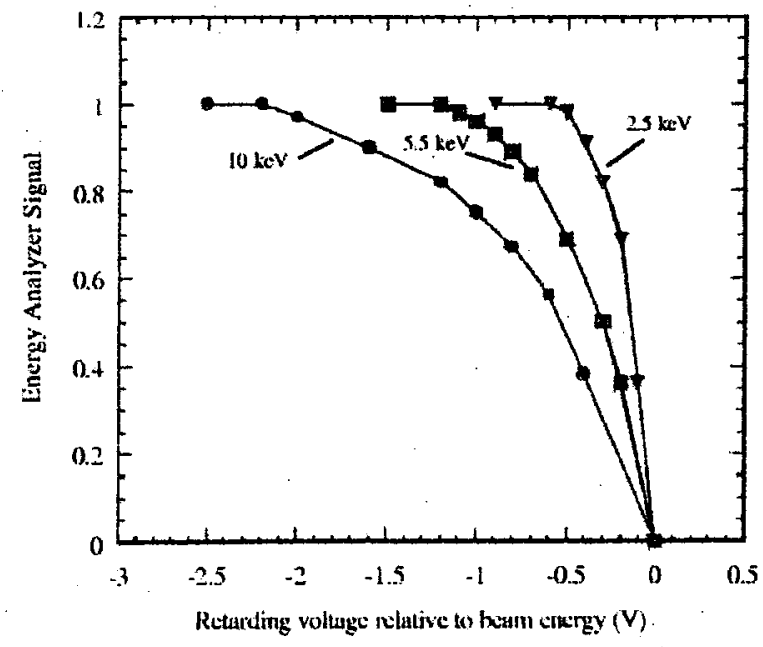

FIG. 5. (Color) Energy analyzer response curves to a monoenergetic beam at different beam energies of $2.5,5.5$, and $10 \mathrm{keV}$, respectively. initial conditions $\left[r\left(z_{0}\right)\right.$ and $\left.r^{\prime}\left(z_{0}\right)\right]$ remain the same. Since $v_{r} / v_{z}=\tan (\theta)$, then the difference between the longitudinal kinetic energy $E_{z}$ and the total kinetic energy $E_{k}$, $\Delta E$, is given by

$$
\Delta E=E_{k} \sin ^{2}(\theta)
$$

Since $\theta$ remains constant under the above scaling rules as $E_{k}$ increases, it follows that the resolution of the electrostatic energy analyzer, $\Delta E$, is proportional to $E_{k}$. In another words, if we define the relative resolution $\Delta E / E$, then it is a constant independent of the total beam energy.

\section{BEAM TEST OF THE ENERGY ANALYZER}

The experimental setup, as shown in Fig. 6, consists of a gridded thermionic electron gun, three solenoidal magnetic lenses, and a diagnostic chamber. The energy analyzer is in the diagnostic chamber located between the second and the third solenoids. The third solenoid is after the energy analyzer and consequently, it has no effect for the beam trajectories in the energy analyzer. The distances of the first two solenoids and the energy analyzer from the electron gun are $9.1,21.6$, and $33.2 \mathrm{~cm}$, respectively. The solenoidal magnetic field extends less than $10 \mathrm{~cm}$ from the center, so there is practically no magnetic field inside the energy analyzer. Figure 7 depicts a typical beam envelope in the transport line calculated from the K-V envelope equation. We can see that the energy analyzer is located at the waist of the beam. The energy analyzer can be aligned with respect to the beam by a linear and rotation feedthrough.

Figure 8 is a typical output signal from the energy analyzer for a beam with an energy of $5 \mathrm{keV}$ and a pulse length of 100 ns. From the signal, we can see that this energy analyzer has a very fast rise time ( $\sim 2 \mathrm{~ns})$ and can reproduce the beam waveform faithfully. This is essential because some of the experiments conducted at the University of Maryland require time resolved energy spread from the beam. The way to measure the energy spread of the beam is to adjust the retarding voltage. As the retarding voltage is increased, the energy analyzer output becomes smaller. By differentiating the energy analyzer output voltage with respect to the retarding voltage, we can get the beam energy profile information. Figures 9 and 10 show such a case. In Fig. 9, different traces corresponding to different retarding voltages are plotted together. The numbers on the traces correspond to increasing retarding voltages. Note that the beam energy spread information is time resolved. We can see that, at the front end of the beam, the particle energy is larger than that in the main beam due to the longitudinal space-charge force. From this figure, we can construct the beam energy profile by taking the difference of the voltage of traces corresponding to the different retarding voltages. To study the energy spread of the main beam particles, we set the sampling point at the middle of the beam pulse. Figure 10 shows the energy profile. The 


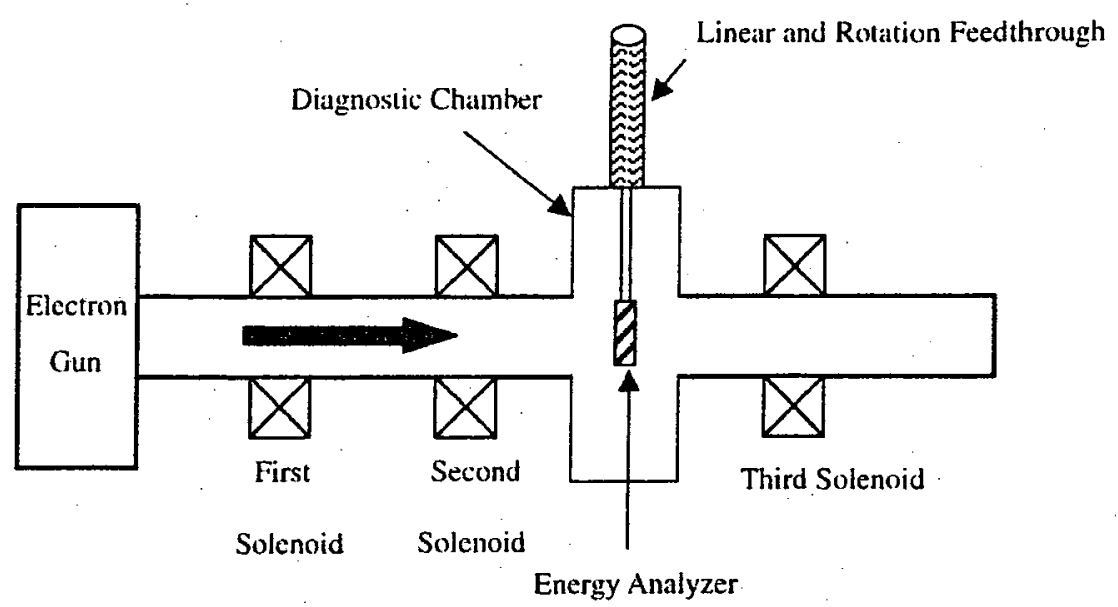

FIG. 6. Experimental setup for the energy analyzer test.

experimental data points and their fit curve are plotted together in this figure. The error bars are also shown here. The magnitudes of the error bars are determined by the signal fluctuations due to the background noise. Note that the energy distribution shown here is asymmetric and has a tail at the high-energy side. More study is underway to explain the source of this tail. In this experiment, the beam energy and current are $2.5 \mathrm{keV}$ and $60 \mathrm{~mA}$, respectively, which is strong space charge dominated. Because of the focusing forces introduced by the cylindrical structure, the defocusing forces due to the space-charge forces and other factors have been well balanced. The measured beam rms energy spread is $1.8 \mathrm{eV}$.

It is interesting to compare the experimental results with the theoretical predictions of the electron beam energy spread. In our example, the beam energy is $2.5 \mathrm{keV}$. The beam is emitted from a cathode with a radius of $5 \mathrm{~mm}$. In the transport system, the average beam radius is around $9 \mathrm{~mm}$. The first term of Eq. (6) gives the energy spread due to the longitudinal-longitudinal effect, which is $0.9 \mathrm{eV}$ in this case. The second term of Eq. (6) gives the rms energy spread due to the Boersch effect, which is $\sim 1.0 \mathrm{eV}$. The

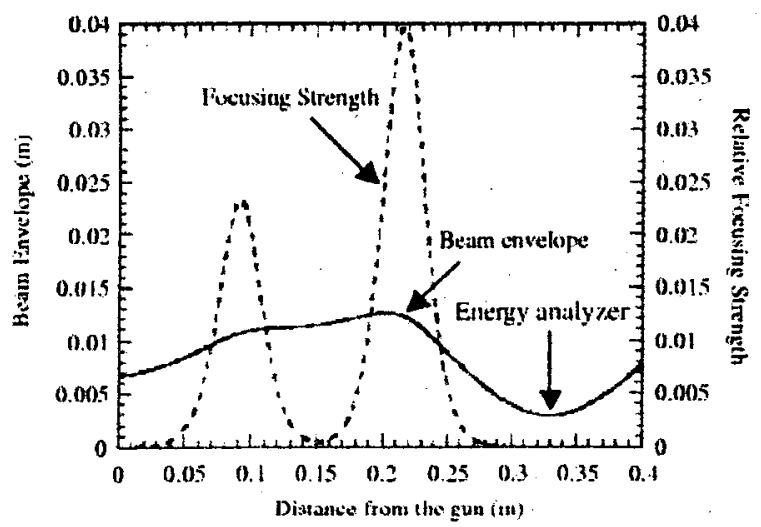

FIG. 7. (Color) Typical beam envelope in the transport line. final energy spread resulting from both effects as given by Eq. (6) is $\sim 1.3 \mathrm{eV}$, which is very close to the experimental result, $1.8 \mathrm{eV}$.

From the comparison, we find that the experimental result is larger than the theoretical predictions. There are several possible reasons for this discrepancy. First, as we explained earlier, the finite resolution of the energy analyzer will contribute to the larger measured beam energy spread. The computer simulation shows that the resolution of this energy analyzer is $0.6 \mathrm{eV}$ for a $2.5 \mathrm{keV}$ beam. In reality, because of the collective space-charge forces and Coulomb scattering of electron beams inside the energy analyzer, the resolution of the energy analyzer might be worse than the computer simulation. A two-dimensional particle-in-cell code simulation is underway to study the behavior of the energy analyzer in more detail. Second, besides these two main energy spread sources mentioned here, there are possible other sources, which may cause an increase of the beam energy spread. In the study reported here, we have considered only the Coulomb scattering theory. However, in space-charge-dominated beams,

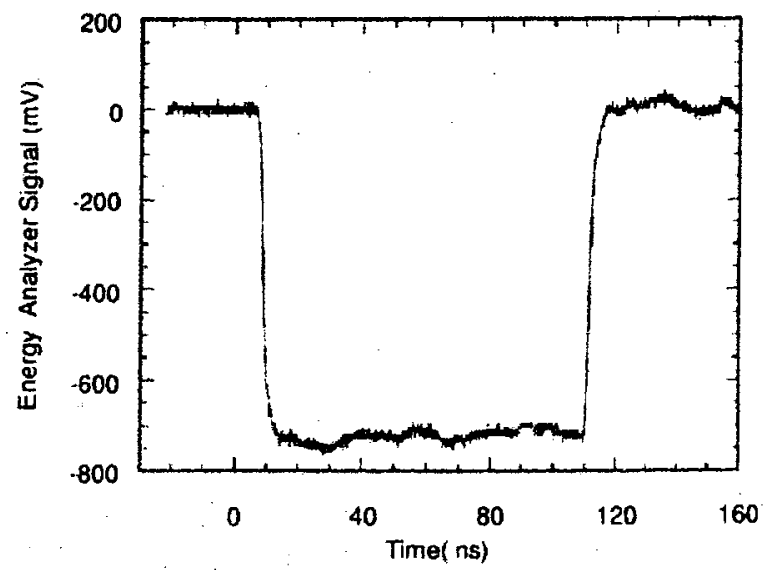

FIG. 8. (Color) Typical energy analyzer output signal. 


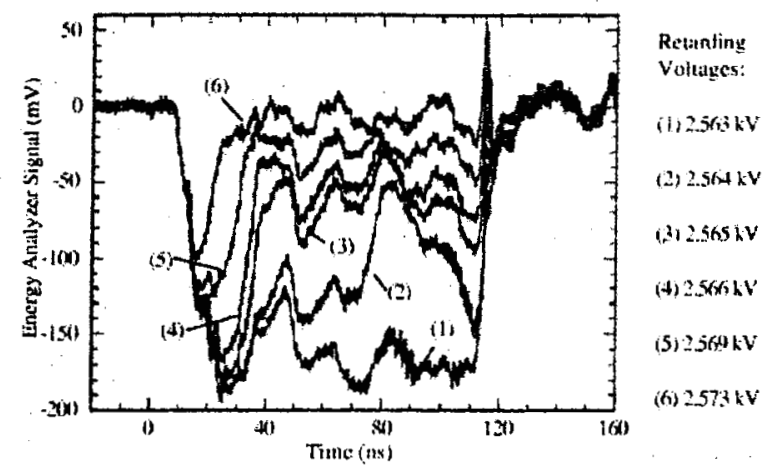

FIG. 9. (Color) Energy analyzer outputs at different retarding voltages.

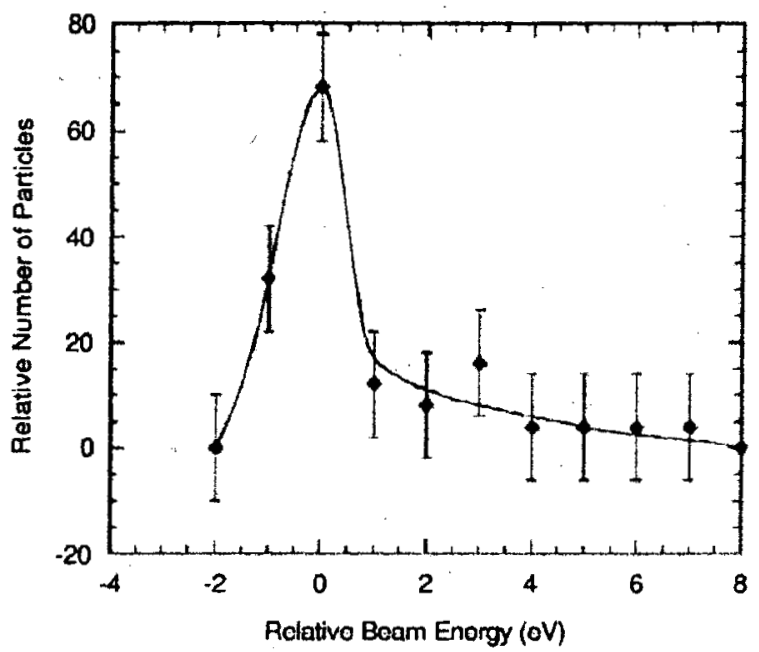

FIG. 10. (Color) Beam energy profile for a beam with energy of $2.5 \mathrm{keV}$ and current of $60 \mathrm{~mA}$. The rms energy width is $1.8 \mathrm{eV}$.

collective instabilities may produce relaxation towards 3D thermal equilibrium. The larger energy spread observed in the experiments may in fact be due to collective instabilities and other effects that will have to be studied in future simulation work. Actually, there are already some simulations in the literature, showing that a beam with a two-temperature anisotropy $\left(T_{\| i} \ll T_{\perp, i}\right)$ will develop instability, which may increase the longitudinal energy spread and even significantly deteriorate the beam quality [9-11].

\section{CONCLUDING REMARKS}

A compact high-resolution electrostatic energy analyzer for low-energy electron beams has been designed, simulated, and beam tested. The simulations and beam tests show that the energy analyzer has very good resolution for the energy measurement of space-charge-dominated electron beams in the energy range of several thousand electron volts. The measured beam energy spread is very close to the predictions of a theoretical model based on a longitudinal beam cooling effect, transverse-longitudinal, and longitudinal-longitudinal temperature relaxation effects. A new version of this analyzer, which has higher resolution for the $10 \mathrm{keV}$ UMER beam has been constructed and is currently being tested [12].

Last, it should be noted that our electrostatic analyzer is applicable only to low-energy electron and ion beams. At higher energies, other techniques have to be used to measure the energy spread of the beams.

\section{ACKNOWLEDGMENTS}

This work is supported by DOE Grants No. DEFG0292ER54178 and No. DEFG0294ER40855.

[1] J.G. Wang, H. Suk, and M. Reiser, Phys. Rev. Lett.79, 1042 (1997).

[2] P. G. O'Shea, M. Reiser, R. A. Kishek, S. Bernal, H. Li, M. Pruessner, V. Yun, Y. Cui, W. Zhang, Y. Zou, T. Godlove, D. Kehne, P. Haldemann, and I. Haber, Nucl. Instrum. Methods Phys. Res., Sect. A 464, 646 (2001).

[3] H. Boersch, Z. Phys. 139, 115 (1954).

[4] A. V. Aleksandrov et al., Phys. Rev. A 46, 6628 (1992).

[5] M. Reiser, Theory and Design of Charged Particle Beams (Wiley, New York, 1994).

[6] J. A. Simpson, Rev. Sci. Instrum. 32, 1283 (1961).

[7] H. Suk, Ph.D. Thesis, University of Maryland, 1996.

[8] Y. Zou, Ph.D. thesis, University of Maryland, 2000.

[9] A. Friedman, D. P. Grote, and I. Haber, Phys. Fluids B4, 2203 (1992).

[10] I. Haber, D. A. Callahan, A. Friedman, D. P. Grote, and A. B. Langdon, Fusion Eng. Des.32, 169-174 (1996).

[11] I. Haber, D. A. Callahan, A. Friedman, D. P. Grote, and S. M. Lund, Nucl. Instrum. Methods Phys. Res., Sect. A 415, 405 (1998).

[12] Y. Cui, A. Valfells, S. Bernal, M. Reiser, P. G. OShea, R. A. Kishek, V. Yun, and N. Rahimi, inProceedings of the 2001 Particle Accelerator Conference, Chicago, IL,edited by Peter W. Lucas and Sara Webber (IEEE, Piscataway, NJ, 2001). 


\title{
Simulation studies on matching of space-charge-dominated beams for the University of Maryland Electron Ring (UMER)
}

\author{
H. Li ${ }^{\dagger}$, S. Bernal, R.A. Kishek, I. Haber, Y. Zou, P.G. O'Shea and M. Reiser \\ Department of Electrical and Computer Engineering, Institute for Research in Electronics and Applied Physics, \\ University of Maryland, College Park, MD 20742
}

\begin{abstract}
The physics of space-charge-dominated beams with very large tune shift has never been explored in existing machines. The University of Maryland Electron Ring (UMER), currently under construction, will study the nonlinear physics characteristics of intense space-charge-dominated beams on a scaled basis. The unique features of UMER challenge us to design the beam optics carefully. One of the most important issues is to match the beam from the electron gun through the injector into the ring lattice. This is the major topic of this paper. The physics background, matrix and linear envelope-code computations, and particlein-cell simulations of beam transport over a long propagation distance in UMER are presented.
\end{abstract}

\section{Introduction}

The physics of space-charge-dominated beams with very large tune shift has never been explored in existing machines, where space-charge effects and tune shifts are relatively small. In advanced accelerators with high-intensity beams, however, such as spallation neutron sources, heavy-ion fusion drivers, and intense light sources, space-charge effects will play a very significant role in the beam physics and machine performance. The University of Maryland Electron Ring (UMER) [1,2], currently under construction, is designed as a tool to study the behavior of intense, space-charge-dominated beams on a scaled basis. By using a low-energy $(10 \mathrm{keV})$, high-current $(100 \mathrm{~mA})$ electron beam in a compact ring of circumference $11.52 \mathrm{~m}$, UMER represents a low-cost laboratory facility for scaled experiments, computer-code development and validation, and testing of theoretical models. The main focusing lattice of UMER is composed of 36 FODO periods, each of which consists of two printed-circuit (PC) quadrupoles and a PC dipole [3], with a period length of only $32 \mathrm{~cm}$. The stringent space requirement dictates the need for short printed-circuit quadrupoles and dipoles, which not only meets the special field quality requirements, but also offers great flexibilities in the field strength adjustment. The strong space-charge-dominated beams and compact features of UMER have challenged us to design the beam optics carefully. Although previous general studies of single-particle dynamics in UMER have been reported [4], we present here an update covering both single-particle and space-charge beam dynamics. Furthermore, we extend the computer simulations reported in connection to experiments in a prototype matching section [5]. In the paper, we organize our studies in the following order: beam physics background, beam matching studies, collective beam physics effects and summary.

\section{Beam physics background}

It is well known that the transport of intense beams can be approximately modeled by theKapchinskyVladimirsky (KV) [6] distribution. An equivalent KV distribution can be defined for any beam regardless of its spatial profile, as long as the two distributions have the samerms radius and emittance [7]. In UMER, the beam bunch $(10 \mathrm{keV}, 100 \mathrm{~ns})$ has a radius in the range of $10 \mathrm{~mm}$ or less depending on the beam current and focusing. Since the bunch's length is about $6 \mathrm{~m}$, the beam can be considered as a 2D beam, except for the edge effects. Thus, the beam transport is governed by the 2D KV envelope equations:

$$
X^{\prime \prime}+\kappa_{x} X-\frac{2 K}{X+Y}-\frac{\varepsilon_{x}^{2}}{X^{3}}=0
$$

\footnotetext{
${ }^{\dagger}$ E-mail: lihui(a)eng.umd.edu
} 


$$
Y^{\prime \prime}+\kappa_{y} Y-\frac{2 K}{X+Y}-\frac{\varepsilon_{y}^{2}}{Y^{3}}=0
$$

$X$ and $Y$ are the $2 \times$ rms beam radii in the two planes perpendicular to the propagation direction, and $X^{\prime \prime}$ and $Y^{n}$ represent the change of slopes of $X$ and $Y$ along the focusing channel. Further, $K=\left(I / I_{0}\right) \cdot\left(2 / \beta^{3} \gamma^{3}\right)$ is the generalized dimensionless perveance with the characteristic current $I_{0}=4 \pi \varepsilon_{0} m c^{3} / q \approx 17 \mathrm{kA}$ for electrons [7], and $\kappa_{x}(s)$ and $\kappa_{y}(s)$ are the external focusing functions. The last terms in Eqs. (1a) and (lb) contain the effective ( $4 \times \mathrm{rms}$, unnormalized) emittances $\varepsilon_{\mathrm{x}}$ and $\varepsilon_{\mathrm{y}}$.

To understand the beam physics, we can use the smooth approximation for the matched beam with a constant beam radius $\bar{X}=\bar{Y}=a$ in a uniform focusing channel. In this approximation,

$$
k_{0}^{2} a-\frac{K}{a}-\frac{\varepsilon^{2}}{a^{3}}=k^{2} a-\frac{\varepsilon^{2}}{a^{3}}=0
$$

where $k_{0}=2 \pi / \lambda_{0}$ is the betatron wavenumber without space charge and $\lambda_{0}$ is the betatron wavelength; $k=k_{0}\left(1-K / k_{0}^{2} a^{2}\right)^{1 / 2}=2 \pi / \lambda$ is the betatron wavenumber with space charge. $k_{0}^{2} a$ represents the average external focusing force that balances the space-charge force and the emittance force. The ratio of spacecharge force to external focusing force can be expressed by the dimensionless intensity parameter $\chi=K / k_{0}^{2} a^{2}$ [1]. In terms of $\chi$, the betatron tune depression is given by $k / k_{0}=(1-\chi)^{1 / 2}$. If $0.5<\chi<1$, the beam is space-charge-dominated. If, on the other hand, $0<\chi<0.5$, the beam is in the emittancedominated regime, in which most existing machines are operating. By varying the electron beam voltage and current, UMER can operate in a wide regime, with $\chi$ ranging from 0.2 to about 0.97 . In the highly space-charge-dominated regime $(\chi=0.97)$, the tune depression $\mathrm{k} / \mathrm{k}_{0}$ is about 0.16 , which corresponds to an electron beam of $100 \mathrm{~mA}, 10 \mathrm{keV}, 60 \mu \mathrm{m}$ effective emittance and zero-current phase advance per period $\sigma_{0}=76^{\circ}$, where $\sigma_{0}=k_{0} S=\left(2 \pi / \lambda_{0}\right) S$, and $S=0.32 \mathrm{~m}$ is the length of one focusing period. Successful beam transport in this new regime demands accurate matching calculations.

\section{Beam matching studies}

The layout of UMER is shown in Figure 1 [2]. Its main lattice consists of three parts: injector, ring, and extractor. A detailed description of UMER can be found in our website [8] or other papers [1,2]. In this section, we will focus on the optics design of the ring lattice and the injector.

UMER consists of a series of magnetic quadrupoles, with strength $\kappa_{x}=-\kappa_{y}=q G / m \gamma \beta c$, where $G=G(s)$ is the quadrupole field gradient. In practice, it is common to model the quadrupole by a "hardedge" element with an effective length defined by $l_{\text {eff }}=1 / \kappa_{\text {peak }} \cdot \int_{-\infty}^{+\infty} \kappa(z, r=0) d z$. Typical smooth and "hard-edge" gradient profiles of a unit FODO cell are shown in Figure 2. We normally use "hard-edge" magnets in KV envelope and matrix codes to do first-order matching calculations. The advantages of "hard-edge" magnets reside on their easy implementation in the codes, because the fields of adjacent magnets do not normally overlap. However, a more realistic calculation can be done with accurate 3D fields for the magnets in a particle-in-cell (PIC) code. To generate these fields along the beam line, a new air-core magnetic field solver, MAGLI [9], was developed. The code can model the printed-circuit magnets by up to 18 million conductor segments in a one-gigabyte memory space.

\section{Ring lattice design}

We illustrate now three different models for calculating the peak focusing strengths of quadrupoles in a unit FODO cell in UMER for $\sigma_{0 x}=\sigma_{0 y}=76^{\circ}$. The results are summarized in Table $I$. In the first model, 
"hard-edge" quadrupoles in a straight section are used. In this case, a simple matrix calculation using the standard matrices for quadrupoles and drifts yields $\kappa_{x 1}=-\kappa_{x 2}=-230 \mathrm{~m}^{-2}(7.8 \mathrm{G} / \mathrm{cm})$. If smooth gradient profiles in a straight section are employed, the matrix approach is still possible if the profiles are modeled by the superposition of a number of "hard-edge" sub-elements. The results for this case (not shown in Table I) are $\kappa_{x 1}=-\kappa_{x 2}=-234 \mathrm{~m}^{-2}$. In the second model, the small focusing of the bending dipoles is taken into account. The UMER bending dipole is equivalent to a sector dipole with a field index $\mathrm{n} \approx 0.72$, where $n=-\left\{r_{0} / B_{y \text {-int }}(x=0)\right\} \cdot\left\{\partial B_{y \text {-int }}(x) / \partial x\right\}, r_{0}$ is the equilibrium radius. The dipole focusing can be expressed as $\kappa_{x}=\left(\alpha^{2} / l_{d}{ }^{2}\right) \cdot(1-n), \kappa_{y}=\left(\alpha^{2} / l_{d}{ }^{2}\right) \cdot n$. Here $\alpha=10^{\circ}$ is the bending angle and $l_{d} \approx 3.86 \mathrm{~cm}$ is the dipole's effective length along the bending path. Since $0<n<1$, the dipole induces focusing in both the horizontal $(x)$ and the vertical $(y)$ directions, thus increasing the net focusing in a FODO period by a small amount.

Finally, Model III employs the WARP code [10] to track the centroid of thousands of particles (without space charge) for several turns through the 3D fields of the UMER magnets. All magnetic fields are precalculated along the beam line in $1 \mathrm{~mm} \times 1 \mathrm{~mm} \times 1 \mathrm{~mm}$ grids with high accuracy by MAGLI. The average betatron-tune is determined by an interpolated-FFT technique [11], and the desired $\sigma_{0}=76^{\circ}$ is achieved by varying the real quadrupole strengths iteratively by a Newton method.

$\begin{aligned} & \text { Table 1. Peak quadrupole strengths in a FODO period from different lattice models, } \\
& \text { for } \sigma_{0 \mathrm{x}}=\sigma_{0 \mathrm{x}}=76^{0} \text {. }\end{aligned}$
\begin{tabular}{lcc} 
& & \\
\hline & -230 & 230 \\
\hline I. Hard-edge quads (Matrix) & -220 & 224 \\
II. Hard-edge quads + Dipole ( $=0.72$, Matrix) & -222 & 227 \\
III. Real quads + real dipole (WARP code) & $\kappa_{\mathrm{x} 2}\left(\mathrm{~m}^{-2}\right)$ \\
\hline
\end{tabular}

As seen from Table I, model II is fairly realistic. However, the same model with $\mathrm{n}=0$ would yield $\kappa_{\mathrm{x} 1} \approx-227 \mathrm{~m}^{-2}, \kappa_{\mathrm{x} 2} \approx 217 \mathrm{~m}^{-2}$.

Models I and II can be easily implemented in an envelope code to achieve periodic envelope matching in a FODO cell. However, it is instructive to compare the results of using a PIC code with the full 3D fields for both quadrupoles and dipoles with the quadrupole peak strengths taken from Table I. Figures 3(a) to 3(c) show the results of such simulations with the WARP code. Fig. 3(a) shows a periodic solution, but also reveals a $10 \%$ difference between $X$ and $Y$ envelope peaks. This is clearly due to the unequal $\sigma_{\mathrm{x} 0}, \sigma_{\mathrm{y} 0}$. Figure 3(b) and Fig. 3(c), on the other hand, show the $X, Y$ envelopes by employing quadrupole strengths from models II and III, respectively. A closer view of the different envelopes demonstrates that model-II can be used in practice.

\section{Matching section design}

In designing the matching section (injector), we aim to inject the beam into the ring with the desired beam radii and slopes $\left(X_{R 0}, Y_{R 0}, X_{R 0^{\prime}}, Y_{R 0^{\prime}}\right)$, as dictated by the results of periodic FODO matching (previous section). The UMER injector has seven adjustable parameters (the strengths of one solenoid and six quadrupoles, $Q_{1}$ to $Q_{6}$ ) to reach this goal. The "hard-edge" focusing function of the injector is depicted in Figure 4. While the magnets locations are fixed, their strengths can be adjusted. Initial matching calculations are done by integrating the $\mathrm{KV}$ envelope equations and iteratively adjusting the peak strengths of all seven elements. The initial conditions $\left(X_{0}, Y_{0}, X_{0}, Y_{0}\right)$ are specified at the plane of an aperture plate near the electron gun output. From experiment, we know that this plane is very close to the beam waist, so $X_{0}^{\prime} \approx Y_{0}^{\prime} \approx 0$. If we define $\left(X_{1}(\bar{\kappa}), Y_{1}(\bar{\kappa}), X_{1}^{\prime}(\bar{\kappa}), Y_{1}^{\prime}(\bar{\kappa})\right)$ as the beam radii and slopes after integrating Eqn. (1) 
to the injection point, where $\bar{\kappa}=\left(\kappa_{0}, \kappa_{1}, \kappa_{2}, \kappa_{3}, \kappa_{4}, \kappa_{5}, \kappa_{6}\right) \in R^{7}$ are the strengths of the solenoid and the quadrupoles $\mathrm{Q}_{1}$ to $\mathrm{Q}_{6}$, the matching error can be expressed as $f(\bar{\kappa})=\left(X_{1}-X_{R 0}, Y_{1}-Y_{R 0}, X_{1}^{\prime}-X_{R 0}^{\prime}, Y_{1}^{\prime}-Y_{R 0}^{\prime}\right)$. By minimizing $\|f(\bar{\kappa})\| \rightarrow 0$, the solution space $\left\{\bar{\kappa}^{*}\right\}$, a subset of $R^{7}$, can be obtained. Since there are infinite solutions in $\left\{\bar{\kappa}^{*}\right\}$, we may choose the optimal one according to some specific criteria. The optimization can be done as stated using, for example, the optimization toolbox of MATLAB [12]. Alternatively, a solution can be found in two steps by first optimizing the "distance" from the envelopes to a properly chosen "reference" trajectory (normally defined by the average beam radius in the periodic lattice) and then minimizing $\|f(\bar{\kappa})\|$ as before. This latter approach is used in the envelope code SPOT [13]. In practice, avoiding large envelope excursions is the natural consideration in order to reduce the effects of lens nonlinearities and possible image forces from beam offsets. The optimization problem that minimizes large excursions can be stated as follows.

$$
\underset{\left.\mid \bar{\kappa}^{*}\right\}}{\operatorname{Minimax}}\left(X\left(\bar{\kappa}^{*}, z\right) \& Y\left(\bar{\kappa}^{*}, z\right)\right)
$$

Finding an optimal solution $\bar{\kappa}^{*}$ would take very significant computing power because of the double optimization required. To our knowledge, no algorithm exists that can generate a global minimum for this problem. In spite of this, we are able to reach some local optima with constraints. Although the local optimal solution depends on the initial guess of $\bar{\kappa}^{*}$, it is still acceptable if a specified constraint is satisfied, e.g. the largest envelope excursion is smaller than an allowable value.

Finally, the solution from MATLAB needs to be refined with PIC simulations including realistic magnets. Typically we use $320 \mathrm{k}$ particles, $256 \times 256$ grids for the Poisson solver, $1 \mathrm{~mm} \times 1 \mathrm{~mm}$ grid-size for the pre-calculated fields along the path and $2 \mathrm{~mm}$ step-size for the particle-pushing. Figure 5 illustrates the beam cross-section pictures along the injector and the ring. The pictures in the first three columns correspond to the injector from Q1 to Q6; each of the following columns shows a snapshot in a period length of exactly $32 \mathrm{~cm}$. The first and third rows show simulated pictures at the center plane of each quadrupole, while the second and fourth rows correspond to pictures at planes halfway between quadrupoles. The initial particle distribution is Semi-Gaussian (SG), i.e. a distribution with uniform particle density in space but a Gaussian profile in the transverse velocity. Because the matching is done with a KV beam and assuming no emittance changes, it is not surprising to observe some mismatches downstream for a SG beam. In our simulation, a few percent of mismatch is acceptable for transportation over several turns.

\section{Collective beam physics effects}

In UMER, the average radius of the full beam $(100 \mathrm{~mA}, 10 \mathrm{keV}, 60 \mu \mathrm{m})$ for $\sigma_{0}=76^{\circ}$ is about $a \approx 1 \mathrm{~cm}$. Further, the beam pipe radius is $b \approx 2.5 \mathrm{~cm}$. Thus, image charges induced by an offset beam will affect the beam so the betatron tune will change [7]. For a small beam centroid offset, the shift will be from $v_{0}=7.6$ to $\left(1-a^{2} / b^{2}\right)^{1 / 2} \cdot v_{0} \approx 7.0$. Simulations with WARP where both space charge and beam-tube boundaries are included agree well with this theoretical prediction. Since the effective tune is independent of beam misalignment (as long as the misalignment is relatively small compared to the pipe radius), measurement of the actual tune in an experiment will provide a definite test of this effect.

Another collective effect relates to emittance growth observed in simulations. For a beam starting with a $\mathrm{KV}$ distribution, there is no emittance growth observed for several turns if we disregard the longitudinal energy spread in the simulation. However for a SG beam, small emittance growth has been observed in the injector region while it does not happen in the ring. Similar observations have been reported in the numerical studies of the LBNL Heavy-Ion Beam Combiner Experiment [14]. We believe that the emittance growth is related to the large ellipticity of the beam cross section at the middle planes of quadrupoles Q1 and Q2 of the injector, as can be seen in the first two columns of Fig. 5. This illustrates the importance of the "Minimax" optimization problem in the matching section design. 
Finally, we discuss briefly beam halo formation due to beam mismatch. The phenomenon has been reported very recently in connection to proton beam experiments [15]. It is of great practical interest not only because it is related to beam degradation and emittance growth, but also to the possibility of pipe activation. We have observed halos in UMER experiments with a $10 \mathrm{keV}, 25 \mathrm{~mA}$ beam, but none with the full beam (at $10 \mathrm{keV}, 100 \mathrm{~mA}$, see S. Bernal et al in these Proceedings). While these are preliminary results, WARP simulations with the full beam and a $10 \%$ mismatch of the initial envelope do not show clear halo formation over two turns. Additional experiments are planned that take advantage of the flexibility of the UMER source and lattice to vary the initial beam distribution as well as the mismatch. Simulations with the apertured, $25 \mathrm{~mA}$ beam are in progress.

\section{Summary}

We have reviewed in this paper the basic physics of continuous (2D) space-charge-dominated beams, and presented beam matching studies for the UMER facility. We have employed both simple KV envelope codes and complex WARP-PIC simulations to aid us in designing the beam optics. A dipole model with field index $n=0.72$ has been developed to include bending in the envelope codes. It is also indispensable to use PIC simulations with real fields and bends to refine the matching section design in order to minimize emittance growth. Finally, image-charge effects on the betatron tune have been simulated and compared with the theory.

\section{Acknowledgement}

This work is supported by the U.S. Department of Energy.

\section{References}

[1] M. Reiser et al., Proceedings of the 1999 IEEE Particle Accelerator Conference, p. 234 (2000).

[2] P.G. O'Shea et al., The University of Maryland Electron Ring (UMER), Nucl. Instr. and Meth. A 464, p. 646-652 (2001).

[3] W.W. Zhang et al., Design and Field measurement of Printed-Circuit Quadrupoles and Dipoles, Phys. Rev. ST Accel. Beams 3, 122401 (2000).

[4] M. Venturini, Ph.D. Thesis, Dept. of Physics, University of Maryland, College Park, 1998; M. Venturini et al, Fusion Eng. Des. 32-33, 283 (1996).

[5] S. Berna et al, Phys. Rev. ST Accel. Beams I, 044202 (1998).

[6] I.M. Kapchinsky, Theory of Resonance Linear Accelerators, Harford Academic Press, New York, 1985.

[7] M. Reiser, Theory and Design of Charged Particle Beams, Wiley, New York, 1994.

[8] UMER website: http://www.ireap.umd.edu/umer.

[9] H. Li, MAGLI Manual for Windows and Linux (2002).

[10] D.P. Grote, et. al, Fus. Eng. \& Des. 32-33, 193-200 (1996).

[11] E. Asseo, CERN PS/85-3, LEA (1985).

[12] The Mathworks, Inc, 1984.

[13] C.K. Allen, et. al, Optimal Transport of Low Energy Particle Beams, Proceedings of the 1995 IEEE Particle Accelerator Conference, p. 2324 (1996).

[14] W.M. Fawley et al, Numerical Simulation Studies of The LBNL Heavy-Ion Beam Combiner Experiment, Proceedings of the 1997 IEEE Particle Accelerator Conference, p. 1923 (1998).

[15] C.K. Allen et al, Beam-Halo Measurements in High-Curent Proton Beams, Phys. Rev. Lett. 89, 214802 (2002). 


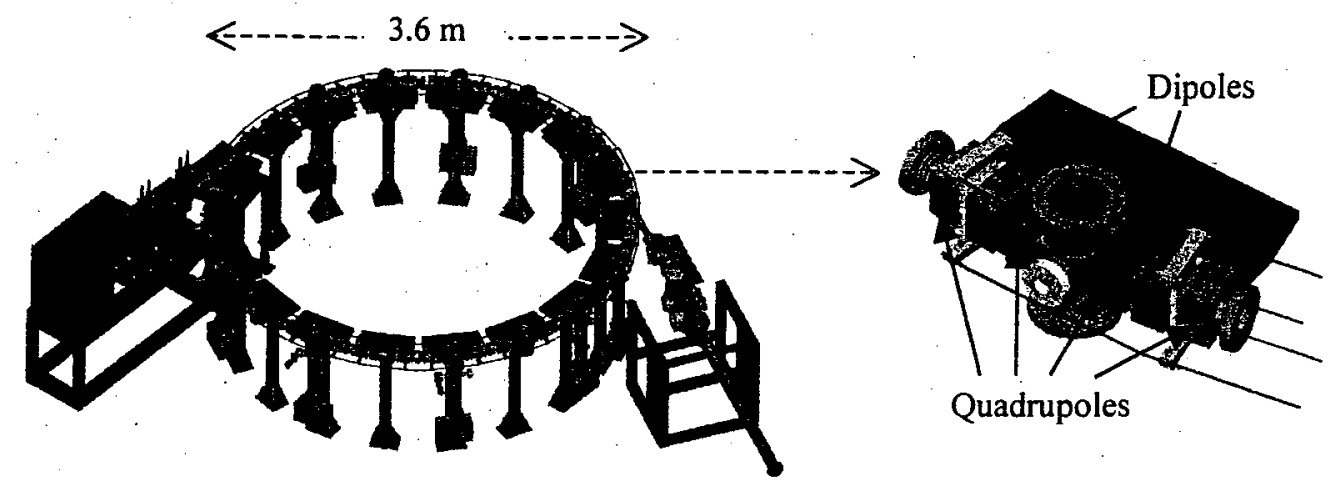

Fig. 1. UMER Layout and detailed structure of a $20^{\circ}$ bend (two FODO periods)

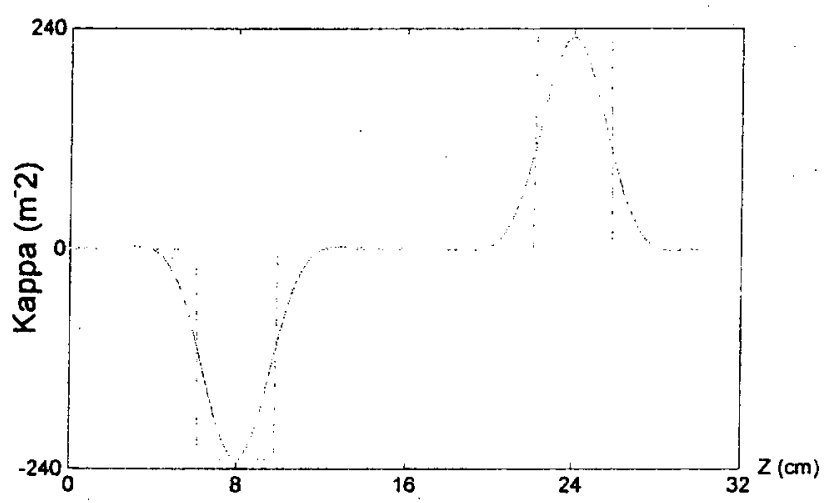

Fig. 2. Real quadrupole profile vs. "hard-edge" quadrupoles

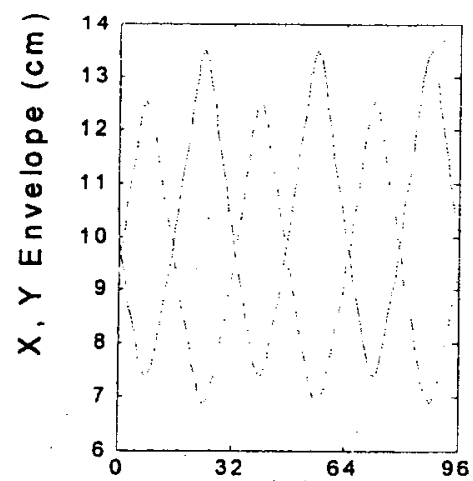

(a)

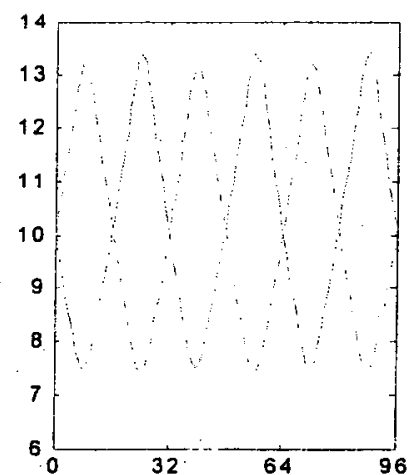

(b)

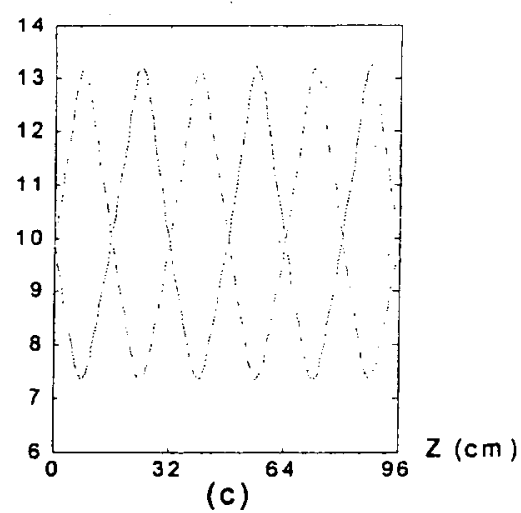

(c)

Fig. 3. $X, Y$ envelopes from WARP simulations (full 3D fields and bending) employing peak quadrupole focusing strengths from Table I: (a) quadrupole strengths from simplest model, (b) bending included, and (c) full model. Solid line: $X$-envelope; Dotted line: $Y$-envelope. 


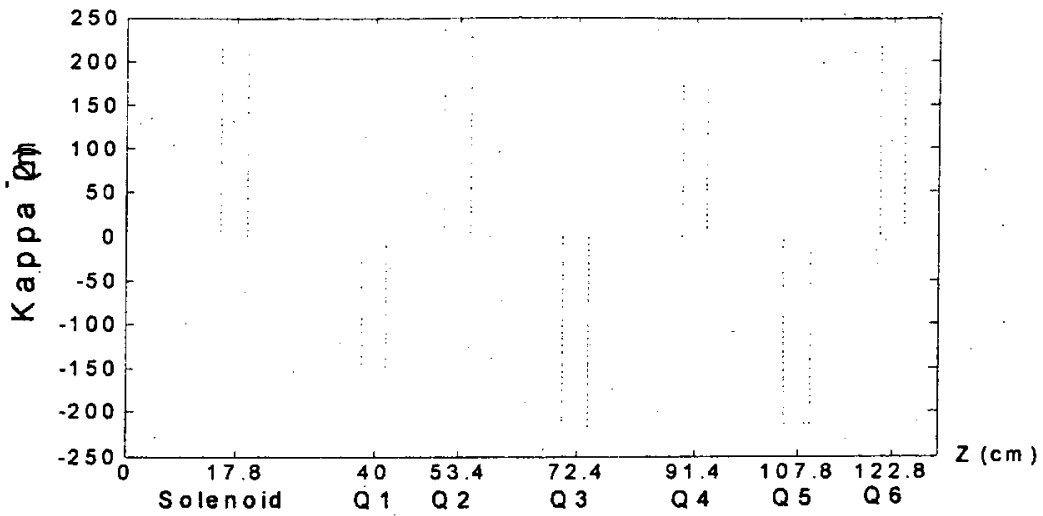

Fig. 4. Matching section model

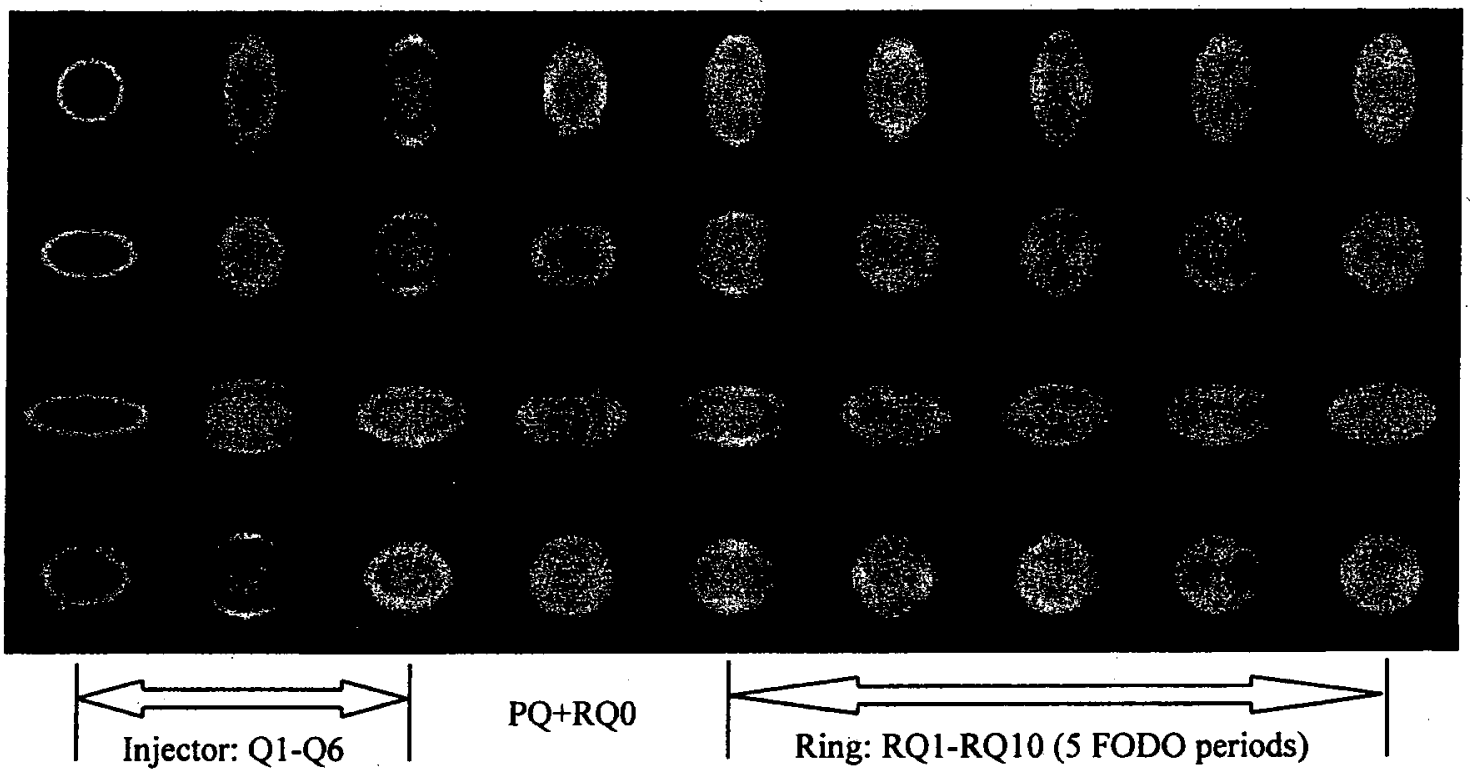

Fig. 5. WARP simulations of beam cross sections along the injector and five FODO periods in UMER. The electron beam parameters are: $10 \mathrm{keV}, 100 \mathrm{~mA}, 60 \mu \mathrm{m}$ (effective emittance), with $\sigma_{0 x}=\sigma_{0 y}=76^{\circ}$. 


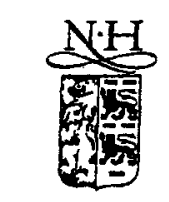

ELSEVIER

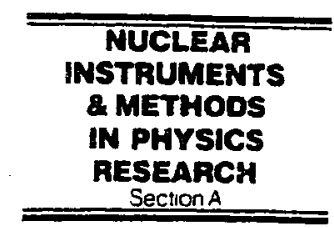

www.eisevier.nl/locate/nima

\title{
Injector for the University of Maryland Electron Ring (UMER)
}

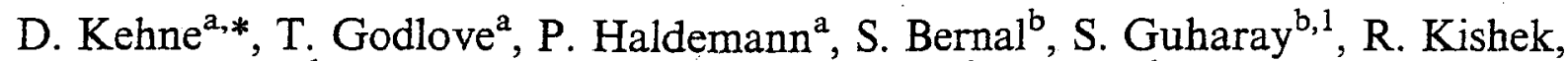 \\ Y. Li ${ }^{\mathrm{b}}$, P. O'Shea ${ }^{\mathrm{b}}$, M. Reiser ${ }^{\mathrm{b}}$, V. Yun ${ }^{\mathrm{b}}$, Y. Zou ${ }^{\mathrm{b}}$, I. Haber ${ }^{\mathrm{c}}$ \\ a FM Technologies, Inc., 10529 B Braddock Rd, Fairfax, VA 22032, USA \\ ' Institute for Plasma Research, University of Maryland, College Park, MD 20742, USA \\ ${ }^{\circ}$ Naval Research Laboratory, 4555 Overlook Drive SW, Washington, DC 20375, USA
}

\begin{abstract}
The electron beam injector constructed by FM technologies for the University of Maryland Electron Ring (UMER) program is described. The program will use an electron beam to model space-charge-dominated ion beams in a recirculating linac for heavy ion inertial fusion, as well as for high-current muon colliders. The injector consists of a $10 \mathrm{keV}, 100 \mathrm{~mA}$ electron gun with $50-100 \mathrm{nsec}$ pulse width and a repetition rate of $120 \mathrm{~Hz}$. The e-gun system includes a 6-mask, rotatable aperture plate, a Rogowski current monitor, an ion pump, and a gate valve. The injector beamline consists of a solenoid, a five-quadrupole matching section, two diagnostic chambers, and a fast current monitor. An independent diagnostic chamber also built for UMER will be used to measure horizontal and vertical emittance, current, energy, energy spread, and the evolution of the beam envelope and profile aiong the injector beamline. (C) 2001 Elsevier Science B.V. All rights reserved.
\end{abstract}

\section{Introduction}

The study of space-charge dominated beams is important for several major applications, including heavy ion inertial fusion and high-current muon colliders, as well as being the subject of general interest. Limits on beam current, emittance, propagation over distance and stability are not yet well understood in ring geometry. Spacecharge dominated beams are characterized by high

\footnotetext{
Work supported by the Small Business Innovation Research program of the US DOE.

*Corresponding author. FM Technologies, Inc., 10529-B Braddock Road, Patriot square, Fairfax, VA 22032-2236, USA. Tel.: + 1-703-941-0495; fax: +1-703-941-3624.

E-mail address: dkehne@gmu.edu (D. Kehne).

${ }^{1}$ Current address: FMI Technologies, Inc.
}

perveance and low transverse emittance, where generalized perveance is defined as $K=$ $2\left(I / I_{0}\right)(m / M)(\beta \gamma)^{-3} . \quad I$ is the beam current, $I_{0}=17 \mathrm{kA}, m$ and $M$ are the mass of an electron and particle in question, and $\beta$ and $\gamma$ are the normalized velocity and total energy of the particle. The FMT e-gun has $K=0.0015$, which has space-charge forces analogous, for example, to kiloamps of $\mathrm{GeV}$ heavy ions.

The purpose of the research effort described here is to develop an injector system, including the electron gun and injector line components for the University of Maryland Electron Ring (UMER) described elsewhere in this Conference [1]. In addition, a multi-purpose diagnostic chamber has been developed which is designed to characterize the beam from the e-gun, from the injector line, 
and finally from several positions around the ring as sections are added. Currently the e-gun and high-voltage deck are complete and tested, the diagnostic chamber is complete but not yet fully tested, and all of the remaining components have been designed and most of them are complete.

\section{Electron gun}

The design of the electron gun has been described [2]. For this purpose the well-known EGUN code was used [3]. The e-gun employs a commercial $8 \mathrm{~mm}$ diameter dispenser cathode with integral filament and grid. It includes a micrometer-controlled adjustable $A / K$ gap to vary the beam current, a gate valve and an ion pump. A built-in Rogowski-type current monitor is included, as is a rotatable calibrated aperture plate with six masks. The plate includes a pepperpot and a five-beamlet aperture as well as four round apertures ranging from $0.5 \%$ to $100 \%$ transmission. The e-gun can be baked to $250^{\circ} \mathrm{C}$. A photograph taken before final connections is shown in Fig. 1 taken final connections. The solenoid mount is to the right and the high voltage deck is visible on the leftside.

The cathode assembly is mounted within the vacuum system using a commercial coaxial feed-

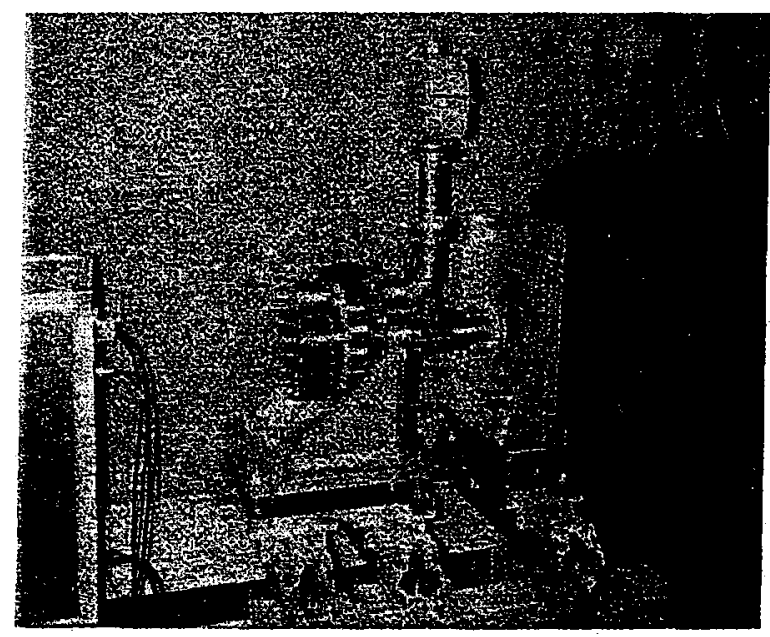

Fig. 1. UMER electron gun. through. The support structure is supplied with wheels for portability as well as a rigid mounting system for accurate location based on floor monuments. All of the appropriate elements are adjustable in six degrees of freedom.

\section{High-roltage deck}

The grid, cathode and grid pulser all float at $10 \mathrm{kV}$ on a conventional high voltage deck designed in collaboration with UMD. The deck houses a filament transformer, a grid bias supply, and a $180 \mathrm{~V}$ power supply. Pulses of $50-100 \mathrm{nsec}$ are generated using an internal pulse-forming cable which can be changed as desired. The fast switch, an NTE2347 transistor, can be operated in either an avalanche mode for a rectangular pulse, or at lower voltage to produce a parabolic pulse. Trigger pulses are supplied to the deck with a fiberoptic link and power via an isolation transformer. The deck is shown schematically in Fig. 2 and examples of beam pulses are shown in Fig. 3.

\section{Diagnostic chamber}

The multi-purpose vacuum chamber, shown in Figs. 4 and 5 , is based on an 8 in. diameter chamber with multiple ports. It is designed to measure horizontal and vertical emittance with a slit-wire technique and includes a retarding-field analyzer to measure energy and energy spread. The slits are mounted on commercially purchased MDC linear feedthroughs with 6 in. travel and mounted stepper motors. The position calibration of the feedthroughs is $0.32 \mu \mathrm{m} / \mathrm{step}$. The wire and energy analyzer linear feedthroughs were especially designed by FMT. They allow the required electrical signal to enter through the same port. In addition, they have greater lateral stability than the MDC feedthroughs. The position calibration of these feedthroughs is $0.64 \mu \mathrm{m} / \mathrm{step}$. LABVIEW software has been written for control and data acquisition. A movable phosphor screen with calibrated $1.5 \mathrm{~m}$ travel is included for insertion into the complete injector as well as the e-gun. A Faraday cup and pepperpot are also supplied. 


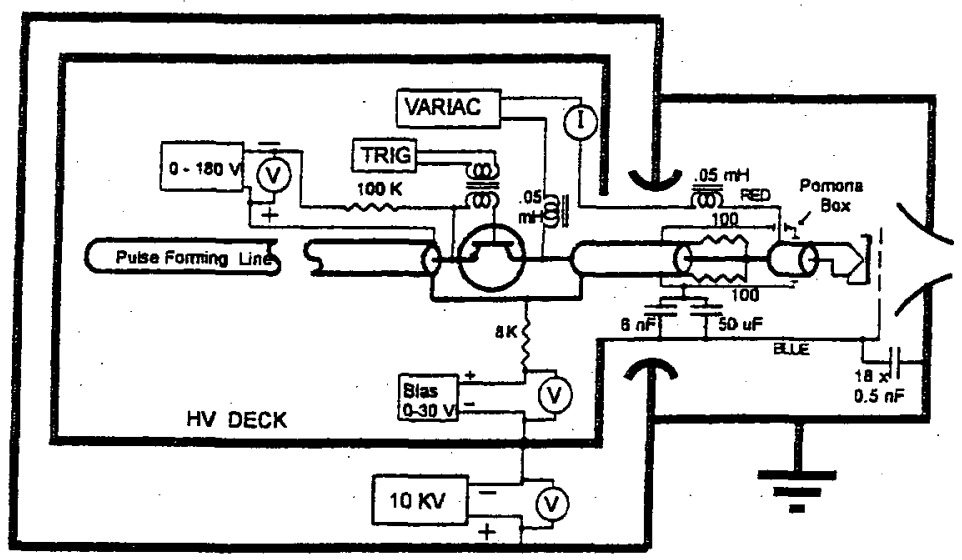

Fig. 2. Schematic of the high-voltage deck showing connections to the electron gun.

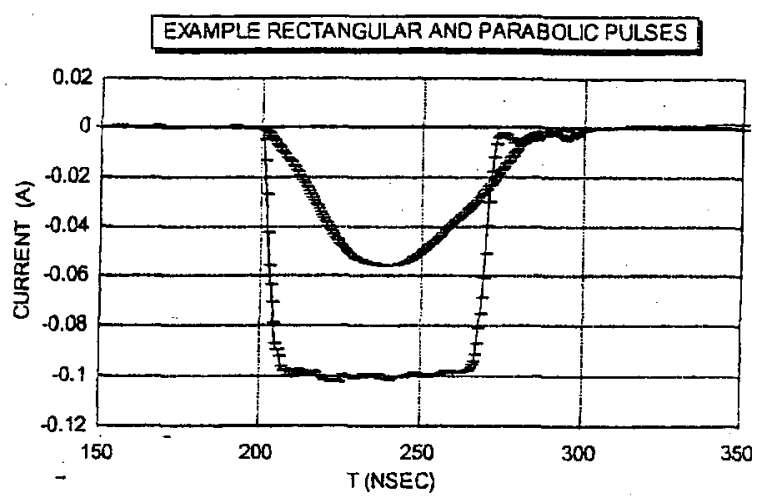

Fig. 3. Beam puises obtained during e-gun testing using a fast current transformer.

Viewing ports provide a visual check on device insertion.

\section{Injector beamline}

A scale drawing of the UMER injector, giving the location of major elements, is shown in Fig. 6. The Panofsky quadrupoles are pulsed sequentially, Panl for the injected beam and $\operatorname{Pan} 2$ for the ring beam. FM technologies is responsible for the injector system up to but not including the $\mathrm{Y}$ shaped injection chamber, with the exception of certain components supplied by UMD which are

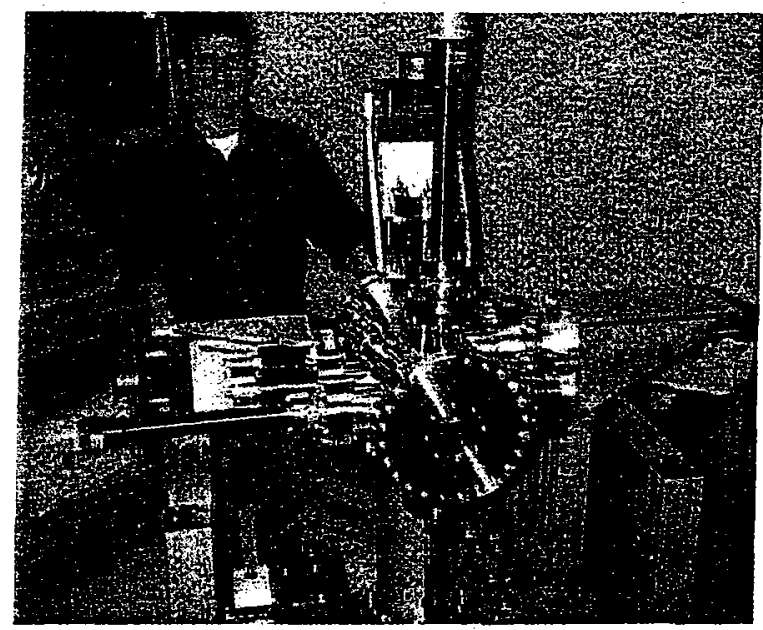

Fig. 4. Diagnostic chamber nearing completion.

natural to their program. These include the solenoid, printed-circuit quadrupoles, and the diagnostic devices to be inserted into the beam position monitor chambers. The detailed locations of the solenoid, the five quadrupoles, and the pulsed dipole and quadrupole are primarily based on beam-matching calculations [4].

Helmholtz conductors ( $\mathrm{HC}$ ) are planned for earth field compensation, two sets each for the horizontal and vertical planes. The magnetics design is complete. Four conductors, located in a $30^{\circ} / 60^{\circ}$ rectangular array at $11 \mathrm{~cm}$ radius, are sufficient to provide excellent field uniformity over 


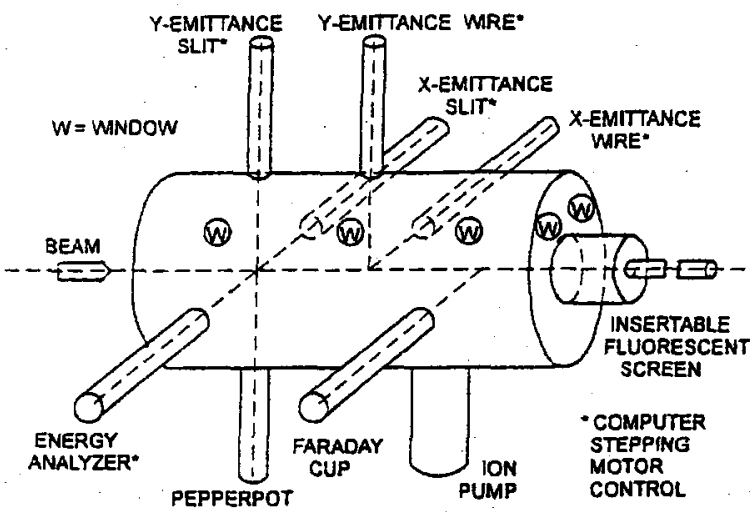

Fig. 5. Diagnostic chamber schematic. The fluorescent screen can be inserted up to $1.5 \mathrm{~m}$.

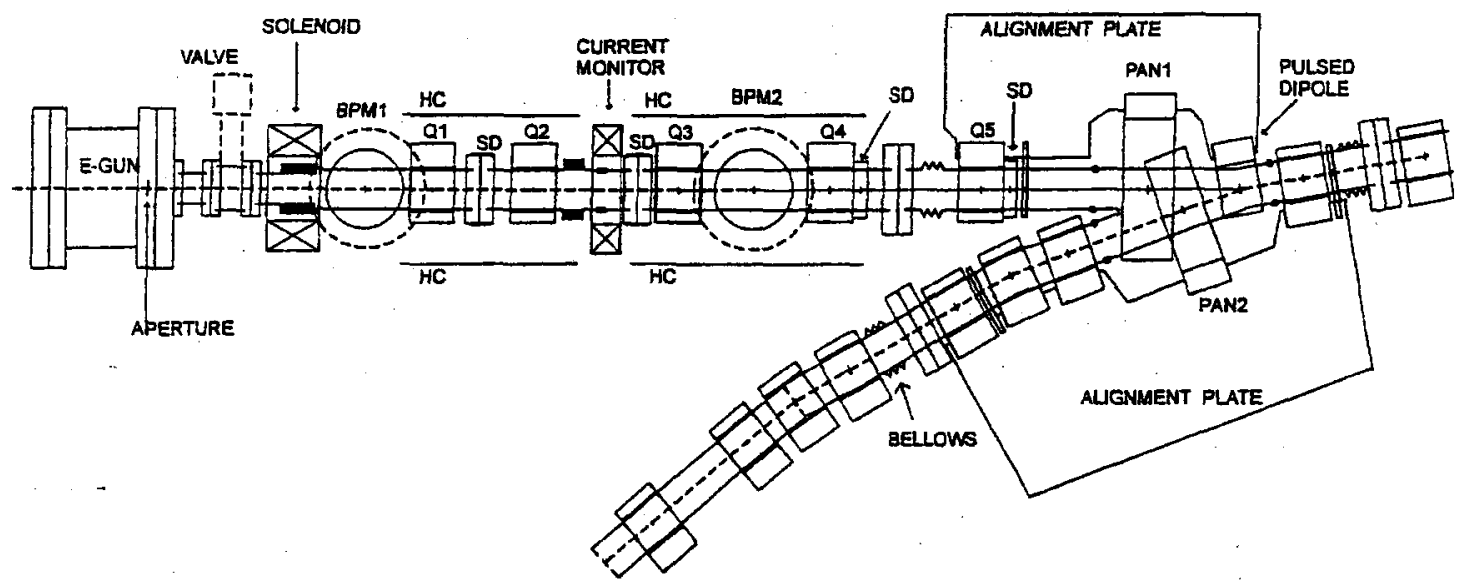

Fig. 6. Injector system layout. $Q=$ quadrupole; $P A N=$ Panofsky quad.; $\mathrm{SD}=$ short steering dipole; $\mathrm{HC}=$ Helmholtz conductors for earth field compensation; and BPM = beam position monitor. For scale, $Q 1$ to $Q 5$ is $72 \mathrm{~cm}$.

the beam dimensions. The design of the short steering dipoles (SD) is complete. A short glass section with bellows is included for a fast Pearson current transformer monitor.

\section{Panofsky quadrupoles}

The design concept for the cylindrical printedcircuit dipoles and quadrupoles has been described [5]. Since then, the algorithm for the azimuthal spacing of the active (axial) conductors has been improved. It is as follows: $\sin \left(m \theta_{\mathrm{n}}\right)=1-\left(2 z_{n} / k L\right)$ where $\theta$ is the azimuthal angle, $m=1$ or 2 for dipoles or quadrupoles, $z$ is the half-length of the $n$th axial conductor ( $z=0$ at the center) and $L$ is the length of the device. The inactive (transverse) conductors are uniformly spaced, e.g., $\Delta z=L / 42$ for the ring dipoles with 20 loops. The constant $k$ is near unity. A program was written based on the algorithm and, combined with a 3-D magnetics program and suitable axial integration, leads to a CAD file given to a printed-circuit fabricator.

Preliminary designs have been reported for the pulsed devices based on a compromise between field quality and the necessity for fast rise/fall time [6. A single layer circuit based on ten turns was adopted to reduce the inductance. The magnetics 


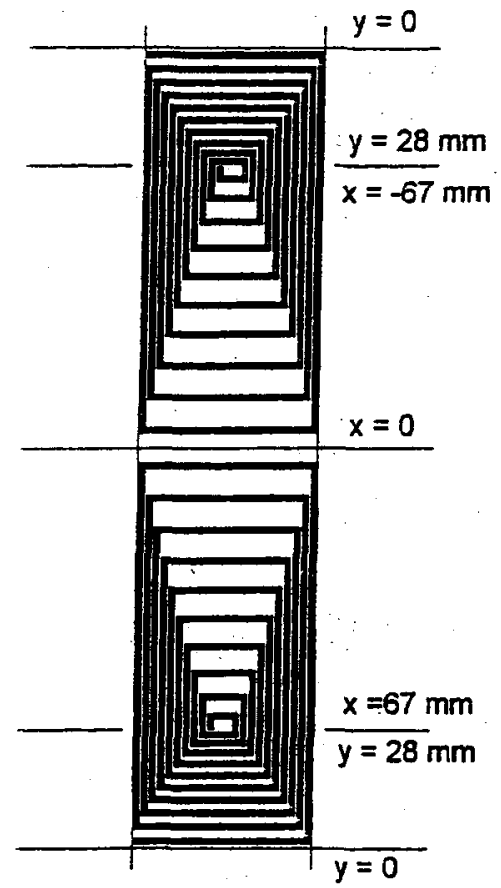

Fig. 7. Pattern calculated for the upper half of PAN2. See text for 3-D visualization.

design is complete and is identical for both PAN1 and PAN2. The latter is scaled down slightly to fit inside PAN1. The field errors were studied and parameters adjusted using the same basic procedure as outlined above for the cylindrical case.

Fig. 7 shows the circuit pattern of the most recent design, represented in 2-D, for the upper half of the Panofsky quadrupole. The 3-D pattern can be visualized by folding the outer conductors down at each of the corner $(y=28 \mathrm{~mm})$ lines. The calculated peak current is $28 \mathrm{~A}$ but the heating is negligible with a small duty factor. As in the cylindrical case, the field quality is optimized by calculating the integral gradient as a function of azimuth and radius and comparing with the gradient on and near the axis. After optimization, the deviations from ideal range from $0.02 \%$ at $5 \mathrm{~mm}$ radius to $0.6 \%$ at $17.5 \mathrm{~mm}$ radius. A scale model was built. When the final quadrupoles are built, the deviations will be checked using UMER's rotating coil apparatus [7].

\section{References}

[1] P.G. O'Shez, M. Reiser et al., University of Maryland Electron Ring (UMER), Nucl. Instr. and Meth. A 464 (2001) 646, these proceedings; M. Reiser et al., The Maryland Electron Ring for Investigating Space-ChargeDominated Beams in a Circular FODO System, Proc. 1999 Part. Accel. Conf., p. 234.

[2] T. Godlove et al., The $10 \mathrm{keV}$ Injector for the University of Maryland Electron Ring Project, Proc. 1999 Part. Accel. Conf., p. 1970.

[3] W.B. Hermannsfeldt, EGUN-Electron Optics and Gun Design, SLAC Rept: 331, October 1988.

[4] S. Bernal et al, Transport of a Space-Charge Dominated Electron Beam in a Short-Quadrupole Channel, Phys. Rev. Special Topics-Accel. \& Beams 1, 044202 (1998).

[5] T.F. Godlove, S. Bernal, M. Reiser, Printed-Circuit Quadrupole Design, Proc. 1995 Part. Accel. Conf., 2117 (1996).

[6] Y. Li et al., Design, Simulation and Test of Pulsed Panofsky Quadrupoles, Proc. 1999 Part. Accel. Conf., p. 3369.

[7] W.W. Zhang et al., Magnetic Field Measurements of Printed-Circuit Quadrupoles and Dipoles, Proc. 1999 Part. Accel. Conf., p. 3372. 


\title{
Experiments with Space Charge Dominated Beams for
}

\section{Heavy Ion Fusion Applications*}

P.G. O’Shea, R.A. Kishek, M. Reiser, B. Beaudoin, S. Bernal, Y. Cui, A. Diep,

D. Feldman, M. Glanzer, T.F. Godlove, I. Haber, J. Harris, H. Li, J. Neumann,

B. Quinn, M. Qurius, M. Snowel, A. Valfells, M. Virgo, M. Walter, R. Yun, Y. Zou

Institute for Research in Electronics and Applied Physics, Univ. of Maryland, College Park, MD 20742-3511, USA

\begin{abstract}
A detailed understanding of the physics of space-charge dominated beams is vital in the design of heavy ion inertial fusion (HIF) drivers. In that regard, low-energy, high-intensity electron beams provide an excellent model system. The University of Maryland Electron Ring (UMER), currently being installed, has been designed to study the physics of space-charge dominated beams with extreme intensity in a strong focusing lattice with dispersion. At $10-\mathrm{keV}$ and $100 \mathrm{~mA}$, the beam from the UMER injector has a generalized perveance as much as 0.0015 , corresponding to that of proposed HIF drivers. Though compact (11-m in circumference), UMER will be a very complex device by the time of its completion (expected 2003). We will present an update on the construction as well as recent experimental results.
\end{abstract}

Keywords: Space-charge-dominated beams, Circular Accelerators, Heavy ion inertial fusion 


\section{INTRODUCTION}

The energy requirements of Heavy Ion Inertial Fusion (HIF) imposes stringent requirements on the drivers used. A typical design for a driver involves the acceleration of multiple very-high intensity beams while maintaining the beam quality (Barnard et al. 2001). Low emittance is needed to enable focusing onto the target, and halo formation must be avoided in order to prevent activation of the accelerator structures (Friedman et al. 1999). At the University of Maryland, we continue to examine these issues using low-cost experiments with scaled electron beams (O'Shea et al. 2001), filling in an important gap in long-path experiments on intense beams.

Currently, there are a number of important experiments being conducted at the Virtual National Laboratory for Heavy Ion Fusion (VNL) which address particular aspects of building a Heavy Ion Fusion Driver. The Injector experiment at Lawrence Livermore National Laboratory (LLNL), described by Ahle et al. (2002), for example addresses the generation of the required high-current beams, while the High Current Experiment (HCX) at Lawrence Berkeley National Laboratory (LBNL), described by Seidl et al. (2002), examines the manipulation of such fullscale beams over short distances. Whereas a few years ago the scaled ion recirculator at LLNL (Ahle et al. 1999) promised a platform for conducting long-path experiments using scaled beams, the unfortunate termination of that project meant there will not be any long-path experiments conducted within VNL in the near future. The HIF effort has therefore relied considerably on its strong capabilities for simulation.

The University of Maryland Electron Ring (UMER) described by O'Shea et al. (2001) is designed as a low-energy recirculator using induction modules and with its parameters scaled to match Heavy Ion Fusion drivers. Scheduled to come on-line by the middle of 2003, UMER will 
provide the longest-path among existing high intensity machines for conducting experiments, and will also be useful in benchmarking computer codes used in machine design, such as WARP (Grote et al. 1996). In addition to UMER, we have a number of side experiments that use similarly scaled beams, particularly the Long Solenoid Experiment (LSE), designed by Cui et al. (2001) to study longitudinal beam physics in a straight geometry.

The unknown territory in the extreme space-charge dominated regime will be very challenging and should provide a wealth of new phenomena. UMER will permit experimental investigation of at least some of the many issues affecting beam quality in an HIF driver, for instance, longitudinal-transverse coupling, the behavior of bunch ends, and the longitudinal resistive wall instability, to name a few.

\section{UMER DESIGN FEATURES}

Table 1 lists some relevant parameters of UMER; more information can be found on the UMER WEBSITE. The beam is drifting at $10 \mathrm{keV}$, and 36 FODO cells around each turn provide transverse focusing, while 3 induction gaps provides longitudinal focusing. In a future stage, the induction gaps will also be used to accelerate the beam to $50 \mathrm{keV}$. At the present $\beta$ of 0.2 , the beam is nonrelativistic and has a similar velocity to HIF driver beams. A current of 100 $\mathrm{mA}$ ensures that the (generalized) perveance is also similar to that of HIF beams. The beam intensity can be quantified in a number of ways, for example using the dimensionless intensity parameter, $\chi$, defined in O'Shea et al. (2001) as the ratio of the space-charge force to the external focusing force at the beam radius. The tune depression is expressed in terms of $\chi$ as $\frac{\nu}{v_{0}}=\frac{k}{k_{0}}=\sqrt{1-\chi}$, and the plasma wavenumber $\mathrm{k}_{\mathrm{p}}$ as $\frac{k_{p}}{k_{0}}=\sqrt{2 \chi}$. HIF beams will likely operate with 
$0.89<\chi<0.98$. With a zero-current phase advance per period of $\sigma_{0}=76^{\circ}$, a nominal emittance of $\varepsilon_{\mathrm{n}}=10 \mu \mathrm{m}$ and beam radius a $=1 \mathrm{~cm}$, UMER can attain this region of extreme intensity.

\section{$<$ TABLE $1>$}

The electron bunch is injected into the ring at a repetition rate of $60 \mathrm{~Hz}$ or less from the injector system (Bernal et al. 2001) with the help of a DC Panofsky quadrupole and a pulsed dipole. The bunch can be extracted within the first turn or after any number of turns with a system that duplicates the features of the injector line except that the electron gun is replaced by a large diagnostic chamber with phosphor screen, emittance meter and energy analyzer (Cui et al. 2001; Valfells et al. 2001).

When complete, the ring magnetic lattice will consist of over 140 quadrupoles, dipoles and steering magnets. The typical focusing gradients and bending fields are on the order of 5 Gauss/cm and 10 Gauss, respectively. The use of iron-based magnets is impractical for such low fields. Therefore, the UMER magnets are based on an iron-free printed circuit (PC) design (Zhang et al. 2000) which, while fabricated to a very high tolerance, considerably lowers the cost of the project. A new feature that we have introduced recently is a quadrupole with electronically adjustable skewness (roll). The feature allows us to both correct for residual skewness in our system, and also to deliberately introduce skewness at various location in the ring for beam dynamics studies ( $\mathrm{Li}$ et al. 2001).

The design of the electron gun and simulations of its performance are described elsewhere (Kehne et al. 2001; Haber et al. 2001). A grid near the cathode can be used to generate perturbations as means of investigating instabilities. In addition, we have been investigating the possibility of producing ultra-short (1 ns) current pulses on top of the main pulse. We have 
achieved this by using a nitrogen laser as a photocathode drive laser on the dispenser cathode (Feldman et al. 2001). This will allow us to create perturbations on the beam that are well located in time and space. The evolution of such a perturbation will allow us to study dissipative processes, e.g. resistive wall phenomena.

To allow detailed comparison between theory and experiment, UMER will have a comprehensive set of beam diagnostics. Each of the 13 diagnostic stations around the ring will have a phosphor screen and capacitive beam position monitor (Harris et al. 2001). In addition, fast current monitors and resistive beam position monitors will also be installed. A sophisticated diagnostic end-chamber has been fabricated by FM Technologies. The chamber houses emittance meters of the slit-wire and pepper-pot types; a retarding-field energy analyzer with $\mathrm{eV}$ resolution for energy and energy spread measurements; a movable phosphor screen with 1.5 meter travel for insertion into the complete transport line; and a Faraday cup for current measurement.

\section{INITIAL EXPERIMENTAL RESULTS}

The initial emittance was characterized using the pepper pot method (Fig. 1), which provided an estimate of $15 \mu \mathrm{m}$ for the $4 \mathrm{x}$ rms normalized emittance. This is in general agreement with slitwire data we have recently obtained. Figure 1 indicates that the local temperature of the beam varies with radius.

\section{$<$ FIGURE 1 $>$}

To measure the energy spread of the beam, we have successively evolved the design of a parallel-plate electrostatic retarding-voltage energy analyzer (Cui et al. 2001; Valfells et al. 2001). The first improvement consisted of adding a collimating cylinder attached to the retarding mesh. The cylinder collimates the particle trajectories such that they are normally incident on 
the mesh, hence reducing any errors from a transverse angle or spread in velocities. Simulations show the resolution of the device is about $8 \mathrm{eV}$ (at $10 \mathrm{keV}$ ), and the measured values of the energy spread are just slightly greater than this value (Fig. 3). This is a factor of 5 improvement over the previous design.

While the measured energy spread meets our specification of $20 \mathrm{eV}$, we are continuing to improve our diagnostic. By electrically separating the collimating cylinder from the retarding mesh (Cui et al. 2001), we gain additional control over the focusing of the trajectories, and simulations predict an extra factor of 10 in the resolution of the device, i.e., down to $10^{-4}$ ! This $3^{\text {rd }}$-generation energy analyzer is currently being tested.

$<$ FIGURE 2>

$<$ FIGURE 3>

\section{PLANS}

At present (May 2002), we are engaged in a phased installation of the ring. The first two out of 18 ring sections are currently being installed, and we are therefore about to complete 40 degrees shortly. The installation is done gradually to permit us to take measurements using the end diagnostic chamber around the entire perimeter of the ring during the first turn. This phased installation is scheduled to be completed by mid- 2003 .

Upon completion of installation, we will begin multi-turn operation, targeting 100 turns at low current $(10 \mathrm{~mA})$ and 10 turns at high current $(100 \mathrm{~mA})$, while maintaining beam emittance to within a factor of 4. At the end of that phase, we intend to upgrade UMER to a fast cycling synchrotron that accelerates the beam to $50 \mathrm{keV}$ over 50-100 turns to study resonance crossing. 


\section{ACKNOWLEDGEMENTS}

We wish to acknowledge Richard York and his colleagues at Michigan State University as well as FM Technologies, Inc. for their assistance with the design and construction of UMER components. This project is fully funded by the US Dept. of Energy grant numbers DEFG0294ER40855 and DEFG02-92ER54178. 


\section{REFERENCES}

Note: the following symbols are used to abbreviate certain proceedings.

"PAC01" means "Proceedings of the 2001 Particle Accelerator Conference, Chicago, IL, edited by LUCAS P. \& WEBER, S., IEEE Cat. No. 01CH37268".

"PAC99" means "Proc. IEEE 1999 PAC Conference, New York City, NY, edited by LUCCIO, A. \& MACKAY, W. IEEE Cat. No. 99CH36366”.

AHLE, L. (2002). These Proceedings.

AHLE, L., SANGSTER, T.C., AUTREY, D., BARNARD, J., CRAIG, G., FRIEDMAN, A., GROTE, D.P., HALAXA, E., LOGAN, B.G., LUND, S.M., MANT, G., MOLVIK, A.W., SHARP, W.M., EYLON, S., DEBELING, A. \& FRITZ, W. (1999). PAC99, pp. 3248-3251.

BARNARD, J.J., AHLE, L.E., BANGERTER, R.O., BIENIOSEK, F.M., CELATA, C.M., FALTENS, A., FRIEDMAN, A., GROTE, D.P., HABER, I., HENESTROZA, E., DE HOON, M.J.L., KARPENKO, V.P., KISHEK, R.A., KWAN, J.W., LEE, E.P., LOGAN, B.G., LUND, S.M., MEIER, W.R., MOLVIK, A.W., SANGSTER, T.C., SEIDL, P.A. \& SHARP, W.M. (2001). Planning for an Integrated Research Experiment. Nuclear Instruments and Methods, A464, 621-628.

BERNAL, S., LI, H., VIRGO, M., KWON, S.P., HOLLAND, M., KISHEK, R.A., VALFELLS, A., GODLOVE, T., O'SHEA, P.G., REISER, M., YUN, V. \& KEHNE, D. (2001). Beam Test of the $10 \mathrm{KeV}$ Injector for the University of Maryland Electron Ring (UMER). PACO1, pp. $2129-2132$. 
CUI, Y., BERNAL, S., KISHEK, R.A., O’SHEA, P.G., RAHIMI, N., REISER, M., VALFELLS, A. \& YUN, V. (2001). Design Studies for an Experiment to Measure Energy Spread Evolution Through a Solenoidal Focusing System. PAC01, pp. 2976-2979.

FELDMAN, D.W., VALFELLS, A., NEUMANN, J., HARRIS, J., BEAUDOIN, B. \& O'SHEA, P.G. (2001). Combined Thermionic and Photoelectric Emission From Dispenser Cathodes. PAC01, pp. 2132-2135.

FRIEDMAN, A., BARNARD, J.J., CELATA, C.M., CRAIG, G.D., DE HOON, M.J.L., FALTENS, A., GROTE, D.P., LEE, E.P., SHARP, W.M., SONNENDRUCKER, E., HABER, I. \& KISHEK, R.A. (1999). Beam Dynamics Studies for Heavy Ion Fusion Drivers. PAC99, pp. 1830-1833.

GROTE, D.P., et. al., (1996). Fus. Eng. \& Des. 32-33, 193-200.

HABER, I., BERNAL, S., KISHEK, R.A., O'SHEA, P.G., REISER, M., VALFELLS, A. \& GROTE, D.P. (2001). Computer Simulation of the UMER Electron Gun. PAC01, pp. 29522955.

HARRIS, J., BERNAL, S., O'SHEA, P.G., PRUESSNER, M., QUINN, B., REISER, M. \& YUN, V. (2001). A Fast Beam Position Monitor for UMER. PAC01, pp. 1387-1390.

KEHNE, D., GODLOVE, T., HALDEMANN, P., BERNAL, S., GUHARAY, S., KISHEK, R., Y. LI, O'SHEA, P., REISER, M., YUN, V., ZOU, Y. \& HABER, I. (2001). Nuclear Instruments and Methods, A464, 605-609.

LI, H., BERNAL, S., KISHEK, R.A., GODLOVE, T., O'SHEA, P.G. \& REISER, M. (2001). Printed-Circuit Magnets for the University of Maryland Election Ring (UMER)-New Developments. PAC01, p. 1802-1805. 
O'SHEA, P.G., REISER, M., KISHEK, R.A., BERNAL, S., LI, H., PRUESSNER, M., YUN, V., CUI, Y., ZHANG, W., ZOU, Y., GODLOVE, T., KEHNE, D., HALDEMANN, P., \& HABER, I. (2001). The University of Maryland Electron Ring (UMER). Nuclear Instruments and Methods, A464, 646-652.

SEIDL, P. (2002). These Proceedings.

UMER WEBSITE: http://www.ireap.umd.edu/umer

VALFELLS, A., BERNAL, S., CUI, Y.P., KISHEK, R.A., O'SHEA, P.G., QUINN, B., REISER, M., VIRGO, M., YUN, V. \& NISHIURA, M. (2001). Energy Analyzer Experiments for the University of Maryland Electron Ring. PAC01, pp. 3582-3585.

ZHANG, W.W., BERNAL, S., LI, H., GODLOVE, T., KISHEK, R.A., O’SHEA, P.G., REISER, M., YUN, V. \& VENTURINI, M., (2000). PRST-AB 3, 122401. 


\section{TABLES}

TABLE 1. UMER design specifications

\begin{tabular}{lc}
\hline Energy & $10 \mathrm{keV}$ \\
$\beta(=\mathrm{v} / \mathrm{c})$ & 0.2 \\
Current & $\leq 100 \mathrm{~mA}$ \\
Generalized perveance & 0.0015 \\
Emittance, 4x rms, norm & $10 \mu \mathrm{m}$ \\
Pulse Length & $40-100 \mathrm{~ns}$ \\
Circumference & $11.52 \mathrm{~m}$ \\
Lap time & $197 \mathrm{~ns}$ \\
Pulse repetition rate & $60 \mathrm{~Hz}$ \\
Mean beam radius & $\leq 1 \mathrm{~cm}$ \\
FODO period & $0.32 \mathrm{~m}$ \\
0-current phase advance, $\sigma_{0}$ & $76^{\circ}$ \\
O-current Betatron tune, $v_{0}$ & 7.6 \\
Tune Depression & $\geq 0.2$ \\
\hline
\end{tabular}




\section{FIGURE CAPTIONS}

FIGURE 1. Pepperpot image of the beam from the UMER gun.

FIGURE 2. Current pulse on energy analyzer with zero bias voltage.

FIGURE 3. Energy spread measured at the head and tail with the current pulse in Fig. 2. 


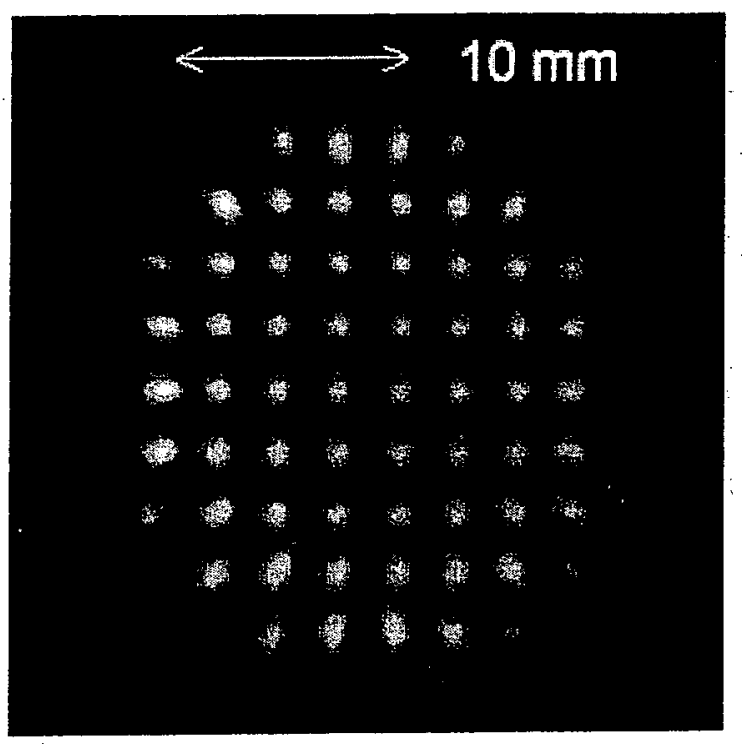




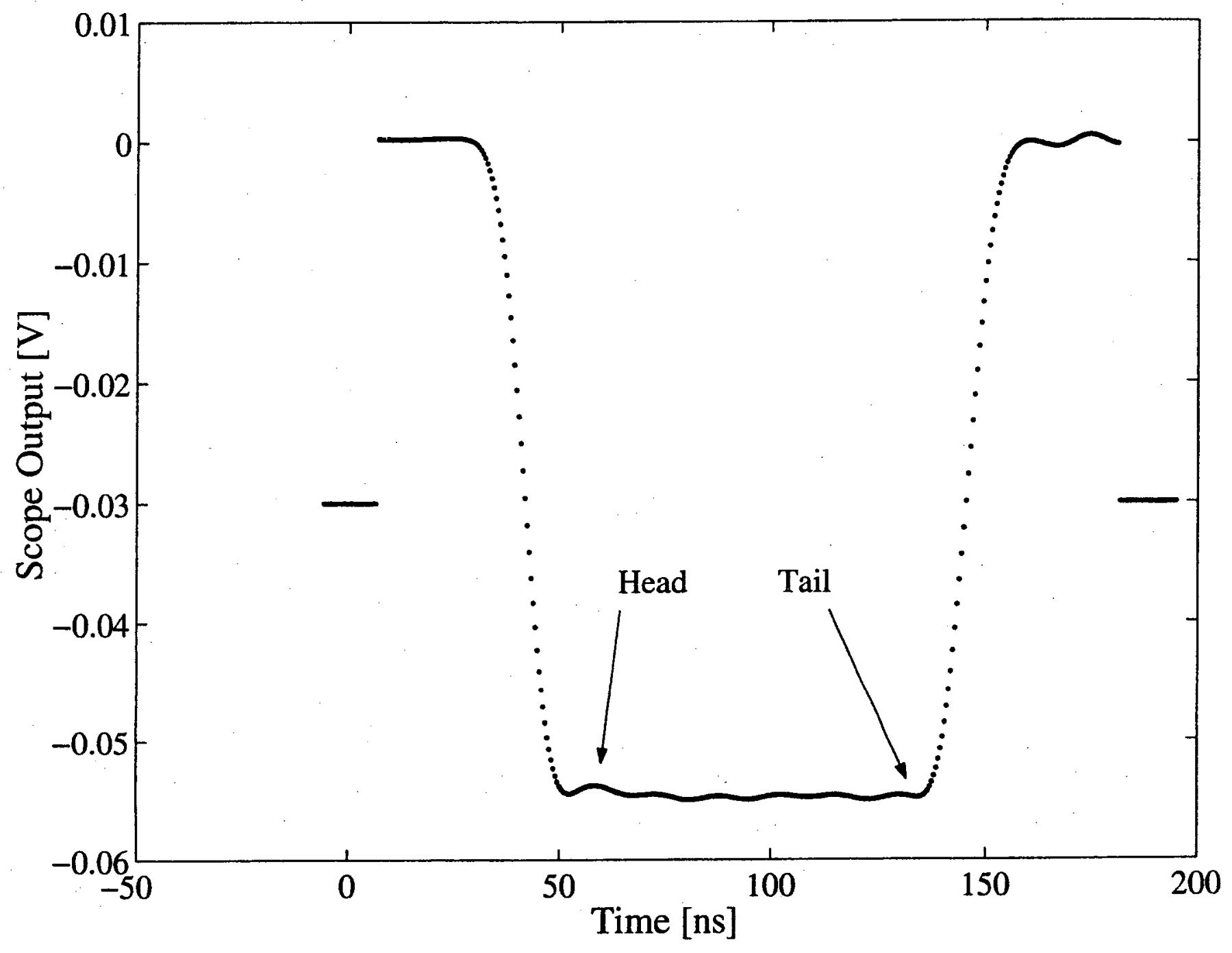




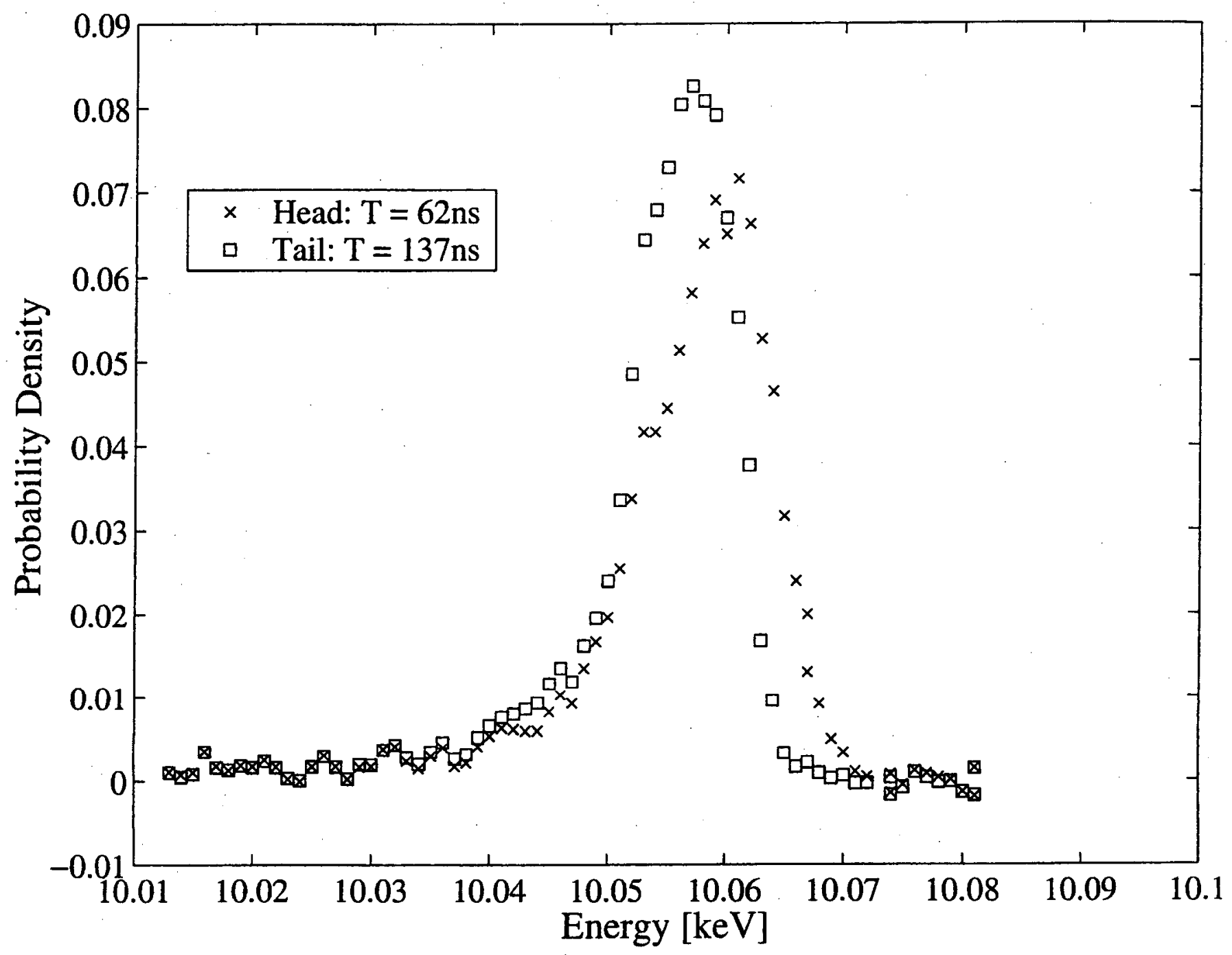




\title{
Simulations and experiments with space-charge-dominated beams*
}

\author{
R.A. Kishek ${ }^{1}$, S. Bernal, C.L. Bohn ${ }^{\#}$, D. Grote ${ }^{\dagger}$, I. Haber, H. Li, P.G. O’Shea", \\ M. Reiser, M. Walter \\ Institute for Research in Electronics and Applied Physics, Univ. of Maryland, College Park, MD 20742-3511, USA \\ * Also Dept. of Electrical and Computer Engineering, Univ. of Maryland, College Park, MD \\ † Lawrence Berkeley National Laboratory, Berkeley, CA \\ \# Northern Illinois University, DeKalb, IL; also Fermi National Lab, Batavia, IL
}

\begin{abstract}
Beams in which space charge forces are stronger than the force from thermal pressure are nonneutral plasmas, since particles interact mostly via the long-range collective potential. An ever-increasing number of applications demand such high-brightness beams. The University of Maryland Electron Ring (UMER) [P.G. O'Shea, et al., NIM $A$ 464, 646 (2001)], currently under construction, is designed for studying the physics of space-charge-dominated beams. Indirect ways of measuring beam emittance near the UMER source produced conflicting results, which were resolved only when a direct measurement of phase space indicated a hollow velocity distribution. Comparison to self-consistent simulation using the particle-in-cell code WARP [D.P. Grote, et al., Fus. Eng. \& Des. 32-33, 193-200 (1996)] revealed sensitivity to the initial velocity distribution. Since the beam is born with nonuniformities and granularity, dissipation mechanisms and rates are of interest. Simulations found that phase mixing by means of chaotic particle orbits is possible in certain situations, and proceeds much faster than Landau damping. The implications for using beams to model other N-body systems are discussed.
\end{abstract}

'e-mail: ramiak@ebte.umd.edu 


\section{INTRODUCTION}

A charged particle beam in which the repulsive space charge forces are stronger than the outward force from thermal pressure is called space-charge dominated [1]. In this regime, the Debye length is smaller than the beam size, and so particles interact mostly via the long-range collective potential, as in a (nonneutral) plasma. Whereas many emerging accelerators strive for higher beam intensities, most beams are actually created in this intensity regime near their source, but eventually the space charge forces relatively weaken due to acceleration, transverse compression, and an increase in thermal pressure caused by emittance growth. Even when space charge is not an important factor in the behavior of the bulk of an accelerator system, it can therefore still be important to include the influence of space charge in system design. To begin with, the characteristics of the source region determine the initial state of the beam, and characteristics of the initial state such as beam emittance and halo formation [2] are often quite important to downstream behavior. Less apparent is that space-charge collective modes that persist for hundreds of focusing periods can be excited in the source region under some conditions [3]. It is therefore important to understand the effects of intense space charge on beam dynamics, since the initial beam state can affect downstream emittance growth, halo formation, and the broadening of ring resonances. The University of Maryland Electron Ring (UMER) [4], currently under construction, is designed for conducting beam physics experiments at extreme intensities in a circular lattice, and will serve as low-cost model of high-intensity accelerators for a variety of applications, such as heavy-ion fusion drivers, the low-energy end of high intensity electron linacs, ion booster synchrotrons, muon colliders, and spallation neutron sources. 
Since a space-charge-dominated beam can be viewed as a one-component non-neutral plasma where the averaged focusing forces take the place of a fixed neutralizing background, it is a medium that can carry waves or collective oscillations [5]. Yet despite the fact that thermal pressure is much less than space charge forces, the beam kinetic temperature cannot be completely neglected. As we shortly present, the detailed evolution of space charge waves depends not only on the rms temperature or emittance, but also on the detailed velocity space distribution. After a brief overview of UMER in the next section, we present experimental measurements of the beam distribution function near the UMER electron gun. Various indirect methods of measurement give us conflicting results, which cannot be reconciled except by assuming a hollow velocity distribution, a fact verified by direct mapping of phase space using a pepper-pot. We augment the experimental results with self-consistent particle-in-cell simulations using the particle-in-cell code WARP, developed at LLNL [6]. The beam emerging from the source is far from equilibrium, instead carrying waves and granular microstructures. We are therefore interested in dissipation mechanisms and rates and, in particular, the possibility of rapid phase mixing by means of chaotic particle orbits. An ongoing simulation study is highlighted in Section IV, and its implications are discussed.

\section{OVERVIEW OF UMER}

Table 1 lists some relevant parameters for UMER, which is discussed in more detail in Ref. 3, and on the website http://www.ireap.umd.edu/umer. The beam presently drifts at $10 \mathrm{keV}$, so the $\beta$ of 0.2 implies the beam is nonrelativistic. Three induction gaps distributed equidistantly around the ring provide longitudinal focusing, and will also be used to accelerate the beam to 50 $\mathrm{keV}$ in a future stage. Transverse focusing is provided by 36 alternating-gradient quadrupole (FODO) cells around the 11.52 meter circumference of the ring. Each pair of FODO cells, along 
with two $10^{\circ}$ bends, is installed on one out of 18 mechanical sections. As of Nov. 2002, the injector and the first four ring sections have been installed and characterized, providing data from 9 bends. Additional sections are assembled and ready to be installed as part of a phased installation program. The entire ring is scheduled to be complete during the summer of 2003.

The beam intensity can be quantified in a number of ways, for example using the dimensionless intensity parameter, $\chi$ (defined in ref. [4] as the ratio of the space-charge force to the external focusing force at the beam radius). The space charge depresses the betatron oscillation frequency by a ratio $\frac{\omega_{\beta}}{\omega_{\beta o}}=\frac{k}{k_{o}}=\sqrt{1-\chi}$, while the plasma frequency $\omega_{p}$ is enhanced: $\frac{\omega_{p}}{\omega_{\beta_{0}}}=\sqrt{2 \chi}$. Another parameter of importance, which is related directly to the value of $\chi$, is the ratio of beam radius, $r_{b}$, to the Debye length $\lambda_{D}=v_{t h} / \omega_{p}$, where the beam kinetic energy is $1 / 2 \mathrm{mv}_{\text {th }}{ }^{2}$ and the plasma frequency $\omega_{\mathrm{p}}=\left(4 \pi \mathrm{ne}^{2} / \mathrm{m}\right), \mathrm{n}$ the particle density, e the particle charge and $\mathrm{m}$ the particle mass. For intensities above $\chi=0.5$, the Debye length becomes significantly smaller than the beam radius, and the beam is able to support collective space-charge oscillations, since those oscillations usually do not occur at wavelengths less than $\lambda_{D}$.

With the design beam current of $100 \mathrm{~mA}$, an initial emittance of $\varepsilon_{\mathrm{n}}=12-15 \mu \mathrm{m}$ and a zero-current phase advance per period of $\sigma_{0}=76^{\circ}$ (giving a beam radius a $=1 \mathrm{~cm}$ ), UMER can achieve an intensity of $\chi=0.97$, which is near the extreme intensity. The intensity of the UMER beam can be reduced over a wide range (down to $\chi \sim 0.35$ ) by changing the anode-cathode spacing, the beam energy, the potential at the cathode grid, or by changing the aperture size in the beam collimator at the exit of the gun. In this paper, emittance refers to normalized emittance calculated according to the formula: 


$$
\varepsilon_{x n}^{2}=16 \beta \gamma\left(\left\langle x^{2}\right\rangle\left\langle x^{\prime 2}\right\rangle-\left\langle x x^{\prime}\right\rangle^{2}\right)
$$

where the $\langle\ldots\rangle$ indicate taking the moments over the 4-D transverse beam distribution $\mathrm{f}\left(\mathrm{x}, \mathrm{x}^{\prime}, \mathrm{y}, \mathrm{y}^{\prime}\right)$. Here $\mathrm{x}$ is the particle position in the horizontal direction (minus the beam centroid), $\mathrm{x}^{\prime}$ is the particle angle with the reference trajectory, $\mathrm{v}_{\mathrm{x}} / \mathrm{v}_{\mathbf{z}}$ (minus that of the beam centroid), $\beta$ is the beam velocity normalized to the speed of light, and $\gamma$ is the relativistic factor. A similar expression holds for the $\mathrm{y}$ emittance, $\varepsilon_{\mathrm{yn}}$.

To allow detailed comparison between theory and experiment, UMER has a comprehensive set of beam diagnostics. In addition to the 13 diagnostic stations around the ring with phosphor screens and capacitive beam position monitors (BPMs), a number of resistive BPMs and fast current monitors will also be installed. A sophisticated diagnostic end-chamber is currently in operation and will eventually reside at the end of the extraction section. The chamber houses emittance meters of both the slit-wire and pepper-pot types; a retarding-field energy analyzer with $\mathrm{eV}$ or sub-eV resolution for energy and energy spread measurements [7]; a movable phosphor screen with up to 1.5 meter travel for insertion into the extraction transport line; and a Faraday cup for current measurement. $\mathrm{CCD}$ cameras record the time-integrated images of the beam from the phosphor screens.

\section{SIMULATING SOURCE AND INJECTOR EXPERIMENTS}

\section{A. Simulation code and model}

The simulations presented here employ the particle-in-cell code WARP [6] to selfconsistently simulate space charge effects in our experiments. The WARP code simulates space charge effects in 2D or 3D by advancing a large number of macroparticles in response to both 
the external applied fields and the self-fields. The self-fields are calculated on a mesh of sufficient resolution to capture the variations of the beam potential, and the particles are advanced using a leapfrog algorithm. WARP has been under development for nearly a decade, so it possesses a variety of models for various accelerator elements - magnets, induction gaps, apertures, etc. - as well as many different choices of field solvers, boundary conditions, and numerical algorithms. Many input beam distributions are also possible, of which the most commonly used for space-charge-dominated beams is the semi-Gaussian, in which the particle density is uniform across the beam, while the velocity distribution is Gaussian with uniform temperature.

Although most of the simulations reported here were modeled with the 2-1/2 D "slice" version of WARP (WARPxy), which solves for the transverse behavior while advancing a beam slice along s, some problems are inherently 3-dimensional. The source simulations employed the $\mathrm{r}-\mathrm{z}$ axisymmetric module, which is also used for modeling propagation and confinement of the beam ends and the retarding voltage energy analyzer.

\section{B. The UMER electron gun}

The electron gun is of Pierce geometry with a $4 \mathrm{~mm}$ radius cathode [8]. Changing the $\mathrm{A} / \mathrm{K}$ separation or the gird-cathode potential can vary the perveance. Since the accelerating potential across the gap is applied using a $\mathrm{DC} 10 \mathrm{kV}$ power supply, a grid located $0.15 \mathrm{~mm}$ from the cathode surface is biased negatively (usually at $40-50 \mathrm{~V}$ ) in order to impede the current flow until a positive pulse is applied to the grid. Since the separation between the wires in the rectangular grid pattern is $0.15 \mathrm{~mm}$, i.e. the same as the distance to the cathode, the field pattern in the vicinity of the emitter surface is complex, especially when beam space charge is included. Another grid (with $87 \%$ transmission) intercepts the beam in the anode plane in order to create 
an equipotential surface, as E-GUN simulations had shown this method reduces gun aberrations. The grids appear in a photograph taken without the beam (Fig. 1a). In addition, the UMER gun structure has a set of masks on a plate that can be rotated without breaking vacuum. This mask plate contains a pepper-pot mask for measuring transverse phase space, a five-beamlet quincunx pattern (similar to the five on the face of a die), as well as a series of round holes with various radii used to change the beam current and emittance to provide beams with widely different intensities.

\section{Measurement of the initial beam distribution}

\section{Shadow of anode grid}

One of the early mysteries in characterizing the UMER gun was estimating the emerging beam's emittance. A calculation of intrinsic emittance from the cathode radius and temperature results provides a value of $3.6 \mu \mathrm{m}$. This number however proved inconsistent with some of the measurements. For example, the beam size from transport experiments in the injector hinted at a much larger emittance $(\sim 12-15 \mu \mathrm{m})$. The interesting issue is that a P-screen positioned $6 \mathrm{~cm}$ downstream from the anode clearly shows a shadow of the anode grid pattern [9], as in Fig. 1 b. WARPxy simulations using the slice model and an initial semi-Gaussian distribution starting from the anode with the higher emittance have been unable to reproduce the grid pattern (Fig. 1c), as the shadow tends to wash out very quickly. Yet when the simulation emittance is reduced to near the intrinsic emittance, the grid shadow pattern can be seen in the simulation (Fig. 1d).

\section{Five-beamlet merging}

The emittance mystery compounded with additional data. Figure 2 shows phosphor screen images measured $104 \mathrm{~cm}$ downstream from the anode plane when the five-beamlet mask 
is used. This photograph is typical of the whole series of pictures taken along the injector using the moveable P-screen. Also shown on the same figure are simulations performed using the WARP PIC code in single slice mode [10]. The emittances labeled on the simulation curves shown in Fig. 2 are calculated by multiplying the thermal velocity assumed for the beamlets by the total area the beam would have at the anode plane in the absence of the mask. It is clear that the shape of the pattern downstream depends on the beam emittance. From a comparison between the simulated and measured pattern one infers that the best match occurs when the initial normalized emittance of the total beam is between $16 \mu \mathrm{m}$ to $18 \mu \mathrm{m}$. This value is a factor of 4 or 5 larger than intrinsic emittance and the near-laminar flow that would allow the shadow of the grid to persist for $6 \mathrm{~cm}$ !

\section{Pepper-pot measurements and hollow velocity distribution}

No satisfactory explanation for this behavior is possible with the simple semi-Gaussian model. Fortunately, we were able to directly measure the beam phase space using a pepper-pot mask built into the aperture wheel near the anode. The results, of which one photograph is shown in Fig. 3a, provide the major clue for resolving this mystery. The beamlets emerging from the $0.1 \mathrm{~mm}$ holes in the pepperpot expand to produce hollowed projections on the screen. These donuts indicate a hollowing of the velocity space of the beam, possibly caused by the distortion of the potential near the cathode by the (cathode) grid. Reproducing the pattern in the simulations is not simple, due to the 3-D nature of the problem and the disparity of scales between the cathode grid dimensions and the $\mathrm{A} / \mathrm{K}$ gap, making simulation difficult [11]. Nevertheless we were able to obtain an approximation of this effect by injecting a beam with an artificially hollowed velocity distribution into the slice code simulations at the anode. Various implementations of hollowness have been attempted, for instance one with rectangular symmetry 
to mimic the hollowness that could be created by the cathode grid. The results shown in the remainder of this paper use a radially hollowed velocity (i.e., possessing azimuthal symmetry) that is obtained by rotating a Gaussian distribution with width $\mathrm{v}_{\mathrm{th}}$ and offset $\alpha$ from the origin around a circle of radius $\alpha$. The cross-section of such a velocity distribution on the $v_{x}$ axis is shown in Fig. 4, along with a snapshot of $y$-y' trace space. Henceforth we refer to this distribution as "annular-Gaussian", noting that the density distribution is uniform as in the semiGaussian.

The parameters $\alpha$ and vth are obtained empirically by fitting to experimental data. For this paper, we use a width for the Gaussian corresponding to the intrinsic emittance, and a radius of rotation $\alpha$ selected to result in an rms beam emittance of $14 \mu \mathrm{m}$. These parameters can be varied of course, considering the pepper-pot images in the experiment itself change with the bias voltage of the cathode grid. A simulation of the pepper-pot with this distribution (Fig. 3b) results in a picture qualitatively similar to the experiment. Furthermore, this distribution reproduces both the shadow of the grid (Fig. 3c) and the five-beamlet experiment, as well as the injector experiments.

Similarly by using $\mathrm{r}-\mathrm{z}$ simulations of the gun structure starting from, but not including the cathode grid [11], we were able to reproduce the experimentally measured features only when hollowness in the velocity space of the injected distribution was assumed. The agreement using the $r-z$ code was significantly better in some respects, but the point here is that even a crude 2-D model with a hollowed velocity can qualitatively explain experimental features that cannot be explained with a semi-Gaussian. 


\section{Propagation in the injector}

Fig. 5a shows a series of beam photographs taken along the injector using a moveable Pscreen [12]. The photographs demonstrate evolution of the beam as it propagates through the matching system, and exhibits space charge waves similar to one discovered in previous experiments [13]. In addition, the experimental photographs exhibit some fine-structured patterns that were not observed in the previous experiments. Since the primary difference from the earlier experiments is the added presence of the grids in the gun, we suspect that to be the main factor resulting in this fine structure. Simulations with WARPxy [Fig. 5b,c], using initially uniform density distributions, but two different velocity distributions having the same rms values, demonstrate a resulting difference in the detailed density distribution downstream. In particular, the annular-Gaussian distribution used earlier more closely resembles the experimental results, although the agreement is not exact.

The emerging conclusion is that beam dynamics in an accelerator can be very sensitive to the details of the 6-D distribution at the source. Even if a beam appears to be well-behaved in configuration space, distortions in velocity space can reappear as density structures later downstream. Note that this fine structure persists for a long time. In our case the hollowness in the velocity distribution caused by the cathode grid persists as fine structure in the beam even in the latest experimental photographs 9 bends past the injector, i.e. several plasma periods from the cathode. While the rms beam size and other $2^{\text {nd }}$ order moments are affected only by the rms emittance as calculated in Eq. (1), it is clear that the detailed density patterns including the propagation and phases of space charge waves are affected by the higher-order moments of the initial distribution. As we have shown, it is possible to construct two completely different distributions with identical emittances, such as a thermal distribution and one with two laminar 
"streams" propagating at an angle to each other, but obviously the beam dynamics will evolve differently for the different distributions when the details are considered.

\section{STUDIES OF MIXING BY MEANS OF CHAOTIC ORBITS}

A related issue, of fundamental importance to beam physics as well as plasma physics in general, is mixing and dissipation of charge density structures and granularities, such as those we measured in the UMER beam near the source. Beams with space charge are, like many plasmas, typically collisionless Hamiltonian systems where the detailed density distribution selfconsistently governs the dynamics via Poisson's equation. The questions of equilibration, damping, and reversibility are of fundamental importance in determining beam properties. For example, equipartitioning of anisotropic beams involves nonlinear energy transfer and evolution towards an isotropic meta-equilibrium [14]. It is important for the accelerator designer to know whether the exchange of energy in this process is reversible. Another example, while it may be possible to correct for emittance growth in a 3-D context by emittance compensation techniques [15], this compensation needs to be applied before any significant mixing has rendered the growth irreversible. The same question arises when considering any process manipulating a space charge-dominated beam; whether it is flat beam generation, bunch distortion due to CSR, matching through transitions in a linac, or emittance growth due to misalignments or dispersion. Furthermore, these issues are of importance beyond the plasma or beams community, as they apply to any n-body system interacting via long-range forces without collisions, as is the case in large stellar systems [16], for example.

In order to answer these questions we are conducting a large-scale simulation study of beam dynamics with a focus on individual particle orbits [17]. Whereas traditional PIC 
simulation is concerned mostly with the macroscopic behavior resulting from the interaction of macroparticles, we require sufficient numerical resolution so as to observe reliable macroparticle orbits and analyze them statistically. Our main concern is whether or not particles follow globally chaotic orbits, in which case they can phase mix quite rapidly at rates that can be calculated from theory [18].

To determine this, we seed large numbers of test particles concentrated in various locations in 4-D phase space. The remainder of the beam should have a sufficient number of particles and sufficiently-fine simulation mesh to produce a smooth and well-resolved potential at every step. Time steps are chosen small enough relative to the natural frequencies of the system to result in a sufficiently smooth trajectory. Particle advance is done using a symplectic leap-frog algorithm, which guarantees that the numerical simulation itself is reversible. Tracking the test particles in the self-consistent potential of the beam, we then look for exponential separation of orbits indicative of chaos, in which case we can calculate the finite-time Lyapunov exponents [19] for each initially-localized collection of test particles. Note that since in general we do not have equilibrium, the problem is time dependent. An example is shown in Fig. 6 comparing the "emittance" moments for five clumps of test particles (defined as in Eq. 1, but including only particles belonging to a particular clump) for each of two test cases: a round isotropic space-charge-dominated beam in a uniform focusing channel, and an otherwise similar beam having different initial emittances (hence temperatures) in $\mathrm{x}$ and $\mathrm{y}$. The anisotropic case has been observed to macroscopically relax to an isotropic beam on a very fast timescale (2-3 plasma periods) [14]. The mixing rate, defined as the growth rate of the "emittance" moment of each clump (indicative of the separation of nearby trajectories), proceeds much faster for the anisotropic beam, as evident from Fig. 6. Furthermore, this growth for the anisotropic beam fits 
closely to a straight line on a semi-log plot, indicating exponential growth. A comparison of trajectories in $x-y$ space of 20 sample test particle from one clump in each case indicates a qualitative difference in behavior [Fig. 7].

The fundamental significance of this type of study is that chaotic mixing can be found in many types of physical systems. Whereas large stellar systems are largely inaccessible outside of computer simulations, beam experiments can nevertheless be constructed to test hypotheses pertaining to fundamental questions in galactic dynamics.

\section{v. CONCLUSION}

Measurements of the space-charge-dominated beam in the UMER source and injector have been compared to self-consistent particle-in-cell simulations using the WARP code. The various indirect measurements exhibit mutual contradiction in the inferred value of emittance if a semi-Gaussian distribution is assumed. This contradiction has been removed once a direct measurement of phase space using a pepper-pot identified a hollowed velocity distribution. Even an ad-hoc use of a hollowed velocity distribution in the simulations resulted in much-improved agreement with experimental results. This emphasizes the indispensability of direct phase space measurements, as well as demonstrates the sensitivity of detailed beam distributions downstream to the velocity distribution at the source.

Dissipation of granularities and microstructures such as what has been observed in the experiment has also been studied using the WARP simulations. Results indicate rapid mixing by means of chaotic particle orbits can take place in certain circumstances. This raises the possibility of setting up charged particle beam experiments to model other and less-accessible Nbody systems such as galaxies. 


\section{ACKNOWLEDGEMENTS}

We wish to acknowledge David Kehne and FM Technologies, Inc., for the design and construction of the gun and diagnostic chamber. We are also grateful for many productive discussions with David Kehne, Alex Friedman, Henry Kandrup, Tom Antonsen, Steve Lund, Jean-Luc Vay, Ioannis Sideris, as well as other members of the UMER team. This work is performed under the auspices of the US Dept. of Energy under contracts no. DEFG0294ER40855, DEFG02-92ER54178, W-7405-Eng-48 and DE-AC03-76SF00098, as well as a Department of Education, NICADD grant. 


\section{REFERENCES}

[1] Martin Reiser, Theory and Design of Charged Particle Beams, (New York: John Wiley \& Sons, Inc., 1994).

[2] T.P. Wangler, Phys. Rev. ST-AB 1, 084201 (2000).

[3] S. M. Lund and R. C. Davidson, Phys. Plasmas, 5 (8), 3028 (1998).

[4] P.G. O'Shea, M. Reiser, R.A. Kishek, et al., NIM A 464, 646 (2001); Laser and Particle Beams, accepted for publication (2002).

[5] Ronald C. Davidson and Hong Qin, Physics of Intense Charged Particle Beams in High Energy Accelerators, (Singapore: World Scientific, 2001).

[6] D.P. Grote, A. Friedman, S.M. Lund, and I. Haber, Fus. Eng. \& Des. 32-33, 193-200 (1996).

[7] Y. Zou, Y. Cui, V. Yun, A. Valfells, R.A. Kishek, S. Bernal, I. Haber, M. Reiser, P.G. O'Shea, and J.G. Wang, PRST-AB 5, 072801 (2002); Y. Cui et al., Proc. 2001 Particle Accelerator Conf., Chicago, IL, ed. P. Lucas and S. Weber, I4 Cat. No. 01CH37268, p. 2976 (2001).

[8] D. Kehne, T. Godlove, P. Haldemann, et al., NIM, A464, 605-609 (2001).

[9] We can tell the pattern results from the anode grid rather than the cathode grid because the two grids are oriented at different angles.

[10] I. Haber, F.M. Bieniosek, C.M. Celata, et al., "End-to-End Simulation: The Front End," Laser and Particle Beams, (accepted for publication, 2002). 
[11] I. Haber, S. Bernal, C. M. Celata, A. Friedman, D. P. Grote, R.A. Kishek, B. Quinn, P.G. O'Shea, M. Reiser, J.-L. Vay, "Collective Space-Charge Phenomena in the Source Region," Proc. 2002 Charged Particle Optics Conf., submitted to NIM A (2002).

[12] S. Bernal, B. Beaudoin, Y. Cui, et al., "Intense beam transport experiments in a multi-bend system at the University of Maryland Electron Ring (UMER)," Proc. 2002 Charged Particle Optics Conf., submitted to NIM A.

[13] S. Bernal, R. A. Kishek, M. Reiser, and I. Haber, Phys. Rev. Lett., 82, 4002 (1999); S. Bernal, B. Quinn, P.G. O’Shea, M. Reiser, Physical Review ST-AB 5, 064202 (2002).

[14] R.A. Kishek, P.G. O'Shea, M. Reiser, Physical Review Letters 85, 4514 (2000).

[15] B.E. Carlsten and D.T. Palmer, Nucl. Instr. \& Meth. A 425, 37-50 (1999).

[16] H. Kandrup, I. Pogorelov, and I. Sideris, Mon. Not. R. Astron. Soc., 319, 43-62 (2000).

[17] R.A. Kishek, C. L. Bohn, I. Haber, P. G. O'Shea, M. Reiser, and H. Kandrup, Proc. 2001 Particle Accelerator Conf., Chicago, IL, ed. P. Lucas and S. Weber, I4 Cat. No. 01CH37268, p. 151 (2001).

[18] C.L. Bohn, in The Physics of High Brightness Beams, ed. Rosenzweig and Serafini, (World Scientific, Singapore: 2000), p. 358.

[19] T. Antonsen, Fan, E. Ott, and Garcia-Lopez, Phys. Fluids 8, 3094 (1996). 


\section{TABLES}

TABLE I. UMER Design Specifications

\begin{tabular}{lc}
\hline \hline Beam Energy & $10 \mathrm{keV}$ \\
$\beta(=\mathrm{v} / \mathrm{c})$ & 0.2 \\
Beam Current & $<=100 \mathrm{~mA}$ \\
Generalized perveance & 0.0015 \\
Emittance, 4x rms, norm & $10 \mu \mathrm{m}$ \\
Pulse Length & $40-100 \mathrm{~ns}$ \\
Ring Circumference & $11.52 \mathrm{~m}$ \\
Lap time & $197 \mathrm{~ns}$ \\
Pulse repetition rate & $60 \mathrm{~Hz}$ \\
Mean beam radius & $<=1 \mathrm{~cm}$ \\
FODO period & $0.32 \mathrm{~m}$ \\
0-current phase advance, $\sigma_{0}$ & $76^{\circ}$ \\
0-current Betatron tune, $v_{0}$ & 7.6 \\
Tune Depression & $>=0.2$ \\
\hline
\end{tabular}




\section{FIGURE CAPTIONS}

Fig. 1 Photographs of: (a) cathode (fine) and anode (coarse) grids without the beam; (b) beam incident on phosphor screen $6 \mathrm{~cm}$ downstream from anode grid, exhibiting a shadow of the anode grid; (c) simulation of Fig. $1 \mathrm{~b}$ using semi-Gaussian distribution with $12 \mu \mathrm{m}$ emit; and (d) same simulation with $4 \mu \mathrm{m}$ emit.

Fig. 2 Photograph of beam incident on a phosphor screen $104 \mathrm{~cm}$ downstream from anode while the five-beamlet mask is applied near the anode. The experiment is compared with the results of four simulations starting with a semi-Gaussian distribution at the anode, but with different initial emittances, marked on the figures.

Fig. 3 (a) Pepper-pot snapshot of phase-space at gun exit, taken with the phosphor screen $6 \mathrm{~cm}$ downstream from pepper-pot mask near anode and a cathode-grid biased at $45 \mathrm{~V}$; (b) simulation of pepper-pot starting with a annular-Gaussian distribution; and (c) simulation of grid shadow with annular-Gaussian (cf. Fig. 1b). Note that the pepper-pot holes are staggered in the experiment, but lie on a square grid pattern in the simulation.

Fig. 4 The annular-Gaussian velocity distribution: (a) the velocity distribution in a cross-section $f\left(v_{r}\right)$; (b) a projection of the initial distribution on $y-y^{\prime}$ trace space.

Fig 5 Photographs of beam cross-section along injector, at 33, 39, 50, 55, 61, 67, 71, and 103 $\mathrm{cm}$ from the anode plane: (a) experiment; (b) simulation with semi-Gaussian distribution; and (c) simulation with annular-Gaussian distribution.

Fig. 6 Evolution along the beamline of the natural logarithm of $x$ and $y$ "emittance moments" for 5 color-coded "clumps" of test particles launched in an initially anisotropic beam with $\varepsilon_{\mathrm{x}}$ 
$=2 \varepsilon_{y}$, and an average intensity $\chi=0.96$. The inset shows the same plot for an initially isotropic beam (i.e., $\varepsilon_{x}=\varepsilon_{y}$ ) with otherwise similar parameters.

Fig. 7 Trajectories of 20 test particles in $x-y$ space from one typical clump in (a) the isotropic beam; and (b) the anisotropic beam. 


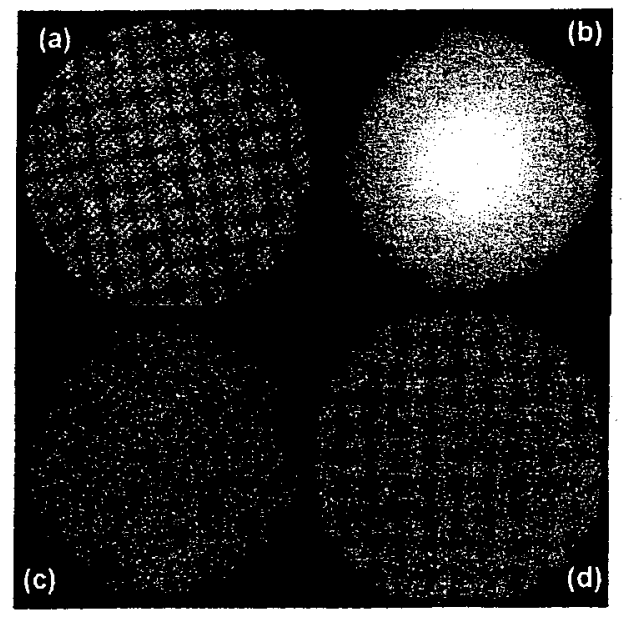




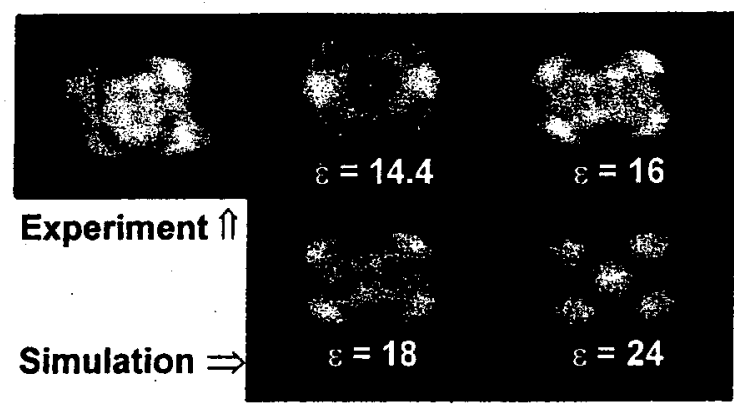




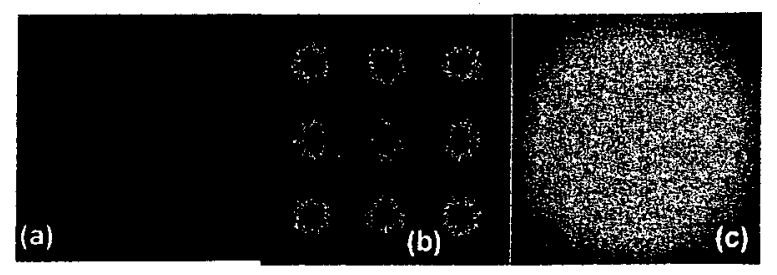

Kishek, et al.

Physics of Plasmas

Figure 3 


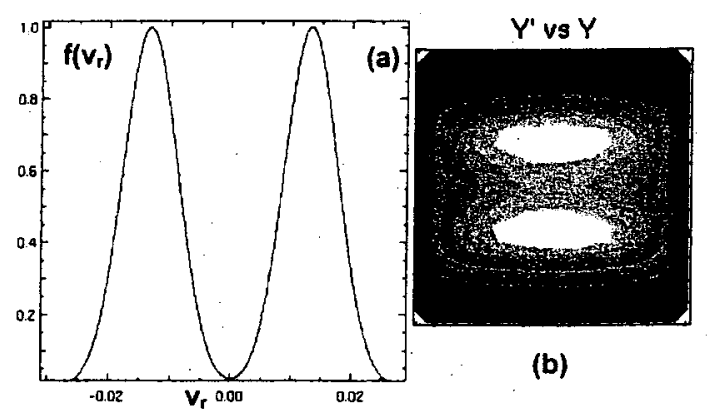




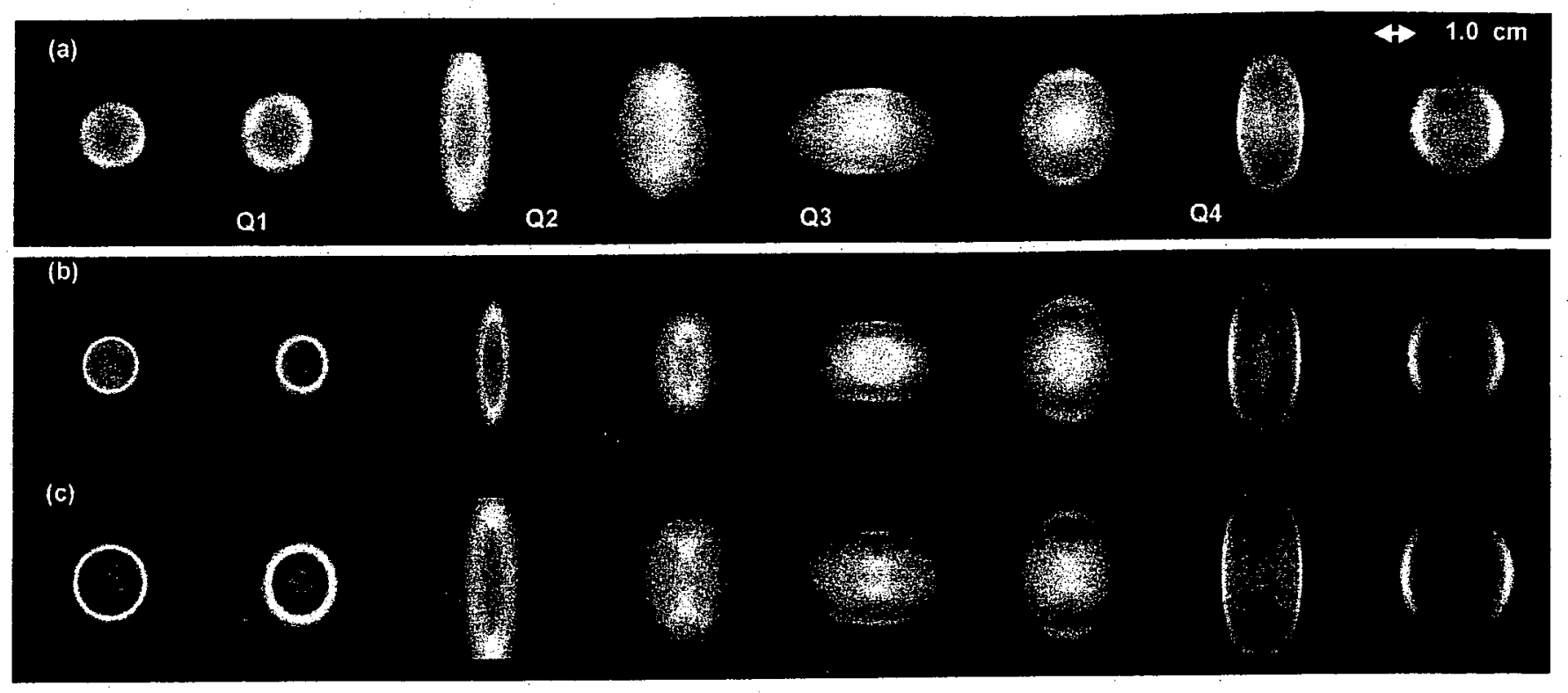




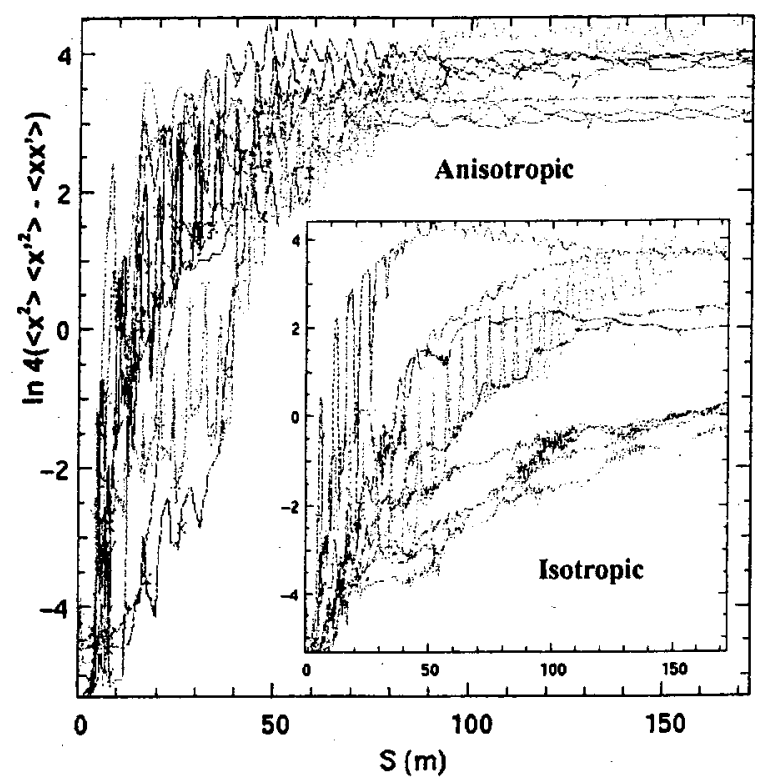




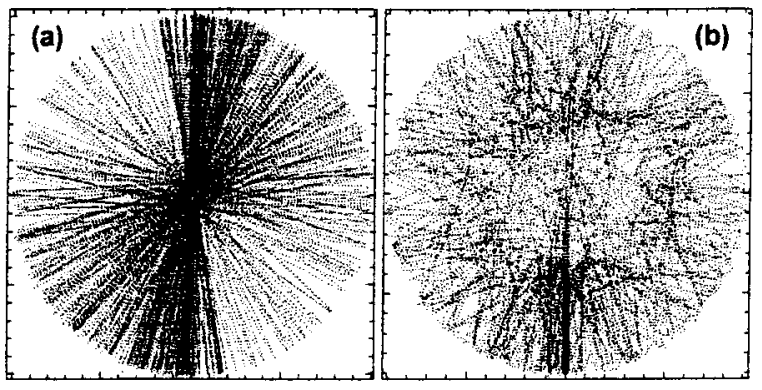




\title{
Intense beam transport experiments in a multi-bend system at the University of Maryland Electron Ring (UMER)
}

S. Bernal, ${ }^{1,2}$ B. Beaudoin, Y. Cui, M. Glanzer, T. F. Godlove, J. Harris, M. Holloway, I. Haber, R. A. Kishek, W-T. Lee, H. Li, D. Lamb, B. Quinn, M. Quirus, M. Reiser, A. Valfells, M. Walter, M. Wilson, R. Yun, Y. Zou, P. G. O'Shea

Institute for Research in Electronics and Applied Physics

University of Maryland

College Park, MD 20742

\begin{abstract}
We report on the results of beam transport experiments at the University of Maryland Electron Ring (UMER) over a distance of approximately four meters. The experiments involve a $10 \mathrm{keV}, 25-100 \mathrm{~mA}, 100 \mathrm{~ns}$ electron beam in a lattice consisting of one short solenoid, 24 printed-circuit magnetic quadrupole lenses, and a number of bending and steering dipole magnets. The diagnostics include capacitive beam-position monitors, phosphor screens, slit-wire emittance meters and others. We discuss the conditions required for matching the space-charge dominated beam into the UMER lattice, as well as emittance measurements and initial studies of longitudinal dynamics in the ring.
\end{abstract}

Key words: electron beam, magnetic quadrupole, space-charge, matching, emittance

PACS: $41.85 . \mathrm{Ja}, 29.27 . \mathrm{Eg}$

\footnotetext{
${ }^{\star *}$ Supported by the U.S. Department of Energy.

1 Tel. 301-405-7292

2 sabern@glue.umd.edu
} 


\section{Introduction}

The University of Maryland Electron Ring (UMER) is designed for scaled experiments employing low energy (up to $10 \mathrm{keV}$ ), high current (up to 100 $\mathrm{mA}$ ) electron beams [1]. With a strong-focusing (FODO) lattice consisting of 36 periods over a $11.52 \mathrm{~m}$ circumference (see Table 1), UMER can operate in a regime of unprecedented high beam intensity. Beam transport in this regime is dominated by space charge and is characterized by very low tune depression, i.e. the ratio of zero-current phase advances per period of the betatron oscillations with and without space charge [2]. As an example, the nominal $100 \mathrm{~mA}$ at $10 \mathrm{keV}$ in UMER models the beam physics of a $\mathrm{kA}$ beam of heavy-ions at $\mathrm{GeV}$ energies in a future heavy-ion fusion driver. Construction of a machine with a layout similar to UMER's was undertaken a few years ago at Lawrence Livermore National Laboratory. In the Livermore ring, a $80 \mathrm{keV}$ (initial energy) potassium-ion beam was successfully transported and accelerated over one quarter turn [3]. At the time of writing, UMER is also approaching completion of a one-quarter turn. Figures $1 \mathrm{a}$ and $1 \mathrm{~b}$ show the current experiment layout.

In the next section, we briefly describe the UMER electron gun. The main results from matching experiments in a straight section about one-meter long are summarized in Section 3. In Section 4, the first results of bend experiments over eight FODO periods, or $2.5 \mathrm{~m}$, approximately, are presented. The last section is devoted to conclusions.

\section{Electron Source and Beam Parameters}

The electron source is a Pierce-type gridded gun with variable perveance, similar to the gun described in [4]. The gun is cathode-driven, i.e. the cathode is pulsed to overcome the DC voltage applied to a control grid placed $0.15 \mathrm{~mm}$ in front of it. The gun operates in the $3-10 \mathrm{kV}, 1-100 \mathrm{~mA}$ ranges with current pulses around $100 \mathrm{~ns}$. The output current at a given energy can be varied by one of three methods or combinations thereof: by changing the anodecathode gap (over a $20 \mathrm{~mm}$ range), by adjusting the $\mathrm{DC}$ voltage applied to the control grid, or by means of a number of collimating apertures in front and near the anode. Of these methods, the latter is the preferred one for its simple implementation and mild effect on the output beam characteristics. The aperture plate also includes a pepper-pot for emittance measurements, and a five-beamlet mask for special studies of transverse beam dynamics. Measurements with the built-in pepper-pot have yielded a value of $60 \mu \mathrm{m}$ $\pm .10 \%$ for the $4 \mathrm{rms}$, unnormalized emittance about $5 \mathrm{~cm}$ from the aperture plate. A one-dimensional theory has been developed to understand the effect of 
the control grid on emittance growth (see Y. Zou et al. in these Proceedings). Furthermore, simulations of the gun have been undertaken (see I. Haber et al. in these Proceedings) in order to elucidate the initial beam distribution in phase space and understand its effects on beam evolution in UMER.

\section{Matching Experiments and Scaling}

Preliminary design calculations for any periodic focusing lattice can be based on the smooth approximation of the lattice [2]. In this approximation, the differential equations for the effective (2rms) envelope radii $X(z)$ and $Y(z)$ in the two transverse planes reduce to the algebraic equation for the "average" beam radius $R$ :

$$
k_{0}^{2} R-\frac{K}{R}-\frac{\varepsilon^{2}}{R^{3}}=0 .
$$

Here, $k_{0}=2 \pi / \lambda_{0}$ is the wavenumber, $\lambda_{0}$ is the wavelength of the transverse betatron oscillations without space charge. It can be written in terms of the zerocurrent phase advance $\sigma_{0}$ and the length $S$ of one lattice period as $k_{0}=\sigma_{0} / S$. Further, $K=2\left(I / I_{0}\right)\left(1 / \beta^{3} \gamma^{3}\right)$ is the generalized beam perveance, $I_{0}=17 \mathrm{kA}$, approximately, for electrons, $\beta=v / c, \gamma=\left(1-\beta^{2}\right)^{-1 / 2}(c$ is the speed of light, and $v$ is the particle's velocity), and $\epsilon_{x}=\epsilon_{y} \equiv \epsilon$ is the 4rms, unnormalized emittance. A convenient parameter, $u \equiv K S / 2 \sigma_{0} \varepsilon$, can be defined so the following expressions can be written for the tune depression and the average beam radius [2]:

$$
\frac{\sigma}{\sigma_{0}}=\sqrt{1+u^{2}}-u, \quad\left(\frac{R}{R_{0}}\right)^{2}=\sqrt{1+u^{2}}+u,
$$

where $R_{0}=\sqrt{\varepsilon / k_{0}}$ is the zero-current beam radius.

As mentioned above, the different currents in the UMER experiments are obtained mainly through the use of apertures. The aperture wheel is close to the beam waist near the electron gun anode, so we expect $K$, the generalized perveance, to be proportional to $R_{a}^{2}$, the aperture radius squared, and the effective emittance $\epsilon$ to be proportional to $R_{a}$. Therefore, the parameter $u$ in Eqs. (2) is directly proportional to $R_{a}$.

Thus, from the second of Eqs. (2), we have for the beam radius:

$$
\left(\frac{R}{R_{0}}\right)^{2} \rightarrow\left\{\begin{array}{crl}
2 u \propto R_{a}, & \text { if } u>>1, \\
1+u=1+\text { const } \cdot R_{a}, & \text { if } u<<1
\end{array}\right.
$$


Therefore, for space charge dominated beams the average size of the matched beam is proportional to the aperture size (recall that $R_{0}^{2}=\epsilon / k_{0}$, and $\epsilon \propto R_{a}$.) For emittance dominated beams, on the other hand, the average size of the matched beam is proportional to the square root of the aperture size. These results can also be obtained from Eq. (1).

Further, from the first of Eqs. (2), we have for the tune depression:

$$
\frac{\sigma}{\sigma_{0}} \rightarrow\left\{\begin{array}{cc}
1 / 2 u \propto 1 / R_{a}, & \text { if } u>>1 \\
1-u=1-\text { const } \cdot R_{a}, & \text { if } u<<1 .
\end{array}\right.
$$

Therefore, for space charge dominated beams the tune depression is inversely proportional to the aperture size. This is correct to within about $20 \%$ if $u>1.0$, or $\sigma / \sigma_{0}<0.4$.

The scaling expressed in Eqs. (3) and (4) was verified in initial transport experiments in UMER. These experiments, over a distance of about one meter, were restricted to the straight matching section consisting of a short solenoid and four printed-circuit magnetic quadrupoles [5]. Although the matching section is not a periodic lattice, the nature of the matching calculations with the envelope code SPOT [6]. (more specifically, the use of the "reference trajectory" as explained in [6]) assures that the crossing of the $\mathrm{X}$ and $\mathrm{Y}$ envelopes in the solution for the straight section is close to the average beam radius in the periodic FODO lattice. Three beam currents were used in the experiments, all at an energy of $10 \mathrm{keV}$ and all corresponding to a zero-current phase advance $\sigma_{0}=76^{\circ}$. Table 2 summarizes the beam parameters and Figures $2 \mathrm{a}-\mathrm{c}$ show phosphor screen pictures of the beam for all three experiments. The calculations with SPOT agree particularly well with the measured $2 \mathrm{rms}$ beam radii at the crossings, as shown in Figures $3 \mathrm{a}-3 \mathrm{c}$. Without a periodic lattice for checking true matching, the agreement at the crossing points was the main criterion for judging the matching calculations. Overall discrepancies between the calculated envelope and the experiment can be explained by uncertainties in the solenoid field, emittance and beam initial conditions, and, to a lesser degree, errors in image processing (e.g. mm/pixel calibration).

From Figs. 2 and 3 (notice the difference in scales in both sets of figures) it is clear that the "average" beam radius is inversely proportional to the tune depression. This is expected to be a good approximation for the range of values of the $u$ parameter in the experiments (see Table 2). The results also validate the beam parameters at $z=0$ (the aperture plate), $R_{0}=3.2 \mathrm{~mm}$, and $R_{0}^{\prime}=0$, which were determined before from studies of the free expansion of the electron beam. Also from Fig. 2, the internal structure of the beam is different for the three currents. Issues related to the detailed evolution of the particle distribution, however, will be addressed in another publication. It is 
worth adding, nonetheless, that the results should help dispel a widespread misconception about beam dynamics: that an aperture does not affect the beam significantly since the core's particle density is left unchanged. While this may be an accurate statement for low-intensity beams, it does not hold in general. As we have seen, an aperture changes the relative contributions of thermal and space charge effects by changing the ratio of emittance to generalized beam perveance. As a consequence, both the transverse density and velocity profiles are affected.

Emittance measurements were conducted using the pepper-pot in a diagnostics chamber connected at the end of the straight section. The plane of the pepperpot was $1.2 \mathrm{~m}$ from the aperture plate, and pictures were taken with a phosphor screen placed $6.5 \mathrm{~cm}$ from the pepperpot. Since the distance from the last quadrupole (Q4) to the pepperpot plane was rather large, the strength of all focusing elements were adjusted to obtain a reasonable beam size at the pepperpot. The measured 4rms, unnormalized emittance of the $100 \mathrm{~mA}, 10$ $\mathrm{keV}$ beam was $100 \mu \mathrm{m} \pm 15 \%$, indicating a $60 \%$ increase over the emittance measured at the output of the electron gun in a previous set of experiments. However, mechanical constraints prevented us from repeating the emittance measurements near the gun output to confirm the emittance increase. A computer simulation study [7] of the imaging of a five-hole mask as a function of initial beam emittance (of the full beam behind the mask) points to an emittance value of $80-90 \mu \mathrm{m}$.

\section{Bend Experiments}

Following the straight section, a pipe with a 10 degree bend part was added. The new section (see Fig. 1b) accommodates three additional magnetic quadrupoles before the (DC) injection bend dipole, and one quadrupole after the bend which constitutes the first ring quadrupole (QR1). The four $20^{\circ}$ bend sections contain four quadrupoles and two bend dipoles each. All magnets, except for the short solenoid before Q1, are made of flexible printed-circuits (PC) and have been extensively characterized [8]. In addition to the elements shown in Fig. $1 \mathrm{~b}$, a number of PC short steering dipoles and Helmholtz coils are employed. The latter are used to compensate for the Earth's magnetic field.

Assuming complete shielding of the Earth's magnetic field, the required current in the main dipole magnets is $3.0 \mathrm{~A}$. This is the current for a $10^{\circ}$ bend of a $10 \mathrm{keV}$ electron over the effective length of the magnet. However, the beam in UMER circulates in a direction such that the Earth's field helps in deflecting it through the main ring sections. Thus, the required bend dipole current is reduced from $3.0 \mathrm{~A}$ to $2.35 \mathrm{~A}$, which alleviates significantly power consumption and heating of the magnets. The action of the Earth's field over the match- 
ing/injection section is not desirable, however, so two sets of Helmholtz coils are used: one set over the straight section from behind the solenoid to Q5 is oriented to balance the net Earth's B-field; a second set of coils over the bend tube, from Q5 to QR1 in Fig. 1b, compensates for the vertical component of the Earth's field. Since the DC injection dipole, D0, is in the latter region, the required current for injection into the ring is close to the expected $3.0 \mathrm{~A}$. Without the Helmholtz coils over the bend tube, however, the required current for D0 is too high, around 3.6 A. Furthermore, the horizontal component of the Earth's field deflects the beam in a vertical direction, so additional correctors are placed in each bend section.

The main diagnostics at this stage in the project are fluorescent screens ( $\mathrm{P}-43$ phosphor coating). The $3.8 \mathrm{~cm}$ (dia.) screens are placed in the two chambers in the matching/injection section (IC1 and IC2 in Fig. 1b), and in each of the four bend sections (RC1-RC4), and at the end of the system. The phosphorscreen image of the beam is reflected by a single-surface mirror placed at $45^{\circ}$ to the screen so the beam can be monitored through a window. A standard CCD camera and associated hardware and software for video capture and processing are used. Current measurements are carried out with a fast Pearson transformer located between Q2 and Q3. Furthermore, fast capacitive beam position monitors, or BPMs for short, [9], are also employed in all chambers except IC1 and the end chamber. The BPMs are used to help in alignment and also for studies of current waveform evolution (longitudinal dynamics). Other diagnostics still under testing include a slit-wire emittance meter and an energy analyzer.

Successful transport experiments in the bend system, i.e. experiments with no beam losses or major beam distortions, depended on a combination of factors: mechanical alignment, small steering corrections as dictated by the diagnostics (phosphor screens and BPMs), first-order matching calculations, and balancing of the Earth's B-field over the matching/injection sections. An optical alignment system was used to lay bulk components of the system such as bend sections, support plates and the end diagnostics chamber. Of particular importance was assuring that all support plates shared a common plane: the quadrupoles and bending dipoles are rigidly attached to the support plate so roll errors are minimized by leveling these plates individually. Furthermore, the manufacturing and assembly of quadrupole and dipole magnet mounts were subject to errors. The magnetic axes of quadrupoles can be determined to within $\pm 0.25 \mathrm{~mm}$, so this figure represents a lower bound on alignment tolerance. Finally, inaccurate welding of parts of the BPM chambers and errors in the bent pipes were compensated for in the bellows at the downstream end of each ring chamber.

With unavoidable mechanical errors, beam steering becomes important. In UMER, the short steering dipoles are made of flexible printed-circuits as the 
main magnets. With a peak field of $0.61 \mathrm{G} / \mathrm{A}$ (calculated and measured) and an effective length of $5.3 \mathrm{~cm}$, the steering dipoles have a "deflecting power" of $10 \mathrm{mrad} / \mathrm{A}$ for $10 \mathrm{keV}$ electrons. Interestingly, the $\mathrm{PC}$ quadrupoles have a dipole component with about one-half the "deflecting power" (4.5 mrad/A) if a beam centroid offset of $0.1 \mathrm{~cm}$ is assumed. This is so for typical operating currents of $1 \mathrm{~A}$ and $2 \mathrm{~A}$ for the steering dipoles and $\mathrm{PC}$ quadrupoles, respectively.

Another important factor in the experiments is the rms envelope matching calculation. The initial calculations employed the envelope code SPOT [6], which is based on a system without bending magnets. The beam envelope is matched to $\mathrm{PQ}$, the quadrupole following $\mathrm{Q} 6$ and right before the bend dipole D0 (Fig. 1b). The nominal zero-current phase advance is $\sigma_{0}=76^{\circ}$ in both transverse planes. Figures $4 \mathrm{a}-\mathrm{b}$ show phosphor screen pictures of the beam in all six BPM chambers illustrated in Fig. 1b. Two cases are shown: $100 \mathrm{~mA}$ (full beam) and $25 \mathrm{~mA}$ (using a $1.5 \mathrm{~mm}$ radius aperture and an anode-cathode gap that is slightly different from the one used for the experiments in the straight section). The size of the $25 \mathrm{~mA}$ beam is in most cases about one-half the corresponding beam size of the full beam, revealing the same relationship between beam size and tune depression found before in the straight section experiments (see Section 3). The beam distortions of the full beam are most likely produced by image forces in the bend tube; these are difficult to correct because of the lack of diagnostics in that section. It must be noted, though, that the beam ellipse is vertical at the plane of the injection bend (D0 in Fig. 1b) to avoid the compound problem of bending and image forces in that plane. To accomplish this beam orientation, the quadrupole polarities in the bend experiments had to be reversed relative to the polarities in the straight section experiments.

In addition, the envelope calculation vs. experiment for the $100 \mathrm{~mA}$ case is shown in Figure 5. As seen in the figure, the beam is confined within the calculated envelope. However, the broad envelope oscillation in the plot suggests a degree of overall envelope mismatch. Similar calculations for the $25 \mathrm{~mA}$ reveal larger discrepancies between calculated envelope and experiment. This is especially so at RC3 (Fig. 4b) where the effective vertical beam dimension is too large, even if the observed (and unexpected) halo is artificially masked for the computation. Experiments are in progress to check refined calculations that include bending (see $\mathrm{H}$. $\mathrm{Li}$ et al in these Proceedings) and to measure emittance at the end of the system.

Finally, the first measurements of longitudinal dynamics effects in UMER were conducted at this phase of the project. Comparison of signals from the BPMs at chambers IC2 and RC4 shows that the total beam length increases by $7 \%$. The theoretical estimate, based on a one-dimensional cold fluid model [10], predicts an increase of $8-10 \%$ over the $3.0 \mathrm{~m}$ that separates the chambers. 
Future experiments in UMER will involve longitudinal beam focusing with a number of induction gaps in the lattice [1].

\section{Conclusions}

Current experiments at the University of Maryland Electron Ring (UMER) use a matching/injection section and eight FODO full periods for a total length of nearly four meters from the output of the electron gun. Experiments with different currents in the straight section following the electron gun show that the average beam size is approximately proportional to the inverse of the tune depression, in agreement with predictions from the smooth approximation of the lattice. Further, transport experiments with the full beam over a net bend of $70^{\circ}$ are made possible by careful mechanical alignment, small steering corrections, first-order matching calculations, and balancing of the Earth's B-field in the matching/injection section. Refinement of the matching calculations and additional measurements of beam emittance and energy spread will be done before moving to the next phase. The new phase will involve experiments over an additional four to six FODO periods.

\section{Acknowledgments}

We thank D. Feldman for the picture of the UMER experiment, and D. Brosius for invaluable help with LateX.

\section{References}

[1] O'Shea et al, Nucl. Instrum. Phys. Res. A 464, 646-652 (2001).

[2] M. Reiser, Theory and Design of Charged-Particle Beams, Wiley \& Son, New York (1994), and references therein.

[3] L. Ahle et al, Nucl. Instrum. Phys. Res. A 464, 557-562 (2001).

[4] J.G. Wang, E. Boggasch, P. Haldemann, D. Kehne, M. Reiser, T. Shea, and D.X. Wang, IEEE Trans. Elec. Dev., 37, 12, (1990) 2622.

[5] S. Bernal et al, Proceedings of the 2001 Particle Accelerator Conference, Chicago, Ill (IEEE, Piscataway, NJ, 2001), p. 2129.

[6] C. K. Allen, S.K. Guharay and M. Reiser, in Proceedings of the 1995 Particle Accelerator Conference, Dallas, Texas (IEEE, New York, 1996), p. 2324. 
[7] I. Haber et al. To appear in Laser and Particle Beams (2002), 20, 1-3.

[8] W.W. Zhang et al, Phys. Rev. ST Accel. Beams 3, 122401 (2000).

[9] J. Harris et al, Proceedings of the 2001 Particle Accelerator Conference, Chicago, Ill (IEEE, Piscataway, NJ, 2001), p. 1387.

[10] Dunxiong Wang, $\mathrm{Ph} . \mathrm{D}$. Thesis, Electrical Engineering Department, University of Maryland, College Park, 1993. 


\begin{tabular}{|l|l|}
\hline \multicolumn{2}{|l|}{ Table 1. Beam and Lattice Parameters in UMER } \\
\hline Beam Current & $100 \mathrm{~mA}$ \\
Beam Energy, $\beta(=v / c)$ & $10 \mathrm{KeV}, 0.2$ \\
Beam Perveance, $K$ & $>0.0015$ \\
Emittance, $4 \mathrm{rms}$ unnorm., $\varepsilon$ & $60 \mu \mathrm{m}$ \\
FODO period, $S$ & $0.32 \mathrm{~m}$ \\
Quadrupole effective length, $l$ & $0.0363 \mathrm{~m}$ \\
Tune Depression & $>0.12$ \\
\hline
\end{tabular}

Table 2. Beam Parameters in Straight Section Experiments

\begin{tabular}{|l|c|c|c|c|}
\hline & $\begin{array}{c}\text { at } 10 \mathrm{keV}, \sigma_{0}=\mathbf{7 6}^{\mathbf{0}} \\
(\mathrm{mA})\end{array}$ & $\begin{array}{c}\text { Emittance } \\
(\mu \mathrm{m})\end{array}$ & $\begin{array}{c}\text { Avg. Beam Radius } \\
(\mathrm{mm})\end{array}$ & $\begin{array}{c}\text { Tune Depression, } \\
\text { and } u=K S / 2 \sigma_{0} \varepsilon\end{array}$ \\
\hline Full Beam & $100 \pm 5 \%$ & $60 \pm 10 \%$ & 10.3 & $0.15,3.0$ \\
Beam 2 & 21 & 30 & 4.95 & $0.35,1.3$ \\
Beam 3 & 9.0 & 25 & 3.75 & $0.54,0.65$ \\
\hline
\end{tabular}




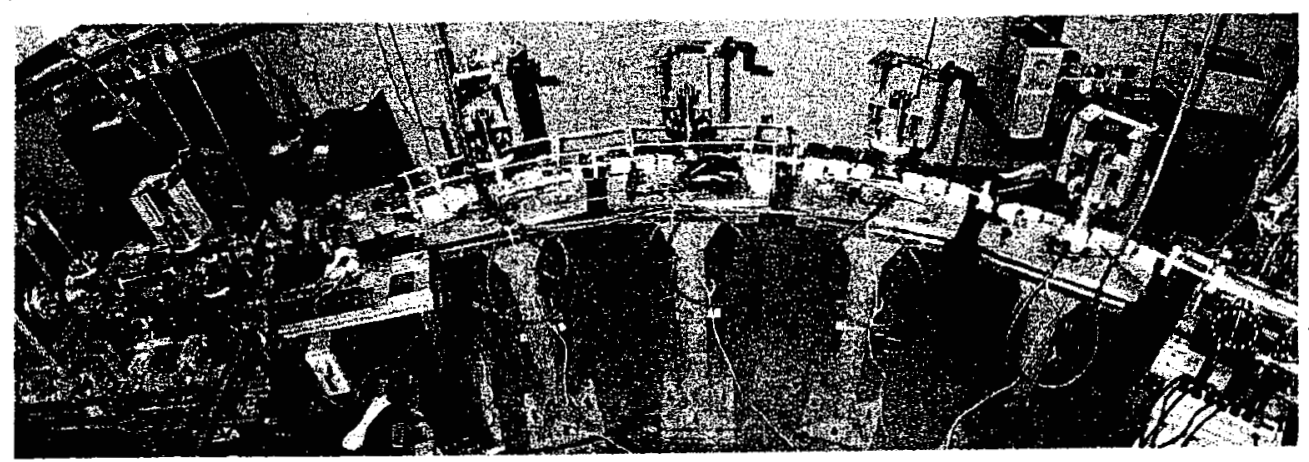

Fig. 1(a). Photograph of 70 deg. bend setup in UMER.

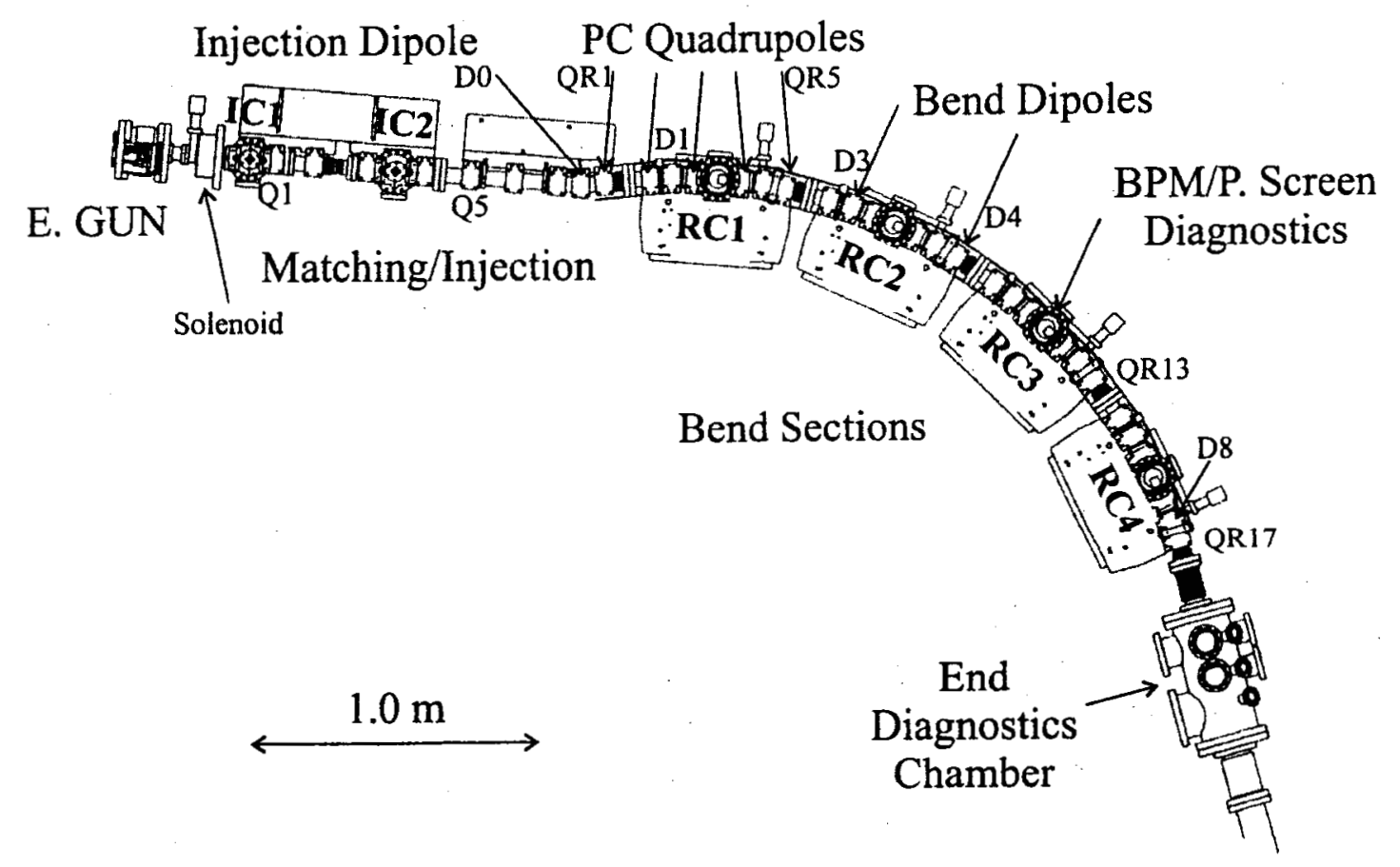

Fig. 1(b). Schematics of 70 deg. bend setup in UMER. "BPM" stands for "beam position monitor", and "P. Screen" for "Phosphor Screen". 

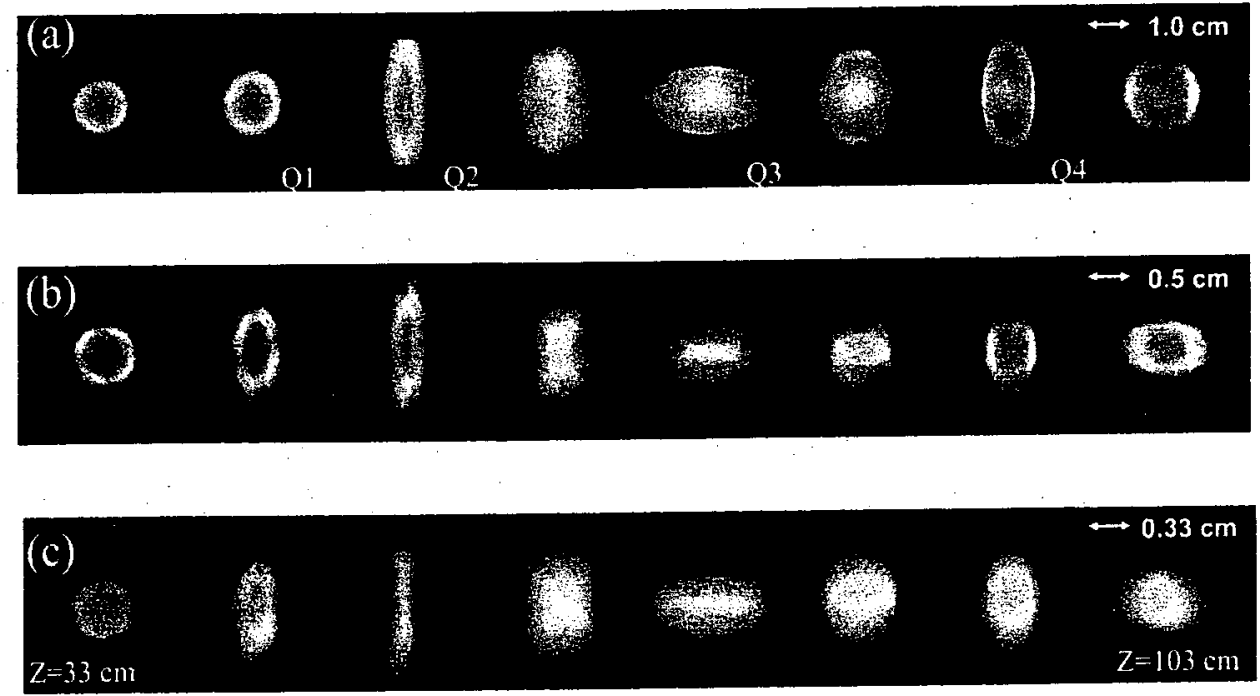

Fig. 2. Partial sample of phosphor screen pictures in straight-section experiments for: (a) Full electron beam (100 mA), (b) apertured beam (21 mA) and (c) 9.0 $\mathrm{mA}$ beam. Notice the difference in scales. The aperture plate near the electron gun output defines the plane $z=0$. 

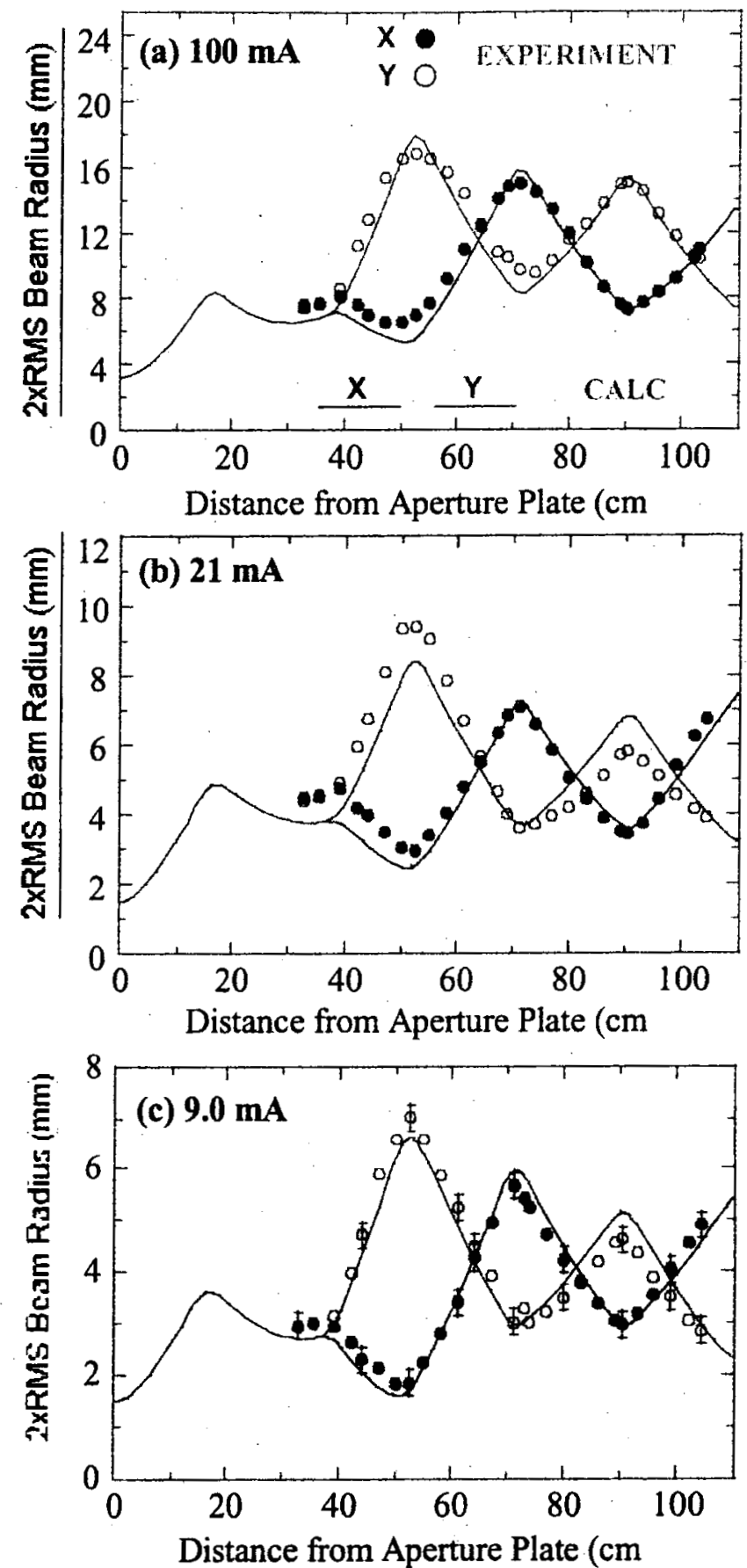

Fig. 3. Calculations of effective $K-V$ envelope radius vs. $2 \mathrm{rms}$ beam radius from experiment (see Fig. 2) for: (a) Full beam (100 mA), (b) apertured beam (21 mA) and (c) $9.0 \mathrm{~mA}$ beam. Notice the difference in scales. 


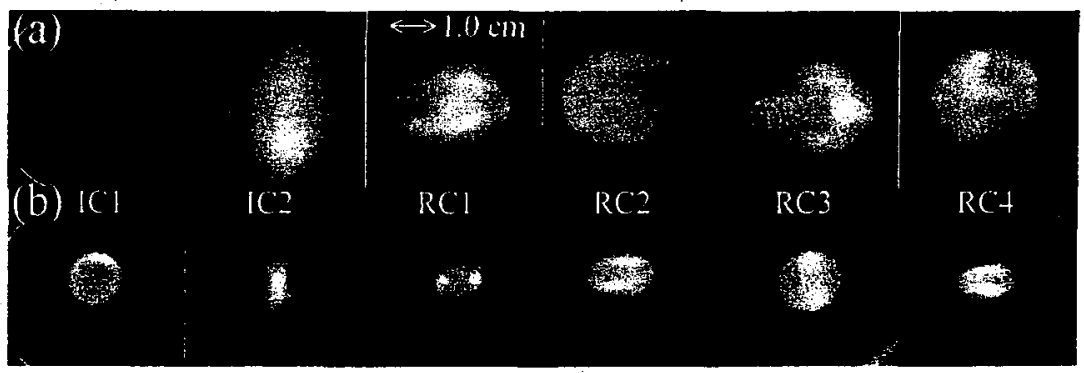

Fig. 4. Fluorescent screen pictures in bend experiments at $10 \mathrm{keV}$ with: (a) Full electron beam ( $100 \mathrm{~mA}$ ), and (b) $25 \mathrm{~mA}$ beam. The labels indicate the diagnostics chambers as illustrated in Fig. $1 \mathrm{~b}$. 


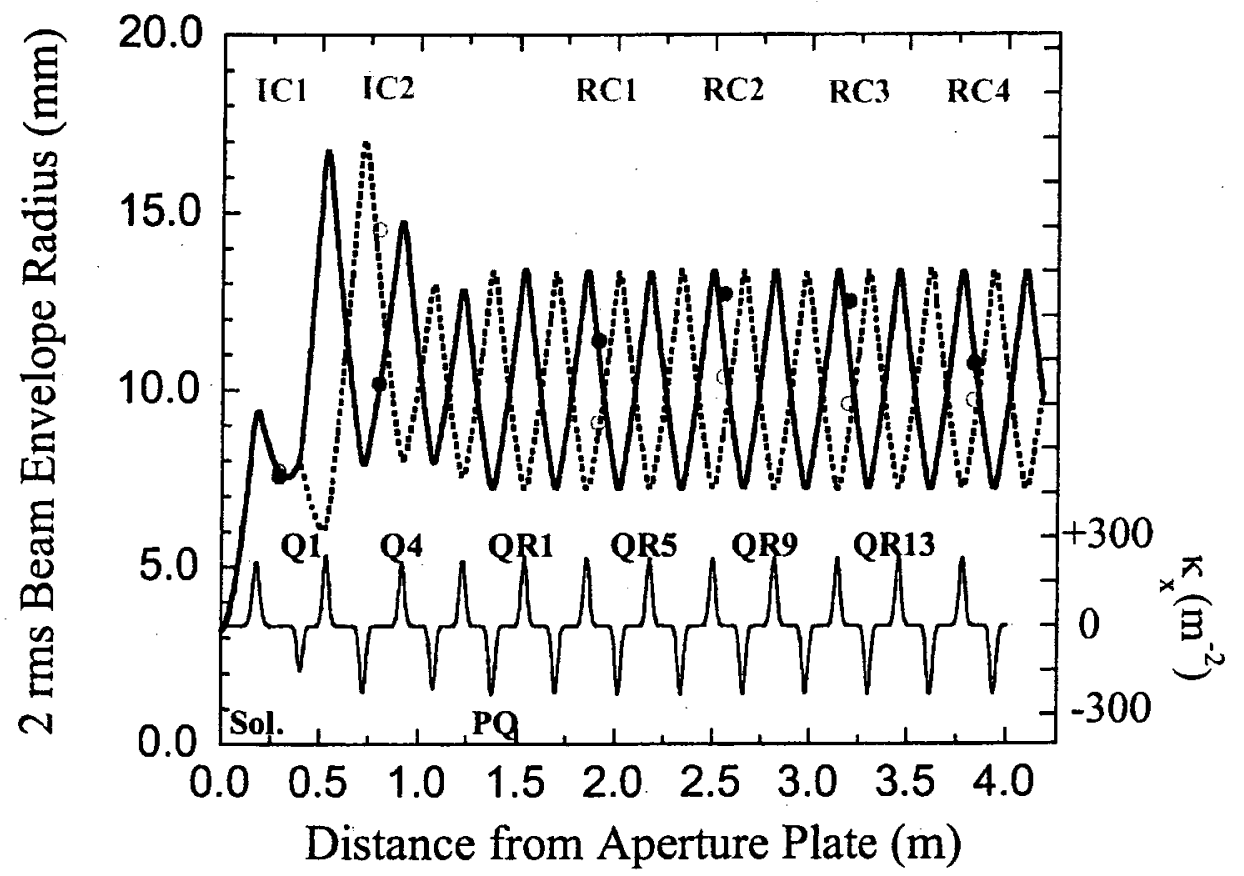

Fig. 5. K-V envelope calculation (no bends included) vs. experimental beam radii (top), and horizontal focusing function $\kappa_{x}(z)$ (bottom). The solid curve on top represents the $X$-envelope. Positive focusing function indicates focusing in the $x$ plane, i.e the horizontal plane. 


\title{
COMPUTER SIMULATION OF THE UMER ELECTRON GUN*
}

\author{
I. Haber, Plasma Physics Division, Naval Research Laboratory, Washington, DC 20375
}

\author{
S. Bernal, R. A Kishek, P. G. O'Shea, M. Reiser, A. Valfells \\ Institute for Research in Electronics and Applied Physics, \\ University of Maryland, College Park MD 20742 \\ D. P. Grote, Heavy Ion Fusion - Virtual National Laboratory \\ Lawrence Berkeley National Laboratory, Berkeley, CA 94720
}

\begin{abstract}
An important feature of the UMER experimental program $[1,2]$ is the continuing use of comparison between computer simulation and experimental measurement. These comparisons have, for example, explained several significant features observed in the prototype injector experiment. Therefore, during assembly and testing of the UMER injector two- and threedimensional simulations are being conducted with the aim of understanding the space-charge-dominated physics in the gun and injector transport sections, as well as interpreting experimental data. These simulations are important to providing a realistic initial beam distribution for simulating beam dynamics in the main ring. Emphasis here is on simulating the beam propagation in the gun region between the cathode grid and the anode grid for comparison with measurements at the exit from the gun structure.
\end{abstract}

\section{INTRODUCTION}

The University of Maryland Electron Ring (UMER) $[1,2]$ has been designed to examine the physics of a highly space-charge-dominated electron beam as it propagates through hundreds of periods in an alternatinggradient transport system. Because this experimental apparatus can access a range of beam parameters not heretofore accessible, considerable effort has been expended in the careful design of a comprehensive set of beam diagnostics so that the beam can be accurately characterized and the physics clearly understood. Because of the complexity of the nonlinear physics expected to be of importance in a beam which is so highly space-charge-dominated, simulations have been a primary tool in designing the experiment and are expected to play a major role in interpreting the measured experimental data.
Many of the collective phenomena important to understanding the evolution of a space-charge-dominated beam have been found to depend on the detailed characteristics of the distribution function.[3] Excellent agreement was therefore obtained using the prototype injector for recent simulation-experiment comparisons.[4] The well-characterized initial distribution was created by passing the beam through an aperture that excluded all but the uniform central portion of the beam

Excellent agreement between simulation and experiment was also obtained in a previous [5] experiment where the beam was masked into five beamlet, which were then allowed to merge and form images of the original configuration. However, in this experiment, difficulty was encountered in attempts to incorporate an initially observed hollowing in the beam profile. This was attributed to inadequate knowledge of internal correlations in the beam distribution.

The possible presence of internal correlations, which are difficult to measure, can limit adequate prediction of the evolution of a space-charge-dominated beam. A simple example of this behavior would be the reconstruction of the distribution of an axisymmetric beam from $x$ and $y$ phase-space measurements. Though identical measurements in the two planes would strongly suggest axismmetry, there are other distributions that would also be consistent with the measured profile. Because of the importance of the initial distribution function to predicting beam evolution in UMER, it is important to begin simulations at the emitter surface, which is likely to be free of any significant internal correlations. "First principles" simulation of the beam starting from the cathode should then capture the beam dynamics, including any correlations that develop as the beam evolves.

\footnotetext{
Work supported by the U. S. Department of Energy at NRL under contracts DE-AI02-93ER40799 and DE-AI02-94ER54232, at U. Md. under DE-FG02-92ER54178 and DE-FG02-94ER40855, and at the HIFVNL under DE-AC03-76SF00098 and W-7405-Eng-48.
} 


\section{SIMULATION METHODS}

Although it is desirable to conduct "first principles" simulations of the beam evolution beginning at the cathode surface, such a brute force simulation would be a very ambitious undertaking. A primary obstacle is the gun geometry, which has a grid $0.15 \mathrm{~mm}$ away from the cathode surface, and spacing of $0.15 \mathrm{~mm}$ between the grid wires. The complex geometry in the cathode-grid region can therefore substantially influence the characteristics of the emerging beam. Since this distance is much smaller than the cathode to anode distance, which is measured in centimeters, the disparate scales make it difficult to treat both regions accurately in a single simulation. Also complicating experiment/simulation comparisons is the difficulty of accurately measuring the grid potential and current when the beam is turned on because the grid floats at a $10 \mathrm{kV}$ potential.

It is also important to understand the time-dependent behavior of the gun structure, in order to simulate experiments on the longitudinal beam dynamics, since transients in the gun can be on the same time scale as the longitudinal phenomena to be measured. The simulations are therefore being performed using the combined plasma accelerator WARP code, [6] which is a fully self-consistent time dependent treatment. To make the runs tractable, the beam dynamics in the cathode-to-grid region are being treated separately from the cathode-to-anode region. The simulations discussed here have, to date, only examined the second region. The results should therefore be considered descriptive rather than predictive, since feedback between the two regions could change the detailed behavior.

A specified beam current and emittance is therefore injected into this region with a nominal 20 volt energy, which approximates the expected value for the actual grid-cathode voltage. However, the beam behavior has been found somewhat insensitive to the value of this voltage in the regime of gun operation. Parameters are then varied to understand the range of possible operating conditions.

To further reduce the numerical requirements, the threedimensional fields of the entire gun structure are solved and saved. The potential on a cylinder outside the beam is then applied as the boundary of a second simulation so that only the self-consistent field within this smaller region must be calculated. It was found that the added potential at the bounding surface from the presence of the beam is much less than 1 percent.

\section{SIMULATION RESULTS}

The gun configuration used in UMER, which employs a cathode grid to rapidly turn on the beam current, operates in a manner similar to the power amplification mode of a traditional vacuum triode.[7] Because of the need to understand details of the beam evolution as it is injected into the UMER ring, it is necessary to accurately characterize the beam phase space distribution. This is in contrast to merely describing the output current characteristics as has usually been done when describing operation of a vacuum triode.

One area of significance that will not be discussed here, is the modification to the beam characteristics that results from the geometric complexity in the region between the cathode and the cathode grid. Since the separation between the wires of the cathode grid is comparable to the distance from the emitting surface, both the longitudinal and transverse velocity distributions can be a complex function of the voltage applied to the grid structure. In view of the difficulty in measuring the actual grid to cathode potential, study of this behavior has been deferred pending a better understanding of the beam dynamics in the downstream region.

The simulations discussed here therefore assume that the beam is injected into the simulated region with a specified spatially uniform current and the idealized longitudinal and transverse velocities are assumed to be a Gaussian distribution emerging from the $-0.1 \mathrm{eV}$ cathode. A cathode-grid to anode-grid distance of $220 \mathrm{~mm}$ was assumed as typical of gun operation. This distance can, in fact, be mechanically varied to adjust the gun perveance. With the latest upgrade to the pulser circuit, the gun was found to actually draw more than the nominally desired $138 \mathrm{~mA}$ which corresponds to the desired $120 \mathrm{~mA}$ nominal exit current at $10 \mathrm{kV}$, adjusted for the $87 \%$ transparency of the anode grid.

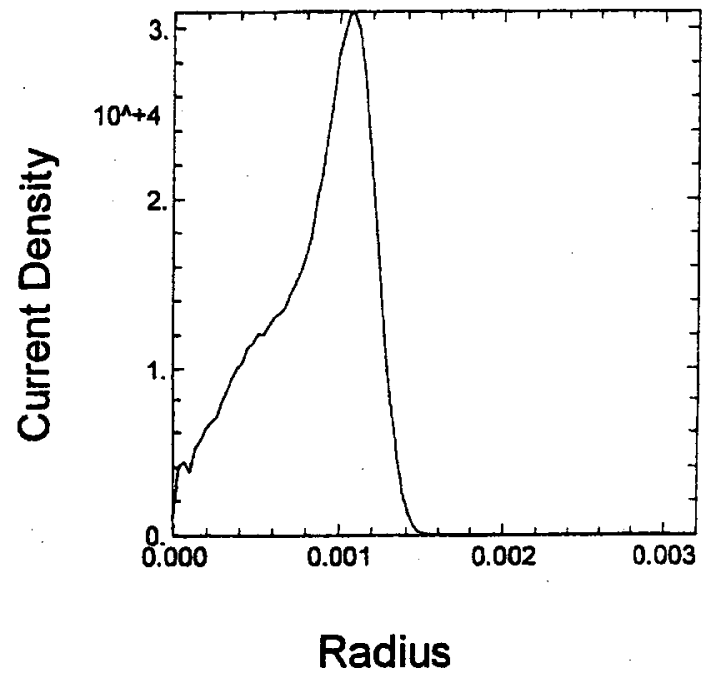

Fig. 1. Radial variation of the current density (in arbitrary units) at the plane of the anode grid, for an injected current of $138 \mathrm{~mA}$.

Figure 1 is a plot of the radial variation in current density for the nominal injected current of $138 \mathrm{~mA}$. At this current level, the beam is quite hollow. An interesting characteristic of the beam distribution is shown in Fig. 2, which is a plot of the rms thermal velocity as a function of radius. Because the orbits in the diode region are close to laminar, the truncation of a fluid moment expansion by neglecting the heat flow term can be used to predict a 
number of collective space-charge phenomena.[8] In the current case this approximation suggests that the beam in the diode region should approximately obey a local adiabatic approximation, so that the temperature as a function of radius should be proportional to the square root of the current density.

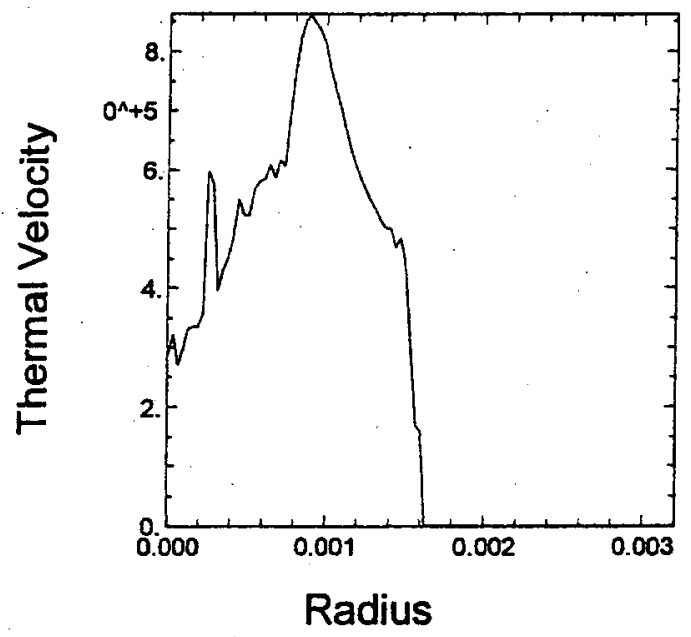

Fig. 2. Radial variation in rms velocity for the $138 \mathrm{~mA}$ beam plotted in Fig. 1.

The injected current was chosen with a degree of arbitrariness. Because the actually observed current in the diode under these conditions was somewhat greater than $138 \mathrm{~mA}$, a simulation was performed with the current increased by $50 \%$. The hollowing in current density across the beam is then somewhat reduced as shown in the plot in Fig. 3. Increasing the current to twice the initial run or $276 \mathrm{~mA}$ further decreases the observed hollowing so that the beam at the anode grid is almost uniform.

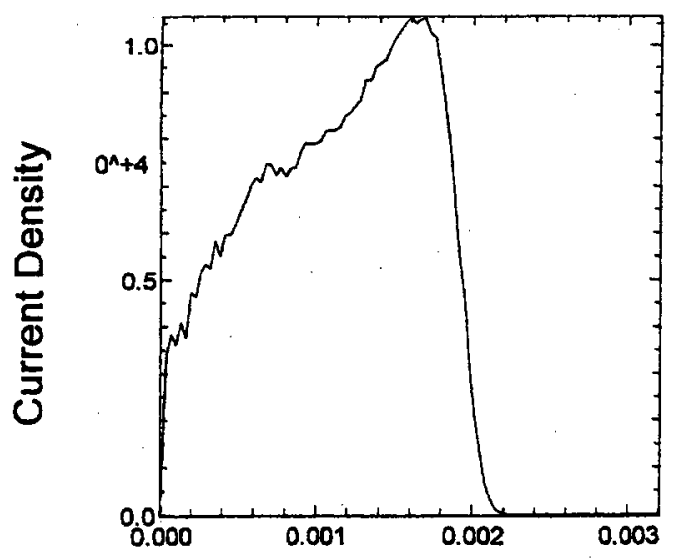

Radius

Fig 3. Radial variation in current density when the injected current is increased $50 \%$ to $207 \mathrm{~mA}$.
The simulations can also be used to examine the timedependent behavior of the beam. An interesting characteristic of the beam head is observed when the beam is turned on instantaneously at the cathode grid. The current reaching the anode still has an approximately. 1 ns rise time which results from the central portion of the current pulse reaching the anode before the outer edges.

\section{CONCLUSIONS}

Preliminary simulations have been performed to examine beam characteristics in the region between the cathode grid and the anode grid in the UMER gun. The observed variability in the cross section as the current is varied should be useful, in concert with measurement, in characterizing details in the beam distribution important to predicting the downstream evolution, but difficult to obtain with simulation or measurement alone. Further work to characterize the beam behavior in the $0.15 \mathrm{~mm}$ region between the cathode and the cathode grid should assist in further refining this procedure and eventually approaching the ability to do "first-principles" predictions of the beam characteristics including the details of the three-dimensional phase space characteristics.

\section{ACKNOWLEDGEMENT}

The authors would like to thank David Kehne of FM technologies for his assistance.

\section{REFERENCES}

[1] P. O'Shea, et al. "The University of Maryland Electron Ring (UMER)," invited presentation at this conference.

[2] http://www.ireap.umd.edu/umer

[3] 1. Haber, A. Friedman, D. P. Grote, S. M. Lund, R. A. Kishek, "Recent Progress in the Simulation of Heavy Ion Beams," Phys. Plasmas, 6, 2254 (May 1999).

[4] S. Bernal, R. A. Kishek, M. Reiser, and I. Haber, "Observations and simulations of Transverse Density Waves in a Collimated Space-Charge Dominated Electron Beam," Phys. Rev. Lett., 82, 20, 4002. (17 May 1999).

[5] I. Haber, D. Kehne, M. Reiser, and H. Rudd, "Experimental, Theoretical, and Numerical Investigation of the Homogenization of Density Nonuniformities in the Periodic Transport of a SpaceCharge Dominated Beam," Phys Rev A, 44, 5194 (Oct. 15, 1991).

[0] D. P. Grote, A. Friedman, I. Haber, W. Fawley, and J.L. Vay, New Developments in WARP: Progress Toward End-to-End Simulation," Nucl. Instr and Methods in Phys. Res. A 415 428-432 (1998).

[7] Karl. R. Spangenberg, Vacuum Tubes, (McGraw Hill, NY, 1948).

[8] Steven M. Lund, and Ronald C Davidson, "Warm Fluid Description of Intense Beam Equilibrium and electrostatic Stability Properties," Phys. Plasmas, 5, 3028 (Aug. 1998). 


\title{
End-to-end simulation: The front end
}

\author{
I. HABER, ${ }^{1,3}$ F.M. BIENIOSEK, ${ }^{2}$ C.M. CELATA, ${ }^{2}$ A. FRIEDMAN, ${ }^{2}$ D.P. GROTE, ${ }^{2}$ \\ E. HENESTROZA, ${ }^{2}$ J.-L. VAY, ${ }^{2}$ S. BERNAL, ${ }^{3}$ R.A. KISHEK, ${ }^{3}$ P.G. O'SHEA, ${ }^{3}$ \\ M. REISER, ${ }^{3}$ AND W.B. HERRMANNSFELDT ${ }^{4}$ \\ 'Icarus Research Inc., Bethesda, MD 20824-0780, USA \\ ${ }^{2}$ Heavy Ion Fusion-Virtual National Laboratory, Berkeley, CA 97420, USA \\ ${ }^{3}$ Institute for Research in Electronics and Applied Physics, University of Maryland, College Park, MD 20742-3511, USA \\ ${ }^{4}$ Stanford Linear Accelerator Center, Stanford University, Stanford, CA 94305, USA
}

\begin{abstract}
For the intense beams in heavy ion fusion accelerators, details of the beam distribution as it emerges from the source region can determine the beam behavior well downstream. This occurs because collective space-charge modes excited as the beam is born remain undamped for many focusing periods. Traditional studies of the source region in particle beam systems have emphasized the behavior of averaged beam characteristics, such as total current, rms beam size, or emittance, rather than the details of the full beam distribution function that are necessary to predict the excitation of the collective modes. Simulations of the beam in the source region and comparisons to experimental measurements at Lawrence Berkeley National Laboratory and the University of Maryland are presented to illustrate some of the complexity in beam characteristics that has been uncovered as increased attention has been devoted to developing a detailed understanding of the source region. Also discussed are methods of using the simulations to infer characteristics of the beam distribution that can be difficult to measure directly.
\end{abstract}

Keywords: Beam simulation; Beam sources; Heavy ion fusion; Space-charge-dominated beams

\section{INTRODUCTION}

To focus sufficient power onto an inertially confined target, it is necessary to accelerate and transport a beam that is highly space-charge dominated. Such a beam supports collective warm-plasma collective modes (Haber et al., 2001a; Lund \& Davidson, 1998) that can persist for times comparable to the beam lifetimes in a typical fusion driver design. Details of the initial beam distribution, which affect the excitation of these collective modes, can therefore affect the evolution of the beam for the entirety of the accelerator. Successful use of end-to-end simulation as a design tool, or to explain experimental measurement, therefore depends on the ability to adequately specify the initial mode excitation. The excitation of such modes depends, in turn, on internal correlations in the beam distribution function. Two parallel methods are currently being explored for adequately specifying the initial beam distribution. The first, to be discussed here, is to conduct "first-principles" simulation from the

Address correspondence and reprint requests to: Irving Haber, Institute for Research in Electronics and Applied Physics, University of Maryland, Energy Research Facility, Building 223, College Park, MD 20742-351 I. USA.E-mail: haber@umd.edu emitter surface to predict the distribution as it enters the accelerator. The second method, as discussed by $\mathrm{A}$. Friedman et al. (pers. comm.), explores the reconstruction of the distribution from the measured characteristics downstream from the emitter region.

A significant difficulty, shared by both methods, in adequately specifying an initial distribution is lack of knowledge as just how precise a specification is needed. It is similarly difficult to determine adequate precision required of measurements of the beam distribution at any stage of the accelerator system. And it is usually challenging to obtain sufficiently detailed and accurate measurements at the number of diagnostic locations that would be ideal for a complete understanding of the detailed relationship between simulations and actual measurement.

The work discussed here is therefore of an ongoing nature that is being constantly refined as more measurements become available. Two experimental systems are discussed along with recent examples of progress on understanding the source characteristics of each. These are the High Current Experiment (HCX; Seidl et al., 2002) sited at the Lawrence Berkeley National Laboratory and the University of Maryland Electron Ring (UMER; P.G. O'Shea et al., pers. comm.). 


\section{HIGH CURRENT EXPERIMENT}

To avoid the development of a current spike at the beam head, it is necessary to avoid the overtaking that occurs when particles back from the beam head have energies sufficiently greater than the particles at the head (Lampel \& Tiefenback, 1983). This is achieved in the HCX source by using an electrode that is relatively close to the emitter surface to control the current. A modest voltage applied to this electrode is then used to turn on the current during the flat portion of the main accelerating pulse. In the first generation HCX diode design discussed here, the transverse electric fields from this control electrode were found to be sufficiently nonlinear to cause distortion in the transverse density profile. The most notable manifestation of this is a very sharp rim of increased density at the beam edge. EGUN and WARP simulations were performed to examine the influence of the control electrode on evolution of the beam profile as well as on the total beam current.

Figure 1 is an example of the successful use of simulation to reproduce experimental measurement. The experimental curves were obtained by an array of Faraday cup current probes. The current cross section was measured by rotating the array to obtain a set of curves as a function of angle. These curves were then corrected for the error introduced by the offset of the beam center from the center of the measurement array. Also plotted on the same axes are the curves generated by the EGUN and WARP codes. It should be noted that the simulated current was adjusted to agree within a few percent with the measured value by adjusting the applied voltage in the simulation. This same adjustment was used in both the EGUN and WARP simulations. Note that the WARP curve predicts a sharper peak at the beam rim than both the measured data and the EGUN curve. This is likely a result of reduced resolution in the measurements, which have an approximately $1-\mathrm{mm}$ aperture, and the relatively small number of rays used in the EGUN calculations. It should be noted that Kapton witness plate data somewhat further downstream from the diode exit plane do exhibit a rim whose sharpness is similar to what is observed in the WARP curve, even though it is integrated over the entire pulse and over many shots.

$001221 \mathrm{n}: \mathbf{1}^{\prime \prime}, V-M a r x=450.1 \mathrm{kV} ; V$-extr=53.5kV

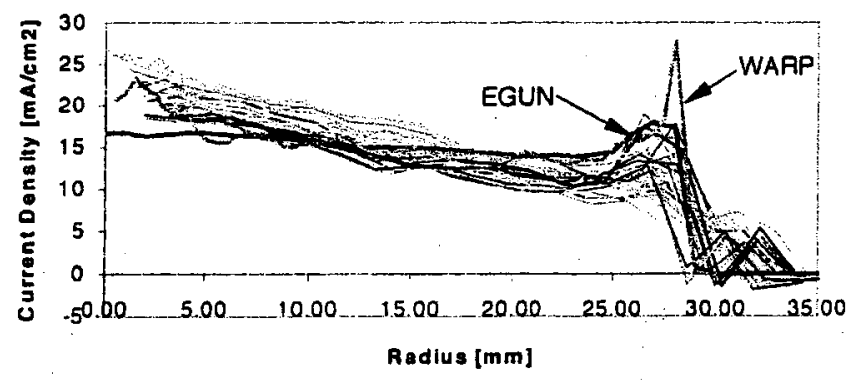

Fig. 1. Measured beam profiles of the beam exiting the source region. Profiles at several angles are compared to EGUN and WARP simulations.
An additional feature of the WARP simulation, which is fully time dependent, is that there are observed oscillations in the density peak at the beam center. The curve shown, however, was integrated over a period of approximately $1 \mu \mathrm{s}$, which matches the temporal resolution of the measured data and therefore is averaged over a large number of the observed oscillation periods.

The degree of agreement seen here was not universally obtained. For example, when the measurement plane was moved $10 \mathrm{~cm}$ downstream, the measured beam radius assuming the same adjustment to the nominal voltage does not increase as much as predicted by the expected space charge spreading. This is thought to result from electrons generated in the field-free region that stream into the beam. Also noteworthy is that a second-generation diode geometry designed to have better control of the beam current, as well as reduced beam nonuniformity, has not exhibited the same level of agreement reported above. This is currently under active investigation.

\section{THE UMER EXPERIMENT}

The gun in the UMER experiment is in many ways different from the HCX structure. Nevertheless many of the issues associated with predicting its behavior are shared with the HCX gun. In the UMER case, an electrode to control the time evolution of the beam pulse was also required. But since UMER is a scaled electron experiment, control of the beam current is effected by the use of a grid close to the cathode. This gridded-gun configuration avoids the gun aberrations associated with a complex control electrode geometry such as is employed in HCX, but it comes at the cost of possible emittance growth from the beam interacting with the grid. Also, since a Pierce gun geometry is generally optimum only for a specific current, variation of the current away from the nominal, as the grid voltage is varied, also changes the current density variation across the beam. This is further complicated because the gun perveance can be varied by mechanically varying the anode-cathode distance.

Simulations of the full gun structure, which include the grid, are quite difficult because of the disparate scales involved. For example, the grid spacing is approximately $0.15 \mathrm{~mm}$, while the grid to anode spacing is approximately $25 \mathrm{~mm}$. Simulations were therefore performed to investigate the sensitivity of the output beam characteristics to variations in the current injected at the plane of the grid, but neglecting the detailed microstructure which results from the interaction of the beam with the cathode grid (Haber et al., $2 n 01 b$ ). Separate simulations of the beam behavior in the cathode-grid region are currently underway to provide a model for the beam distribution function emitted from that region. Measurements of the gun characteristics are also underway to determine an appropriate operating point for UMER operation.

A particular experiment in this connection was the insertion of a five-beamlet mask in a quincunx pattern, similar to 


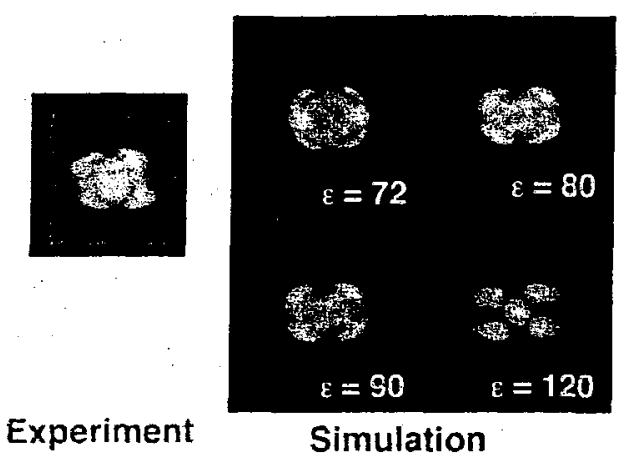

Fig. 2. Comparison of downstream phosphor screen measurement to simulations that assume a range of initial emittances.

the five-dot pattern on dice, as a method of measuring the beam emittance. From previous phosphor screen measurements of the evolution of such a configuration (Haber et al., 1991), it was observed that details of the pattern evolution were a relatively sensitive function of the initial beam emittance. This was used as a method for measuring the beam emittance, as shown for a particular plane in Figure 2. It should be noted that the $4 \times \mathrm{rms}$ beam emittance of $80 \mu \mathrm{m}$, implied by the comparison shown, is more than four times the emittance calculated from the product of the cathode temperature and the beam radius. This is taken as indication that the combination of the disturbance to the beam distribution by the cathode grid and the aberrations in the gun structure can significantly increase the beam emittance.

\section{CONCLUSIONS}

Some preliminary simulations and comparisons to experiment have been presented to illustrate progress in understanding the level of detail necessary to adequately characterize the beam distribution injected into the transport system of experiments currently underway. Because the aim of this work is to develop the capability to predict evolution of a space-charge-dominated beam over long transport distances, this work is ongoing. Refinements will require measurement of beam characteristics over longer propagation distances than have been performed to date. Refinements will also be possible from the incorporation of techniques currently under development to use experimental measurement for the construction of a full distribution function consistent with those measurements.

\section{REFERENCES}

Haber, 1., KehNe, D., Reiser, M. \& Rudd, H. (1991). Experimental, theoretical and numerical investigation of the homogenization of density nonuniformities in the periodic transport of a space-charge dominated beam. Phys. Rev. A 44, 5194.

Haber, I., Friedman, A., Grote, D.P., Lund, S.M., Bernal, S. \& KISHEK, R.A. (2001a). Recent progress in heavy ion fusion simulations. Nucl. Instr. Meth. Phys Res. A 464, 343.

Haber, 1., Bernal, S., Kishek, R.A., O'Shea, P.G., Reiser, M., VALFELLS, A. \& Grote, D.P. (2001b). Computer simulation of the UMER electron gun. Proc. 200J Particle Accelerator Conf. Piscataway, NJ: IEEE.

LAMPEL, M. \& TiefenbaCK, M. (1983). An applied voltage to eliminate current transients in a one-dimensional diode. Appl. Phys. Lett. 43, 57.

LuND, S.M. \& DAvidson, R.C. (1998). Fluid description of intense beam equilibrium and electrostatic stability Properties. Phys. Plasmas 5, 3028.

Seidl P.A., Baca, B., Bieniosek, F., Faltens, A., Lund, S.M., Molvik, A.W., Prost, L.R. \& Waldron, W.L. (2002). The High Current Experiment: First Results. Laser Part. Beams 20. $\mathbf{x x x}-\mathbf{x x}$. 


\title{
Collective Space-Charge Phenomena in the Source Region
}

\author{
I. Haber ${ }^{a}$, S. Bernal ${ }^{a}$, C. M. Celata ${ }^{b}$, A. Friedman ${ }^{b}$, D. P. Grote,
}

\author{
R. A. Kishek ${ }^{a}$, B. Quinn ${ }^{\text {a }}$,P. G. O'Shea ${ }^{a}$, M. Reiser ${ }^{a}$, J.-L. Vay ${ }^{b}$ \\ a Institute for Research in Electronics and Applied Physics, University of Maryland, \\ College Park, MD 20742-3511 \\ ${ }^{\mathrm{b}}$ Heavy Ion Fusion - Virtual National Laboratory, Berkeley, CA 97420
}

\begin{abstract}
For many devices space-charge-dominated behavior, including the excitation of space-charge collective modes, can occur in the source region, even when the downstream characteristics are not space-charge-dominated. Furthermore, these modes can remain undamped for many focusing periods. Traditional studies of the source region in particle beam systems have emphasized the behavior of averaged beam characteristics, such as total current, rms beam size, or emittance, rather than the details of the full beam distribution function that are necessary to predict the excitation of collective modes.

A primary tool for understanding the detailed evolution of a space-chargedominated beam in the source region has been the use of simulation in concert with detailed experimental measurement. However, "first principle" simulations beginning from the emitter surface have often displayed substantial differences from what is measured. This is believed to result from sensitivities in the beam dynamics to small changes in the mechanical characteristics of the gun structure, as well as to similar sensitivities in the numerical methods.

Simulations of the beam in the source region using the particle-in-cell WARP code and comparisons to experimental measurements at the University of Maryland are presented to illustrate the complexity in beam characteristics that can occur in the source region. In addition, direct measurement of the beam characteristics can be limited by lack of access to the source region or by difficulties in obtaining enough data to completely characterize the distribution function. Methods are therefore discussed for using simulation to infer characteristics of the beam distribution from the data that can be obtained.
\end{abstract}

\section{Introduction}

The precision normally associated with beam optics calculations in accelerators has not always been observed in benchmarks against experiments when space charge is important. Furthermore, the influence of space charge in the source region of a machine can be significant even when it is less important as the beam is accelerated. Even when space charge is not an important factor in the behavior of the bulk of an accelerator system, it can therefore still be important to include the influence of space charge in system design. To begin with, the characteristics of the source region determine the initial state of the beam, and characteristics of the initial state such as beam emittance and halo formation [1,2] are often quite important to downstream behavior. Less apparent is 
that there are conditions where space-charge collective modes can be excited in the source region that persist for hundreds of focusing periods.

If one considers the transition from beams of low intensity where space charge can be neglected, as intensity is increased the consequences of space charge forces can often be correctly calculated by assuming a simple model. In this model the space charge contributes an average defocusing force which modifies the beam envelope evolution and modifies the various resonance frequencies. However, as intensity is increased further, a qualitative transition occurs in beam behavior and plasma-like collective modes can become important to describing the beam evolution. Because the excitation of these modes depends on details of the beam distribution function in velocity space as well as configuration space, prediction of the evolution of these modes requires knowledge of the initial phase space characteristics that can be difficult to measure. In addition, variations in the "as-built" transport system characteristics associated with tolerances in construction and operating conditions can also have a significant effect on transport characteristics.

Many of the concepts developed to study nonneutral plasma [1,3] are applicable to the study of a space-charge-dominated beam. A parameter of importance to the beam dynamics is the ratio of beam radius, $r_{b}$, to the Debye length $\lambda_{D}=v_{t h} / \omega_{p}$, where, nonrelativistically, the beam kinetic energy is $1 / 2 \mathrm{mv}_{\mathrm{th}}{ }^{2}$ and the plasma frequency $\omega_{\mathrm{p}}=$ $\left(4 \pi \mathrm{e}^{2} / \mathrm{m}\right), \mathrm{n}$ is the particle density, e the particle charge and $\mathrm{m}$ the particle mass. When this ratio $r_{b} / \lambda_{D}$ significantly exceeds unity the beam is able to support plasma-like oscillations, since such oscillations usually do not occur at wavelengths less than $\lambda_{\mathrm{D}}$.

Description of the beam when it is the space-charge-dominated beam has proved complex. For example, instabilities have been predicted theoretically using the idealized Kapchinskij-Vladimirskij [3] distribution [1,3,5-7], although the applicability of these instabilities to more realistic distributions has usually relied on simulations $[6,8,9]$. More recent work [10] has used a warm-fluid theory to demonstrate that the characteristics of many of the collective space-charge modes do not depend on details of the velocity distribution function. These mode, therefore, occur for more general distributions than the $\mathrm{K}-\mathrm{V}$ distribution originally used in their derivation. However, there still is little theoretical understanding of the mechanism by which these modes are damped and simulations predict that some of the modes can persist for hundreds of focusing periods. It is therefore important to understand the excitation of these modes even for systems that are not space-charge-dominated for the most of their length.

\section{The University of Maryland Electron Ring (UMER) Source}

In view of complex phenomena found to occur in space-charge-dominated beams and the difficulty of understanding how to adequately model these phenomena, the UMER experiment is being constructed to study the fundamental physics of a space charge dominated beam. Since much of this physics depends on details of the six dimensional particle distribution function, it is important to adequately characterize the initial beam distribution. However, measurement of the detailed six dimensional phase space distribution is not usually practical. First principles modeling of the beam source can therefore represent an important technique for inferring details of the beam distribution from the measurements that are possible. Such simulations are also an important technique for characterizing what level of description is required to adequately 
predict the downstream dynamics. Therefore, as the ring is being assembled and diagnostics tested, simulations are being performed to match existing data to extract as many details as possible about the beam characteristics.

Important questions about the sensitivity of the beam characteristics to small deviations from the nominal design can be addressed in some of these preliminary comparisons. These comparisons should therefore be important to understanding the operation of the injector including any small deviations from the nominal design. As will be discussed below, some of the initial findings were not expected and therefore much of what is presented here is part of an ongoing program to refine our understanding of the UMER source operation.

The gun in UMER is Pierce geometry with a $4 \mathrm{~mm}$ radius source, with a gun perveance that can be varied by changing the voltage of the cathode grid and by moving anode plane. For the measurements discussed below the anode to cathode distance is adjusted to approximately $25 \mathrm{~mm}$ so that the current obtained is the nominal $100 \mathrm{~mA}$ beam assumed in the ring design. One difference from standard pierce geometry is an anode grid that transmits approximately $87 \%$ of the beam, and which was employed based on EGUN simulations that predicted a reduction in aberrations by ensuring that the anode plane is an equipotential surface. A more substantial deviation in operation from a standard Pierce diode results from a cathode grid place $0.15 \mathrm{~mm}$ from the planar cathode surface. Since the separation between the $0.025 \mathrm{~mm}$ diameter wires in the rectangular grid pattern is $0.15 \mathrm{~mm}$, i.e. the same as the distance to the cathode, the field pattern in the vicinity of the emitter surface is complex, especially when the space-chargedominated beam is included. The grid is biased negatively by a substantial voltage, usually of order $50 \mathrm{~V}$, to impede current flow until a positive pulse is applied to the grid to draw the pulse of current desired for ring injection. Note that because the grid pulser is at $10 \mathrm{kV}$ relative to ground it is difficult to precisely measure the grid pulse current.

A complete description of the extensive set of diagnostics being developed for measuring the dynamics of UMER is beyond the scope of this discussion. The primary diagnostic discussed here is a phosphor screen that can be moved along the injector. A CCD camera is used to record the time-integrated image on that screen. The UMER gun structure also has a set of masks on a plate that can be rotated to insert different masks allowing variation in the size or shape of the beam emerging from the gun, without breaking vacuum.

\section{Five-Beamlet Emittance Measurements}

A previous study of the evolution, in a long solenoid channel, where an electron beam passed through a mask with a quincunx pattern, corresponding to the five spots on a die, revealed that the evolution of the patterns generated on a phosphor screen was sensitive to the beam emittance.[11] The original pattern was found to reemerge somewhat downstream from the original mask. Simulations of this experiment showed that the plane of the image, as well as the shapes of the intermediate patterns, depended on the emittance of the beamlets. This suggested that the five-beamlet mask could be used to estimate the beam emittance emerging from the UMER electron gun.

Figure 1 shows phosphor screen images obtained on the UMER injector $0.99 \mathrm{~m}$ and $1.04 \mathrm{~m}$ downstream from a five-beamlet mask near the anode plane. These patterns are typical of what is observed when the originally masked beam propagates for some 
distance. The planes shown are chosen because they are at the limit of the straight distance that was available in the injector transport line. The downstream limit was chosen since the dependence of the pattern details on emittance tends to exhibit maximum sensitivity when the beam has propagated to near the plane of where the image is formed. Also shown on the same figure are simulations performed for using the WARP PIC code [12] in single slice mode. In this mode the code solves the twodimensional transverse evolution of the beam using $s$, the distance along the transport line, as the independent variable. In these simulations the initial beam before transiting the mask is assumed uniform across the beamlets and the velocity distribution is assumed to be Gaussian and with constant temperature across the beam. The emittances labeled on the simulation curves shown in Fig. 1 are calculated by multiplying the thermal velocity assumed for the beamlets by the total area the beam would have at the anode plane in the absence of the mask. From a comparison between the simulated and measured pattern it is inferred that the best match occurs when the initial normalized emittance of the total beam is between $16 \mu \mathrm{m}$ to $18 \mu \mathrm{m}$. This is compared to approximately $3.6 \mu \mathrm{m}$ which is the intrinsic emittance of the source calculated by multiplying the source radius by the thermal velocity corresponding to a cathode temperature of $0.1 \mathrm{eV}$. There are several mechanisms that can cause the source emittance to exceed the intrinsic value. However, because masking the beam at the anode plane tends to reduce the emittance growth that results from aberrations in the gun structure, the likely cause of the bulk of the emittance growth is thought to result form the perturbation to the beam distribution by the cathode grid.

\section{Upstream Beam Measurements}

Figure 2 is a set of pictures taken with the phosphor screen positioned approximately $60 \mathrm{~mm}$ from the anode plate. The leftmost column is phosphor screen data for the full beam taken at different settings of the bias voltages. The center column is a set of plots of the light output that is approximately a linear function of the local current density along a diameter of these plots. The right column is a set of phosphor screen pictures at the same location but with a "pepper-pot" mask, so that the light intensity reflects the velocity distribution at the location of each of the small holes in the mask.

As the bias voltage is increased from its minimum value, the amplitude of the rectangular voltage pulse applied to turn on the current is reduced. At the lowest voltage therefore, the maximum pulse is applied to the cathode grid and it is expected that the grid perturbation of the beam velocity distribution would be maximized. As the voltage is increased, the grid potential comes closer to the value it would float at if it were not energized, and therefore the grid perturbation on the particle distribution is expected to be somewhat reduced. One of the surprising features of the pepper pot measurement is the hollowing in velocity distribution most evident at the lowest bias voltages. This is an apparent indication that the grid is strongly perturbing the initial particle distribution by imparting a substantial amount of transverse directed energy to the majority of the beam particles. As the bias voltage is increased so that the grid-to-cathode potential is reduced, this energy is seen to decrease so that at the highest bias voltage, the velocity distribution begins to more closely resemble the transverse Gaussian distribution expected from a thermionic cathode surface. Another feature of note is that at the intermediate bias 
voltages, an intermediate velocity distribution is observed which does not exhibit as much hollowing but shows a squaring of the velocity distribution that reflects the rectangular grid pattern.

Simulation of the entire gun is not straightforward. The geometry becomes threedimensional because the axisymmetry is broken by the rectangular grid pattern, and there is added complexity introduced by the disparity in scales between the $0.15 \mathrm{~mm}$ dimension characteristic of the grid pattern and the $25 \mathrm{~mm}$ distance form the cathode grid to the anode grid. The simulations discussed here have therefore first concentrated on consideration of a model which does not attempt to directly model the complex behavior in the cathode-grid region, but instead starts the simulation by injecting a specified beam distribution injected at the cathode-grid plane. This distribution is assumed to be transversely uniform, so that no attempt has yet been made to include the direct interception of the beam current by the grid wires occupying $31 \%$ of the grid area. This direct interception is spatially correlated with the transverse acceleration of the beam particles and therefore affects the phase of any short wavelength space-charge oscillations launched by the grid. Inability to get direct agreement between the simulated and measured distributions at the plane of the pepper-pot mask is believed to be a consequence of this approximation in the model. Emphasis was instead placed on the spatial characteristics of the full-beam distribution and an exhibition, using the simulations, of how this distribution could be sensitive function of details of the assumed initial velocity distribution. This is contrary to the normal assumption that, because the kinetic energy in a space-charge dominated beam is much smaller than the space-charge potential energy in a nonuniform beam $[14,14]$, the transverse cross section should not be sensitive to details of the velocity distribution.

Figure 3 is a plot of the transverse current distribution at the measurement plane for an injected distribution that is spatially uniform with a Gaussian velocity distribution with a thermal corresponding to the $0.1 \mathrm{eV}$ cathode temperature. This beam is therefore injected at what is known as the inherent, or intrinsic, emittance. The transverse density variation at the measurement plane was found to be insensitive to the injection energy which was nominally fixed at $20 \mathrm{eV}$. The relative unimportance of any transverse variation which would occur in a self-consistent calculation was demonstrated by running a simulation which calculated the gun behavior by assuming space charge dominated emission at the injection plane. When this was compared to a transversely uniform injected beam with the same total current very little difference was observed.

The simulation shown in Fig. 3 was performed by using the WARP code. The r-z module was used to calculate the full time evolution in the region between the cathode grid and the anode grid. After reaching steady state beam was collected at the anode grid, the current was reduced to reflect the approximately $87 \%$ anode grid transparency and then propagated using the two-dimensional single-slice model the remaining $6 \mathrm{~cm}$ to the measurement plane. It is worth noting that the rms emittance measured at the plane of the grid anode is approximately $6 \mu \mathrm{m}$ compared with the injected intrinsic emittance of approximate $3.6 \mu \mathrm{m}$. This presumable reflects the aberrations in the gun structure.

The profile in Fig. 3 is quite different from what is observed on the phosphor screen. Since the grid is known to perturb the transverse velocity distribution, a second simulation, shown in Fig. 4, was performed with an initial thermal velocity five times the intrinsic value. The rms emittance measured from the simulation was approximately 25 
$\mu \mathrm{m}$, which is more than the $16 \mu \mathrm{m}$ to $18 \mu \mathrm{m}$ inferred from the five-beamlet experiment. The central uniform region in this profile does not match the measured the profiles for the $100 \mathrm{~mA}$ beams, but does resemble the profile measured at the $55 \mathrm{~V}$ bias. At this voltage the velocity distribution measured by the pepper pot is a beginning to look Gaussian. Since the beam at this bias voltage only has a current of $81 \mathrm{~mA}$ compared with the approximately $100 \mathrm{~mA}$ for the other bias voltages, it is not clear how to compare the simulations to the data. Since the beam evolution is not a simple function of the current it will necessary to rerun a series of simulations at the lower current to explore the sensitivities of the output distribution to assumed input distribution function.

By increasing the injected thermal velocity by another factor of two, to ten times the intrinsic thermal velocity, the current profile shown in Fig. 5 is obtained which somewhat resembles the measure distribution. However the measured rms emittance of $49 \mu \mathrm{m}$ at the anode plane is now substantially higher than the emittance inferred from the five-beamlet emittance.

Because the potential energy associated with space charge is normally much greater than the kinetic energy in a space charge dominated beam, it is normally assumed that the transverse density distribution will not be sensitive to the form of the velocity distribution. For example the matched rms beam radius in a transport line will usually remain constant over a wide range of emittance if the beam is sufficiently space-charge dominated. However, motivated by the measurements which indicated that the velocity distribution was far from the normally assumed Gaussian shape, it was decided to run simulations with an initially "double-humped" distribution in the $\mathrm{x}$ and $\mathrm{y}$ directions. That is the distributions for $n\left(v_{x}\right)$ and $n\left(v_{y}\right)$ were constructed by displacing four subspecies by $\pm \alpha v_{\text {th }}$ in $x$ and $y$, where both the constant $\alpha$ and $v_{t h}$, the width of the assumed Gaussian distribution, were varied to investigate the consequence to the shape of the output distribution measured at the plane of the phosphor screen. It should be noted that even though the assumed form of the distribution, which would appear as a fuzzy square on a pepper pot measurement, breaks the axisymmetry, this breaking occurs only in velocity space. Full three-dimensional simulations which were run to check any apparent inconsistency between the symmetries yielded essentially results almost identical to the r-z runs.

Figure 5 is a plot of the radial variation in current density for a distribution with $\alpha$ $=4.0$ and $v_{\text {th }}$ twice the intrinsic value. Note that the shape more closely resembles the measured shape than the curve shown $n$ Fig. 3 . This is despite the fact the rms emittance for this distribution measured at the anode plane is only $12.5 \mu \mathrm{m}$, which approximately half of the emittance in Fig. 3. It should be noted that the shape of the profile is a sensitive function of both $\alpha$ and $v_{\text {th. }}$. This would seem to be an indication that the particular shape observed requires sections of the beam that are not traveling in the same radial direction during traversal of the gun. This type of deviation from laminar is not an unusual feature of finite-temperature gun optics.

An interesting feature of the simulations represented by the plot in Fig. 5 is the small dip near the center of the beam which appears to persist over a relatively wide range of parameters, but is not evident in the plot of density along the cut through the beam. Careful examination of the full two-dimensional phosphor image, however, does reveal a dip in density near the center of the beam that is not seen in the plot of experimental density distribution because it is slightly off-center. Note that this dip is 
seen only when a high quality print of the data is generated. It is also possible that the same small misalignment that causes the hole to be off-centered also diminishes the magnitude of the dip relative to the simulations that assume axisymmetry.

\section{Conclusions}

Simulations have been presented which attempt to infer the consequences of the distortion of the injected distribution function by the cathode grid employed in the UMER gun to control the beam current. While the simulations lack some of the relevant physics, particularly the consequences of the configuration space modulation of the beam by the grid and the correlations of this modulation to the velocity space distortions. Many features of the beam characteristics can be recovered by a combination of the simulations and the indirect measurement. Particularly noteworthy is the sensitivity of the radial shape of the beam density variation at the output from the gun. This sensitivity is not normally observed in a space-charge-dominated transport line.

Further simulations are planned to explore the beam behavior in the grid to cathode region by doing high resolution numerical calculations which attempt to examine the behavior of the beam from first principles. Taken together with further measurements in the vicinity of the gun output, it should be possible to infer details of the evolution the full six-dimensional beam distribution in the gun region not normally measured. Since there are many devices whose operation depends strongly on the characteristics of the beam emerging from the source, a more detailed understanding of the evolution of the six-dimensional distribution function should promote designs which result in improved beam characteristics. 
References

[1] Martin Reiser, Theory and Design of Charfed Particel Beams, (John Wiley and Sons, Inc., New York, 1994)

[2] Thomas P. Wangler, "Beam Halo in Mismatched Proton Beams," proc. of this conf.

[3] Ronald C. Davidson, Physics of Nonneutral Plasmas, (Addison-Wesley, Redwood City, CA, 1990).

[4] I. M. Kapchinskij and V. V. Vladimirskij, "Limitations of Proton Beam Current in a Strong Focusing Linear Accelerator with Beam Space Charge," Proc. Int. Conf. on High Energy Accelerators and Instrumentation, ed. by L. Kowarski, p. 274 (CERN, Geneva, 1959).

[5] R. L. Gluckstern, "Oscillation Modes in Two Dimensional Beams," Proc. Of the Linac Conference, Fermilab, 1970, p811.

[6] I. Hofmann, L. J. Laslett, L. Smith and I. Haber, "Stability of the KapchinskijVladimirskij (K-V) Distribution in Long Periodic Transport Systems", Particle Accelerators 13, 145 (1983).

[7] T. F. Wang and L. Smith, ", "Transverse-Longitudinal Coupling in Intense Beams," Particle Accelerators, 12, 247, 1982

[8] I. Haber, "Space Charge Limited Transport and Bunching of Non K-V Beams", IEEE Tran. Nucl. Sci. NS-26, 3090. (1985)

[9] I. Haber, A. Friedman, D. P. Grote, S. M. Lund, and R. A Kishek, "Recent Progress in the Simulation of Heavy Ion Beams,". Phys. Plasmas, Vol. 6, (May, 1999) 2254

[10] Steven M. Lund, Ronald C. Davidson, "Warm Fluid Description of Intense Beam Equilibrium and Electrostatic Stability Properties, Phys. Plasmas, 5, 3028 (Aug. 1998)

[11]I. Haber, D. Kehne, M. Reiser, and H. Rudd, "Experimental, Theoretical and Numerical Investigation of the Homogenization of Density Nonuniformities in the Periodic Transport of a Space-Charge Dominated Beam," Phys. Rev. A15, 44, 5194 (Oct. 15, 1991).

[12]D. P. Grote, A. Friedman, I. Haber, W. Fawley, J. L. Vay"New Developments in WARP: Progress Toward End-to-End Simulation," Nucl. Instr. and Meth, in Phys Res. A, 415, 428 (1998).

[13] Reiser, struck J. Struckmeier, J. Klabunde and M. Reiser, "On the Stability and Emittance Growth of Different Phase-Space Distributions in a Long Magnetic Quadrupole Channel", Particle Accelerators 15, 1344 (1984). 
[14]T. P. Wangler, K. R. Crandall, R. S. Mills, and M. Reiser,"Relation between Field Energy and RMS Emittance in Intense Particle Beams," IEEE Trans. Nuc. Sci. NS$32,2196(1985)$. 
Figure Captions

Fig 1. Phosphor screen data taken 99and $104 \mathrm{~cm}$ from the anode plane when the beam is passed through a mask near the anode plane that creates a pattern of beam current of five beamlets. The initial pattern resembles the five dots on a die. Also shown are a series of simulations that vary the initial temperature to determine what initial temperature range will match the measured pattern. The labels on the simulation curves correspond to the normalized emittance for the full beam with the temperature of the beamlets.

Fig. 2. Phosphor screen data, at a plane $6 \mathrm{~cm}$ from the anode, for different values of the bias voltage on the cathode grid. The left column is a picture of the full beam. The center column is of an intensity scan a cross abeam diameter. The right pattern is the phosphor screen pattern generated by a pepper pot mask near the anode plane and therefore measures the velocity distribution at each of the holes in the pepper pot mask.

Fig. 3. Plot of the beam density vs. radius of a simulated beam at the same $6 \mathrm{~cm}$ plane as the data in Fig. 2. The initial distribution injected at the cathode grid plane is transversely uniform in density and Gaussian in velocity distribution with a spread corresponding to the $0.1 \mathrm{eV}$ intrinsic cathode temperature.

Fig. 4. Beam density vs. radius for a simulation similar to the plot in Fig. 3 but the thermal spread of the initial Gaussian distribution has been increased by a factor of five.

Fig. 5. Beam density vs. radius for a simulation similar to the plot in Fig. 3 but the thermal spread of the initial Gaussian distribution has been increased by another factor of two so that it is ten times the value in Fig. 3.

Fig. 6. Beam density vs. radius for a simulation with an initial distribution which is "double-humped" in the $\mathrm{x}$ and $\mathrm{y}$ directions. The initial beam is constructed as the sum of four subspecies that are uniform in configuration space. However each of the subspecies has a thermal spread twice the intrinsic value, but each is offset by $\pm 4.0 \times v_{t h}$, i.e. four times this thermal spread, in either $x$ or $y$. 
Experiment $\Rightarrow$

Simulation $\Downarrow$

r

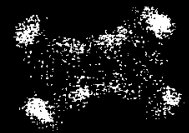

$y$

4

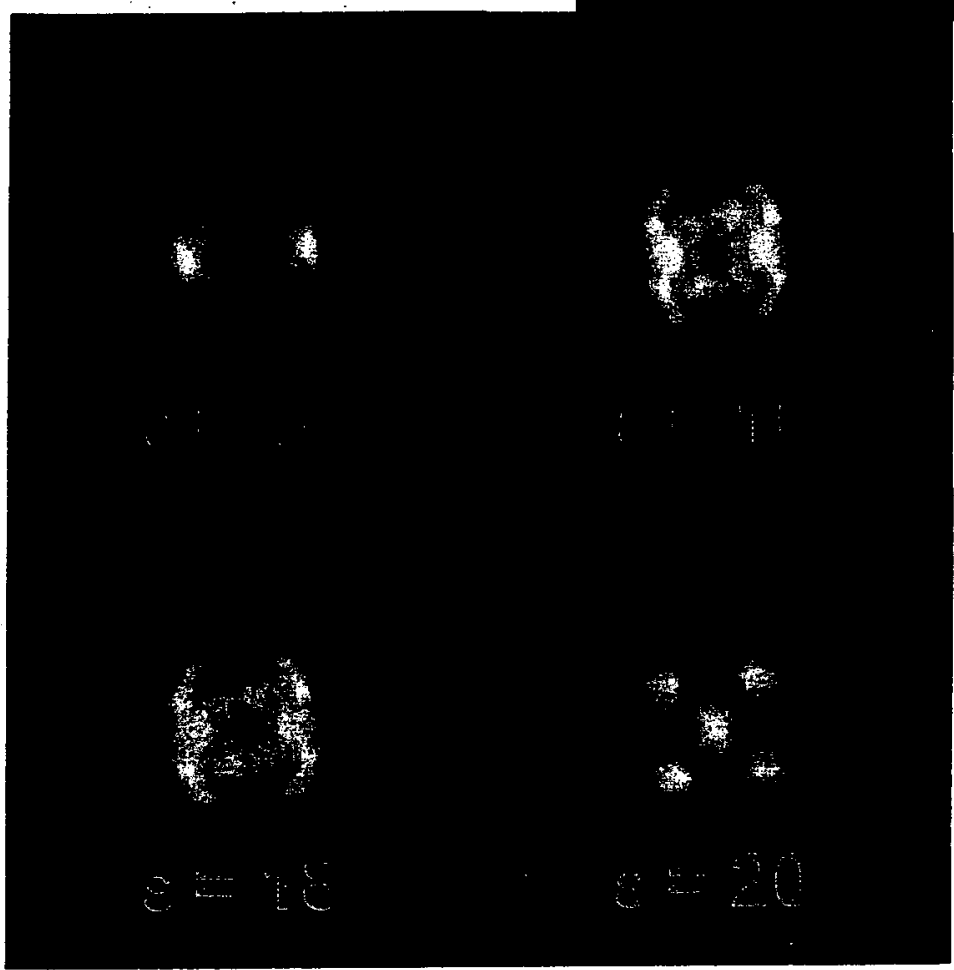

棌

$99 \mathrm{~cm}$

\section{Experiment $\Rightarrow$}

Simulation $\Downarrow$

$104 \mathrm{~cm}$

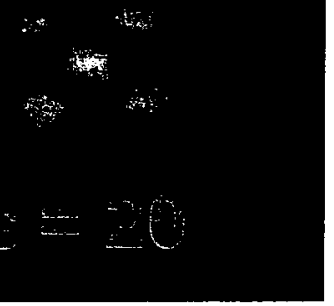

\section{.}

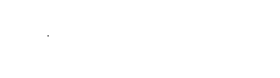


$45 \mathrm{~V}$
$(101 \mathrm{~mA})$

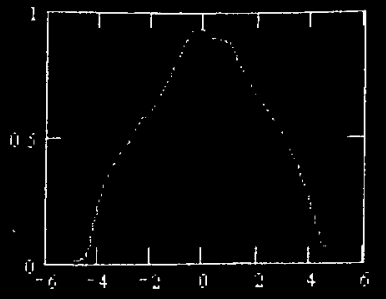

$50 \mathrm{~V}$

(100.8 mA)

$52 \mathrm{~V}$
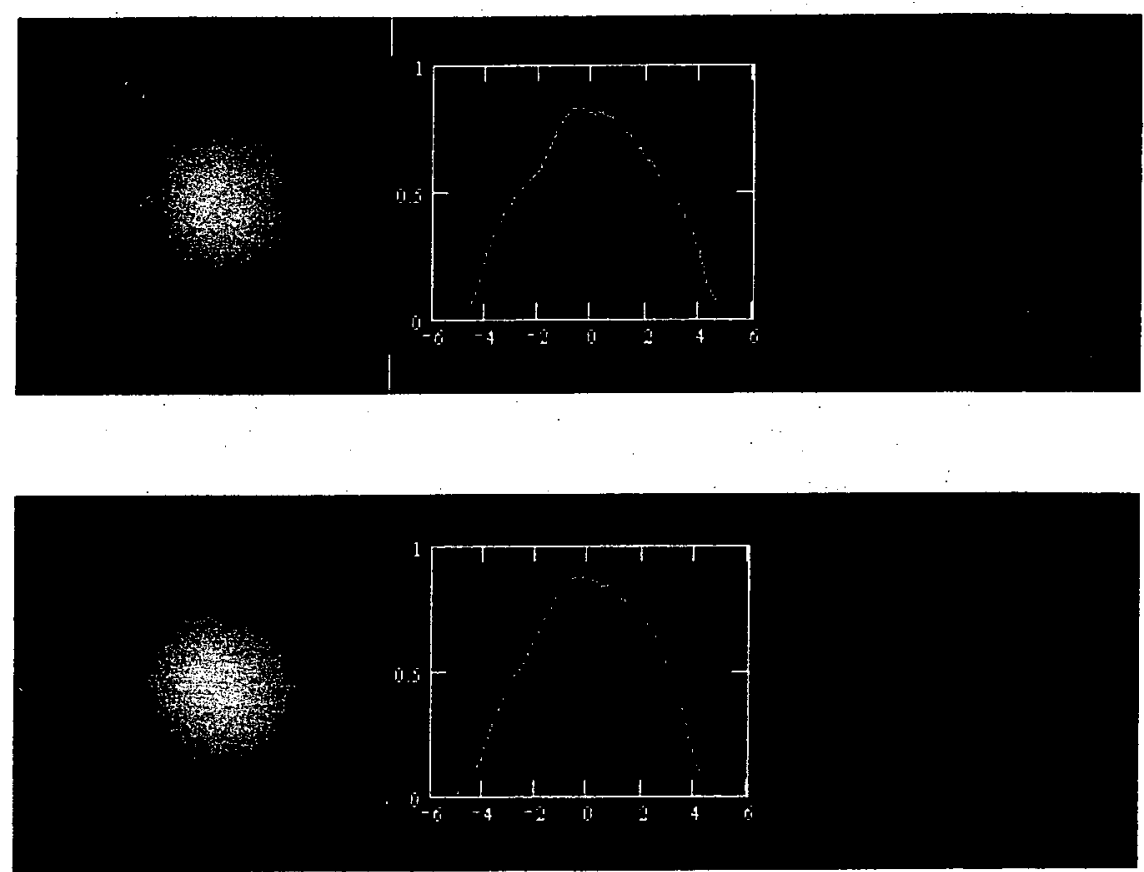

$55 \mathrm{~V}$

(81 mA)

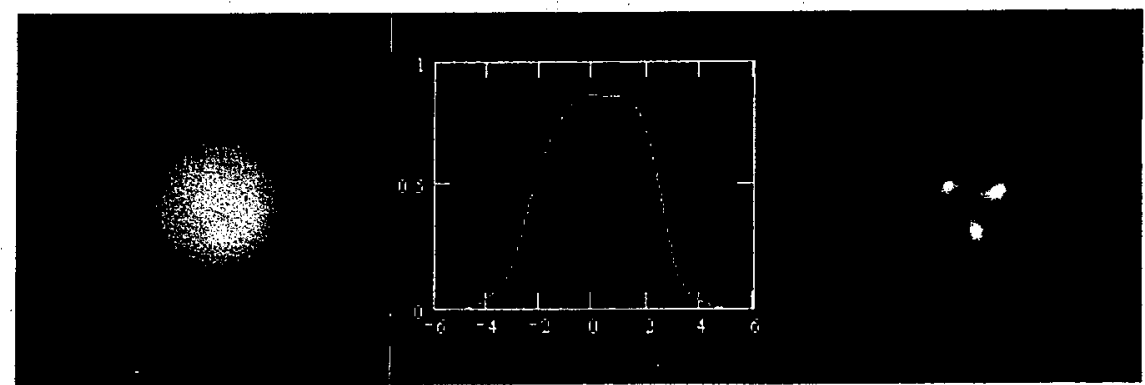




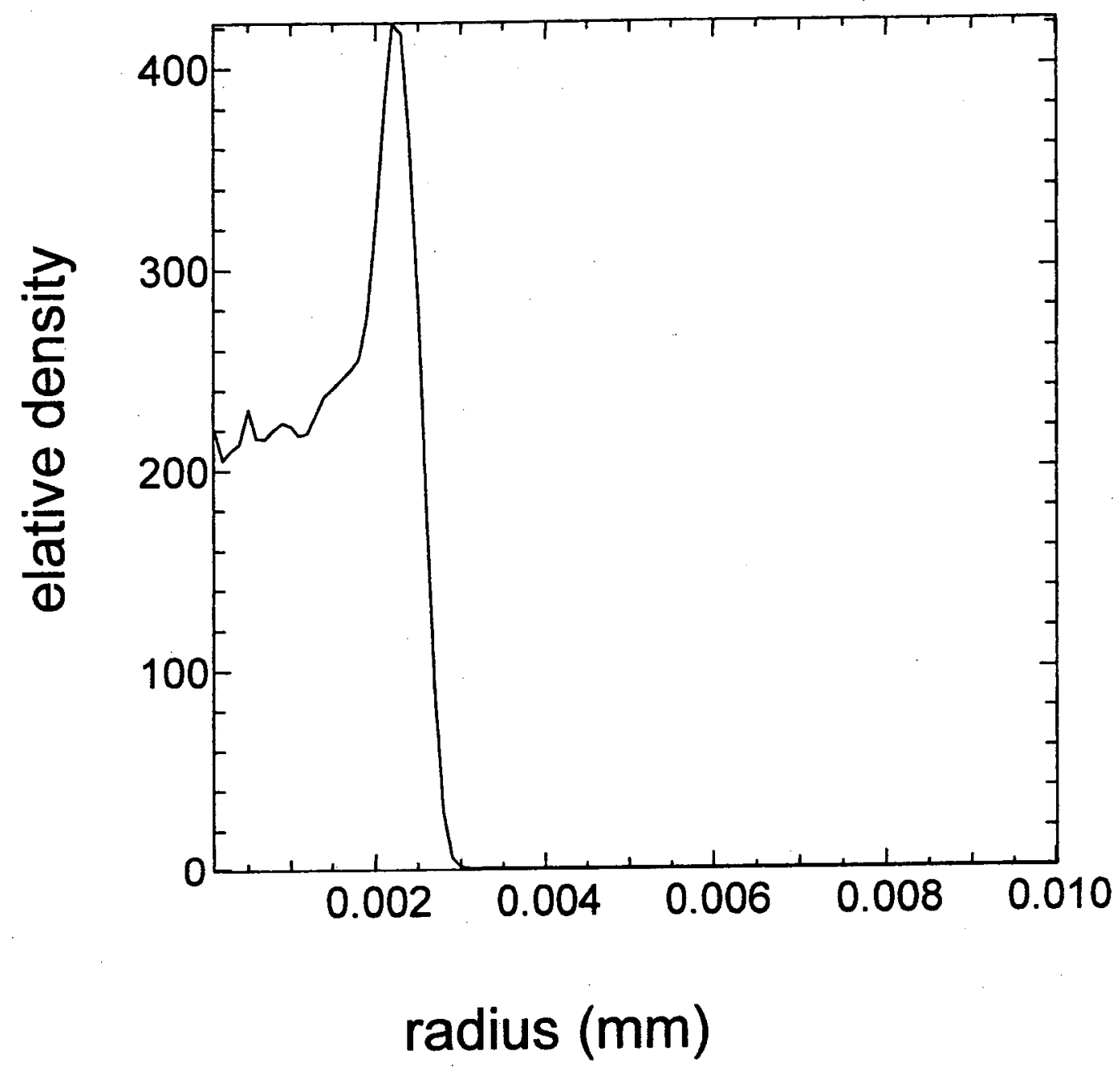




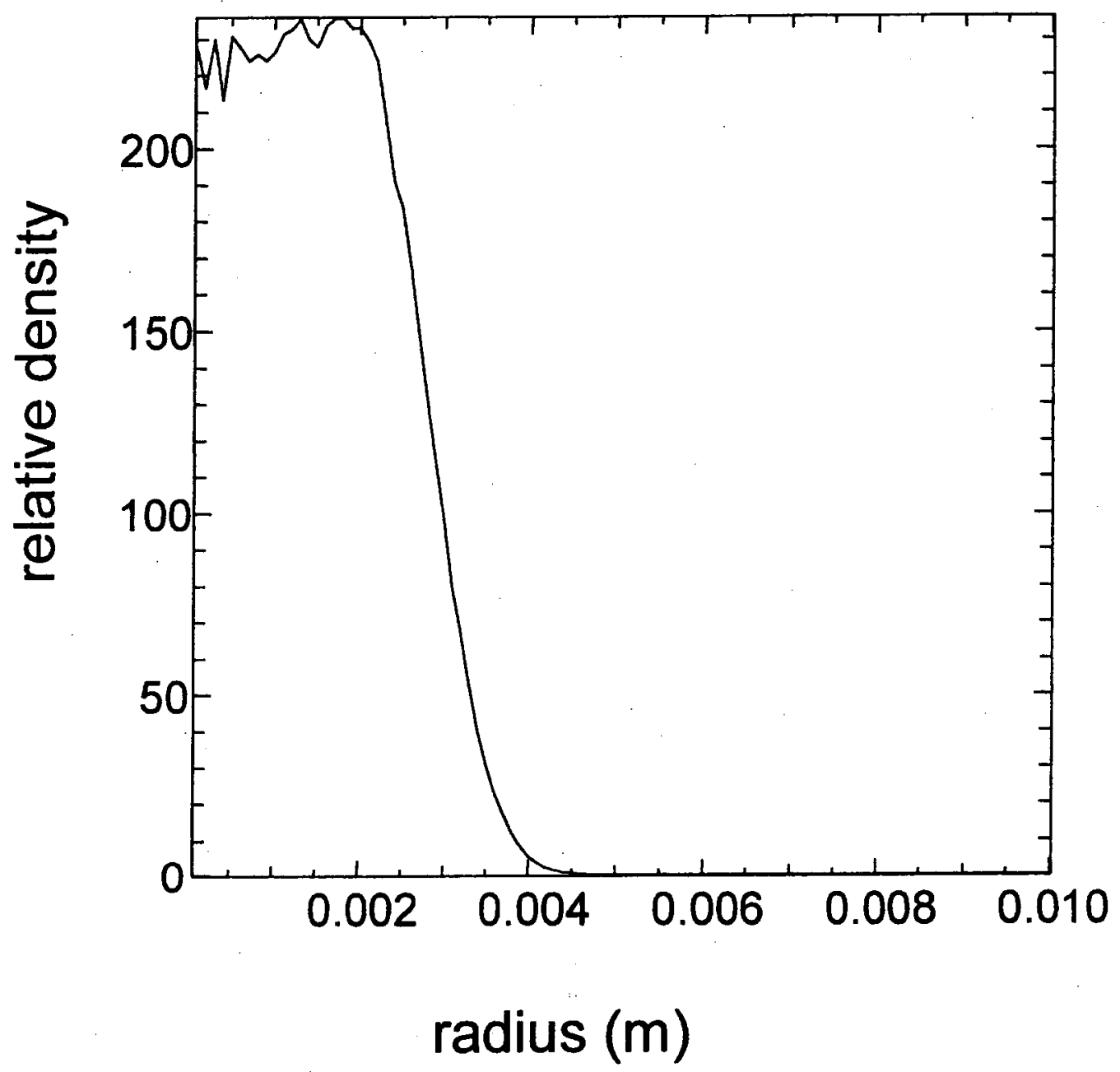




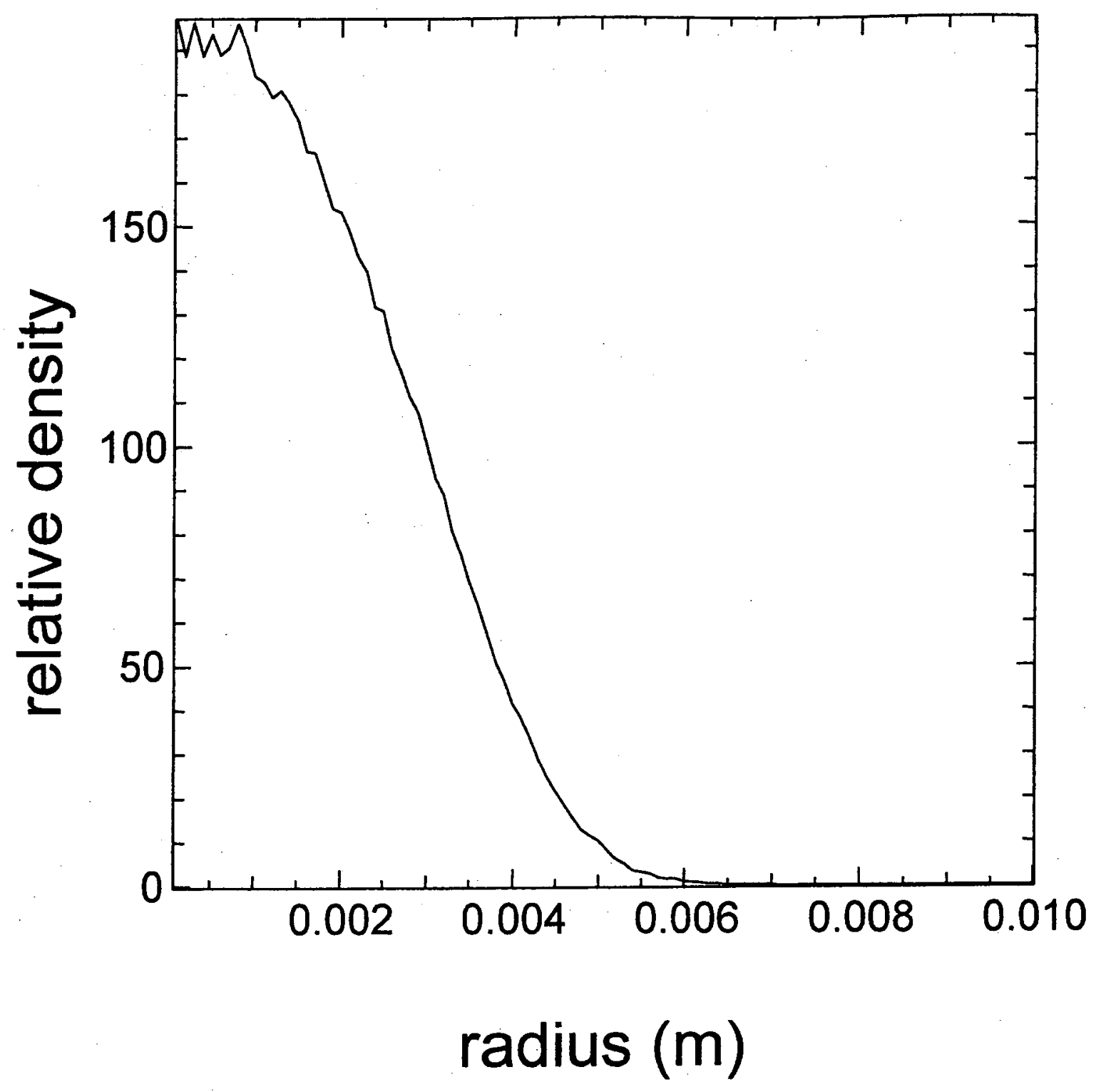




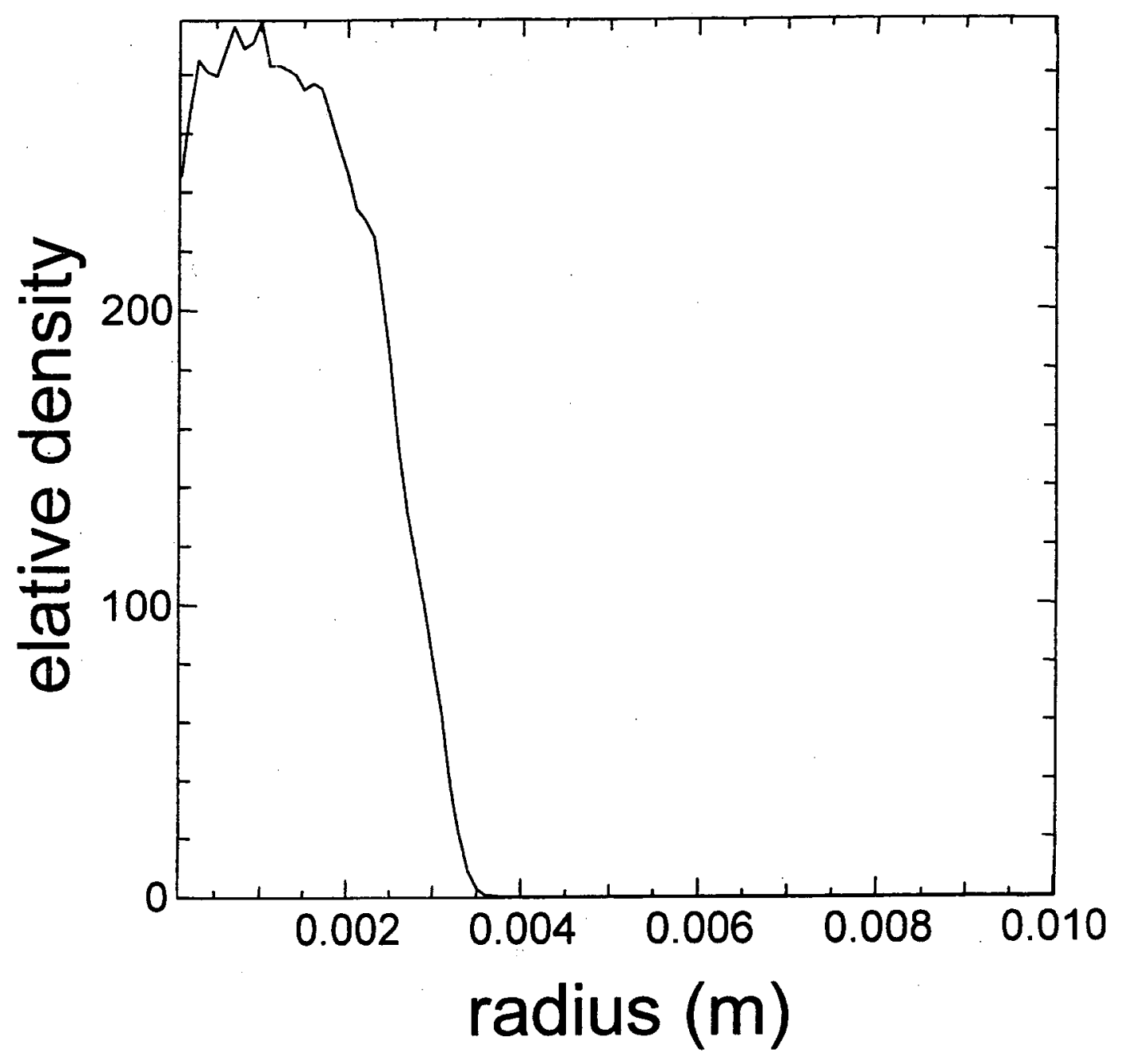




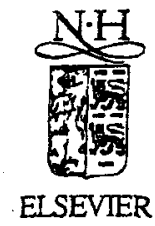

Nuclear Instruments and Methods in Physics Research A 464 (2001) 563-568

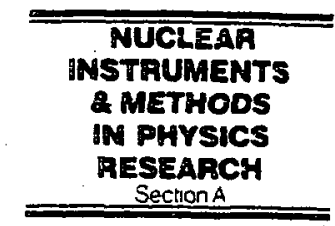

www.elsevier.n//locate/nima

\title{
Progress toward source-to-target simulation ${ }^{\text {th }}$
}

\author{
D.P. Grote ${ }^{a, *}$ A. Friedman ${ }^{\text {a }}$, G.D. Craig ${ }^{a}$, W.M. Sharp ${ }^{a}$, I. Haber ${ }^{b}$ \\ ${ }^{a}$ Lawrence Livermore National Laboratory, Livermore, CA 94551-0808, USA \\ ${ }^{\mathrm{b}}$ Naval Research Laboratory, USA
}

\begin{abstract}
Source-to-target simulation of an accelerator provides a thorough check on the consistency of the design as well as a detailed understanding of the beam behavior. Issues such as envelope mismatch and emittance growth can be examined in a self-consistent manner, including the details of accelerator transitions, long-term transport, and longitudinal compression. The large range in scales, from centimeter-scale transverse beam size and applied field scale-length, to meter-scale beam length, to kilometer-scale accelerator length, poses a significant computational challenge. The everincreasing computational power that is becoming available through massively parallel computers is making such simulation realizable. This paper discusses the progress toward source-to-target simulation using the WARP particle-incell code. Representative examples are shown, including 3-D, long-term transport simulations of Integrated Research Experiment (IRE) scale accelerators. (C) 2001 Elsevier Science B.V. All rights reserved.
\end{abstract}

PACS: $29.27-\mathrm{a} ; 29.27 . \mathrm{Eg} ; 52.65 . \mathrm{Rr}$

Keywords: Accelerator; Fusion; Heavy-ion; Induction; Simulation; Particle-in-cell; Plasma; Beam

\section{Introduction}

A fundamental issue in heavy-ion-driven inertial confinement fusion energy is focusing the ion beam down onto the target. Whether or not this can be achieved depends on nearly all aspects of the system, from the required spot size (the target design), the chamber conditions, the final focus

\footnotetext{
This work was performed under the auspices of the U.S. Department of Energy by University of California Lawrence Livermore National Laboratory under contract No. W-7405Eng-48, and by Naval Research Laboratory, and Lawrence Berkeley National Laboratory under contracts DE-AIO293ER40799, DE-AI02-94ER54232, and DE-AC03-765F00098.

*Corresponding author. Tel.: + 1-510-495-2961; fax: +1510-486-5392.

E-mail address: dpgrote@lbl.gov (D.P. Grote).
}

standoff, and the state of the beam, to name a few. The state of the beam depends on such issues as the design of the accelerator, the error tolerances, the behavior of waves on the beam, the ion source, etc. A complete understanding of the state of the beam at the target requires that the issues be examined in an integrated manner. Computer simulation is an ideal tool for this integrated examination. In this paper is discussed some of the progress toward complete source-to-target simulation of the beam.

The goal of the source-to-target simulation is to provide a fully consistent, fully integrated model of the beam, tracking the same particles from creation in the ion source, through acceleration and transport, through final compression and focus, and to the target. Tracking the same 
particles through the system removes any approximations that would be made by re-initializing the beam at the start of each section using low-order moments from the end of the previous section. The full detail of the dynamics is carried over from section to section.

The principal code being developed for the source-to-target simulation is the WARP [1] code. WARP combines the particle-in-cell (PIC) method from plasma physics with a detailed description of the accelerator lattice. The PIC method captures the beam dynamics, including the self-field, in a self-consistent manner. The model can either be three-dimensional (WARP3d), or two-dimensional, axisymmetric (WARPrz) or transverse-slice (WARPxy). The description of the lattice includes accelerator elements having idealized axially uniform, hard-edged fields, as well as generalized elements where the applied field is specified either via axially varying multipole components or via data on a three-dimensional mesh. Electric elements can also be included self-consistently by including the geometry of the element as a boundary condition of the solution of Poisson's equation for the self-field. Additionally, there is an envelope solver which is primarily used to calculate the matched beam parameters in order to load the particles for the PIC models, when not injecting from the source.

Much work has been carried out in simulating various aspects of an accelerator. In these proceedings are papers describing simulation of the ion source and injector system [2,3], final focus [4], and propagation in the fusion chamber $[5,6]$. In this paper is discussed simulations of the acceleration section, after injection and before the final compression. This section is likely the longest and the most complex.

An additional aspect of the source-to-target work is general code development. This work is described elsewhere. Two major areas are development of improved methods of simulation, such as improved algorithms for particle codes and Vlasov methods $[7,8]$, and development of models for the high-energy end of the accelerator, such as Darwin field models for magnetostatic and inductive fields [8], and multiple beam models [9].

\section{Transverse slice simulations}

Considerable understanding of the beam behavior in the accelerating section can be obtained using the transverse slice model. The model includes the two transverse position coordinates and all three velocity coordinates. A major use of the slice model is characterization of the design tolerances and allowable errors.

For a place to begin the simulations, a preliminary design of an IRE [10] scale accelerator was drafted. The design is not necessarily optimized and is only representative. Some of the parameters and scaling relations are given in Table 1. the design consists of the three sections. In the first, electric transverse focusing is used and the beam length (in meters) is held constant. In the second section, which begins the magnetic transverse focusing, the beam is compressed longitudinally. In the final section, the beam length is held constant and the accelerating gradient is at its maximum value. Using the continuous scaling relations and the transition points, a discrete lattice is laid out, ignoring the discontinuities in the velocity tilt. For the slice simulations, no accelerating wave-forms need to be generated. Considerable work has been carried out characterizing a number of machine and beam errors in the IRE design [11].

One issue is the alignment tolerance of the focusing quadrupoles. Any transverse offsets will kick the beam off-axis, possibly (in combination with non-linear applied fields and image forces) causing emittance growth and in the worst case causing some of the beam to be lost by striking the surrounding walls. In earlier work [11], the magnitudes of random quadrupole offsets were set so that, over the course of the simulation, little of the beam was lost to the walls. While it was known that the magnitudes of the errors were smaller than what could currently be achieved in experiment, the centroid displacements of the beam were representative of what would be expected in experiments which include steering.

Simulations using experimentally achievable quadrupole displacement errors along with beam steering have now been carried out. The offset errors in the electric quadrupoles were taken to 
Table 1

The variation of lattice and beam parameters as functions of the beam mid-pulse energy, $v$. There are three sections: in the first electric focusing is used; in the second, magnetic focusing is used and the beam is compressed; and in the third, the beam is not compressed and the accelerating gradient is held constant at its maximum value. The numbers shown are for a specific model design and do not represent an optimal for final design

\begin{tabular}{|c|c|c|c|c|c|c|c|}
\hline $\begin{array}{l}\text { Total energy }=30 \mathrm{~kJ} \\
32 \text { beams of } \mathrm{K}^{+} \text {ions } \\
A=38.96 \mathrm{amu} \sigma_{0}=54^{\circ} ; \\
\sigma i \sigma_{0} \sim 0.1 \mathrm{a}_{\text {mean }}=1.8 \mathrm{~cm}\end{array}$ & $\begin{array}{l}\text { Energy } \\
(\mathrm{MeV})\end{array}$ & $\begin{array}{l}\text { Current } \\
\text { (A) }\end{array}$ & $\begin{array}{l}\text { Pulse duration } \\
(\mu s)\end{array}$ & $\begin{array}{l}\text { Bunch } \\
\text { length } \\
\text { (m) }\end{array}$ & $\begin{array}{l}\text { HIP } \\
\text { length } \\
\text { (m) }\end{array}$ & Occupancy & $\begin{array}{l}\text { Accelerating } \\
\text { gradient } \\
\text { (MV/m) }\end{array}$ \\
\hline Initial . & 1.6 & 0.77 & 6.7 & 18.8 & 0.20 & 0.65 & 0.055 \\
\hline Electric focusing & $v$ & $v^{1 / 2}$ & $v^{-1 / 2}$ & $v^{0}$ & $v^{1 / 2}$ & $v^{0}$ & $v$ \\
\hline E-M transition & 9.4 & 1.9 & 2.8 & 18.8 & 0.50 & 0.33 & 0.32 \\
\hline Compression & $v$ & $v$ & $v^{-1}$ & $v^{-1 / 2}$ & $v^{1 / 4}$ & $v^{0}$ & $v$ \\
\hline Constant accel & $\begin{array}{l}30 . \\
v\end{array}$ & $\begin{array}{l}5.9 \\
v^{1 / 2}\end{array}$ & $\begin{array}{l}0.87 \\
v^{-1 / 2}\end{array}$ & $\begin{array}{l}10.6 \\
v^{0}\end{array}$ & $\begin{array}{l}0.67 \\
v^{1 / 2}\end{array}$ & $\begin{array}{l}0.33 \\
0^{-1 / 2}\end{array}$ & $\begin{array}{l}1.0 \\
0^{0}\end{array}$ \\
\hline Final & 200. & 15. & 0.34 & 10.6 & 1.7 & 0.13 & 1.0 \\
\hline
\end{tabular}

have an RMS of $100 \mu \mathrm{m}$, based on estimated machining limits of the quadrupole structure. The errors in the magnetic quadrupoles are larger, $250 \mu \mathrm{m}$, obtained from estimates of the errors in magnet designs. The error distribution is Gaussian.

The steering used in the simulations is idealized. At the steering station, the centroid position of the beam is subtracted out and a small random error component added back in. To add this error component, which accounts for the steering errors (both in sensing of the beam location and in the fields correcting it), the beam centroid is placed randomly within a circle of radius $200 \mu \mathrm{m}$ about the axis. Any transverse velocity of the centroid is completely removed.

A series of simulations was carried out with different frequencies of steering, ranging from the best-case every half-lattice period (HLP) up to once every $40 \mathrm{HLPs}$. The results are summarized in Table 2. For each of the steering frequencies, six runs were done with differing sets of random errors. The pipe radius is $3.48 \mathrm{~cm}$. The numerical parameters are : $64 \times 64$ field grid, 40,000 simulation particles, 60 steps per half-lattice period. The quadrupole fields include fringes with the pseudooctopole term and the lowest order longitudinal component.

In Fig. 1 are shown some of the results. In all of the cases the emittance growth is within acceptable limits. These simulations provide guidelines for
Table 2

Beam centroid RMS offsets and maxima for transverse slice simulations with realistic quadrupole offsets and ideal steering. The quadrupole offsets are $100 \mu \mathrm{m}$ for the electric quadrupoles and $250 \mu \mathrm{m}$ for the magnetic. The steering places the beam within $200 \mu \mathrm{m}$ of the axis and removes the centroid velocity

\begin{tabular}{lll}
\hline $\begin{array}{l}\text { Steering } \\
\text { frequency (HLPs) }\end{array}$ & $\begin{array}{l}\text { RMS offset } \\
(\mathrm{mm})\end{array}$ & $\begin{array}{l}\text { Max offset } \\
(\mathrm{mm})\end{array}$ \\
\hline 1 & 0.11 & 0.62 \\
5 & 0.3 & 3.7 \\
10 & 0.8 & 4.6 \\
20 & 1.2 & 6.4 \\
40 & 1.5 & 8.0 \\
\hline
\end{tabular}

how steering will be done in a large scale accelerator. They indicate that the beam can be steered back onto the axis fairly infrequently without causing significant degradation. Note that the RMS measure of beam offset is the more important, since the maximum can be anomalously large in the unlikely case that the errors resonate with the betatron motion of the centroid. More simulations are required for a thorough examination of the offsets in combination with other errors and non-linear fields.

\section{Three-dimensional simulations}

There are a good number of issues which cannot be fully examined with the transverse slice model 

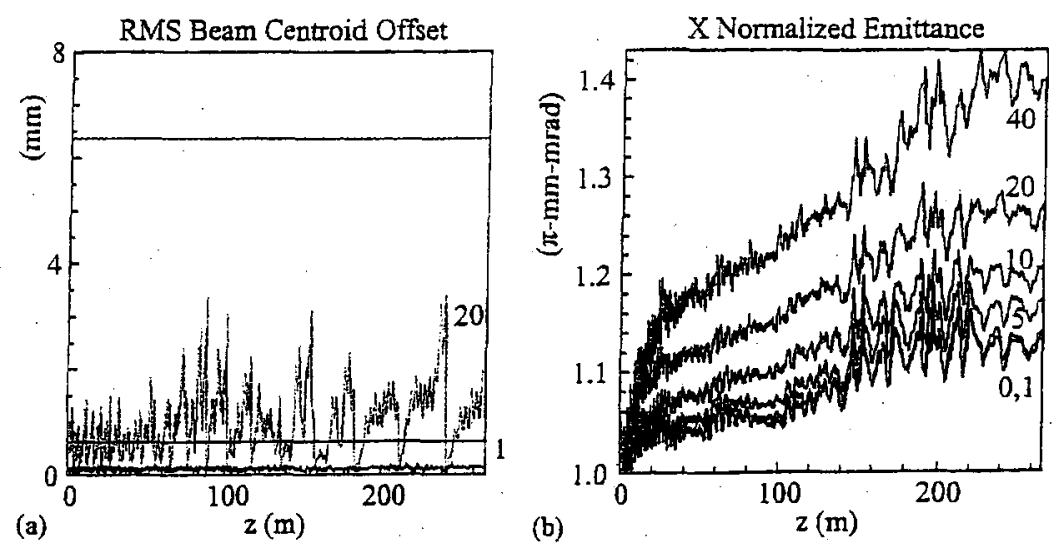

Fig. 1. Results from transverse slice simulations with quadrupole offsets and ideal steering: (a) The RMs beam centroid offset. The black is the ideal case of steering every HLP. The gray is steering every twenty HLPs. The two jagged curves show, for the two cases, the RMS over six simulations for each $z$ location. The horizontal lines show the maximal value over all of the simulations; (b) The $x$ normalized RMS edge emittance for the cases with no errors, and with errors and steering every $1,5,10,20$, and 40 HLPs.

and so require a three-dimensional model. For example, the injection of the beam including transients and the initial "catching" by shaped accelerating waveforms, preventing head and tail expansion; head-to-tail variations in the mismatch and centroid offsets; and errors in the accelerating and ear waveforms.

For a three-dimensional simulation, a complete lattice design is required, including the accelerating waveforms. For the simple case of "load and fire", the voltage gradient on all of the gaps is the same at any time-all of the waveforms can be generated directly from the continuous scaling rules. With "load and fire", the beam drifts out of the injector until it is completely in the accelerator and then all of the gaps are switched on to accelerate the beam as a whole - the beam maintains a constant length. Fig. 2 shows example waveforms in the first section of a model IRE design.

Using the generated waveforms and the associated lattice, three-dimensional simulations were carried out. The long and thin beams require significant computation resources. The numerical parameters used are: grid dimensions $32 \times 32 \times 2048$ for one transverse quadrant, $2,000,000$ particles, and 10,000 time steps. The clock time for the run on 128 processors of

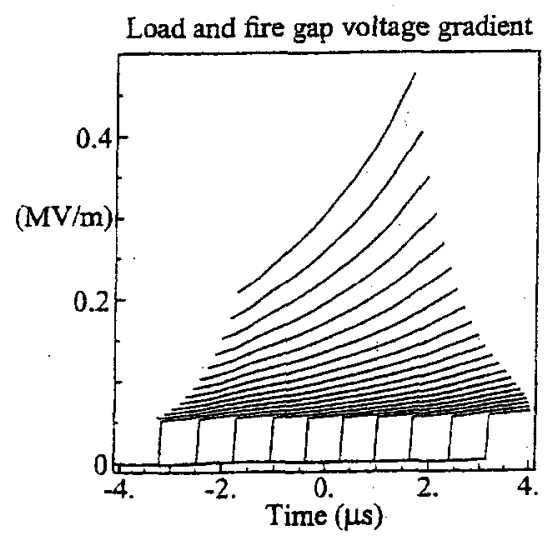

Fig. 2. The gap voltage gradient for every tenth gap using the load and fire procedure, plotted reiative to the time of arrival of the center of the beam at the gap.

the NERSC ${ }^{1}$ T3E-900 was $1.1 \mathrm{~h}$. This simulation was only over the first section of the IRE design, with ideal electric quadrupoles (with no offsets). The beam travels just over $43 \mathrm{~m}$, or 168 HLPs.

As an aside, it is important to note that good parallel efficiency has been obtained for up to

\footnotetext{
${ }^{1}$ National Energy Research Scientific Computing Centre, at Lawrence Berkeley Laboratory, Berkeley, California.
} 
several hundred processors. With more processors, the efficiency begins to drop because of the global nature of the required Poisson solve for the beam self-fields. Improved techniques are being examined. Because of their large scale, efficient use of state-of-art parallel computers is essential for the source-to-target simulations to be possible.

The simulation shows a fairly quiescent beam. Essentially no increase is seen in the transverse normalized emittance (it varies by a few percent). The level of mismatch in the head and tail is small, roughly $5 \%$. The difference in the transverse size in the head and tail is consistent with the velocity tilt, $30 \%$. The global (over the full beam) RMS axial velocity spread $(\Delta \beta / \beta)$ is initially $0.3 \%$ and changes little. The overall spread is due to a combination of three effects: a thermal velocity spread which is initialized to just under $0.3 \%$ (and equal to the transverse thermal velocity spread), and decreases inversely proportional to the increasing velocity; the variation of the axial velocity in the accelerating gaps which is initially $0.35 \%$ and increases with the velocity; and the variation of the head and tail velocities due to imperfect axially confining ear fields which initially is zero and increases to of order $1 \%$. With perfect ears, the estimated velocity spread would be just over $0.2 \%$. It is a coincidence that the three combined in this simulation to give a nearly constant spread. Over the course of the simulation, the local absolute velocity spread $(\Delta \beta)$ changes little.

Further work will begin including element errors, use realistic steering algorithms, examine other accelerating schemes (in addition to "load and fire"), and will include more of the accelerator past the electric focusing section. One alternate scheme to begin the acceleration of the bean can be called "fire and load". Here, the gaps are switched on as the beam enters the accelerator. The first several gaps are tailored to have a rapidly rising waveform to give the beam its initial velocity tilt, i.e. the tail of the beam is accelerated more initially so it keeps up with the head which has gone through more gaps. In Fig. 3 is shown a sample set of waveforms for "fire and load": After the whole beam is in the accelerator, the waveforms of both schemes are similar. The "fire and load" scheme allows shortening of the accelerator length of

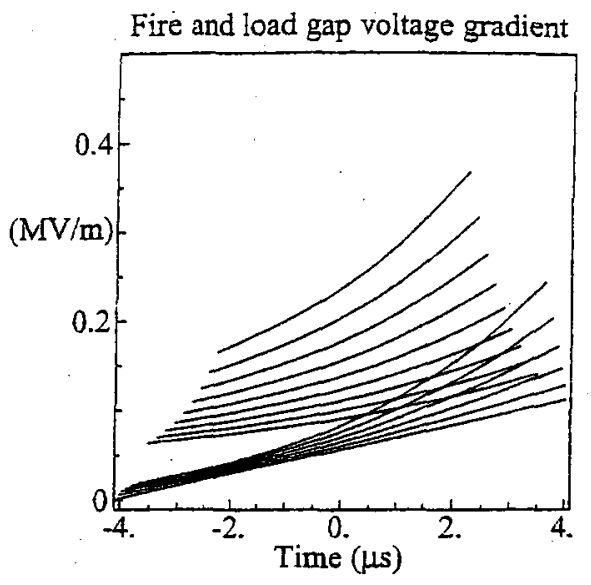

Fig. 3. The gap voltage gradient for every tenth gap using the fire and load procedure, plotted relative to the time of arrival of the center of the beam at the gap. The first six of the curves shown were taiiored to gradually increase the axial velocity tilt.

order the initial beam length and removes constraints on the rise time of the gap voltages, at the expense of more complicated waveforms and a small but unavoidable longitudinal expansion of the beam.

\section{Conclusions}

A thorough validation of the beam dynamics in an IRE or driver accelerator requires integrated source-to-target simulation, tracking the same particles from birth to death. Significant simulations have been carried out of all parts of a largescale accelerator, including injection, acceleration, and final compression and focus. Much progress has been made toward full simulation of the main accelerator section, which is likely the most complex section. Both two- and three-dimensional simulations are being carried out. The next step will be connecting the simulations of the different sections.

\section{References}

[1] D.P. Grote, A. Friedman, I. Haber, S. Yu, Fusion Eng. Des. 32-33 (1996) 193. 
[2] E. Henestroza, D.N. Beck, A. Faltens, D.P. Grote, I.W. Kwan, Multiple-beam ion guns for heavy-ion fusion, Nucl. Instr. and Meth. A 464 (2001), these proceedings.

[3] L. Ahle, D.P. Grote, E. Halaxa, The adiabatic matching section solution for the source injector, Nucl. Instr. and Meth. A 464 (2001) 599, these proceedings.

[4] M.J.L. de Hoon et al., Simulations of the LBNL scaled final focus experiment, Nucl. Instr. and Meth. A 464 (2001) 278 , these proceedings.

[5] W.M. Sharp, M.S. Armel, D.A. Callahan-Miller, A.B. Langdon, J.-L. Vay, Improved modeling of chamber transport for heavy-ion fusion, Nucl. Instr. and Meth. A 464 (2001) 284, these proceedings.

[6] D.V. Rose, A. Friedman, B.V. Oliver, W.M. Sharp, D.R. Welch, Ballistic neutralized chamber transport of intense heavy-ion beams, Nucl. Instr. and Meth. A 464 (2001) 299, these proceedings.
[7] E. Sonnendrucker et al., Simulation of heavy-ion beams with a semi-lagrangian Vlasov solver, Nucl. Instr. and Meth. A 464 (2001) 470, these proceedings.

[8] E. Sonnendrucker, Progress towards simulating heavy-ion beams for inertial fusion energy based on (1) a Darwin model field solver, and (2) a semi-lagrangian Vlasov solver, Proceedings of the 1999 Particle Accelerator Conference, p. 2758.

[9] A. Friedman et al., Beam simulation for IRE and driver-status and strategy, Nucl. Instr. and Meth. A 464 (2001) 653, these proceedings.

[10] J.J. Barnard et al., Planning for an integrated research experiment, Nucl. Instr. and Meth. A 464 (2001) 621 , these proceedings.

[11] A. Friedman et al., Beam dynamics studies for heavy-ion fusion drivers, Proceedings of the 1999 Particle Accelerator Conference, p. 1830. 


\title{
PIC SIMULATIONS OF BEAM DYNAMICS EXPERIMENTS PLANNED FOR THE HIGH CURRENT EXPERIMENT'
}

\author{
C.M. Celata, Lawrence Berkeley National Laboratory, Berkeley, CA, U.S.A. \\ I. Haber, Naval Research Laboratory, Washington,D.C., U.S.A. \\ D.P. Grote, Lawrence Livermore National Laboratory, Livermore, CA, U.S.A.
}

\begin{abstract}
This paper reports progress on simulation of the High Current Experiment ( $\mathrm{HCX})$, a heavy ion accelerator at LBNL built to explore transport of intense beams at the scale of a heavy ion fusion power plant driver. The 2D particle-in-cell (PIC) code WARPxy was used to the explore effect of image forces, distribution function, and focusing nonlinearities on the dynamic aperture of the electrostatic focusing system for the HCX, as well as the effect on dynamics of misalignments and quadrupole rotations. Simulations using a semigaussian initial distribution are compared to results for a more realistic distribution function obtained by initializing a spacecharge-dominated beam at the emitter and propagating it through the aberrations of the injector and matching section.
\end{abstract}

\section{INTRODUCTION}

Phase 1 of the High Current Experiment (HCX) is presently being built by the Heavy Ion Fusion Virtual National Laboratory at Lawrence Berkeley National Laboratory. Phase 1 is a coasting-beam experiment which transports a beam of $\mathrm{K}^{+}$through 40 electrostatic quadrupoles, followed by a four magnetic quadrupoles. Several important experiments are planned for the HCX, including measurements to determine the dynamic aperture of the electrostatic focusing system. The

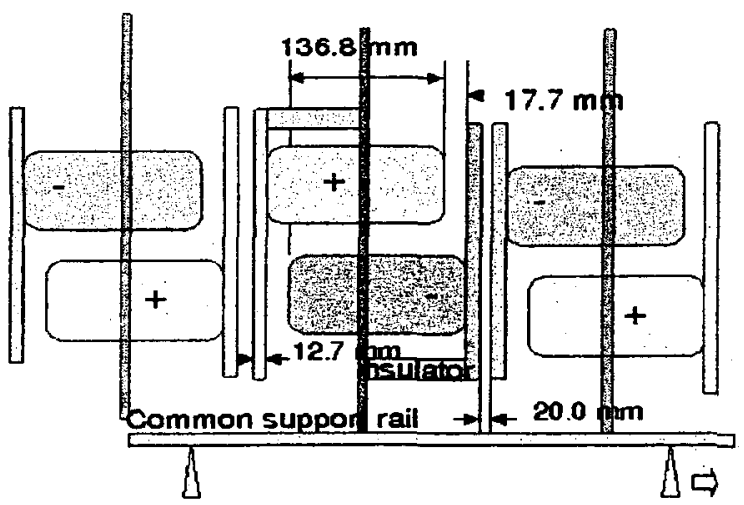

dynamic aperture has great significance for the cost of a heavy ion

Figure 1. HCX Electrostatic Quadrupole FODO Lattice

\footnotetext{
- This work supported by the Office of Energy Research, U.S.

Department of Energy, under contract number DE-AC03-76SF00098.
}

fusion driver, an induction linac which will accelerate many $(\sim 100)$ individually-focused beams in parallel to a target. The front end of this machine is expected to use electrostatic focusing. The cost of the induction cores encircling the focusing arrays is a major cost, and the diameter of these cores depends on the beam aperture needed. For the intense beams of interest, space charge is a major potential source of nonlinearity, and image forces are expected to be important to limitations on the aperture. Simulations of the dynamic aperture experiment using the 2D (transverse) version of the PIC code WARP [1] will be the main focus of this paper. We will also discuss simulations of misalignments of the beam, and quadrupole rotation errors. The HCX will have a rotatable quadrupole, which can be used to measure the effects of quadrupole rotation errors. Steering will also be possible, and can be used both to correct the alignment of the beam coming from the injector, and to intentionally introduce misalignments, in order to study their effects.

\section{SIMULATION PARAMETERS}

The HCX beam is presently envisioned to be a $700 \mathrm{~mA}$, $\mathrm{K}^{*}$ beam at $1.7 \mathrm{MeV}$, with the ratio of depressed to undepressed phase advance $\sim 0.1$. A short length of the FODO electrostatic lattice [2] is shown in Fig. 1. The radial distance from the center of the current channel to the quadrupole electrode surface is $2.30 \mathrm{~cm}$, and the radius of the electrodes is set to $8 / 7$ of this value in order to minimize the dodecapole nonlinearity of the focusing field. The effective length of the quadrupoles is $15.4 \mathrm{~cm}$. For the simulations, the fields of the quadrupoles were first calculated using the 3D version of the WARP code, including the conducting electrodes and quadrupole end plates. At the $\mathrm{z}$ location of each timestep of the 2D simulation, the fields were decomposed into a set of multipole moments. The moments (up to $\cos (10 \theta)$ ) were then used to construct the focusing forces on the particles for the 2D simulation. Thus the 2D simulations included fringe fields of the electrodes, nonlinearities of the field within the quadrupole, and image forces, which were calculated using the capacitive matrix technique.

The irijector which will be used for the HCX has been in operation for some years and the beam has been heavily diagnosed, and simulated in 3D [3,4]. The main features of the beam, including a noticeable rim due to spherical aberration in the diode, are visible in the 
simulation. The beam passes from the diode to an electrostatically focused section of the injector, where the azimuthal symmetry of the diode is broken. In this section the rim breaks into separate peaks in the density distribution, which cause an ongoing density oscillation of particles into center of the beam and then out to the peaks. The nonuniform density of the beam can be decomposed into transverse collective modes of oscillation inherent in space-charge-dominated beams. Though the higher order modes will phase mix and interact, washing out quickly, the oscillation of the lowest order modes persists, giving the beam time-dependent peaks which are seen in the simulations.

The injector is followed by a matching section, which immediately precedes the HCX alternating gradient (AG) transport lattice. Aberrations in the matching section cause further distortions of phase space.

Two distribution functions were used as the initial distribution (initialized after the matching section) for the simulations in this paper. A semigaussian distribution (uniform in density, Maxwellian in velocity with uniform temperature given by the beam emittance), was used to study the dynamic aperture. Calculations were then done for comparison, using a distribution calculated by simulating from the emitter, using space-charge-limited injection, through the injector and matching section. This self-consistent beam thus begins the simulation with the density oscillations and aberrations from the injector and matching system described above. Figure 2 shows the 2D
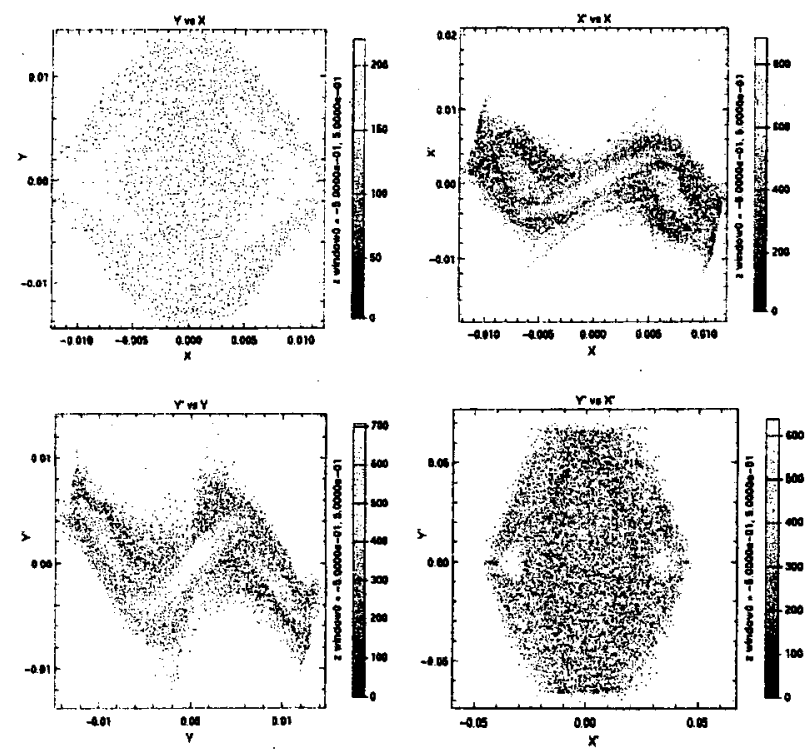

spatial and phase space projections for this initialization Figure 2. Configuration and phase space distributions for the beam simulated from the emitter, shown at the entrance to the AG transport section of HCX.

of the beam, which will be referred to below as the "more realistic" beam distribution, at the entrance to the AG lattice. The average $\mathrm{xx}^{\prime}$ of the beam (where ' $=\mathrm{d} / \mathrm{dz}$ ), which gives it a large slope in phase space, has been removed so that details of the distribution can be seen. The same has been done for yy'. It should be noted that the aberrations seen in these simulations have led to a probable change in HCX parameters-- it is likely that the current will be decreased to $600 \mathrm{~mA}$ to decrease envelope size in the injector and matching section, decreasing nonlinearities sampled by the beam. The normalized emittance (normalized edge emittance, i.e., $4 \mathrm{x}$ ms. will be used throughout this paper) chosen for the semigaussian was $6.3 \pi \mathrm{mm}$-mrad, which is four times the thermal emittance at the source (assuming $0.1 \mathrm{eV}$ ion temperature), and close to the emittance of the more realistic distribution.

\section{EFFECT OF NONLINEAR FOCUSING FIELDS AND IMAGE FORCES}

We will discuss in this section primarily simulations done for an undepressed phase advance per lattice period of $\sigma_{0}=60^{\circ}$. Simulations were done for $\sigma_{n}$ values from $45^{\circ}$ to $80^{\circ}$, but $60^{\circ}$ is the phase advance at which particle loss began $(0.3 \%$ loss $)$, so, as discussed below, this case can be considered as defining the dynamic aperture. At this focusing strength the maximum matched rms beam radius is $1.8-1.9 \mathrm{~cm}$, depending on the distribution function, or about $80 \%$ of the quadrupole aperture. Simulations for the semigaussian were first done with only the quadrupole moment of the focusing fields included. The aperture was also increased to 2.4 times the initial beam radius at the center of a drift, in order to effectively eliminate image forces. The beam was matched to the transport lattice using an envelope code which included only the quadrupole moment (as a function of $z$ ) of the 3D field decomposition. This produced a nearly negligible mismatch in rms radius of $+1-0.5 \%$. The rms emittance was essentially constant- it decreased slightly $(\sim 1 \%)$ during transport through 50 lattice periods, an effect well known for intense beams as the semigaussian relaxes.

Image forces were then added by placing the conducting boundaries of the circular quadrupole electrodes at the proper location. Inclusion of images caused the mismatch to increase to $+/-3 \%$. The beam shape began to oscillate, assuming a diamond shape at some $\mathrm{z}$ locations, as the edges of the beam were pulled toward the electrodes. Rms emittance in the $x$ dimension increased initially by $0.1 \pi \mathrm{mm}$-mrad and then stayed constant. This level of increase is marginally measurable with present diagnostics, and is not significant for a driver.

When moments beyond the quadrupole were added, mismatch stayed about the same, showing that the effect of images dominates the effect of focusing nonlinearities in the mismatch. Emittance growth was also very similar to the previous case with image forces only- it is negligible- though with extra structure (peaks in emittance vs. $z$ ) at the $z$ location of the fringe fields. The visible effects of transport on the beam were the growth 
of a small ( $\sim \mathrm{few} \mathrm{mm}$ ) halo, and a few $\mathrm{mm}$ increase in the radius of the beam due to image forces.

As the value of $\sigma_{0}$ was decreased from $60^{\circ}$ to $45^{\circ}$, increasing the size of the beam, the particle loss increased from $0.3 \%$ to $2 \%$ at $55^{\circ}, 13 \%$ at $50^{\circ}$, and $24 \%$ at $45^{\circ}$. Since fields at the edge of the aperture are not adequately modeled in this moment approximation, the exact amount of particle loss is probably not accurate. But it is notable that rms emittance growth remained negligible, showing that halo particles were quickly lost, with their large rms emittance contribution. In sum, the dynamic aperture seems to be evinced by particle loss, which occurs before any significant emittance changes. In reality, but not represented in the simulation, loss of heavy ions will produce electrons, neutrals, and ions which will interfere with beam propagation. Thus the particle loss we see in the calculations is likely to indeed be the aperture-limiting effect. We note that the particle loss is a gradual function of beam radius.

\section{DISTRIBUTION FUNCTION EFFECTS}

Transport of the more realistic beam distribution described above through 50 lattice periods was also simulated, again for the $\sigma_{0}=60^{\circ}$ case, to explore the effect on the HCX experiments of the upstream beam aberrations. Beam loss and mismatch were very similar for the semigaussian and the more realistic beam. There was a slight $(\approx 0.2 \pi \mathrm{mm}$-mrad) increase in the normalized emittance in one plane. The final phase spaces for the two beams are quite different, demonstrating that, as expected, the effects of the initial distribution function persist. The $x-x^{\prime}$ phase spaces for the two cases are shown in Fig. 3. As in Fig. 2, the average $x x^{\prime}$ of the beam has been removed. From this figure one can see that the more realistic beam has a lower temperature core, with more of the beam in halo. The halo in configuration space is approximately twice the width found for the semigaussian. For the case of $\sigma_{0}=80^{\circ}$ with the more realistic beam, the halo is larger still, $(\approx 5 \mathrm{~mm})$, though the beam radius is much smaller (maximum matched radius is $1.6 \mathrm{~cm}$ ). Thus the halo seems to be caused by mismatch and density oscillations, rather than to any aperturelimiting effect. Both semigaussian and more realistic beams have shapes that oscillate from diamond to elliptical due to

image forces. Much more simulation is needed to confirm and explain what is seen here, but it appears that the HCX will be able to study halo formation from mismatch and density oscillations, and that the real beam could have similar particle loss aperture limitations to the idealized semigaussian.

\section{QUADRUPOLE MISALIGNMENT AND ROTATION ERRORS}

Simulations were also performed with a semigaussian initial distribution for beams initialized at the center of a drift with centroid angles of 6 and 12 milliradian, modelling misalignments. We describe here runs for $\sigma_{0}=60^{\circ}$ with initial centroid velocity in the $x$ direction. The beam centroid reached a maximum distance from the channel center of 4 and $7 \mathrm{~mm}$ respectively for the 6 and $12 \mathrm{mrad}$ cases. The emittance change was negligible for both, but changes in the phase space were evident and should be measurable. The beam edge toward the electrode was slightly pointed due to images. Particle loss was $1 \%$ and $15 \%$ respectively, after 20 lattice periods.

Rotations of the first quadrupole, which can be rotated in the experiment, were also studied briefly. A rotation of 4 degrees can be seen in the rotation of the diamondshaped shape of the beam downstream, with attendant rotation in phase space. There was a $0.7 \pi \mathrm{mm}-\mathrm{mrad}$ increase in the generalized emittance, $\varepsilon_{\mathrm{g}}[5]$.
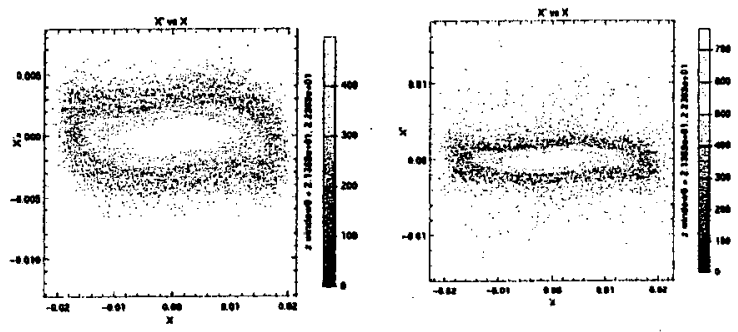

Figure 3. $x-x^{\prime}$ phase space for the semigaussian (left) and more realistic distribution function at the end of 50 periods of transport. $\sigma_{0}=60^{\circ}$.

\section{SUMMARY}

2D PIC simulations indicate that the signature of the dynamic aperture limit in the HCX is particle loss, rather than detectable changes in emittance. Halo formation through mismatch and density oscillation appears to be measurable. Misalignment or rotation of the quadrupoles causes changes in beam shape and orientation, but again, changes in rms emittance due to these mechanisms appear undetectable, at least in the length of the $\mathrm{HCX}$ electrostatic lattice.

\section{REFERENCES}

[1] David P. Grote, A. Friedman, I. Haber, W. Fawley, J. L. Vay, "New Developments in WARP3d: Progress Toward End-to-End Simulation", Nuclear Inst. and Methods in Physics Research, A, 415, Nos 1, 2, p $428,1998$.

[2] V. Karpenko et al., "An Engineering Overview of an Electrostatic Quadrupole Lattice for a High Current Transport Experiment", these proceedings. 
[3] F. M. Bieniosek $\dagger$, E. Henestroza, J. W. Kwan, L. Prost, P. Seidl, "2-Mv Injector For Hcx", these proceedings.

[4] E. Henestroza, F. Bieniosek, J. Kwan, A. Friedman, D. Grote, "Beam Dynamics Studies of the Injector and Matching Section for HCX", these proceedings.

[5] R. A. Kisheket al., "Effects of Quadrupole Rotations on the Transport of Space-Charge-Dominated Beams" Proc. of the Particle Accelerator Conference, March - 2 April, 1999, New York, NY, 1761. 
Submitted for publication to Physics of Plasmas

\title{
Progress in Heavy Ion Fusion Research
}

C.M. Celata, F.M. Bieniosek, E. Henestroza, J.W. Kwan, E.P. Lee, G. Logan, L. Prost, P.A. Seidl, J-L. Vay, W.L. Waldron, and S.S. Yu

Lawrence Berkeley National Laboratory, 1 Cyclotron Road, Berkeley, CA 94720

J.J. Barnard, D.A. Callahan, R.H. Cohen, A. Friedman, D.P. Grote, S.M. Lund, A.

Molvik, W.M. Sharp, and G. Westenskow

Lawrence Livermore National Laboratory, 7000 East Ave., Livermore, CA 94550

Ronald C. Davidson, Philip Efthimion, Erik Gilson, L.R. Grisham, Richard Majeski, Hong Qin, and Edward A. Startsev

Princeton Plasma Physics Laboratory, P.O. Box 451, Princeton, NJ 08543

S. Bernal, Y. Cui, D. Feldman, T.F. Godlove, I. Haber, J. Harris, R.A. Kishek, H. Li, P. G. O'Shea, B. Quinn, M. Reiser, A. Valfells, M. Walter, and Y. Zou

Institute for Research in Electronics and Applied Physics, University of Maryland, College Park, MD 20742

D.V. Rose and D. R. Welch

Mission Research Corporation, Albuquerque, NM 87107

\begin{abstract}
The U.S. Heavy Ion Fusion program has recently commissioned several new experiments. In the High Current Experiment [ P.A. Seidl et al., "The High Current Experiment: First Results", accepted for publication in Laser and Particle Beams 20 (4), 2002], a single low-energy beam with driver-scale charge-per-unit-length and space charge potential is being used to study the limits to transportable current posed by nonlinear fields and secondary atoms, ions, and electrons. The Neutralized Transport Experiment similarly employs a low-energy beam with driver-scale perveance (dimensionless perveance $=$ space potential energy/beam kinetic energy) to study final focus of high perveance beams and neutralization for transport in the target chamber. Other scaled experiments-- the University of Maryland Electron Ring [P.G. O'Shea. et al., accepted for publication in Laser and Particle Beams 20 (4)] and the Paul Trap Simulator Experiment [R.C. Davidson, H. Qin, and G. Shvets, Phys. of Plasmas 7, 1020 (2000)]-- will provide fundamental physics results on processes with longer scale lengths. An experiment to test a new injector concept is also in the design stage. This paper will describe the goals and status of these experiments, as. well as progress in theory and simulation. A proposed future proof-of-principle experiment, the Integrated Beam Experiment, will also be described.
\end{abstract}

\section{Introduction}

The goal of the U.S. heavy ion fusion (HIF) program is to produce commercial electricity by using multiple beams of heavy ions ( $\mathrm{A} \sim 100-200 \mathrm{amu}$ ) at a few $\mathrm{GeV}$ to implode an inertial fusion target. In order to achieve this goal, space-charge-dominated beams must be accelerated and compressed to form $\sim 10$ ns pulses, then focused and propagated through the target chamber to the target. The target chamber first wall is protected from radiation, neutrons, and debris from the fusion reaction by liquid jets of 
the molten salt FLiBe. As the beam propagates through the chamber, the charge state of the ions will be changed by several competing processes: stripping and neutralization due to FLiBe vapor, and photoionization from target $\mathrm{x}$-rays. Much of the physics of beam propagation from source to target has already been explored in a series of experiments using low-energy beams, with parameters scaled to produce the space-charge-dominated regimes of the fusion driver. Stable transport ${ }^{1}$, acceleration and compression ${ }^{2}$, beam combining $^{3}$, and final focus ${ }^{4}$ have been studied in this way. However these experiments were unable to study several important phenomena, among them electron effects such as neutralization (which requires a beam with driver-like current and beam perveance, i.e., ratio of beam space charge potential energy to kinetic energy), and long spatial-lengthscale phenomena (e.g., longitudinal wave physics, where the growth length of perturbations is longer than present experiments).

The present program is configured to address many of these issues. The High Current Experiment ${ }^{5}$ (HCX) is using a beam of charge per unit length characteristic of the lowenergy end of a driver to to study transport limits. The Neutralized Tranport Experiment (NTX) is using a low-energy beam with a perveance characteristic of the high-energy end of a driver to study final focus and neutralization. Other scaled experiments are being used to study long-length-scale phenomena. The University of Maryland Electron Ring ${ }^{6}$ (UMER) achieves long path length by recirculating particles in a ring, while the Paul Trap Simulator Experiment ${ }^{7}$ (PTSX) confines particles for long times. Finally, a new concept has emerged for a "multibeamlet" heavy ion injector which, if successful, is expected to decrease injector size and cost. An experiment is being designed to test this concept. The status of all of these experiments will be described in Section II.

Due to the competing processes of neutralization and stripping described above, beam propagation in the target chamber encompasses perhaps the most complicated physics of the heavy ion driver. Recent simulation results show focusing adequate for the driver for both the initial low energy beams which begin the compression, and for the main pulse beams, for bismuth ions. These simulations are described in Section III.

When present experiments have concluded, many heavy ion fusion driver issues will have been investigated in experiments tailored to study relevant physics for a given section of the driver. However, there remains a need to integrate the physics into a source-to-target experiment. Two experiments (the Scaled Final Focus Experiment ${ }^{4}$ and the NTX), for instance, will have studied aspects of final focus physics, including aberration correction and neutralization at high perveance, but in both experiments the beam was focused directly after matching from the source, rather than, as in a driver, after transport with acceleration followed by longitudinal compression. Another experiment is required which would integrate the source-to-focus physics into a sequence, thus giving an approximation of the correct distribution function at the final focus system. This integrated proof-of-principle experiment, the Integrated Beam Experiment (IBX), is in the initial physics design stages now. Important physics which could not be studied in previous experiments is also included in its mission, namely, longitudinal drift compression, bending of space-charge-dominated beams, and some longitudinal beam physics experiments. The IBX will be described in Section IV.

\section{The Present Heavy Ion Fusion Experimental Program}

\section{A. The High Current Experiment}


The mission of the High Current Experiment is to explore the physics which limits the amount of beam that can be transported through an accelerator of a given aperture. This limitation on transport efficiency affects the eventual cost of a heavy ion fusion driver, and also determines the optimal aperture for future experiments. Particlein-cell simulations predict that the "dynamic aperture" (i.e., the usable aperture as defined by beam dynamics) for the case of a perfectly aligned beam is determined only by the limitation of physical scraping of the beam at the aperture ${ }^{8}$. The High Current Experiment is testing this conclusion at beam intensities and pulse durations characteristic of those envisioned in HIF drivers near injection energy. It also will explore the effects of misaligned beams, distributions with nonuniform density, and of beam envelopes mismatched to the focusing system, which are expected to produce beam halo.

A $0.18 \mathrm{~A}, 4 \mu \mathrm{s}, 1 \mathrm{MeV} \mathrm{K}{ }^{+}$beam is presently being used for the dynamic aperture studies. Following a 6-quadrupole matching system, the beam is transported through a periodic alternating-gradient focusing system consisting of 10 electrostatic quadrupoles with $23 \mathrm{~mm}$ aperture. The beam has been aligned with the focusing channel to within 0.5 $\mathrm{mm}$ and $2 \mathrm{mrad}$, and its envelope matched to within $2 \mathrm{~mm}$. When the beam is matched to fill $60 \%$ of the $23 \mathrm{~mm}$ radial aperture, no emittance growth (i.e., no beam heating) is observed during transport, in agreement with simulation. Subsequent experiments will increase the beam radius until degradation of beam quality occurs. Beam halo production, and desorption of gas from the walls as beam particles scrape the vacuum wall, will be monitored.

Though the fields of electrostatic quadrupoles will sweep out electrons, in magnetic quadrupoles, which make up most of the transport system of the driver, electrons can persist and affect beam transport. Electrons produced by beam halo ions scraping the vacuum wall, or by ionization of background gas, will change the space charge of the beam, adversely affecting focusing, and perhaps even producing instability.

Experiments beginning now on the HCX will provide data to determine how large the beam pipe aperture must be in order that these effects be kept negligible. First the secondary electron coefficient will be measured. Very little experimental data is available on the secondary electron and atom coefficients for the case of heavy ions at grazing incidence, the case most relevant to the driver, and of course the coefficient will vary with surface material and preparation. Preliminary HCX measurements of the electron coefficient are shown in Fig. 1. For these measurements the beam has been aimed at various angles to a metal plate. The data follow the $\cos (\theta)$ dependence known from the literature, and show a secondary electron coefficient which varies from 20 at $15^{\circ}$ to 100 at $2^{\circ}$ from grazing incidence. The distribution of electrons in the beam will be measured in an alternating gradient lattice of four pulsed quadrupole focusing magnets, which are instrumented to detect electrons and desorbed gas. These are ready to be installed at the end of the HCX transport lattice. The currents of electrons and ions produced by beam halo scraping the quadrupole surfaces, and by background (and desorbed) gas ionization, will be measured. Measurements of the ion distribution function, which gives the beam space-charge potential, can be used to calculate the radius at which the electron-ion pair was "born", and then the density of electrons vs. radius can be calculated, with the help of particle simulation. Provision has been made to add induction cells in the gaps between quadrupole magnets to in order to study the effect of 
acceleration on electron accumulation and dynamics. Prototype superconducting quadrupoles have been built, and will also be installed in the HCX beamline to further the research into electron dynamics and gain operational experience with the focusing technology appropriate for most of a driver.

Extension of the HCX beamline by 20-30 electrostatic quadrupoles is also planned. This would allow more precise measurements of transport effects, which evolve over comparable lengths.

B. The Neutralized Transport Experiment

The NTX consists of a $400 \mathrm{keV}$ ion diode, followed by a 4-quadrupole magnetic final focus system, and $1 \mathrm{~m}$ of drift in which neutralization experiments will be performed. The beam is an $80 \mathrm{~mA} \mathrm{~K}^{+}$beam. Aperturing is done in order to vary the perveance and to improve beam quality by clipping off beam affected by diode aberrations. Particle-in-cell simulations using the LSP $\operatorname{code}^{10}$ have shown that neutralization caused by passing the beam through a plasma can greatly improve the beam focus ${ }^{11}$. In a driver this can be done by inserting a "plasma plug", with a density $\left(\sim 10^{13} \mathrm{~m}^{-3}\right)$ which is significantly higher than the beam density, into the beamlines after the final focus system. Two other sources of electrons are available to neutralize the beam: electrons produced by beam ionization of FLiBe vapor in the chamber, and, after ignition, electrons in plasma formed around the target by photoionization of FLiBe vapor by fusion $\mathrm{x}$-rays. Both of these mechanisms will be simulated in the NTX. A pulsed metal plasma arc source of length $10 \mathrm{~cm}$ and $>10^{10} \mathrm{~cm}^{-3}$ density is located $20 \mathrm{~cm}$ from the last focusing lens, to simulate the plasma plug. A $10-\mathrm{cm}$-long ECR plasma source providing a $10^{12} \mathrm{~cm}^{-3}$ argon or helium plasma will be installed soon farther downstream. The spatial density profile from this source can be tailored by varying the axial profile of a solenoidal field around the beam pipe, in order to simulate the spatial distribution expected for the photoionized plasma.

Construction of the NTX has just been completed, and machine commissioning is in progress. Early data from the NTX showing a large improvement in focusing when the plasma plug is present is shown in Fig. 2.

Optimization of the focus of the 4-quadrupole final focus lens system, including minimization of higher-order aberrations, will also be studied in the coming months on the NTX. In the future, octupole windings may be inserted in the bore of the focusing system magnets for studies of the correction of $3^{\text {rd }}$ order aberrations. Though aberration correction is well-understood in other optical systems, techniques are largely untested for high perveance beams.

C. Small, Scaled Experiments

Two university experiments are beginning fundamental studies in intense beam physics important to Heavy Ion Fusion. One is the University of Maryland Electron Ring, and the other is the Paul Trap Simulator Experiment at Princeton Plasma Physics Laboratory. In both of these experiments long-length-scale phenomena can be studied, because the particles are confined over many betatron periods. In UMER this is done by circulating the beam in a small fast-cycling synchrotron. The PTSX confines ions in a Paul trap for long times ( 45 seconds), and time-dependence of the voltage on the radialconfinement electrodes simulates the passage of focusing quadrupoles in the "beam frame". Both experiments are in the beginning stages of operation. They are described below. 
1. The University of Maryland Electron Ring

UMER uses a non-relativistic electron beam to simulate intense ion (and electron) beam physics in a compact ( $11.52 \mathrm{~m}$ circumference) experiment. The accelerator is designed to have access to a wide range of beam parameters. The phase advance per focusing period of the transverse oscillation of a particle in the focusing field, $\sigma$, is the "depressed betatron phase advance". The ratio of this number to the value of the phase advance neglecting space charge, the "undepressed betatron phase advance", $\sigma_{0}$, is a measure of the influence of space charge on the beam. In UMER, $\sigma / \sigma_{0}$, can vary from 0.2 to 0.8 , a range which takes dynamics from emittance-dominated to space-chargedominated. The input beam current is variable from 0.4 to $100 \mathrm{~mA}$, giving a generalized perveance of $0.0006-0.0015$. At $10 \mathrm{keV}$ the normalized emittance for $100 \mathrm{~mA}$ is 15 $\mathrm{mm}$-mrad. The pulse length can be varied from 40 to $100 \mathrm{~ns}$. These parameters overlap the heavy ion fusion regime. At present, 18 focusing quadrupoles out of 72 have been installed in the ring. The ring is expected to be completed in the summer of 2003. The $100 \mathrm{~mA}$ beam shape in the injector, as measured by a phosphorescent screen, is shown in Fig. 3.

When the ring is completed, multi-turn operation will be attempted, the goal being to attain 100 turns at low current $(10 \mathrm{~mA})$ and 10 turns at high current $(100 \mathrm{~mA})$, with final emittance within a factor of 4 of the initial value. Subsequent upgrade of UMER would allow for ramping the dipoles, to enable acceleration of up to $2 \mathrm{keV}$ per turn.

2. The Paul Trap Simulator Experiment

In the Paul Trap Simulator Experiment, Cesium ions with density $10^{6} \mathrm{~cm}^{-3}$ are injected into a gold-plated metal cylindrical vacuum chamber. The conducting walls are split into four electrodes (see Fig. 4) which are charged as shown in the figure. At the "beam", whose radius $\left(r_{p} \sim 1 \mathrm{~cm}\right)$ is much less the $10 \mathrm{~cm}$ wall radius, $r_{w}$, the field is that of a quadrupole, with small corrections of order $\left(r_{p} / r_{w}\right)^{4}$. The wall voltage, $V_{0}$, oscillates at $100 \mathrm{kHz}$, simulating passing quadrupoles of a transport system as seen in the beam frame. Waveforms can be varied to explore the effects of different variations of quadrupole field with $\mathrm{z}$. Positive voltage on the cylinder endplates provides longitudinal confinement. The end-to-end bounce time in the $2 \mathrm{~m}$-long experiment is much smaller than the betatron period (i.e., the period of the transverse oscillation of the particles due to the transverse focusing), minimizing the effect of the ends on dynamics. The long ( $45 \mathrm{~s})$ confinement time makes possible the study of slow-growth-time phenomena. The experiment will be used to study beam mismatch, envelope instabilities, halo production, beam compression techniques, longitudinal wave physics. and beam profile effects.

Experiments on the PTSX began in June of 2002. Figure 5 is early data showing the current produced by the source as a function of $V_{0}$ and wall voltage frequency. Longitudinal confinement is not provided for this experiment-- the ions are freestreaming longitudinally. The calculated area of known envelope instability is to the left of the white parabola in the figure, and its effect on ion density can be seen in the contours of current measured by a Faraday cup at the end of the experiment. The change in ion profile as the instability is approached is also shown in the figure.

D. Multibeamlet Injector Test Experiments

As the voltage on a diode is increased, in order to increase the current generated, its length must increase in order to avoid electrical breakdown. The Child-Langmuir equation, combined with voltage breakdown scaling, shows that if the ratio of the length 
of a diode to its diameter is held constant in order to maintain good optics, the current density must decrease as voltage and total current increase. Thus beams of smaller diameter produced by a diode have higher current density than large-diameter beams. This offers the opportunity of making a more compact diode and injector (the accelerator section directly after the diode) if each accelerator beam can be made by merging smalldiameter bright beamlets. Another advantage of this merging minibeamlet approach is the possibility of greatly reducing the size of the "matching section". In the conventional accelerator design, a matching section with diameter larger than that of the injector is needed to transform the round-cross-section of beams emitted from the diode to the elliptical cross-section required for the alternating gradient focusing system of the accelerator. The diameter of this section can be substantially reduced with the "multibeamlet" approach, since the merging beamlets can be arranged in an elliptical pattern of the correct size for the accelerator. A potential problem is the emittance growth inherent in the merging process. Two and three-dimensional particle-in-cell simulations of the merging process ${ }^{12}$, using the WARP code ${ }^{13}$, produce a final transverse emittance of 0.8 to $1.4 \pi \mathrm{mm}$-mrad. While this emittance is approximately double that from a conventional single emitter diode, it is within acceptable limits for current driver designs. The final emittance shows a very weak dependence on the source temperature, allowing considerable flexibility in the ion source choice Figure 6 shows the evolution of the beam density and phase space. Within $40 \mathrm{~m}$, it approaches uniform density, with acceptable uniformity in phase space.

An experimental test of this concept is in the design stage. In the first stage, in FY03, approximately $1005 \mathrm{~mA}, 80 \mathrm{keV} \mathrm{Ar}{ }^{+}$beamlets will be produced by placing a multi-aperture extraction diode in from of a plasma ion source. The beamlets will propagate in parallel. Plates perpendicular to the direction of travel, with apertures for each beamlet, will be biased in order to provide Einzel lens focusing. Beamlet alignment, electron and gas production, and beam quality will be measured. In the second stage, an experiment on the LLNL $500 \mathrm{kV}$ test stand will accelerate the beamlets to $500 \mathrm{keV}$ and merge them. Emittance growth due to merging, alignment, and phase and spatial profiles of the final merged beam will be measured. If successful, this concept could lead to a lower-cost, smaller-diameter, lower-weight multibeam injector.

\section{Neutralization and Chamber Propagation Simulation Results}

An extensive theory and simulation effort supports the above-described experiments, and explores driver issues. Because of space limitations, only one important highlight of this effort is described here. For overviews of HIF theory please see the following recent reviews: D. C. Davidson et al., "Overview of Theory and Modeling in the Heavy Ion Fusion Virtual National Laboratory", accepted for publication by Lasers and Particle Beams; and D. R. Welch, D. V. Rose, B. V. Oliver, T. C. Genoni, R. E. Clark, C. L. Olson, and S. S. Yu, Phys. Plasmas 9, 2344 (2002).

The LSP code, a 3-D multi-species electromagnetic particle-in-cell code, has been used for axisymmetric simulations of heavy ion beam propagation through the target chamber. Many complicated effects were included in the simulations: neutralization by plasma injected into the beampipe just upstream of the chamber entrance, by electrons emitted by the conducting wall near the entrance, and by those from collisional ionization by the beam of FLiBe vapor; beam stripping by FLiBe vapor and by $\mathbf{x}$-rays from the target; and beam neutralization and stripping by the plasma formed by photoionization of 
vapor around the target. Beam parameters were chosen to match a recent version of the distributed-radiator indirect-drive target introduced by Tabak and Callahan ${ }^{14}$, which requires $6.5-7 \mathrm{MJ}$ to be deposited within an annulus of half-width $2.2 \mathrm{~mm}$ at each end of the target. This means that $90-95 \%$ of the energy of each beam must focus in an circle of radius $2.2 \mathrm{~mm}$. The correct energy deposition time profile for target compression is produced by phasing the arrival of beams of different energy, as sketched in Fig. 7. Focusing is most difficult for the earliest beams to arrive (the so-called "foot pulse" beams), and for the shorter of the main pulse beams. The earliest foot beams arrive before the target is heated sufficiently to photoionize the nearby FliBe vapor, so these pulses must reach the target without neutralization by photoionization electrons that are available to later pulses, while the shorter main pulse beams have the highest current and perveance, and therefore the largest space charge forces before neutralization.

Parameters for these two types of beam were used in the simulations described below.

LSP calculations ${ }^{11}$ were done with a $7 \times 10^{12} \mathrm{~cm}^{-3}$ density of $\mathrm{BeF}_{2}$ in the chamber, This density is appropriate for the thick liquid wall (i.e., FLiBe jet) chamber. $\mathrm{BeF}_{2}$ is the dominant gas component of FLiBe vapor and has been shown by simulations to adequately represent the vapor. For the $9.3 \mathrm{~ns}, 2 \mathrm{kA}, 4 \mathrm{GeV}$ main pulse $\mathrm{Bi}^{+}$ions, less than $1 \%$ fell within the required $2.2 \mathrm{~mm}$ radius spot when no plug plasma or photoionized plasma was present. Adding at each end of the $3 \mathrm{~m}$-long beam port a layer of plasma 10 $\mathrm{cm}$ thick (with $3 \mathrm{~cm}$ parabolic density falloff at the ends of each plasma), increased this percentage to $92 \%$ when the ionization length of the plasma was of the order of the distance to the target. This value rose to $94 \%$ when the effects of neutralization by the photoionized plasma were included. Beam focusing was insensitive to the length and density of the injected plasma layers so long as enough plasma electrons were available to neutralize the beam. However the scale length for the density falloff at the upstream end of the first layer was found to affect the emittance (transverse temperature) and therefore the focal spot of the beam. More gradual profiles decreased the rms spot radius by decreasing the electron current directed into the beam from the head as the beam reached the plasma.

Foot pulse focusing was also greatly improved by the addition of the preneutralizing plasma plugs. For a $1.5 \mathrm{kA}, 3 \mathrm{GeV}$ bismuth beam, $85 \%$ of the foot pulse focused within the required $2.2 \mathrm{~mm}$ radius. This value increased to $92 \%$ when the plasma density scale length was doubled. Thus focusing adequate for the driver was achieved for both foot and main pulse beams.

Changing the ion from bismuth ( $A=209 \mathrm{amu}$ ) to xenon (131 amu) decreased the percent of ions focused within $2.2 \mathrm{~mm}$ to $60 \%$ for the foot pulse, and $81 \%$ for the $9.3 \mathrm{~ns}$ main pulse. Using xenon is desirable because the lighter mass decreases the cost of the accelerator. Though adequate focusing has not yet been demonstrated for xenon, the system is not optimized, and it is likely that the spot size can be reduced.

\section{Plans for an Integrated Beam Experiment}

When the present experiments are completed, the HIF program will have experimentally demonstrated production and stable transport of intense beams, beam compression, limited acceleration and compression of multiple beams, beam combining, final focus with aberration correction, and beam neutralization. Many beam dynamics effects, including halo production and electron production and dynamics, will have been studied. The physics which will remain for future experiments includes bending of 
space-charge-dominated beams, the final drift compression, multiple beam physics (including interaction of the high-current beam bundle with the induction core impedance), very-long-length-scale physics such as longitudinal wave growth (which will be studied on the small-scale experiments, but must be repeated in the induction linac system) and high current/high energy physics, such as neutralization with non-negligible beam self-magnetic field. But along with exploration of these areas, it is also important to integrate into a sequential experiment the physics previously explored. While it was crucial to isolate the physics of each driver section in controlled, affordable experiments, the beam manipulations involved must now be integrated into a sequential system so that the evolution of the distribution function through all of the sections can be followed, and the physics of the individual sections can be studied with a distribution function which has been modified by previous sections, as in a driver.

The HIF program has begun to plan a proof-of-principle experiment, the Integrated Beam Experiment (IBX), which would investigate many of the remaining physics issues mentioned above, and which would also be an integrated test of source-tofocus beam physics. The IBX accelerator is at present envisioned to be a single beam $\mathrm{K}^{+}$ accelerator with final ion energy $\sim 5-10 \mathrm{MeV}$ and driver-scale perveance, variable between $10^{-5}$ and $10^{-3}$. To reduce cost, though the beam would be driver-scale in transverse dimension and charge-per-unit-length, the pulse length would be reduced to $\sim 0.2-1.5 \mu \mathrm{s}$ FWHM at injection (the driver pulse length at this energy $\sim 10$ 's of $\mu \mathrm{s}$ ).

Flexibility is being designed into the accelerator so that a range of acceleration and compression schedules can be examined. Experiments on longitudinal wave propagation and growth, beam compression during acceleration, drift compression (up to the factor of $\sim 20$ needed in the driver), beam bending, and the effect of electrons on ion transport will be possible, along with studies of integrated drift compression/final focus/neutralization/chamber transport. The IBX would be a large step forward. However, the separate pieces of physics, of beam acceleration, focusing, and compression, needed for the IBX have been demonstrated in small-scale experiments, and the technology, including superconducting magnets, will be prototyped in the HCX and in offline experiments. Present experiments, especially the HCX, NTX, and multibeamlet injector, will provide information which will optimize the design of the IBX and all subsequent experiments. The dynamic aperture experiments on the HCX, for instance, will optimize the aperture diameter, and the NTX data will be used to optimize the magnet design for the final focus. But the design of the IBX can be done without this information, and later adjusted to the optimized values. Costing studies indicate that for a single-beam accelerator the effect of these changes on total cost would be minimal (e.g., $\sim 5 \%$ change in total cost for a $1 \mathrm{~cm}$ change in the $4 \mathrm{~cm}$ aperture). Construction of the IBX could begin in 2005 , if funding were available.

Multiple beam and high current/high energy beam dynamics, as well as verylong-length scale dynamics could not be studied in the IBX. Exploration of these issues would be the mission of the Integrated Research Experiment (IRE) - a several-hundred $\mathrm{MeV}$, multibeam, source-to-target experiment. The IRE would also be able to perform a limited set of experiments on heavy-ion-related target hydrodynamics, though most target issues would be tested on the National Ignition Facility.

\section{Conclusions}


In summary, four new experiments are beginning to produce data for the Heavy Ion Fusion program. They include experiments on transport limits (High Current Experiment ), and final focus and neutralization (Neutralized Transport Experiment), and small-scale experiments on long-length-scale issues and fundamental beam dynamics (the University of Maryland Electron Ring and the Paul Trap Simulator Experiment). A fifth experiment, which will investigate the feasibility of a new multibeamlet injector concept, will be in operation in mid-2003.

Along with these experiments, a program of simulation and theory serves to both support experimental data analysis and design, and explore driver issues which are not in reach of present experiments. Recent simulations have demonstrated focusing of both foot and main pulse beams onto the $2.2 \mathrm{~mm}$ spot required by present targets. Effects of ionization of the beam and ambient vapor by target $\mathrm{x}$-rays, neutralization by electrons emitted from the wall and from plasmas in the beam pipe, and stripping by $\mathrm{x}$-rays and background gas were included in the calculation. Preneutralization by plasmas in the beam port was especially important in the focusing process.

The present experiments will conclude a sequence of experiments that will have demonstrated most of the physics of the heavy ion driver. A proof-of-principle experiment, the Integrated Beam Experiment, is planned to succeed this phase. The IBX will demonstrate integrated source-to-focus transport, acceleration, compression, and focusing of a single beam, and will explore many of the remaining physics issues of heavy ion drivers, such as longitudinal dynamics, beam bending, and integrated drift compression/final focus/chamber transport.

\section{Acknowledgements}

This work was supported by the Office of Science, U.S.Department of Energy, under contract numbers DE-AC03-76SF00098, W-7405-Eng-48, DE-AI02-93ER40799, DEFG02-94ER40855 and DEFG02-92ER54178, and DE-AI02-94ER-54232.

\section{References}

${ }^{1}$ M.G. Tiefenback and D. Keefe, Trans. on Nuc. Sci. NS-32 (5), 2483 (1985).

${ }^{2}$ W.M. Fawley et al., "Beam dynamics studies with the heavy-ion linear induction accelerator MBE-4", Phys. Plasmas, 4(3), 880 (1997).

${ }^{3}$ P. A. Seidl et al., Nucl. Inst. Meth. A415, 243 (1998).

${ }^{4}$ S. A. MacLaren, A. Faltens, P. A. Seidl, and D. V. Rose, Phys. Plasmas 9, 1712 (2002).

${ }^{5}$ P.A. Seidl, D. Baca, F.M. Bieniosek, A. Faltens, S. M. Lund, A.W. Molvik, L.R. Prost, W.L. Waldron, "The High Current Experiment: First Results", accepted for publication in Laser and Particle Beams 20 (4), 2002. LBNL-50861.

${ }^{6}$ P.G. O'Shea. et al., accepted for publication in Laser and Particle Beams 20 (4), and Kishek et al., this issue.

${ }^{7}$ R.C. Davidson, H. Qin, and G. Shvets, Phys. of Plasmas 7, 1020 (2000).

${ }^{8}$ C.M. Celata, A.F. Friedman, D.P. Grote, I. Haber, and E. Henestroza, "Particle-in-Cell Simulations of Beam Dynamics in the HCX", accepted for publication in Laser and Particle Beams 20 (4).

${ }^{9}$ P. Thieberger, A. L. Hanson, D. B. Steski, et al., Phiys. Rev. A 61, 042901 (2000).

${ }^{10}$ T.P. Hughes et al., Phys. Rev. ST Accel. Beams 2, 110401(1999); D.R. Welch et al., Nucl. Instrum. and Meth. in Phys. Res. A 464, 134 (2001). 
${ }^{11}$ W.M. Sharp et al., "Simulation of Chamber Transport of Heavy-Ion Fusion", Proc. of $19^{\text {th }}$ IAEA Fusion Energy Conf., 14-19 Oct. 2002, Lyon, France, to be published.

12 Design and simulation of the multibeamlet injector for a high current accelerator", $D$.

P. Grote, E. Henestroza, and J. W. Kwan, submitted to PRSTAB

${ }^{13}$ David P. Grote, A. Friedman, I. Haber, W. Fawley, J. L. Vay, Nucl. Inst. and Meth. in Phys. Res. A, 415, Nos 1, 2, 428 (1998).

${ }^{14}$ D. A. Callahan-Miller and M. Tabak, Nucl. Fusion 39, 883 (1999); D. A. Callahan, M. C. Herrmann, and M. Tabak, "Progress in Heavy Ion Target Capsule and Hohlraum Design," accepted for publication in Laser and Particle Beams, 20 (4).

\section{Figures \& Captions}

Figure 1. Preliminary measurements of the electron emission coefficient, for $1 \mathrm{MeV} \mathrm{K}+$ incident on stainless steel, follow the $1 / \cos$ (theta) dependence between 75 and $87 \mathrm{deg}$. from normal incidence.

Figure 2. Beam profile ( $300 \mathrm{kV} 25 \mathrm{~mA}$ potassium ions) at the end of the plasma drift section for (a) plasma source turn plasma source off, and (b) plasma source on.

Figure 3 Photos of integrated beam cross-section in several planes of the injector, obtained using a Phosphor screen and a CCD camera, for the $100 \mathrm{~mA}$ intense beam; locations of screen: (a) $71 \mathrm{~cm}$, (b) $85 \mathrm{~cm}$, (c) $91 \mathrm{~cm}$, and (d) $103 \mathrm{~cm}$, all measured from the anode plane.

Figure 4. PTSX consists of 3 8-inch diameter cylinders, each divided into 490 -degree sectors. An AC voltage on the central cylinder confines the ions radially while a DC voltage on the end cylinders confines the ions axially. By varying the functional form of $\mathrm{V}_{0}(\mathrm{t})$, the effects of various quadrupole magnet configurations can be explored.

Figure 5. Steady state currents for ions traveling directly from the ion source to the Faraday cup. If the vacuum phase advance $\left(\sigma_{0}\right)$ is greater than 90 degrees (area to the left of the white parabola), the particle orbits are unstable and all ions strike the trap wall before reaching the Faraday cup.

Figure 6. PIC simulation of 91 beamlets $(0.5 \mathrm{~A}$ total) in a multibeamlet injector. Number density in configuration and phase space is shown beginning where the beamlets begin the merge. Final normalized emittance is jus under $1 \pi \mathrm{mm}-\mathrm{mrad}$.

Figure 7. Input-power profile delivered to the target by 120 constant-current beams with different durations and timings..

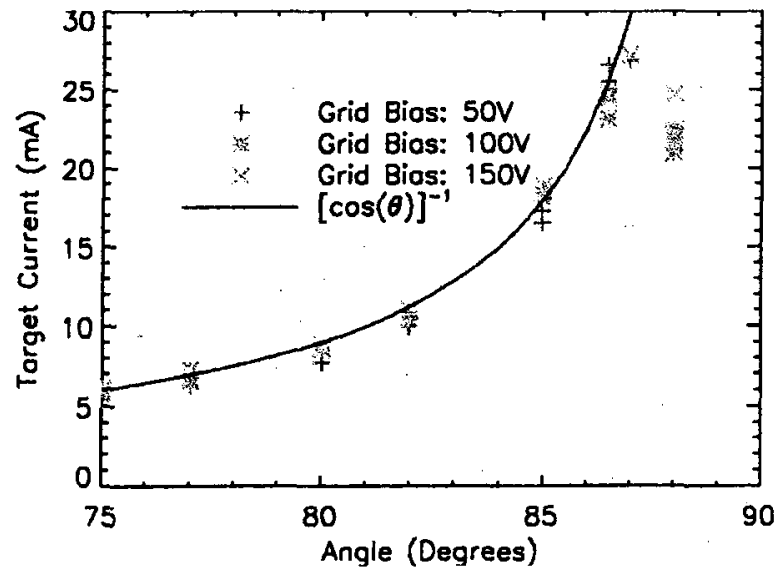


Figure 1
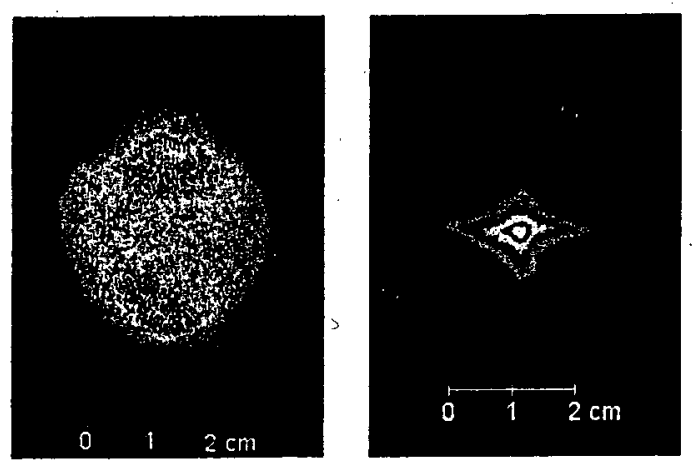

(a)

(b)

Figure 2
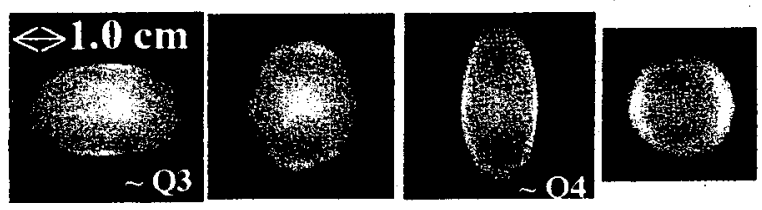

Figure 3
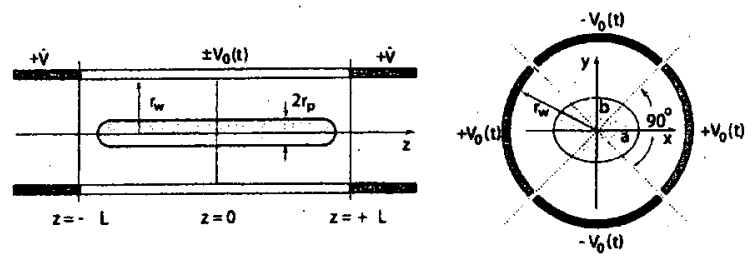

Figure 4 


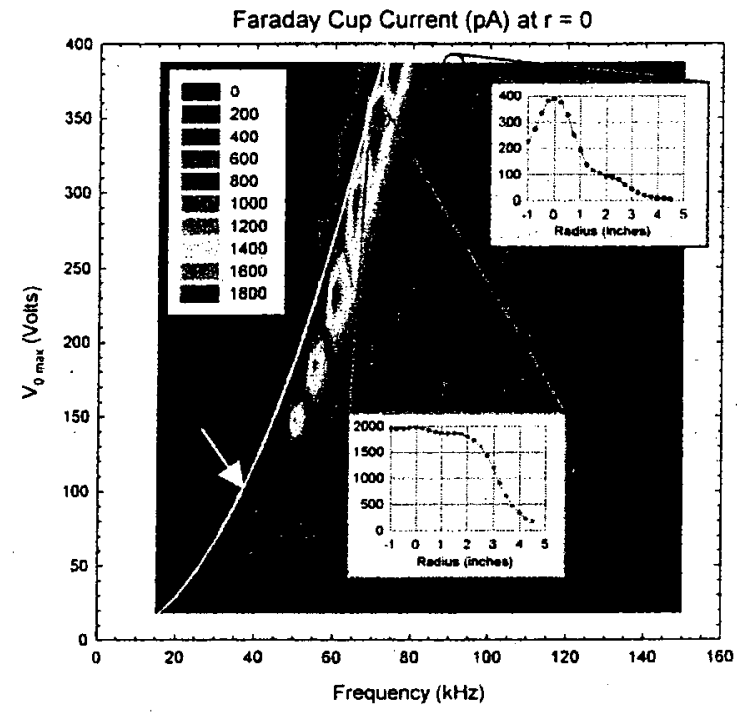

Figure 5

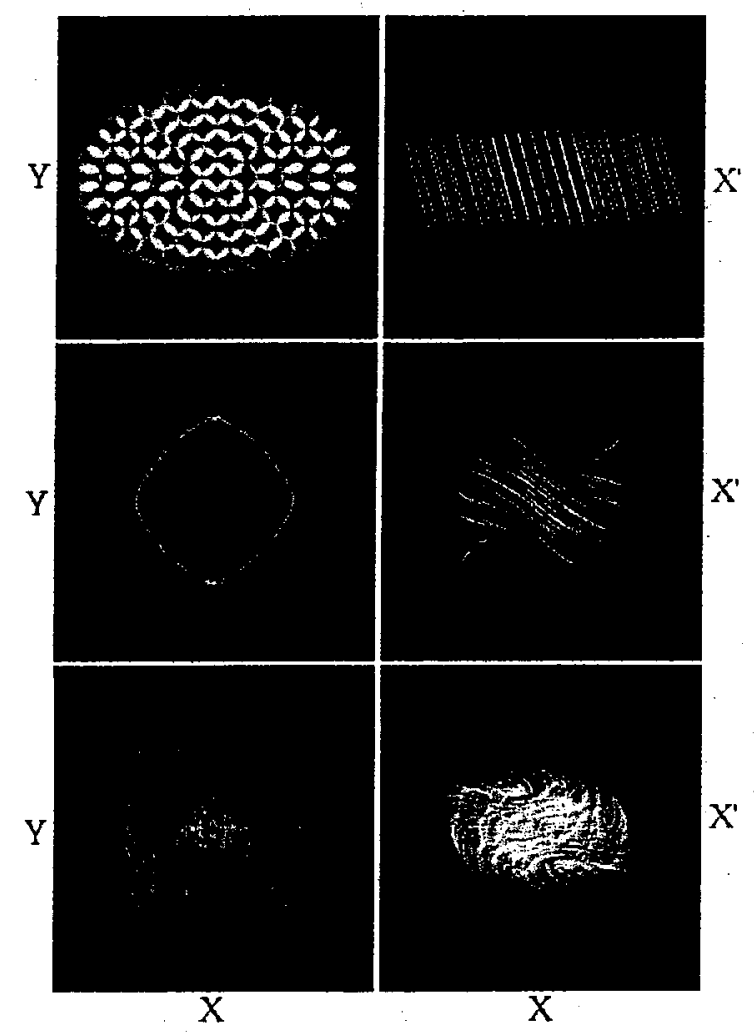

Figure 6 


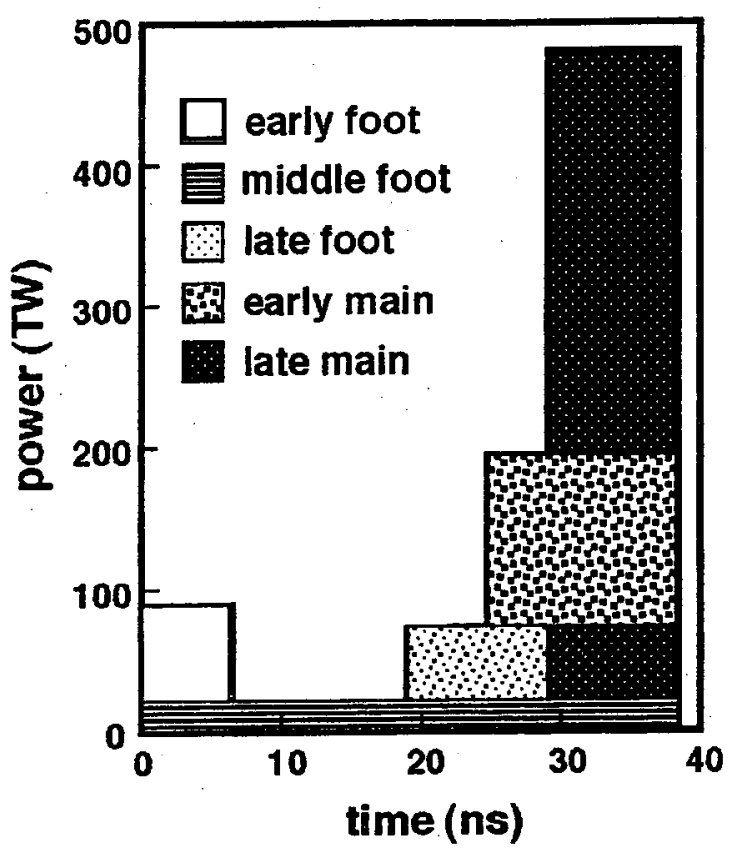

Figure 7 

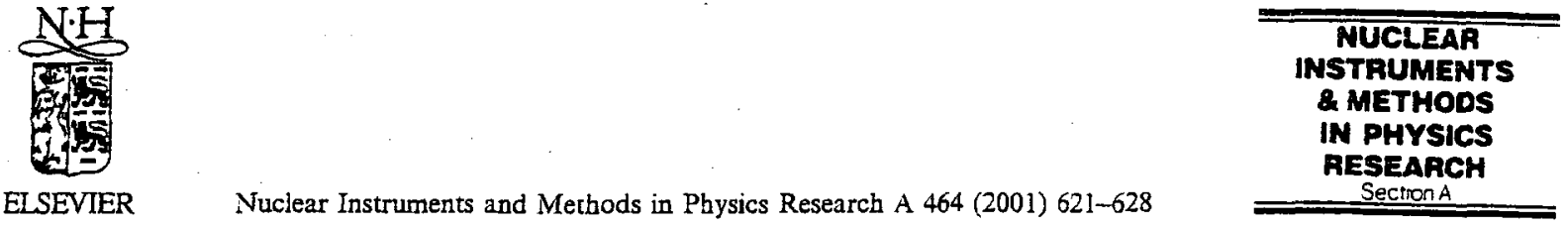

www.elsevier.nt/locate/nima

\title{
Planning for an integrated research experiment $t^{2}$
}

\author{
J.J. Barnard ${ }^{\mathrm{a}, *}$, L.E. Ahle ${ }^{\mathrm{a}}$, R.O. Bangerter ${ }^{\mathrm{b}}$, F.M. Bieniosek ${ }^{\mathrm{b}}$,
} C.M. Celata ${ }^{b}$, A. Faltens ${ }^{b}$, A. Friedman ${ }^{a}$, D.P. Grote ${ }^{a}$, I. Haber ${ }^{c}$, E. Henestroza ${ }^{b}$, M.J.L. de Hoon ${ }^{b}$, V.P. Karpenko ${ }^{a}$, R.A. Kishek ${ }^{d}$, J.W. Kwan ${ }^{b}$, E.P. Lee ${ }^{b}$,

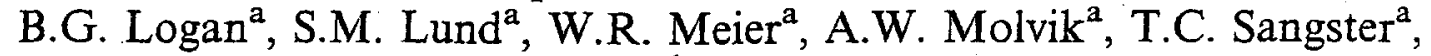
P.A. Seidl ${ }^{\text {b }, ~ W . M . ~ S h a r p ~}{ }^{\mathrm{a}}$

\footnotetext{
${ }^{a}$ Lawrence Livermore National Laboratory, P.O. Box 808, L-645, Livermore, CA 94551, USA

'Lawrence Berkeley National Laboratory. Berkeley, CA 94720, USA

${ }^{\circ}$ Naval Research Laboratory, Washington, DC 20375, USA

${ }^{d}$ University of Md., College Park, MD 20742, USA
}

\begin{abstract}
We describe the goals and research program leading to the Heavy Ion Integrated Research Experiment (IRE). We review the basic constraints which lead to a design and give examples of parameters and capabilities of an IRE. We also show design tradeoffs generated by the systems code IBEAM. (C) 2001 Elsevier Science B.V. All rights reserved.
\end{abstract}

P.ACS: $52.58 . \mathrm{Hm} ; 41.75 . \mathrm{A} ; 41.85 . \mathrm{Gy}$

Këywords: Füion; Heavy Ion; Inertial fusion; Driver; Accelerator; Systems model

\section{Introduction}

The Integrated Research Experiment (IRE), a major step in the US inertial fusion energy (IFE) program, will play a critical role in the development of IFE. The IRE must produce sufficient confidence to design and build an Engineering Test Facility (ETF), the final step towards an IFE

\footnotetext{
Wh Work performed under the auspices of the US Department of Energy under contract DE-AO33-76F0098 at University of California LBNL, contrast W-7405-ENG-48 at University of California LLNL, contract DE-A102-93ER40799 at NRL, and contract DE-FG02-92ER54178 at U. Md.

*Corresponding author. Tel.: +1-925-423-0675; fax: +1925-422-7390.

E-mail address: jjbarnard@llnd.goy (J.J. Bamard).
}

demonstration power plant. The heavy ion IRE conceptual design effort is projected to begin in about two years (Spring 2002), but preliminary design examples have already been generated as computational models to develop simulation tools, and to explore parameters for possible high energy density experiments.

In Section 2, we review the goals of the IRE, which set the scale for the project. In Section 3, we give a brief overview of the research program that is laying the scientific and technological groundwork for the accelerator. In Section 4, we outline the analysis leading to an example design which we are using as a testbed for our simulation tools. Finally, in Section 5 we give illustrative results from the systems code IBEAM. 


\section{Goals and scale of the IRE}

The overriding goal of the IRE, together with target results from the US Inertial Confinement Fusion program and progress on IFE chamber and target technology, is to provide the scientific and technological basis to proceed the ETF. The ETF will integrate all the major systems needed for an IFE power plant (driver, target production and injection, fusion chamber and heat removal). The ETF will demonstrate high rep-rate operation at low fusion yield (of order $30 \mathrm{MJ}$ ) and may include exploration of higher gain and yield targets. In order to meet the overriding goal, there are three general areas where the basis provided by the IRE must be solid: (1) Accelerator physics; (2) Chamber transport and final focus; and (3) Ion beam interaction with targets.

The accelerator physics basis includes all beam dynamics questions at driver scale, including emittance growth, halo formation, pulse compression, multiple beam effects, and "beam loading" (the effects of the beam itself on the driving circuitry, and ultimately back onto the beam). The IRE will also be, as its name indicates, an integrated system demonstration, from beam injector to target. Demonstrating the chamber transport and final focus basis for heavy ion fusion requires the ability to focus the beam at the target. This will entail testing several candidate methods for final focus including some that require beam neutralization. The IRE must also validate beam stability in the chamber (demonstrate absence of instabilities such as two-stream instability, filamentation, etc., or show that those that do exist are benign). Also, the effects of stripping of beam ions by chamber gas and photoionization of gas and beam by target $\mathrm{X}$-rays must be shown to be quantitatively understood. Finally, the IRE will study ion beam interaction with targets, including volumetric heating of matter (unique to ions) and ion stopping in dense plasmas, both of which are unobtainable from laser facilities, and needed to ensure target designs are well calibrated.

These overall goals lead to quantitative technical goals and an overall scale of the accelerator. Demonstration of an understanding of long-term transverse and longitudinal beam dynamics re- quires hundreds of lattice periods. In order for beam loading to have effects similar to those in a driver the total current at the end of the accelerator must be greater than of order $100 \mathrm{~A}$, a significant fraction of the total induction module current. This large total current will require tens of beamlets. To enable definitive focusing experiments from a variety of focusing modes, the ion energy must be greater than of order $100 \mathrm{MeV}$, the final generalized perveance must be at least that of a driver $\left(10^{-5}-10^{-4}\right)$, and the normalized emittance $\varepsilon_{\mathrm{N}}$ must be less than about $5 \pi \mathrm{mm}$ mrad. Validating beam-target interaction physics requires a flux greater than of order $3 \times 10^{12} \mathrm{~W} /$ $\mathrm{cm}^{2}$, and multi-kJ in the pulse, in order to heat matter to the $50 \mathrm{eV}$ range or greater. As the required capabilities of the beams are better understood, the exact scale of the accelerator will emerge.

Further, it would be desirable (but not essential) for the IRE to serve as the front end of the ETF. This may require reconfiguration (since near term goals may require lighter ion masses or different pulse durations than a driver), possible relocation, and durable components. Finally, final energies of bundreds of $\mathrm{MeV}$, and currents of tens of $\mathrm{A} / \mathrm{beam}$ at the end of the accelerator are required to meet the ultimate goal of providing a single intermediate step between the near term experiments ( $\sim$ few $\mathrm{MeV}, 1 \mathrm{~A} / \mathrm{beam})$ and the ETF ( $\sim$ few $\mathrm{GeV}$, $\sim 10^{2} \mathrm{~A} /$ beam at end of accelerator). A smaller scale would lead to a larger extrapolation from IRE to ETF.

\section{Overview of research program in preparation for an IRE}

The preparation for an IRE and long term driver feasibility research drive the near term research program. See Ref. [1] for a more detailed discussion of the US Heavy Ion Fusion Program. The current "Phase I" program has two new experiments among its research goals: (1) A highcurrent prototype injector module (leading to $10-100 \mathrm{~A}$, at about $1 \mathrm{~A} /$ beam, and leading to an IRE injector with a cost of less than of order $\$ 20 \mathrm{M}$ ) (cf. [2]); and (2) A high-current transport 
and focusing experiment $(\mathrm{HCX})(\mathrm{a} \sim 1 \mathrm{~A}$ single beam machine designed to study the transport limits of beams with driver-scale line charge density) (cf. [3]). The Phase I program also has an enabling technology component, that is focused on the development of four critical components: (1) Multiple-beam quadrupole arrays (with a total cost goal of less than $\$ 10 / \mathrm{kA}$ m of superconducting wire); (2) Cast insulators (with a cost goal of less than $\$ 0.01 / \mathrm{V}$ of average accelerating gradient); (3) Ferromagnetic materials (with a cost goal of $\$ 5-10 / \mathrm{kg}$ ), and (4) Pulsers (with a cost goal of less than $10^{-5} \$ / W$ for switches, and less than $\$ 20 / \mathrm{J}$ for stored energy). The final element of the Phase I program is development of an end-to-end simulation capability for both the IRE and full-scale drivers. This goal requires simulating a beam from the source, transporting it through the injector, accelerator, drift-compression section, final focus magnets, and target chamber, and then using the final distribution function of the beam at the focal spot as the input into the radiation-hydro code used to simulate the target dynamics. As currently envisioned, different codes would handle the accelerator and target chamber, with the results from the accelerator section serving as input into the chamber simulations. See Refs. $[4,5]$ for more detailed discussions of the simulation program.

\section{Example IRE design}

Early designs of the IRE have been based on analytic scaling laws $[6,7]$, which we describe below. The designs grew out of earlier work on pians for the so-called High Temperature Experiment (HTE) (Ref. [8]). These initial designs were intended for use as test-beds for simulations and to estimate final capabilities at the focal spot. It should be emphasized that these designs are starting points, and the discussions that follow are intended to give a general understanding of the scaling; detailed designs will result from more comprehensive systems studies. From the semianalytic designs, a program written in the Python interpreted language specifies detailed placements of system components that are required for simulations in the Particle-In-Cell (PIC) code WARP. A single slice of the beam can be simulated using the $2 D$ version of the code WARP2D, and emittance growth, halo formation, and sensitivities to accelerator errors can be studied. To simulate the full 3D dynamics of the beam, accelerating voltage waveforms must first be generated. At present the transverse envelope/ longitudinal fluid code CIRCE is used to generate these waveforms which are then fed to the 3D PIC code WARP3d (see Ref. [4]).

In an induction linac, several limits constrain the design. The phase advance per lattice period, $\sigma_{0}$, must be kept less than about $85^{\circ}$ to avoid envelope/lattice instabilities. For magnetic focusing, the phase advance is related approximately [9] to the magnetic field gradient $B^{\prime}$ by $\left(2\left[1-\cos \sigma_{0}\right]\right)^{1 / 2}=\eta B^{\prime} L^{2}(1-2 \eta / 3)^{1 / 2} /[B \rho]$, where $\eta$ is the quad occupancy, $L$ is the half-lattice period, and $[B \rho]$ is the ion rigidity. For electric quadrupolar focusing, in the expression for $\sigma_{0}, B^{\prime}$ is replaced by $E^{\prime} / v$, where $E^{\prime}$ is the transverse electric field gradient, and $v$ is the ion longitudinal velocity.

Space charge is limited by external focusing, described by the relation $K<\left(\sigma_{0} a / 2 L\right)^{2}$, where $K$ is the generalized perveance (proportional to line charge density $\lambda$ over ion voltage $V$ [i.e. ion energy over ion charge] for a non-relativistic beam), and a is the average beam radius.

A third limit is the velocity tilt, $\Delta v / v$, which for electrostatic quads must be less than about 0.3 to ensure that the tail radius and head $\sigma_{0}$ are not excessive and to minimize mismatches at the head and tail of beam. Here, $\Delta v$ is the difference between tail and head velocity at a fixed position along the accelerator. Since $\sigma_{0}$ is a more sensitive function of velocity for electric than for magnetic quads (scaling roughly as $1 / v^{2}$ for electric focusing and $1 / v$ for magnetic focusing), magnetic focusing allows a considerably larger $\Delta v / v$. Simulations will be carried out to establish the exact limit on $\Delta v / v$ for magnetic quadrupoles. The velocity tilt is given approximately by $\Delta v=(\mathrm{d} V / \mathrm{d} s)(l / v)\left(\frac{1}{2}-[V / I] \mathrm{d}\right.$ $l / \mathrm{d} V$, where $l$ is the bunch length of the flat-top of the beam and $l / v$ is the flat-top pulse duration. This expression is valid when the acceleration gradient length is much longer than the bunch 
length of the beam, as is true here. Note that for an accelerating beam, a velocity tilt is required to maintan a beam of constant bunch length $(\mathrm{d} l / \mathrm{d} V=0)$.

Another practical limit encountered by the linac designer is the number of volt-seconds per meter $(\mathrm{d} V / \mathrm{d} s) l / v$, where $\mathrm{d} V / \mathrm{d} s$ is the average accelerating gradient. This limit arises because for each core the volt-second product is given by, $V \Delta t=\eta_{\mathrm{cr}} \Delta \mathrm{BA}$ (from Faraday's law), where $\Delta t$ is the voltage pulse duration, $\Delta B$ is allowed magnetic flux swing before saturation sets in, $\eta_{\mathrm{cr}}$ is the radial packing fraction of the ferromagnetic material, and $A$ is the cross-sectional core area. $A$ is given by $\eta_{\mathrm{cl}}\left(R_{\mathrm{o}}-R_{\mathrm{i}}\right) L$, where $\eta_{\mathrm{cl}}$ is the longitudinal core occupancy, $R_{0}$ and $R_{\mathrm{i}}$ are the outer and inner radii of the induction cores. Thus $(\mathrm{d} V / \mathrm{d} s) l / v$ is proportional to $R_{\circ}-R_{i}$, which at present is taken to be less than approximately $1-1.5 \mathrm{~m}$. This translates into a limit on $(\mathrm{d} V / \mathrm{d} s) l / v$ $<\sim 1.3-2.0 \mathrm{Vs} / \mathrm{m}$ for $\Delta B=2.5 \mathrm{~T}, \quad \eta_{\mathrm{cr}}=0.8$, $\eta_{\mathrm{cl}}=0.8$, and $\Delta t /(l / v)=1.2$ to account for finite rise and fall times of current and voltage pulse.

A final practical limit in the accelerator is the average accelerating gradient $\mathrm{d} V / \mathrm{d} s$. Breakdown limits along insulators in the machine are currently assumed to limit the average acceleration to less than $\sim 1-2 \mathrm{MV} / \mathrm{m}$. Insulator research and systems studies are aimed at extending and more precisely defining this limit.

We have obtained a rough design for a possible IRE [10]. It is composed of 32 beamlines of singly charged potassium, accelerated to $200 \mathrm{MeV}$. The initial pulse duration is $6.69 \mu \mathrm{s}$, and the final pulse energy summed over all 32 beamlines is $30 \mathrm{~kJ}$. It is composed of three main sections.

(1) Electrostatic quadrupoles; constant bunch length.

(2) Magnetic quadrupoles with bunch compression; constant $\mathrm{Vs} / \mathrm{m}$.

(3) Magnetic quadrupoles; constant bunch length and maximum acceleration gradient. In addition, transitions between the sections are required. The scaling and values of parameters in this example (denoted the "reference IRE accelerator") are summarized in Table 1.

In the first section constant bunch length is assumed, $(\mathrm{d} l / \mathrm{d} V=0)$ and the line charge density $\lambda$ is also constant (at $0.27 \mu \mathrm{C} / \mathrm{m}$ ). The velocity tilt is held constant $(\Delta v / v=0.3)$ which leads to a scaling in that section such that the gradient $\mathrm{d} V / \mathrm{d} s \sim V$. The space charge limit implies $L \sim V^{1 / 2}$; and with a constant midpulse $\sigma_{0}$ (at $54^{\circ}$ ) and focusing gradient $E^{\prime}$, the occupancy $\eta$ is constant as well.

In the second section, the velocity tilt is such as to make acceleration and compression give equal contributions to the velocity tilt, yielding a bunch length $I$ that varies as $V^{-1 / 2}$. In this section, the $\mathrm{Vs} / \mathrm{m}$ is limiting and held constant so that $\mathrm{d} V / \mathrm{d} s \sim V$, as in Section 1. The space charge limit yields a half lattice period $L \sim V^{1 / 4}$. Constant $\sigma_{0}$ (at $70^{\circ}$ ) at maximum $B^{\prime}$ implies the quad occupancy $\eta$ is held constant (0.33).

Table 1

Scaling and summary of parameters for thereference IRE accelerator

\begin{tabular}{|c|c|c|c|c|c|c|}
\hline & $\begin{array}{l}\text { Bunch } \\
\text { length } \\
l(\mathrm{~m})\end{array}$ & $\begin{array}{l}\text { Half } \\
\text { lattice } \\
\text { period } L \\
\text { (m) }\end{array}$ & $\begin{array}{l}\text { Current } \\
\text { per beam } \\
I(\mathrm{~A})\end{array}$ & $\begin{array}{l}\text { Quad. } \\
\text { occup. } \\
\eta\end{array}$ & $\begin{array}{l}\text { Acceler. } \\
\text { gradient } \\
\mathrm{d} V / \mathrm{d} z \\
(\mathrm{kV} / \mathrm{m})\end{array}$ & $\begin{array}{l}\text { Focusing } \\
\text { gradient } \\
B^{\prime} \text { or } E^{\prime}\end{array}$ \\
\hline$V_{0}=1.6 \mathrm{MeV}$ & 18.8 & 0.21 & 0.77 & 0.65 & 55 & $1.4 \times 10^{8} \mathrm{~V} / \mathrm{m}^{2}$ \\
\hline Electric focusing & Constant & $\sim V^{1 / 2}$ & $\sim V^{1 / 2}$ & 0.65 & $\sim V$ & $1.4 \times 10^{8} \mathrm{~V} / \mathrm{m}^{2}$ \\
\hline$V_{1}=9.4 \mathrm{MeV}$ & 18.8 & 0.5 & 1.87 & Jump & 323 & Jump \\
\hline $\begin{array}{l}\text { Magnetic focusing } \\
\text { (compression) }\end{array}$ & $\sim V^{-1 / 2}$ & $\sim V^{1 / 4}$ & $\sim V$ & 0.33 & $\sim V$ & $37 \mathrm{~T} / \mathrm{m}$ \\
\hline$V_{2}=29.8 \mathrm{MeV}$ & 10.6 & 0.67 & 5.9 & 0.33 & 1000 & $37 \mathrm{~T} / \mathrm{m}$ \\
\hline $\begin{array}{l}\text { Magnetic focusing (const. } \\
\text { bunch length) }\end{array}$ & Constant & $\sim V^{1 / 2}$ & $\sim V^{1 / 2}$ & $\sim V^{1 / 2}$ & Constant & Slow variation \\
\hline$V_{3}=200 \mathrm{MeV}$ & 10.6 & 1.73 & 15.3 & 0.127 & 1000 & $34.1 \mathrm{~T} / \mathrm{m}$ \\
\hline
\end{tabular}


When the maximum gradient is reached, a third section is started in which the gradient is frozen at the maximum value $(\mathrm{d} V / \mathrm{d} s=1000 \mathrm{kV} / \mathrm{m})$. To avoid excessively large electric fields in the "ears", we also maintain a constant bunch length in this section. Constant bunch length, together with constant beam radius, yields a half lattice period scaling $L \sim V^{1 / 2}$ and a constant velocity tilt $\Delta v / v$. In this, the longest of the three sections it is convenient to also freeze magnet length, assuming a cost benefit will accrue from such standardization. The scaling on the occupancy then follows the relation $\eta \sim V^{-1 / 2}$; and the assumption of constant $\sigma_{0}$ requires the focusing gradient $B^{\prime} \sim 1 /(1-2 \eta / 3)^{1 / 2}$.

Towards the end of the third accelerator section, as the beam is approaching $200 \mathrm{MeV}$, a velocity tilt is placed upon the beam to longitudinally compress it in a drift compression section after the accelerator. Although we have not yet done detailed simulations of the IRE beam in the drift compression section, we have made analytic estimates to characterize the final beam parameters that could be achieved on target. The final peak line charge density $\lambda_{a}$ at the output of the accelerator would be $0.73 \mu \mathrm{C} / \mathrm{m}$. (This is 1.5 times larger than the peak flat-top line charge density, to account for a parabolic density profile we assume in the analytic estimates.) The associated peak generalized perveance $K_{\mathrm{a}}$ is then $3.3 \times 10^{-5}$. We choose three different values of final pulse duration $\Delta t_{\mathrm{f}}(5,10$, and $20 \mathrm{~ns})$ to estimate the final parameters. These are summarized in Table 2 , below. Note that in the table that subscript "f" indicates parameters evaluated at the final focal spot, and subscript "a" indicates that it is to be evaluated at the end of the accelerator (prior to drift compression).

In Table 2, the longitudinal space charge factor $g \equiv-4 \pi \varepsilon_{0} \mathrm{E}_{\mathrm{z}} /\left(\partial \lambda / \partial z^{\prime}\right) \cong 2 \ln \left(r_{\mathrm{p}} / a\right)$ was assumed to be $1.3, k_{\mathrm{B}}$ is the Maxwell-Boltzmann constant, $\sigma$ is the Stefan-Boltzmann constant, $E$ is the total pulse energy, and $z^{\prime}$ is the longitudinal distance in the comoving beam frame. The compression ratio $C \equiv l_{\mathrm{a}} / l_{\mathrm{f}}$ is the ratio of the bunch length at the end of the accelerator to that at the target.

The final two columns in the table display estimates of the maximum spot temperature for the three different pulse durations. The quantity $k_{\mathrm{B}} T_{\max }$ is the temperature (in $\mathrm{eV}$ ) found from equating the energy flux $F$ onto the target with the radiative flux from the target $\sigma T^{4}$. A more detailed model in Ref. [11] takes into consideration the energy required to heat the target. In this model, the temperature of the target evolves according to $R \mathrm{~d} \varepsilon / \mathrm{d} t=F-\sigma T^{4}$, where $R$ is the ion range (approximately $0.012 \mathrm{~g} / \mathrm{cm}^{2}$ ) and $\varepsilon$ is the specific energy of the target material. In Ref. [11], using the Raiser-Zeldovich approximation to calculate the ionization state of the matter, it was found that $\varepsilon$, in aluminum at relevant temperatures, was approximately given by $\varepsilon=7750 \mathrm{~J} / \mathrm{g}$ $\left(k_{\mathrm{B}} T / 1 \mathrm{eV}\right)^{1.5}\left(\rho / 1 \mathrm{~g} / \mathrm{cm}^{3}\right)^{0.12}$, where $\rho$ is the mass density of the target material. For the table we

Table 2

Final beam and drift compression parameters for reference accelerator as a function of $\Delta t_{\mathrm{f}}$

\begin{tabular}{|c|c|c|c|c|c|c|c|c|}
\hline \multirow{3}{*}{$\begin{array}{l}\text { Final } \\
\text { pulse } \\
\text { duration } \\
\Delta t_{f} \text { (ns) }\end{array}$} & \multirow{3}{*}{$\begin{array}{l}\text { Compression } \\
\text { ratio } C \\
l_{\mathrm{a}} / l_{\mathrm{r}}\end{array}$} & \multirow{3}{*}{$\begin{array}{l}\begin{array}{l}\text { Velocity tilt } \\
(\Delta v / v)_{\mathrm{a}}\end{array} \\
{\left[8 K_{\mathrm{a} g} \times\right.} \\
(C-1)]^{1 / 2}\end{array}$} & \multirow{3}{*}{$\begin{array}{l}\begin{array}{l}\text { Drift } \\
\text { length } \\
d_{\text {drift }}(\mathrm{m})\end{array} \\
\begin{array}{l}l_{\mathrm{a}}(1-1 / C) / \\
\Delta v / v\end{array}\end{array}$} & \multirow{3}{*}{$\begin{array}{l}\text { Energy flux } \\
F\left(\mathrm{~W} / \mathrm{cm}^{2}\right) \\
E /\left(\pi r_{\mathrm{f}}^{2} \Delta t_{\mathrm{f}}\right)\end{array}$} & \multicolumn{2}{|c|}{$k_{\mathrm{B}} T_{\max }(\mathrm{eV})$} & \multirow{2}{*}{\multicolumn{2}{|c|}{$\begin{array}{l}k_{\mathrm{B}} T_{\text {model }}(\mathrm{eV}) \\
\text { (cf. Ref. [11] }\end{array}$}} \\
\hline & & & & & \multicolumn{2}{|c|}{$k_{\mathrm{B}}(F / \sigma)^{1 / 4}$} & & \\
\hline & & & & & $\begin{array}{l}r_{\mathrm{f}}=5 \\
(\mathrm{~mm})\end{array}$ & $\begin{array}{l}r_{f}=2.5 \\
(\mathrm{~mm})\end{array}$ & $\begin{array}{l}r_{\mathrm{f}}=5 \\
(\mathrm{~mm})\end{array}$ & $\begin{array}{l}r_{\mathrm{f}}=2.5 \\
(\mathrm{~mm})\end{array}$ \\
\hline 5 & 67 & 0.150 & 71.7 & $7.6 \div 10^{12}$ & 93 & 131 & 43 & 101 \\
\hline 10 & 33.5 & 0.106 & 101 & $3.8 \times 10^{12}$ & 78 & 110 & 43 & 95 \\
\hline 20 & 16.8 & 0.074 & 140 & $1.9 \times 10^{12}$ & 66 & 92 & 42 & 87 \\
\hline
\end{tabular}


assume a felted aluminum target at a density of $0.1 \mathrm{~g} / \mathrm{cm}^{3}$. It can be seen that for a $5 \mathrm{~mm}$ spot, temperatures of order $43 \mathrm{eV}$ can be achieved, whereas one would need to achieve a $2.5 \mathrm{~mm}$ spot radius for $100 \mathrm{eV}$ temperatures. These calculated temperatures could be lower if hydrodynamic motion cools the spot or higher if radiation is trapped by means of opaque foils, or geometries (such as cylindrical hohlraums proposed for the ITEP TWAC experiments (Ref. [12])) which focus the radiation. More detailed calculations are in progress.

Because the final ion velocity at the end of the accelerator is likely to be less than in a driver, the generalized perveance for the IRE is likely to be larger than it is in a driver. Since the generalized perveance indicates the importance of space charge relative to the kinetic energy of beam particles, it plays an important role in determining the focusability. To meet the $5 \mathrm{~mm}$ or smaller spot radius goal, for this example accelerator the beam must be neutralized.

To estimate the degree of neutralization required we consider the envelope equation when only space charge is included: $\mathrm{d}^{2} a / \mathrm{d} z^{2}=(1-f) K_{\mathrm{a}} C / a$. Here $a$ is the beam radius, and $f$ is the fraction of the beam space charge that has been neutralized. At the exit of the final magnet, where the beam radius has value $a_{0}$, one integration of the envelope equation implies $f \cong 1-\theta^{2} /\left(2 K_{\mathrm{a}} C \ln \left(a_{\mathrm{o}} / r_{\mathrm{f}}\right)\right)$, where $\theta$ is the focusing half angle $\theta \cong a_{0} / d$, and $d$ is the focal distance, which we assume to be $2 \mathrm{~m}$ for the IRE. Neutralization fractions between $89-97 \%$ (for final pulse duration $\Delta t_{\mathrm{f}}$ between 20 and $5 \mathrm{~ns}$, respectively) are required for convergence angles less than or equal to $15 \mathrm{mrad}$. The neutralization fractions may be limited to values of order 1 $\alpha q m_{\mathrm{e}} /\left(C K_{\mathrm{a}} A m_{\mathrm{amu}}\right)$ [13] for electrons that are picked up axially, where $\alpha$ is between 1 and $4, m_{e}$ and $m_{\text {amu }}$ are the electron mass and atomic mass unit, respectively, and $A$ is the ion mass in amu. For the worst case, $\alpha=4$, this would span the range $90-97 \%$, respectively. The two constraints on $f$ can be written $(A / q)>2\left(m_{\mathrm{e}} / A m_{\text {amu }}\right) \alpha(\ln [\theta d)$ $\left.\left.r_{\mathrm{f}}\right]\right) / \theta^{2} \cong 35$ for $\alpha=4$. The reference IRE parameters are thus consistent with this inequality, but detailed simulations of the neutralization process will, of course, be carried out for a variety of neutralization methods. Contributions to the spot size from the emittance $\delta r_{\varepsilon} \cong \varepsilon_{\mathrm{a}} / \beta \theta$ and from chromatic aberrations (assuming uncorrected optics) $\delta r_{\delta p / p} \cong 6 d \theta \delta p / p$ must also be taken into consideration. A normalized emittance $\varepsilon_{\mathrm{n}}$ less than 3-5 $\mathrm{mm} \mathrm{mrad}$ and momentum spread $\delta p / p$ less than $1 \%$ would satisfy the spot radius requirement of less than or equal to $5 \mathrm{~mm}$.

The upper end of the compression ratios proposed for this example IRE would exceed those that are required for a driver. One issue arising from a large compression ratio is the voltage variation allowed at each accelerating module and at the injector [14]. Since errors in the voltage waveforms at the ith module, $\delta V_{i}$ will get translated into longitudinal momentum variations $\delta p_{\mathrm{I}}=\left(p_{i} / 2 V_{i}\right) \delta V_{i}$, a specification can be set on the allowed variation in the voitage waveforms. As the pulse length shortens, longitudinal phase space is conserved $\delta p_{i} l_{i}=\delta p_{\mathrm{a}} l_{\mathrm{a}}$, so the momentum variations increase. The larger the compression ratio, the larger the growth of the perturbation. At each module, a number of pulsers $N_{p}=V_{i} / V_{\text {pulser }}$ add together, so that fluctuations add statistically, with $\delta V_{i}=\left(V_{i} / V_{\text {pulser }}\right)^{1 / 2} \delta V_{\text {pulser. }}$. Here $V_{i}$ is the voltage increment at the $i$ th module, and $V_{\text {puiser }}$ is the pulser voltage, which we take to be $10 \mathrm{kV}$. Also, weighing each perturbation by the ratio of the bunchlength at each module $l_{i}$, to its value at the end of the accelerator $l_{a}$, we estimate the momentum variation at the end of the accelerator $\delta p_{\mathrm{a}} / p_{\mathrm{a}}:\left(\delta p_{\mathrm{a}} / p_{\mathrm{a}}\right)^{2}=$ $\Sigma(1 / 4)\left(V_{i} V_{\text {pusser }} / V_{\mathrm{a}}^{2}\right)\left(\delta V_{\text {pulser }} / V_{\text {puser }}\right)^{2}\left(\Delta t_{i} / \Delta t_{\mathrm{a}}\right)^{2}+$ $(1 / 4)\left(\delta V_{\text {injector }} / V_{\text {injector }}\right)^{2}\left(\Delta t_{\text {injector }} / \Delta t_{\mathrm{a}}\right)^{2}$. Here, the sum in the first term is over all acceleration modules, and the second term arises from injector voltage variations, weighted appropriately. Further compression in the drift section increases the momentum spread at the target $\delta p_{\mathrm{t}} / p_{\mathrm{t}}$, such that $\delta p_{\mathrm{t}} / p_{\mathrm{t}}=C \delta p_{\mathrm{a}} / p_{\mathrm{a}}$. For a $\delta p_{\mathrm{t}} / p_{\mathrm{t}}=0.01$, and equal contributions from pulser errors and injector voltage errors, this limits $\delta V_{\text {pulser }} / V_{\text {pulser }}=$ $0.01-0.03$, and $\delta V_{\text {injector }} / V_{\text {injector }}=0.001-0.005$, for $C=67-17$, respectiveiy.

Ajother concern is the robustness of the spot radius under variations in the onset of neutralization in the final chamber. The envelope 
equation in the final chamber is: $\mathrm{d}^{2} a / \mathrm{d} z^{2}=$ $(1-f) K_{\mathrm{a}} C / a+\varepsilon^{2} / a^{3}$. If the neutralization point (location where $1-f$ approaches zero) is delayed by a distance $\delta l$, the spot radius will increase by an amount $\delta r \cong f K_{a} C d\left(\delta l / a_{0}\right)=4$ to $1 \mathrm{~mm}$, for $C$ $=67$ to 17 respectively, and assuming that $\delta l / a_{0}=1$. This uncertainty is largest for large $C K_{\mathrm{a}}$ and would be of greatest concern if $\delta l$ were to be variable from pulse to pulse.

Another requirement in the transport of high line charge density beams is that the radial electric field of the beam does not cause breakdown at the beam pipe wall. This constraint is easily quantified from an estimate of the radial electric field $E$ $=\lambda /\left(2 \pi \varepsilon_{0} r_{\mathrm{p}}\right)=16$ to $4 \mathrm{MV} / \mathrm{m}$ for $C=67$ to 17 , respectively, and where the pipe radius $r_{\mathrm{p}}=5 \mathrm{~cm}$. These values should be achievable, particularly over the duration of the short 5-20 ns pulse.

Finally, third order geometric aberrations are a concern for beams of convergence angles which may be needed for high line charge density. These are currently under study using the WARP code, and may also place limits on IRE generalized perveance at the final focus.

Computer simulation of beam dynamics plays an essential role in the analysis and design of the IRE. Areas of investigation are numerous. They include aberrations, longitudinal/transverse coupling, interactions through module impedance, mismatches from acceleration and errors, multiple beam interactions, neutralization and stripping in chamber, to name a few. See Refs. [4,5] for further details. Validation of the computational toois on near-term experiments leads to confidence in future designs.

The reference IRE parameters describe an accelerator which would address nearly every non-target issue needed to proceed to the ETF. The high generalized perveance of the beams would provide a rigorous test of individual beam acceleration, compression, and focusability. The final design will ultimately reflect all beam manipulations that would be found in a driver (e.g. if systems studies suggest that beam merging will be necessary in a driver, then an IRE will also be required to demonstrate beam merging). A number of chamber transport methods (including ballistic neutralized transport, channel transport, and self-pinch transport) will be tested. The final extrapolation to the higher ion energy in the ETF will rely on atomic cross-sections obtained from high-energy accelerators such as those at GSI, together with extrapolation of lower energy data from the IRE, theory and simulations. Technology issues of lifetime and maintainability will be demonstrated on the IRE and when necessary on small off-line component tests. Chamber technology issues may, to a large extent, be tested in separate facilities, although issues of driver/chamber interface (such as allowable stand-off distance between beam and shielding material) will be addressable in the IRE [15].

\section{Systems studies}

Computer aided optimization is also beginning to play an important role in the design of the machine. The systems code IBEAM (cf. [16,17]) is being developed and used to find optimal designs. Fig. 1 illustrates the variation in cost (relative to the case with the nominal parameters listed above) for an IBEAM-generated design with parameters essentially the same as the reference accelerator, with a 5 ns pulse duration on target. In the figure, $M$ is the design parameter divided by the nominal

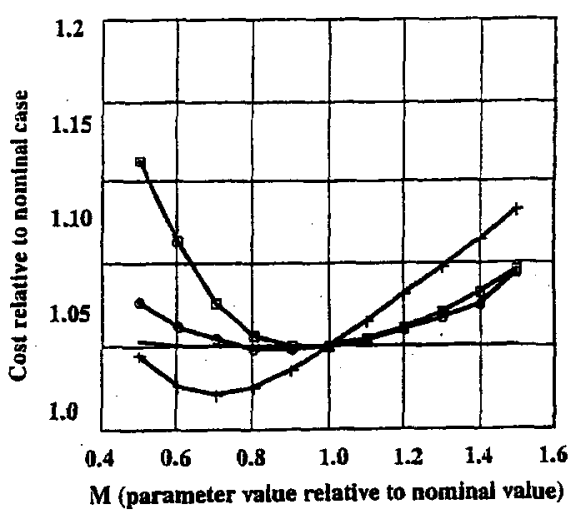

Fig. 1. Relative cost vs. selected design parameters (relative to nominal). The nominal parameters are quadrupole field at winding (circles, nominal value $4 \mathrm{~T}$ ), initial pulse duration (squares, nominal value $6.69 \mu \mathrm{s}$ ), number of beams (crosses, nominal value 32 ) and transition energy from electric to magnetic (no symbol, nominal value $9.43 \mathrm{MeV}$ ). 
design parameter for each curve. $M=1$ is for the nominal design. The parameters varied were initial pulse duration (squares), quadrupole field (circles), number of beams (crosses), and transition energy from electric to magnetic quads (no symbol).

Notice that only the number of beams appears not. to be optimum in the design. However, the nominal number of beams is consistent with the spot size and the assumed degree of neutralization (98\%) which may not be the case with fewer beams. Also note that for the parameters shown, variations in cost are small (less than 12\%) indicating broad optima are likely in the final design.

\section{Summary and conclusions}

A multi-pronged Phase 1 research effort is laying the groundwork for the Integrated Research Experiment. Experiment, technology development, theory, simulation, and systems studies are all playing major roles in this Phase I research. The key research areas are: (1) Source and injector (for investigation of a high brightness, multiple beam, low cost injector); (2) High current transport (to examine effects at full driver-scale line charge density, including the maximization of the beam filling-factor and control of electrons); (3) Enabling technology development (low cost and high performance magnetic core material, superconducting magnetic quadrupole arrays, insulators, and pulsers); (4) Beam simulations and theory (for investigations of beam matching, specification of accelerator errors, studies of emittance growth, halo, and bunch compression, in the accelerator, and neutralization methods, stripping effects, spot size minimization in the chamber); and (5) Systems optimization (minimization of cost and maximization of pulse energy and beam intensity).

We have begun the process of designing, simulating, and optimizing the next major heavy-ion induction accelerator, the IRE. This accelerator facility will, in turn, help provide the basis to proceed to the next step in the development of IFE as an attractive source of fusion energy.

\section{References}

[1] R.O. Bangerter, The heavy ion fusion program in the USA, Nucl. Instr. and Meth. A 464 (2001) 17, these proceedings.

[2] J.W. Kwan, D.N. Beck, F.M. Bieniosek, A. Faltens, E. Henestroza, L. Ahle, D.P. Grote, E. Halaxa, V. Karpenko, T.C. Sangster, W.B. Herrmannsfeidt, Ion sources and injectors for induction linacs, Nucl. Instr. and Meth. A 464 (2001) 379 , these proceedings.

[3] P.A. Seidl, Experiments in the USA Virtual National Laboratory, Nucl. Instr. and Meth. A 464 (2001) 369, these proceedings.

[4] A. Friedman, Beam simulations for IRE and driver-status and strategy, Nucl. Instr. and Meth. A 464 (2001) 653, these proceedings.

[5] D.P. Grote, A. Friedman, G. Craig, I. Haber, W.M. Sharp, Progress toward source-to-target simulation, Nucl. Instr. and Meth. A 464 (2001) 563, these proceedings.

[6] C.H. Kim, L. Smith, Part. Accel. 18 (1985) 101.

[7] E.P. Lee, Heavy ion driven LMF design concept UC-421, LBL-31248 revised, HIFAN721, July 1995.

[8] A. Faitens, HTE update, LBNL HIFAR 468, 1996.

[9] E.P. Lee, T.J. Fessenden, L.J. Laslett, IEEE Trans. Nucl. Sci. NS-26 (1985) 2489.

[10] E.P. Lee, New IRE, private communication July 16, 1998; LBNL HIFAN note, in preparation.

[11] E.P. Lee, J.W-K. Mark, Spot heating calculation for a heavy ion driven high temperature experiment, LBL16890, HIFAN-226, April 1984.

[12] B.Y. Sharkoy, N.N. Alexeev, M.D. Churazov, A.A. Golubev, P.R. Zenkovich, D.G. Koshikarev, Heavy ion fusion energy program in Russia, Nucl. Instr. and Meth. A 464 (2001) $i$, these proceedings.

[13] C. Olson, Chamber transport, Nucl. Instr. and Meth. A 464 (2001) 118, these proceedings.

[14] A. Warwick, E.P. Lee, Acceleration errors in MBE-4 and in a driver, HIFAR 214, 1988.

[15] P.F. Peterson, HIF liquid hydraulics scaling and pocket design, Nucl. Instr. and Meth. A 464 (2001) 159, these proceedings.

[16] W.R. Meier, R.O. Bangerter, A. Faltens, Nucl. Instr. and Meth. Phys. Res. A 415 (1998) 249.

[17] W.R. Meier, J.J. Barnard, R.O. Bangerter, Evaluating heavy ion driver design options using an integrated systems model, Nucl. Instr. and Meth. A 464 (2001) 433, these proceedings. 


\title{
OVERVIEW OF THE SCIENTIFIC OBJECTIVES OF THE HIGH CURRENT EXPERIMENT FOR HEAVY-ION FUSION*
}

\author{
P. Seidl ${ }^{\dagger}$, R. Bangerter, C. Celata, A. Faltens, V. Karpenko, E. Lee, LBNL, Berkeley CA 94720, I. Haber, NRL, \\ Washington, D.C. 20375, S. Lund, A. Molvik, LLNL, Livermore, CA 94550
}

\begin{abstract}
The High Current Experiment (HCX) is being built to explore heavy-ion beam transport at a scale appropriate to the low-energy end of a driver for fusion energy production. The primary mission of this experiment is to investigate aperture fill factors acceptable for the transport of space-charge dominated heavy-ion beams at high space-charge intensity (line-charge density $-0.2 \mu \mathrm{C} / \mathrm{m}$ ) over long pulse durations $(3-10 \mu \mathrm{sec})$. A single beam transport channel will be used to evaluate scientific and technological issues resulting from the transport of an intense beam subject to applied field nonlinearities, envelope mismatch, misalignment-induced centroid excursions, imperfect vacuum, halo, background gas and electron effects resulting from lost beam ions. Emphasis will be on the influence of these effects on beam control and limiting degradations in beam quality (emittance growth). Electrostatic (Phase I) and magnetic (Phase II) quadrupole focusing lattices have been designed and future phases of the experiment may involve acceleration and/or pulse compression. The Phase I lattice is presently under construction[1] and simulations to better predict machine performance are being carried out [2]. Here we overview: the scientific objectives of the overall project, processes that will be explored, and transport lattices developed.
\end{abstract}

\section{INTRODUCTION}

The Heavy-Ion-Fusion (HIF) Program's objective is to provide a comprehensive scientific knowledge base for inertial fusion energy (IFE) driven by high-brightness heavy-ion beams. The HCX will explore beam transport limitations at a scale characteristic of the low-energy end of a multi-beam induction linac driver, using a single beam to save on cost. Advances in the understanding of the physics of intense ion beam transport and acceleration of a single beam will enable better determination of the optimal beam aperture filling factor and pulse duration. Filling the apertures as much as possible is very important in multi-beam induction linacs in order to minimize the transverse cross-sectional area of multi-beam focusing arrays and, in turn, the amount of induction core material needed for economical acceleration. Thus, it is important to maximize the beam current density over the crosssection of the array. The optimum will be linked to the choice of beam parameters and focusing technology. Small beam-edge to aperture clearances at high intensities

* This work supported by the Office of Energy Research, U.S. Department of Energy, the Lawrence Berkeley and Livermore Labs under contract number DE-AC03-76SF00098 and W-7405-Eng-48. †PASeidi@lbl.gov make it crucial to understand how processes influence practical transport limits. These limits may be different in magnetic and electric focusing sections, and most costoptimal driver concepts employ both electric (low energy) and magnetic (higher energy) focusing sections.

The single-beam HCX will be the first heavy-ion electric and magnetic quadrupole transport experiment with simultaneously: high line charge density $(\lambda \sim 0.2$ $\mu \mathrm{C} / \mathrm{m})$, long pulse duration $(3<\tau<10 \mu \mathrm{s})$, and high injection energy $\left(1.6<\mathrm{E}<2.0 \mathrm{MeV}, \mathrm{K}^{+}\right.$, coasting beam). These parameters, for a driver, are believed to lie in the rather broad ranges: $0.1<\lambda<1.0 \mu \mathrm{C} / \mathrm{m}, 5<\tau<50 \mu \mathrm{s}$, and $1<\mathrm{E}<4 \mathrm{MeV}$ at injection. Previous scaled heavy-ion transport experiments had much less space-charge intensity $(\lambda<0.04 \mu \mathrm{C} / \mathrm{m})$ and were not capable of resolving scale-dependant issues in transport. A goal of the HCX is to identify and understand processes that constrain choices of machine aperture needed for high confidence, but attractive, driver designs.

\section{SCIENTIFIC OBJECTIVES}

Processes listed below can limit the transportable beam current. Moreover, the deleterious effects noted can be enhanced at the high fill factors desirable for HIF. The objective of the HCX is to obtain data on how these processes limit transportable beam current.

(1) Nonlinear fields: The transverse phase space of the beam can be diluted (emittance growth) due to nonlinear fields resulting from imperfections in the applied focusing and self-field effects such as image charges and nonlinear distribution of space-charge.

(2) Alignment errors: Misalignments cause oscillations in the beam centroid with a period characterized by $\sigma_{0}$, the undepressed particle phase advance. The oscillations bring the beam-edge closer to the physical aperture, resulting in larger nonlinear forces due to applied field errors and image charges. The frequency of needed steering corrections for tolerable centroid errors is important.

(3) Distribution errors: Distribution distortions and mismatches in phase-space can launch deleterious collective oscillations. Mismatches of the distribution to focusing structures lead to beam envelope oscillations that can pump large amplitude beam halo. Higher order distortions such as those resulting from space-charge nonuniformities may launch spectrums of collective waves that complicate beam control and can lead to emittance growth. These envelope and collective modes have rapid frequency oscillations that scale with the ion plasma frequency. Broad spectrums of modes can rapidly phase mix and undergo collective relaxation with some 
emittance growth, whereas lower order modes (narrow spectrum) can persist and complicate beam control.

(4) Lost particles: Beam interactions with background gas, charge exchange, and large amplitude beam halo can result in particles lost to the machine aperture. Over a long pulse, this can result in cascades of desorbed gas being released from surfaces and production of secondary electrons that will interact with the beam pulse. At high energy, lost beam ions can also activate accelerator structures. The yield of desorbed atoms is expected to be large and has considerable impact on the needed aperture, but little relevant data exists to help fix needed beam edge to aperture clearances for characteristic HIF pulse durations and vacuum systems. Secondary electrons are also an issue of strong concern in magnetic focusing systems. Such systems lack the strong sweeping fields associated with electric focusing, increasing chances that electrons will be trapped in the multi-kV potential well of the ion-beam thereby leading to streaming instabilities, partial neutralization, and loss in beam control.

(5) Longitudinal physics: Matching of the beam head and maintaining beam control over the pulse in the presence of acceleration and velocity tilt for compression introduces various constraints. Beam-beam interactions in acceleration gaps (electric and magnetic) and focusing arrays (magnetic) may also complicate beam control.

\section{EXPERIMENTAL APPROACH}

Phase I will primarily test the physics of electrostatic quadrupole (ESQ) transport and Phase II will test magnetic quadrupole transport with one coasting, driverscale beam. To address scientific transport issues early, with limited funding, near-term plans do not include beam acceleration and compression or mutibeam arrays to study multi-beam control and beam-beam interactions. However, later stages will include at least one induction cell for the study of bunch end control and longitudinal space-charge waves. The Phase II lattice is being designed to allow retrofits for beam acceleration and compression. Some multibeam elements may be incorporated to allow mock-up of multibeam effects.

Phase I

The experimental hardware includes a $0.6 \mathrm{~A}, 1.8 \mathrm{MeV}$ $\mathrm{K}^{+}$injector and matching section $[3,4]$, followed by a 20 period FODO transport lattice of $40 \mathrm{ESQs}$ (4 tanks, each with 10 ESQs), and then four pulsed magnetic quadrupoles. The ESQ lattice has a half-period of $L=21.76 \mathrm{~cm}$, a clear bore radius of $r_{p}=2.3 \mathrm{~cm}$, and a hardedge equivalent quadrupole occupancy of $\eta \approx 0.7$ [1]. The ESQs will be aligned to within tolerances of $\pm 75 \mu \mathrm{m}$ of center, and the ESQ electrodes will be biased to $V \approx$ $\pm 55 \mathrm{kV}$ at the maximum allowable focusing strength (i.e., the envelope stability limit corresponding to $\sigma_{\mathrm{o}}=90^{\circ}$ ). The HCX ESQs are derived from a simple design of cylindrical rods mounted to end-plates, with the radii of the rods chosen to be $(8 / 7) r_{p}$ to eliminate lowest-order systematic multipole errors (dodecapole component) in $2 D$ transverse geometry. The clear bore radius $r_{p}$ was set from estimates of needed beam-edge to aperture clearances including centroid errors and mismatch. Electrode gaps were set from high-voltage breakdown tests[5]. A prototype ESQ was tested to $\mathrm{V}= \pm 120 \mathrm{kV}$ (> $2 \mathrm{x}$ operating) without breakdown. A system base pressure of less than $10^{-7}$ Torr is expected.

Considerable flexibility has been designed into the lattice to allow the aperture to be probed with the beam, and effects measured. In each tank of 10 ESQs, the first ESQ will be removable to allow space for horizontal and vertical plane slit-scanners for measuring the beam phasespace in each transverse plane. This ESQ may also be rotated (up to $\pm 4^{\circ}$ ) about the reference beam centroid to introduce variable skew coupling for the study of rotational alignment tolerances. At the end of each tank, the voltages on the final four ESQs may be varied independently to adjust beam envelope excursions for matching, or purposely mismatching the rms envelope. The last two ESQs can also be displaced along the horizontal and vertical directions (over $\pm 1.2 \mathrm{~cm}$ with \pm 12 $\mu \mathrm{m}$ resolution) to allow the beam centroid to be steered in offset and angle. There will also be slit-scanner diagnostics in a tank at the end of the machine and the matching section before the first tank also has provisions for beam steering. Thus the beam centroid and envelope may be controlled and diagnostics performed near all tank to tank transitions. Initial experiments with a first tank of 10 quadrupoles are scheduled to start late in 2001.

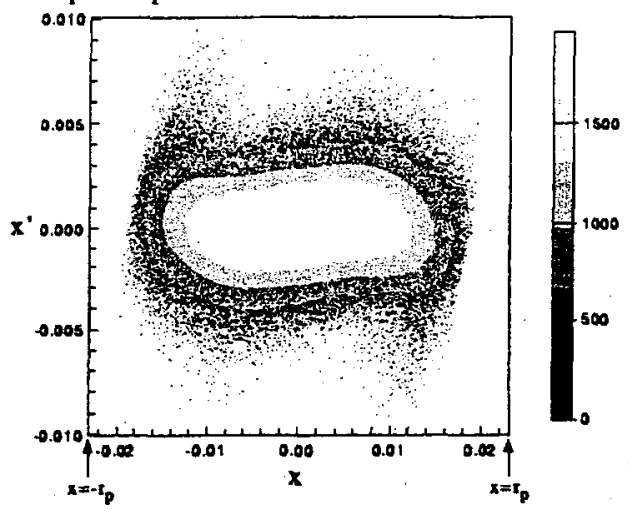

Figure 1: Transverse $x-x^{\prime}$ phase space from a 2D PIC simulation of an initially uniform (semi-Gaussian) beam transported through 5 periods of the Phase I lattice at reduced focusing strength $\left(\sigma_{0}=55^{\circ}\right)$ to fill the aperture.

An experimental program has been developed to explore emittance growth and beam control with variations in the aperture fill factor. The maximum transverse matched beam-edge excursion with zero centroid error is about $62 \%$ of the aperture at a nominal focusing strength corresponding to $\sigma_{0}=80^{\circ}$. Beam excursions in the aperture will be increased and phasespace distortions measured (emittance growth) by: lowering the focusing gradient (i.e., decreasing $\sigma_{0}$ ), deliberately inducing envelope mismatch with controlled 
amplitude and phase, and by steering the beam centroid away from the nominally aligned condition. Contingent on early results, the aperture on subsequent tanks may also be changed. To probe sensitivities, the 40 quadrupole lattice will be long enough to allow -5 undepressed and -1.2 depressed characteristic single-particle oscillations, and $\sim 6.9$ collective plasma periods of space-charge mode evolution. A typical distribution distortion obtained for a large fill factor with an aligned beam (zero centroid error) is shown in Fig. 1.

The beam for the HCX will be produced from a retrofit ESQ injector and an ESQ matching section that were originally constructed at LBNL to deliver a $2 \mathrm{MeV}$, $800 \mathrm{~mA} \mathrm{~K} \mathrm{~K}^{+}$beam for the Elise project[3]. The optics of the injector diode have been improved, the pulse length extended[5], and stable operation at $1.8 \mathrm{MeV}$ and $0.6 \mathrm{~A}$ is expected. Simulations and experiment found that various processes could result in complex distribution distortions at the_exit of the injector. The retrofits have reduced these distortions, and the beam has been diagnosed extensively at the exit of the injector so that limitations imposed can be understood. If necessary, further retrofits will be carried out during the Phase I experiments.

Four pulsed magnetic quadrupoles designed for a prototype 21-beam array have been built and tested for $>80,000$ pulses [6] and will be adapted for use at the end of the Phase I lattice. The quadrupoles have an elliptical clear bore aperture with radii 3.0 and $5.0 \mathrm{~cm}$, an effective axial field length of $31 \mathrm{~cm}$, and a gradient of $40 \mathrm{~T} / \mathrm{m}$. This short lattice will provide an early, inexpensive opportunity to develop diagnostics sensitive to electrons that may become entrained in the self-field potential well of the ion beam in the absence of sweeping fields associated with ESQ focusing.

\section{Diagnostics}

Various pulse-resolved diagnostics will be available. Double-slit intercepting diagnostics similar to those employed on the ESQ injector will be employed to measure the transverse phase space ( $x-y, x-x^{\prime}$ and $\left.y-y^{\prime}\right)$ of the jon beam along the horizontal $(x)$ and vertical (y) planes. This technique relies on the demonstrated shot-toshot repeatability of such systems. The total ion linecharge density $\lambda_{\text {ion }}(t)$ (or current) will be accurately measured (1-2\%) with a beam-enclosing Faraday cup. A nearby Rogowski loop will measure net line-charge $\lambda_{\text {net }}(t)$ (or current). Together, these will give information on beam loss and the degree of partial beam neutralization due to secondary electrons. A valve will be present to puff gas into the vacuum system and vary the background pressure and beam-gas interactions. Halo-induced desorption will be detected with ionization gauges. A gated beam imager is being evaluated for rapid, one-shot phase-space measurements complimentary to those of the slit scanners. This system works by imaging pinholes and can provide full cross-plane correlation data on the beam distribution.
The experiment will also be employed as a test bed for new diagnostics, particularly non-intercepting and electron diagnostics. Azimuthally segmented capacitive probes will be employed to measure $\lambda_{\text {mes }}(t)$ and ratios of beam envelope radii. These probes can be installed inside the bores of magnetic quadrupoles, where electrons may be trapped in orbits around the quadrupole field lines. Lowfrequency interferometry and compact energy analyzers are being evaluated to measure secondary electron effects.

\section{Phase II}

Preliminary plans call for the lattice to be extended by the addition of 50-100 magnetic quadrupoles to allow sensitive probing of magnetic transport limits, which are less understood than electric limits. A syncopated FODO lattice is envisioned with periodicity $2 \mathrm{~L}=45 \mathrm{~cm}$, clear bore radius $r_{p}=3 \mathrm{~cm}$, and a quadrupole occupancy of $\eta=0.45$. Two independent superconducting magnet designs have been successfully prototyped[7]. One of these prototypes achieved, without quench training, an integrated gradient of $8.5 \mathrm{~T}$ needed for matched beam focusing. Selfconsistent beam simulations indicated that end compensations may be employed to achieve high average field quality with simple magnet designs, thereby allowing considerable design freedom. Cryostat and lattice period designs have up to $8 \mathrm{~cm}$ free, warm axial space within the lattice period for diagnostics, pumping, acceleration gaps. Although plans call for initial coasting beam experiments, hardware is being designed to allow a later upgrade with acceleration gaps and induction cores. Pulsed magnets are also being evaluated as a cheaper, more flexible option.

\section{SUMMARY}

The HCX experiment is being built to explore intense beam transport limits at a scale typical of the low kinetic energy end of a multi-beam HIF driver. A single, coasting beam is employed for economy. The experiment will be carried out in a phased implementation with Phase I (in assembly) testing ESQ transport, and Phase II (in planing) testing magnetic quadrupole transport. Later phases may test acceleration, bunch compression, and other issues.

\section{References}

[1] V. Karpenko et al., "An Engineering Overview of an Electrostatic Quadrupole Lattice for a High Current Transport Experiment," these proceedings.

[2] C. Celata et al., "PIC Simulations of Beam Dynamics Experiments on the HCX," these proc.

[3] S. Yu et al., "2MV Inj. as the Elise Front-End \& Exp. Facility," Fusion Eng. \& Des., 32-33 (1996) 309-315.

[4] F. Bieniosek et al., "2-MV Inj. for HCX," these proc.

[5] A.Faltens and P.Seidl, "Devel. of Elec. Quadrupoles for HIF," XVIIth Int. Symp. on Discharges and Elec. Insulation in Vac., July 1996. IEEE cat. \#96CH35939.

[6] D. Shuman et al., “A Pulsed Magnetic Quadrupole Array for HIF, "Proc. Part. Accel. Conf. (1999) p. 785.

[7] G. Sabbi et al., "Development of Superconducting Quadrupoles for HIF," these proceedings. 


\section{PROGRESS IN HEAVY ION DRIVEN INERTIAL FUSION ENERGY: FROM SCALED EXPERIMENTS TO THE INTEGRATED RESEARCH EXPERIMENT*}

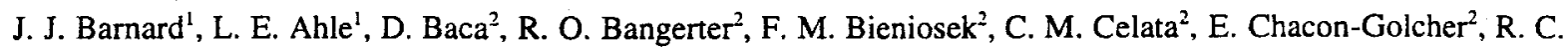
Davidson $^{3}$, A. Faltens ${ }^{2}$, A. Friedman', R.M. Franks'2, D. P. Grote', I. Haber, E. Henestroza', M. J. L. de Hoon'2, I. Kaganovich', V. P. Karpenko', R. A. Kishek ${ }^{3}$, J. W. Kwan'2, E. P. Lee', B. G. Logan', S.M. Lund', W. R. Meier', A. W. Molvik ${ }^{1}$, C. Olson ${ }^{7}$, L.R. Prost ${ }^{2}$, H. Qin ${ }^{3}$, D. Rose ${ }^{6}$, G-L. Sabbi ${ }^{2}$, T. C. Sangster', P. A. Seidl', W. M. Sharp ${ }^{1}$, D. Shuman', J.L. Vay², W.L. Waldron ${ }^{2}$, D. Welch ${ }^{6}$, S.S. $\mathrm{Yu}^{2}$

1. Lawrence Livermore National Laboratory, Livermore, CA; 2. Lawrence Berkeley National Laboratory, Berkeley, CA; 3. Princeton Plasma Physics Laboratory, Princeton, NJ; 4. Naval Research Laboratory, Washington, D.C.;

5. University of Maryland, College Park, MD 6.Mission Research Corporation, Albuquerque, NM 7.Sandia

National Laboratory, Albuquerque, NM

\section{Abstract}

The promise of inertial fusion energy driven by heavy ion beams requires the development of accelerators that produce ion currents $(\sim 100$ 's Amperes/beam) and ion energies $(\sim 1-10 \mathrm{GeV})$ that have not been achieved simultaneously in any existing accelerator. The high currents imply high generalized perveances, large tune depressions, and high space charge potentials of the beam center relative to the beam pipe. Many of the scientific issues associated with ion beams of high perveance and large tune depression have been addressed over the last two decades on scaled experiments at Lawrence Berkeley and Lawrence Livermore National Laboratories, the University of Maryland, and elsewhere. The additional requirement of high space charge potential (or equivalently high line charge density) gives rise to effects (particularly the role of electrons in beam transport) which must be understood before proceeding to a large scale accelerator. The first phase of a new series of experiments in the Heavy Ion Fusion Virtual National Laboratory (HIF VNL), the High Current Experiments (HCX), is now beginning at LBNL. The mission of the HCX is to transport beams with driver line charge density so as to investigate the physics of this regime, including constraints on the maximum radial filling factor of the beam through the pipe. This factor is important for determining both cost and reliability of a driver scale accelerator. The $\mathrm{HCX}$ will provide data for design of the next steps in the sequence of experiments leading to an inertial fusion energy power plant. The focus of the program after the HCX will be on integration of all of the manipulations required for a driver. In the near term following HCX, an Integrated Beam Experiment (IBX) of the same general scale as the HCX is envisioned.

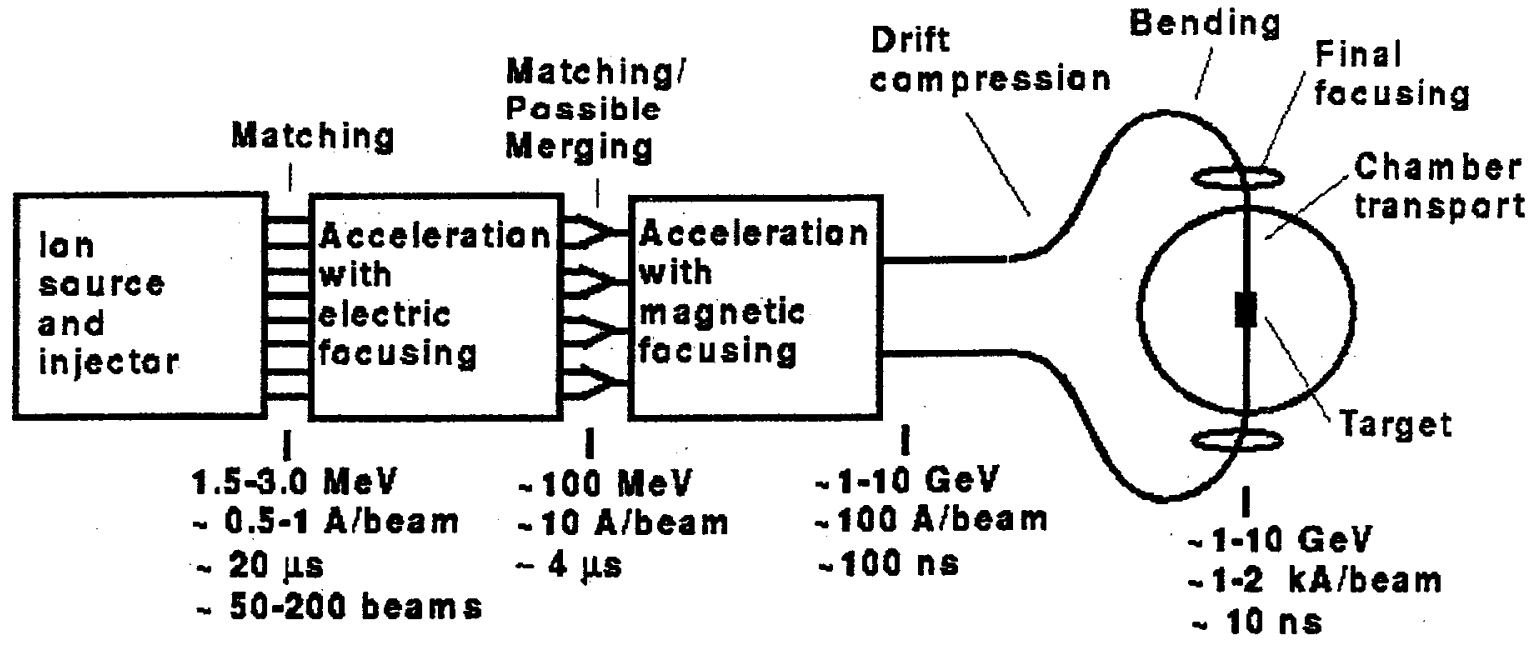

Figure 1. Schematic of the stages and beam manipulations required in one version of a Heavy Ion Fusion driver.

\footnotetext{
* This work was performed under the auspices of the U.S. Department of Energy under University of California contract W-7405-ENG-48 at LLNL, and University of California contract DE-AC03-76SF00098 at LBNL, and PPPL contract
} 
The step which bridges the gap between the IBX and an engineering test facility for fusion has been designated the Integrated Research Experiment (IRE). The IRE (like the IBX) will provide an integrated test of the beam physics necessary for a driver, but in addition will provide target and chamber data. This paper will review the experimental and theoretical progress in heavy ion accelerator driver research from the scaled experiments through the present experiments and will discuss plans for the IRE.

\section{INTRODUCTION}

Inertial fusion energy targets require the deposition of beam energy onto small spots, 2 to $5 \mathrm{~mm}$ in radius, at the ends of a hohlraum (indirect drive) or onto a spherical capsule (direct drive). The most detailed radiation/hydrodynamical simulations of heavy ion targets have been done for hohlraums in which the ions deposit their energy in converters. The converters radiate their energy in $\mathrm{x}$-rays, which impinge upon capsules, ablating and compressing them[1]. The total pulse energy required is -3 to $7 \mathrm{MJ}$, with a main pulse duration of $-8-10 \mathrm{~ns}$. The ion range required by the target is -.02 to $0.20 \mathrm{~g} / \mathrm{cm}^{2}$, which implies an ion energy of between $\sim 1$ to $10 \mathrm{GeV}$ for ion masses between $\sim 80$ and 200 . Final ion currents between 30 and $900 \mathrm{kA}$ are thus needed to meet the pulse energy requirement. The high currents are achieved by compressing the beam length by a factor of order 20 , so initial line charge densities between 15 and $900 \mu \mathrm{C} / \mathrm{m}$ are required. Quadrupole channels can transport a fraction of a $\mu \mathrm{C} / \mathrm{m}$ at typical injection energies. Comparing what is required at the target with the transportable current illustrates the need for multiple beams (10's to 100 's). Figure 1 illustrates the manipulations envisioned in a heavy ion fusion driver. These include injection of multiple beams, electric transport, a possible merge before magnetic transport, drift compression, beam bending, final focusing, and neutralized chamber transport .

The issues facing HIF can be broadly classified into two main groups: cost and focusability. The cost issue is continually being addressed both through studying component scaling imposed by the physics of beam transport and acceleration as well as through technology development projects required for the near term experiments (such as superconducting magnets, induction core materials, and insulators.) System studies are also used to determine high leverage machine architecture items affecting the overall cost of electricity.

The main scientific issue is focusability on the target. There are two main components which act to prevent focusability at the target: 1 . Space charge: Because currents are large, and because the chamber environment is envisioned to be filled with residual gas at the millitorr level, the mainline approach is to ionize the gas at the entrance to the target chamber. In addition, photoionization of the chamber gas by the beam heated target X-rays can be utilized. In both cases, the beam will draw electrons into its path to neutralize the space charge. Experiments and calculations are validating this concept.

2. Insufficient brightness: Over most of the beam path through the accelerator, economics dictates that space charge forces be much greater than thermal forces. At the target when the beam is focused down to a small spot thermal forces dominate (particularly when the beam is neutralized). It is thus important to maintain low emittance beams throughout, even when the emittance is not dynamically important. By making small, intense beams, target energy requirements can be reduced implying smaller, cheaper accelerators. So there is a big impact for getting the brightest beams possible.

\section{EXPERIMENTAL PROGRAM}

\subsection{Scaled Experiments[10]}

Two flagship experiments of the HIF program in the mideighties and early nineties began the process of demonstrating that the manipulations required for an induction linac driver can be carried out.

In the Single Beam Transport Experiment (SBTE) [3] (mid-1980's), the limits in undepressed and depressed phase advance were investigated. In contrast to some theoretical predictions of instabilities (using a KV distribution function), particle-in-cell simulations predicted and SBTE results then confirmed stable propagation for tune depressions (ratio of depressed to undepressed phase advance) as low as .1 to .2 (the limit achievable in the experiment). Undepressed phase advances greater than $\sim 85$ degrees were unstable for any significant tune depressions. Further, the experiment was at a length that was a significant fraction of the number of half-iattice periods in a driver $(-10 \%)$.

The Multiple Beam Experiment (MBE-4) [4] (late 1980's and early 1990's) provided an initial demonstration of acceleration, current amplification, longitudinal confinement using "ear" fields, and transport of multiple (i.e. four) beams.

A bending experiment at LLNL [5], demonstrated bending and acceleration using induction cells, sensing (using capacitive probes, even while firing the induction cells) and steering the beam. The original plan was to make the bend the beginning of a recirculating induction accelerator, but limited funding forced the program to concentrate on the mainline linac approach.

More recently [6] it was demonstrated that the four beams from MBE4 could be combined into a single beam, with an emittance growth that was expected on the basis of both theory and simulation.

Also, recently [7] the SBTE apparatus was again used to ballistically focus a beam using a one-tenth scale version of a final focus design from the power plant study called 
HIBALL II [28]. Electric quads were used to prepare the beam for entrance into a magnetic final focus system. A spot size consistent with space charge and emittance was produced. In a later version of the experiment a heated wire filament was placed in the beam path supplying the beam with neutralizing electrons. Simulations using the LSP code agreed well with the experiment [8].

Other scaled experiments include an adiabatic plasma lens [9], a channel transport experiment [9] and others [10]. including the University of Maryland electron beam experiments [11], which are highly relevant to HIF.

Some of the HIF experiments (past, present, and future) are summarized in Table 1. It can be seen from the table that nearly every major manipulation required in an HIF driver, has been carried out, at some level, in the scaled experiments.

\subsection{Driver Scale Experiments}

Presently, the HIF program is developing experiments in which the line charge density of the beam is at or near that expected for the early phase of a driver accelerator. Line charge density is important, because it determines the space charge potential drop of the beam from center to the pipe radius, and hence the confining potential for both unwanted electrons (in the accelerator) and wanted electrons (in the chamber). Hence, the present program is examining the science of the propagation of "driver-scale" beams.

One of the main focuses of the program is the High Current experiment (HCX) [12]. The first phase of the experiment (next $\sim 2$ years) is to transport a driver scale beam $\left(\sim 0.6\right.$ ampere and $\sim 1.8 \mathrm{MeV}$ of $\left.\mathrm{K}^{+}\right)$. The potential drop from beam center to pipe radius will be $-5 \mathrm{keV}$. This phase of the experiment will consist of 40 electrostatic quadrupoles followed by four magnetic to begin assessing magnetic transport. The second phase is currently planned to examine transport through 50-100 magnetic quads.
The beam filling factor $r_{\text {beam }} / r_{\text {pipe }}$ is important for obtaining a cost-optimized accelerator. Understanding the evolution of the emittance has strong implications for the target, and thus on overall cost. So the first phase of HCX will be to assess how close the beam can come to the pipe, by observing emittance growth, halo formation, and beam loss. The beam radius will be altered by changing the quadrupole voltages as well as the current. The question of optimum steering will be addressed, as well as the rate of electron production and entrainment. The role of desorbed atoms, born from beam halo particles hitting the walls or from ionized residual gas atoms accelerated by the beam space charge towards the wall, will be assessed. Pulse duration limits (within the bounds of the experiment) from head particle loss affecting tail propagation will be explored.

Phase 1 will use the existing ESQ injector and matching section. (The matching section reduces the radius of the beam out of the diode and injector, and transforms it from a circular to an elliptical beam). This will be followed by four 10 quad blocks. At the beginning of each block there will be a quad which slides out of the way, so that diagnostics such as slit-scanners or pepper-pots can be inserted into the beam path. Further, two of the quads in each 10 quad block can be displaced in $x$ and $y$, producing a dipole component, and allowing steering experiments that place the beam closer to the wall. The first four quads in each block allow independent control of their voltages so that the beam can be rematched if necessary or envelope oscillations intentionally induced to examine halo production. Finally, one quad in each block can be intentionally rotated by up to a few degrees to look at the effects of skew angles on envelope and emittance.

Many simulations have been carried out in support of both the phase I and phase II HCX [12], [13]. Examples include WARP code simulations of the non-linear multipole fields intrinsic to the prototype superconducting magnets, imperfectly aligned quadrupoles with small but finite rotation angles and displacements, finite initial

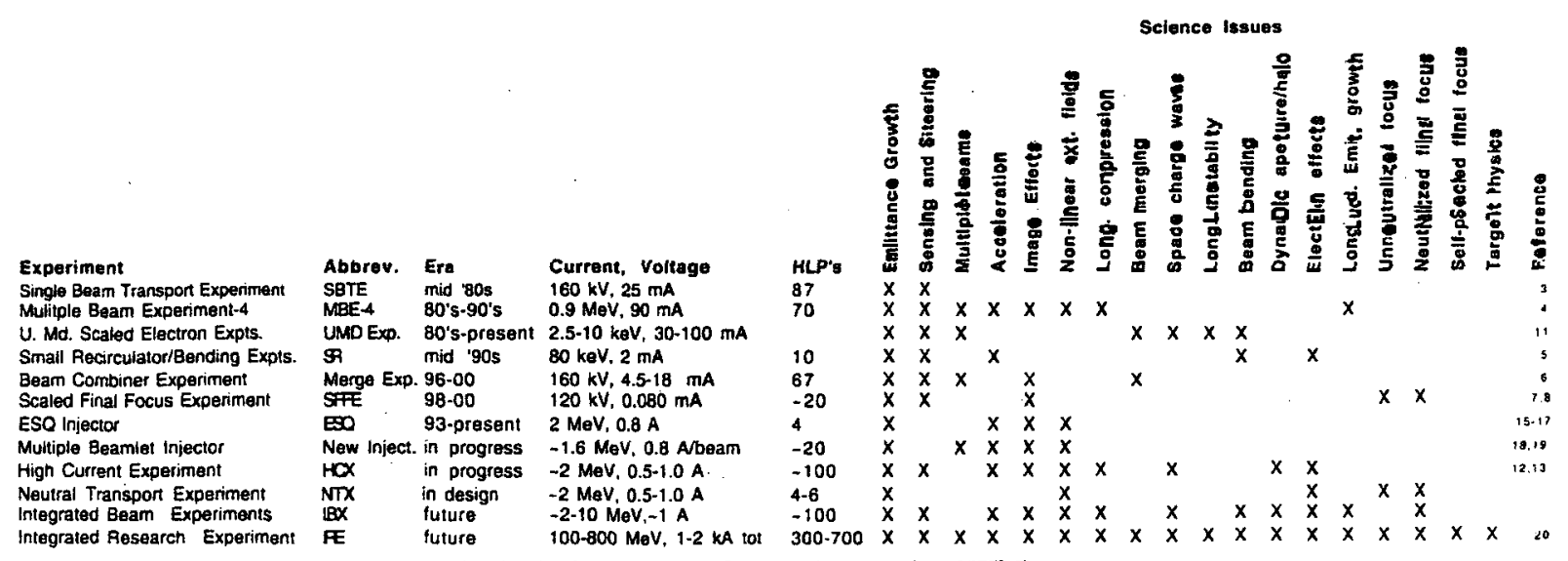

Table 1: Summary of Experiments in HIF Program 
displacements of the beam, and finite initial mismatches.

Several magnets are being designed for use in the HCX, (with the technology developed highly relevant to future accelerators including the Integrated Research Experiment). An array of 21 pulsed normal magnets was designed. An array which included 4 of these magnets has been pulsed approximately 80,000 times. Pulsed magnets would not be used in a driver (because the overall efficiency of the accelerator would not be acceptable for a power plant application) but, because of their cost advantage over superconducting magnets, pulsed magnets may be of value in an IRE or nearer-term experiment.

Two superconducting prototypes have been developed [14]. Both have circular apertures and square outer cross section, which would make them easily adaptable into a multi-beam array. One magnet uses rectangular Rutherford cables in a racetrack configuration of two layers, whereas the second group uses six layers of circular cables placed into grooves in a plastic matrix. Both prototypes have reached at least $90 \%$ of their theoretical maximum gradient, which is well above the requirements for the HCX. A selection of the baseline option will be made within a year.

The existing ESQ (which will be the front end to the HCX) has been found to have a current density distribution at its exit which is peaked near the edges[15]. The cause appears to be spherical aberration. Reduction of the radius of the source from $8.5 \mathrm{~cm}$ to $5 \mathrm{~cm}$ and modifications to the extraction electrode have, in simulations, reduced the nonuniformity and produced a more elliptical beam shape.

The other major VNL HIF experimental research project is design of a cost-effective injector. As pointed out earlier, multiple beams are required to transport the total charge required by the target. One way of doing this is to make a large diameter source that matches the maximum current limit of the ESQ transport channel. The ESQ Injector [17] is an example of this approach.

Another method, now being considered [18], is to merge hundreds of mm-scale beamlets into a single macro-beam. (There would still be tens to hundreds of macro-beams in the accelerator.) This merging-beamlet approach has the potential to reduce both the transverse and longitudinal dimensions of a multi-beam injector. The ChildLangmuir law for a diode relates the current density $J$ and voltage $\mathrm{V}$ across a gap of length $\mathrm{d}$ according to $\mathrm{J} \sim \mathrm{V}^{3 / 2} / \mathrm{d}^{2}$. But breakdown voltages are proportional to $\mathrm{d}$ (for short distances $<\sim 1 \mathrm{~cm}$ ) and roughly as $\mathrm{d}^{1 / 2}$ for larger distances $>-1 \mathrm{~cm}$ ). So $\mathrm{J} \sim \mathrm{V}^{-5 / 2}$ (small d) or $\mathrm{J} \sim \mathrm{V}^{-1 / 2}$ (large $\mathrm{d}$ ). In either case the current density increases as the voltage decreases, whereas the total current goes as $V^{3 / 2}$ (since $d$ scales with the radius of the source). The beam brightness $\sim$ current/emittance $^{2} \sim \mathrm{J} / \mathrm{T}$ where $\mathrm{T}$ is the source temperature; thus high current density translates to high brightness. Low $\mathrm{V}$ implies high current density but to get the required high current many beamlets $(-100$ 's per beam) are required. This approach is being both simulated using WARP and explored experimentally using a new $500 \mathrm{kV}$ source test stand now being completed at LLNL. [18], [19].

Because of the large radius of the low current density source, the beamlines in a multiple beam injector must converge as they proceed from the source to the transport region. To bend the beam gradually, and decrease the beam radius from source to transport region, a large distance is required to match the beam to the $\mathrm{ESQ}$ transport channel. With the multiple beamlet approach all of the beams can feed directly into the transport channel, reducing substantially both length and radius. Furthermore, the beams can start out elliptical, minimizing the manipulations, in the matching section. This would be especially attractive for an IRE, where the front end is a much larger fraction of the cost than it is for a driver.

The final VNL experiment, now in the design stage, is a "Neutralized Transport Experiment" or NTX, the purpose of which is to examine neutralized final focus of higher perveance beams. (This has been designed to operate at the end of the MBE-4 or at the end of the High Current Experiment). The experiment consists of a number of magnetic quadrupoles that form the final focus, followed by a short drift, corresponding to propagation in the chamber. An if source (now being readied at PPPL) ionizes the plasma, and so the effect of plasma at various locations within the chamber can be explored. This will be a flexible experiment allowing the experimental variation of plasma densities and gradients. Also, the large perveance planned for this experiment implies large final focusing angles, and therefore larger geometric aberrations. The question of whether octupoles can correct for the third order aberrations will be addressed experimentally on the NTX.

\subsection{Next Stage: Integrated Experiments}

After the HCX or as part of the later stages of HCX, the program envisions a near-term Integrated Beam Experiment $(\mathrm{IBX})$ in which nearly all of the components of a driver are put into place. This would include injection, acceleration, longitudinal compression, and final focus, with a driver scale beam. The focus is on integration and validation. In August 2001, a workshop will be held with the goal of systematically determining the science goals of IBX and beginning to scope out the accelerator parameters. The $\mathrm{IBX}$ will enable comparisons of experimental data with source-to-target simulations, using WARP in the accelerator and LSP in the chamber.

The IBX will lay the ground work for the single final step between itself and a demonstration Inertial Fusion Energy test facility. That intermediate step is known as the Integrated Research Experiment (IRE). 
The goals of the IRE go beyond just a thorough understanding of high-intensity beam physics [20]. The basic overriding principle is that, together with the knowledge gained in the DOE Defense Programs target physics program including the National Ignition Facility (NIF) and supporting technology programs, the IRE will give a basis to proceed to an IFE Engineering Test Facility. Several areas of target physics (particularly those unique to ions [as opposed to lasers]) will begin being explored. (It is possible that hydrodynamic motion during the initial "fpot" phase of the pulse could be investigated.) Examination of a variety of chamber transport modes, including self-pinched modes that are not accessible to the IBX, will be another major goal of the IRE. Further, chamber transport issues, particularly the interaction of beams with the liquid walls (Flibe) that are now favored to shield the solid walls of the target chamber, will be a research goal of the IRE. These goals dictate the scale of the facility to be - tenth scale of a driver in ion energy, and in the $30-300 \mathrm{~kJ}$ range in pulse energy.

In the meantime, our information about the IRE and drivers comes from simulations and theory. Recently there has been substantial progress made in simulations and theory of the accelerator, driver and drift compression section [21-25], as well as detailed simulations and theory of chamber transport [26, 27], which include a number of plasma neutralization scenarios.

\section{SUMMARY AND CONCLUSIONS}

The Heavy Ion Fusion IFE program is transitioning from scaled experiments to experiments with beams that are driver scale in line charge density and pulse duration. The main scientific issues for the driver are maintenance of high brightness beams, and production and focusing of a highly neutralized beam in the chamber. WARP3D simulations of the accelerator and LSP simulations of the chamber, together with perturbative $\delta f$ (BEST) simulations, theory and lower-dimensional simulations, explore and validate the physics of near-term experiments, a mid-term IRE and longer-term driver beams. The current experimental emphasis of the HIF program is on HCX, advanced injector concepts, and NTX Final Focus experiments, with the IBX and IRE to follow.

\section{REFERENCES}

[1] D. A. Callahan-Miller and M. Tabak, "Progress in Target Physics and Design for Heavy Ion Fusion." Phys. of Plasmas, 7, 2083 (2000). [2] W. Meier, A. Faltens, R. O. Bangerter, "An integrated systems model for heavy ion drivers," Nucl. Inst. and Meth. In Phys. Research, A., 415, 249, (1998).

[3] M.G Tiefenback, and D. Keefe, "Measurements of Stability Limits for a Space Charge Dominated Ion Beam in a Long A.G. Transport Channel," IEEE Transactions on Nuclear Science, NS-32, 2483 (1985).

[4] W.M. Fawley, T. Garvey, S. Eylon, E.Henestroza, A. Faltens, T.J. Fessenden, K. Hahn, and L. Smith, "Beam Dynamics Studies with the Heavy Ion Linear Induction Accelerator MBE-4," Phys. Plasmas 4. 880 (1997).

[5] L. Ahle et al, "Results from the recirculator project at LLNL,"Nuclear Instruments and Methods in Physics Research A, 464 557 (2001).
[6] P.A. Seidl et al, "Progress on the scaled beam combining experiments at LBNL," Nucl. Instr. and Methods in Physics Research A, 415, 243 (1998).

[7] S. MacLaren, E. Henestroza, A. Faltens, G. Ritchie, and P.Seidl, "Results from the Scaled Final Focus Experiment," Nucl. Inst. and Meth. in Phys. Res. A 464, 126, (2001).

[8] D. Welch and D. Rose, "Beam,Neutralization in the Scaled Final Focus Experiment," Proceedings of the 2001 Particle Accelerator Conference (RPAH068), (2001).

[9]A. Tauschwitz et al, "Plasma lens focusing and plasma channel transport for heavy ion fusion," Fusion Engineering and Design, 32-33, 493, (1996).

[10] P.A. Seid! et al,"Experiments at the Virtual National Laboratory for Heavy lon Fusion," )", Nucl. Inst. and Meth. In Phys. Research, A. 415, 369, (2001).

[11] M. Reiser, et al, "The Maryland Electron Ring for Investigation of Space Charge Dominated Beams in a Circular FODO lattice," Proceedings of the 1999 Particle Acclerator Conference, NY p. 234 (1999). Also P. Oshea et al, "The Univ. of Md. Electron Ring (UMER)", Nucl. Inst. and Meth. In Phys. Research, A., 415, 646, (2001).

[12] P. A. Seidl et al, "Overview of the Scientific Objectives of the High Current Experiment for Heavy-Ion Fusion," Proceedings of the 2001 Particle Accelerator Conference (RPAH037), (2001).

[13] ] C.M. Celata, D.P. Grote, I. Haber, "PIC Simulations of Beam Dynamics Experiments on High Current Experiment," Proceedings of the 2001 Particle Accelerator Conference (RPAH033), (200I).

[14] G-L Sabbi et al. "Development of Superconducting Quadrupoles for Heavy Ion Fusion," Proceedings of the 2001 Particle Accelerator Conference (RPPH088), (2001).

[15] F. Bieniosek, E. Henestroza, J.W. Kwan,"2 MV Injector for HCX," Proceedings of the 2001 Particle Accelerator Conference (WPAHO10), (2001).

[16] E. Henestroza et al. "Beam Dynamics Studies of the Injector and Matching Section for HCX," Proceedings of the 2001 Particle Accelerator Conference (RPAH035), (2001).

[17] S.S. Yu, S. Eylon, E. Henestroza, C. Peters, L. Reginato, A. Tauschwitz, D. Grote, and F. Deadrick. "2 MV injector as the Elise front-end and as an experimental facility," Fusion Engineering and Design 32-33, 317 (1996).

[18] J.W. Kwan et al,"Design of a Compact High Current Injector for Heavy Ion Fusion," Proceedings of the 2001 Particle Accelerator Conference (WPAH013). (2001).

[19] L. Ahle et al,"Initial Experiments of RF Gas Plasma Source for Heavy Ion Fusion," Proceedings of the 2001 Particle Accelerator Conference (FOACO05), (2001).

[20] J.J. Bamard et al,"Planning for a Heavy Ion Fusion Integrated Research Experiment," ," Nucl. Instr. and Methods in Physics Research A 464, 621 (2001)

[21]I. Haber et al, "Recent Progress in Heavy lon Fusion Simulations," "Nuclear Instruments and Methods in Physics Research A, 464, 343, (2001).

[22] A. Friedman et al, "Beam Simulations for IRE and Driver," "Nuclear Instruments and Methods in Physics Research A, 464, 653 (2001).

[23]M. de Hoon, E.P. Lee, J.J. Barnard,"Drift Compression of Space Charge Dominated Beams," Proceedings of the 2001 Particle Accelerator Conference (FOAC006), (2001).

[24] E.P. Lee and J.J. Barnard,"Design Strategies for Final Pulse Compression with Bending for Heavy Ion Fusion Drivers," Proceedings of the 2001 Particle Accelerator Conference (RPAH036), (2001).

[25] C. Jun et al, "Study of Drift Compression for Heavy Ion Fusion," Proceedings of the 2001 Particle Accelerator Conference (TPPH043), (2001).

[26] D.Welch et al,"Simulation of Self-Pinched Chamber Transport of Ions for Heavy Ion Fusion," Proceedings of the 2001 Particle Accelerator Conference (RPAH067), (2001).

[27] I. Kaganovich, "Nonlinear Charge and Current Neutralization for an Intense Ion Beam Puise Propagating Through a Background Plasma," Proceedings of the 2001 Particle Accelerator Conference (TPPH317), (2001).

[28] H. Wollnik et al., "HIBALL-II: An Improved Conceptual Heavy Ion Driven Fusion Reactor Study," KfK-3840, FPA-84-4, UWFDM-

625, Karlsruhe, July. 1985. 


\title{
COMPUTATIONAL INVESTIGATION OF DISSIPATION AND REVERSIBILITY OF SPACE-CHARGE DRIVEN PROCESSES IN BEAMS
}

\author{
R. A. Kishek ${ }^{\circledR}$, C. L. Bohn*, I. Haber ${ }^{\#}$, P. G. O'Shea, M. Reiser, and H. Kandrup ${ }^{\dagger}$, \\ Institute for Electronics and Applied Physics ${ }^{\ddagger}$, University of Maryland, College Park, MD 20742
}

Abstract

Collisionless charged particle beams are presumed to equilibrate via the long-range potential from the space charge. The exact mechanism for this equilibration, along with the question of macroscopic reversibility, has been uncertain, however. A number of computational approaches based on particle-in-cell (PIC) methods are presented which can facilitate the resolution of these questions. One such technique is the self-consistent tracking of individual particle orbits through the nonlinear potential formed by nonuniform charge density distributions: This orbit-tracking model differs from the particle-core model in that the sampled particles are systematically chosen from the actual particles in a fully self-consistent simulation. The results of this analysis are presented for a number of representative cases, and the implications of the study on equilibration mechanism are discussed.

\section{INTRODUCTION}

Charged particle beams with space charge are typically collisionless Hamiltonian systems where the detailed density distribution self-consistently governs the dynamics via Poisson's equation. The questions of equilibration, damping, and reversibility are of fundamental importance in determining beam properties. For example, equipartitioning of anisotropic beams involves nonlinear energy transfer and evolution towards an isotropic meta-equilibrium [1]. It is important for the accelerator designer to know whether the exchange of energy in this process is reversible. The same question arises when considering emittance growth due to misalignments or dispersion [2]. Reversibility implies the possibility of correction. Although extensive theory has been developed about thermodynamic equilibrium as it applies to beams [3], the absence of collisions necessitates the search for an alternative dissipation mechanism responsible for equilibration.

In this paper, we consider the possibility of one such dissipation mechanism: namely, phase mixing of globally chaotic particle orbits [4], which we call chaotic mixing. Chaotic orbits are characterized by sensitivity to initial conditions leading to exponential separation of nearby orbits. The process is therefore fundamentally irreversible. Further, globally chaotic orbits distribute themselves throughout their accessible phase space.

(a) ramiakốchre.umd.edu

* FNAL, Batavia, IL

\# Naval Research Lab, Washington, DC.

+ U. of Florida, Gainesville, FL.

\$ hutp:/iwww ireap.umd.edu/umer/
If chaos turns out to be an important contributor to the dynamics of beams, then (i) the physics of rapid "relaxation processes" can be accessed and studied, and (ii) the accelerator designer will need to account for it.

We have developed computational tools, based on the particle-in-cell (PIC) code WARP [5], which we are using to investigate the behavior of interacting or noninteracting test particles affected by the self-consistent potential of the beam. We give examples involving both chaotic and regular orbits, and relate the associated dynamics to observable phenomena such as damping and equilibration.

\section{COMPUTATIONAL TOOLS}

For the simulations, we use the $2-1 / 2 \mathrm{D}$ version of the PIC code, WARP. Large numbers of particles are pushed in the self-consistent potential created by the space charge, as well as a predefined external field. For these preliminary runs we apply only a constant linear external focusing ("smooth approximation"), so any nonlinearity in the fields arises solely from the non-uniformities of the particle distribution. The degree of nonlinearity in the system can be manipulated by introducing more sophisticated geometries, such as a mismatch, a periodic lattice, anisotropy, altering the initial distribution, or any of host of complications.

Collections of test particles are also introduced at various locations of phase space. Each set of test particles ("ensemble") initially occupies a narrow portion of the 4$D$ phase space, and is introduced in the code as a different species, albeit with the same charge and mass as the main beam, to ease in tracking it. The weight of macroparticles in each species can be adjusted at will. Thus we can make the test particles invisible to the main beam by setting the weight to zero; alternatively, we can give them any arbitrary non-zero weight, causing them to perturb the self-fields. The different ensembles are initialized to have a fraction of the size and velocity spread of the main beam, typically, $1 / 100$. The moments of each species (the main beam being species 0 ) are calculated and saved periodically. Further, a small number of particles are selected at random from each species and their trajectories saved.

For the cases shown here, a constant focusing strength of $k_{0}=15 \mathrm{~m}^{-2}$ is applied to a $10 \mathrm{kV}, 100 \mathrm{~mA}$ electron beam with an emittance of $50 \mathrm{~mm}-\mathrm{mr}$. This results in a beam radius of about $1 \mathrm{~cm}$, and a tune depression $\mathrm{k} / \mathrm{k}_{\mathrm{o}}$ of about 0.2 , making the beam highly space-charge- 
dominated. Typical numerical parameters are: $256 \times 256$ cells across the $5 \mathrm{~cm}$ diameter of the beam pipe; 4 million particles in the main beam (or 121 particles/cell); 20,000 particles in each test ensemble; and a step size of $2 \mathrm{~cm}$, significantly smaller than any of the characteristic wavelengths of the system $\left(\lambda_{\mathrm{p}}=1.14 \mathrm{~m} ; \lambda_{\beta \mathrm{o}}=1.63 \mathrm{~m}\right.$; $\lambda_{\beta}=12.6 \mathrm{~m}$ ). The initial distribution used in this case is a semi-Gaussian, although others will be attempted.

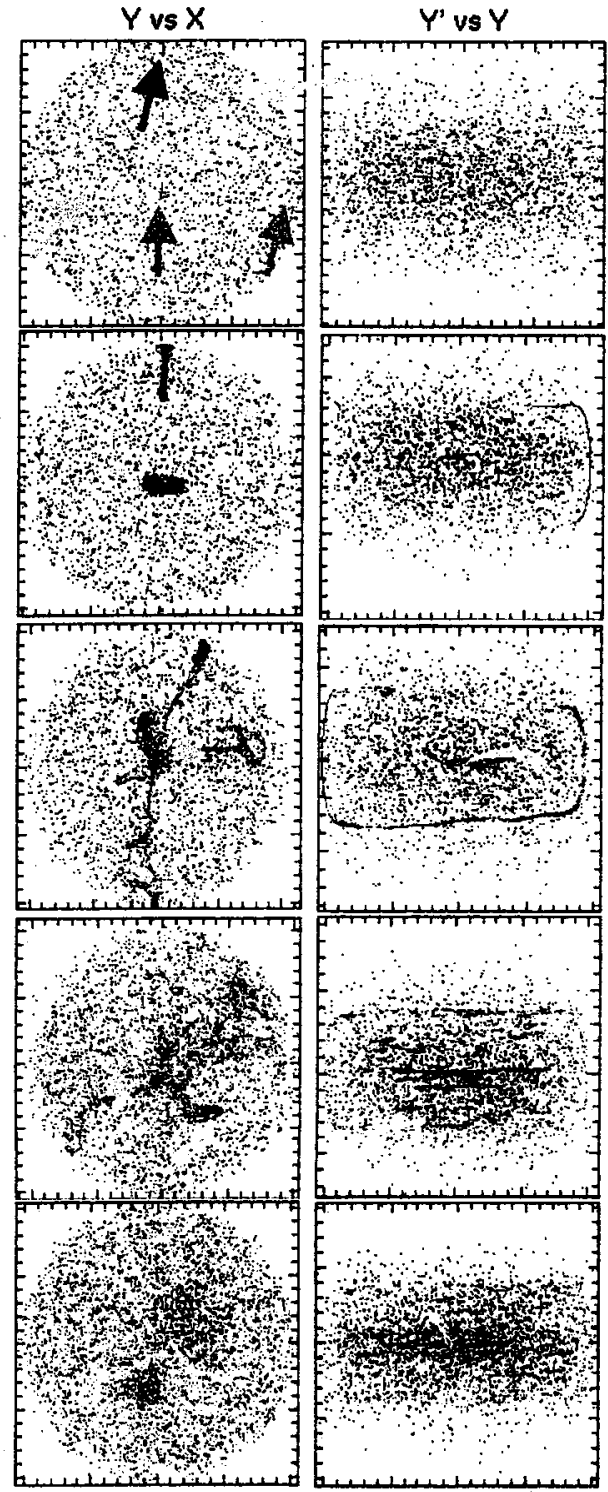

Fig. 1: Sequence of particle plots with colored ensembles, taken at distances of $0.0,11.52,51.84,100.8,169.9 \mathrm{~m}$ (top to bottom). Left is an $x-y$ projection, right is $y-y^{\prime}$.

\section{RESULTS}

Figure 1 demonstrates the growth of the size of each ensemble as a function of propagation distance, $s$. In this particular simulation, only the particles in the blue ensemble are interacting with the main beam (at full weight), while other ensembles are non-interacting. To ascertain whether the mixing is chaotic or not, we plot the moments of each ensemble on a semi-log scale, as shown in Fig. 2 for the "emittance" of the ensembles from the simulation of Fig. 1. It is apparent that for most ensembles, the exponential growth saturates at an early stage, before the particles fill even a fraction of the available phase space. Afterwards, the ensembles continue to grow in size, but not exponentially, indicating that the mixing mechanism is not globally chaotic. The small span of exponential growth at the beginning is an indication of local chaos, possibly arising from the particle noise. This is to be expected for a case like this, since the potential experienced by the particles is well behaved, and the only nonlinearities arise from relaxation of the particle distribution via small-amplitude space charge waves [6]. In this case, the blue ensemble has the fastest mixing rates, possibly because the blue ensemble acts as a perturbation to the potential in its vicinity.

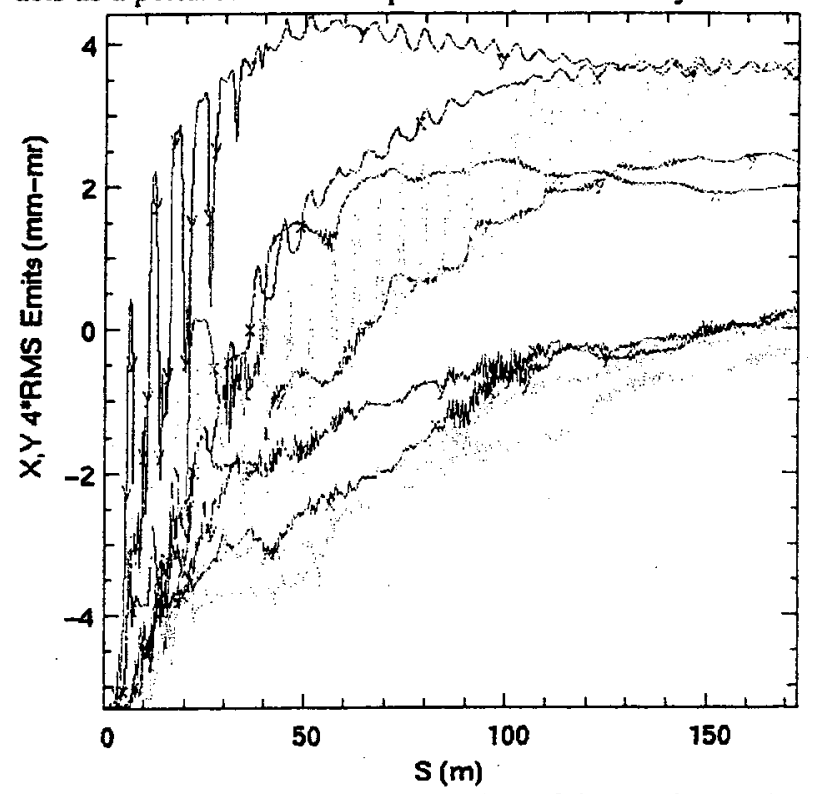

Fig.2: Evolution of natural logarithm of the "emittance" moment for ensembles in the isotropic beam of Fig. 1.

In order to observe more prominent mixing, we introduce an anisotropy by doubling the initial beam emittance in the $x$ direction to $100 \mathrm{~mm}$-mr [we further adjust the focusing field and the beam size to maintain a matched beam]. This anisotropy in a symmetric focusing channel has been observed [1] to very quickly lead to equipartitioning by exchange of energy in the two transverse directions. Figure 3 illustrates the growth of the emittance moment of each ensemble for this anisotropic case. Contrasting this with the curves from Fig. 2, it is obvious that the mixing proceeds much faster for all the ensembles in the anisotropic case. We further observe that the majority of ensembles now continue the exponential growth until they fill a large fraction of the phase space before saturating. This is a good indicator of chaotic mixing. 


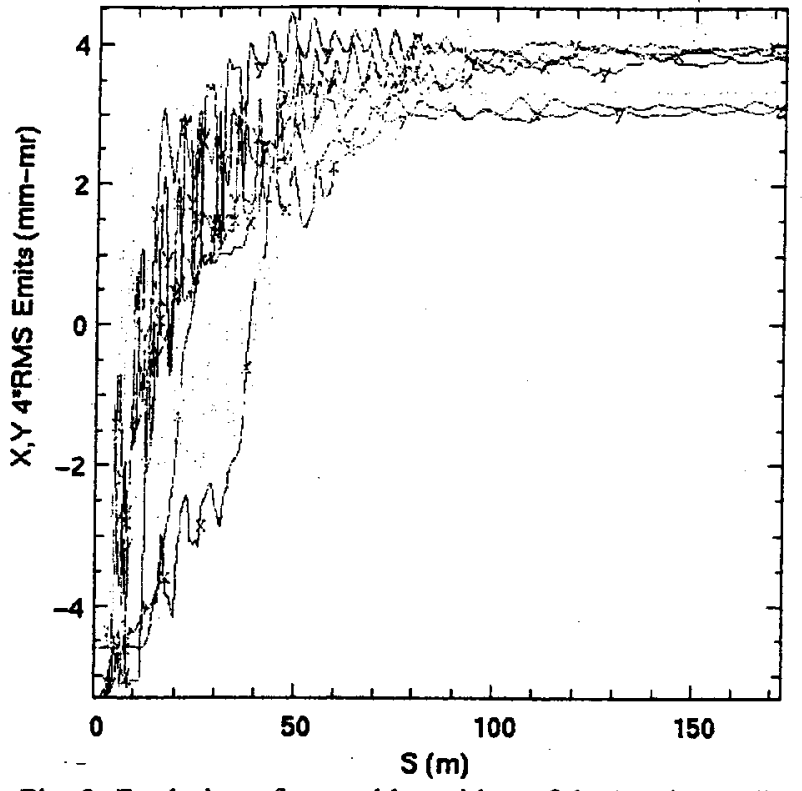

Fig. 3: Evolution of natural logarithm of the "emittance" moment for ensembles in the anisotropic beam.

The e-folding distance which can be measured from the figure is about $2 \mathrm{~m}$, roughly consistent with the scale over which the beam equipartitions (it takes about $5 \mathrm{~m}$ for the $\mathrm{x}$ and $y$ emittances of the main beam to become equal). It therefore appears that the chaotic mixing is somehow associated with the equipartitioning of the anisotropic beam. The total distance it takes for the ensembles to fill the entire phase space (about $50 \mathrm{~m}$ ) is furthermore consistent with the damping scale of the remaining oscillations in the main beam emittance.

We can draw another comparison by examining the particle orbits in phase space. Fig. 4 contains such plots for two distinct ensembles. The one on the left represents the trajectories of 20 particles from the red ensemble in the simulation in Figs. 1 and 2, whereas the one on the right is from the green ensemble in the simulation of Fig. 3. It is apparent that the orbits on the left are well behaved, whereas the ones on the right appear less regular and indicate possible chaotic behavior.

\section{CONCLUSIONS}

Preliminary results obtained so far indicate that there is a good possibility for chaotic mixing in beams. Much remains to be done to isolate real chaotic mixing from numerical effects and also to relate it to other phenomena such as instabilities. We wish to extend this investigation to more clearly delineate conditions under which chaotic mixing can take place. It is likewise conceivable that laboratory experiments in beams can be set up to study such physics with applications to other areas, such as galaxies [7], or large $\mathrm{N}$-body systems of self-interacting particles in general.

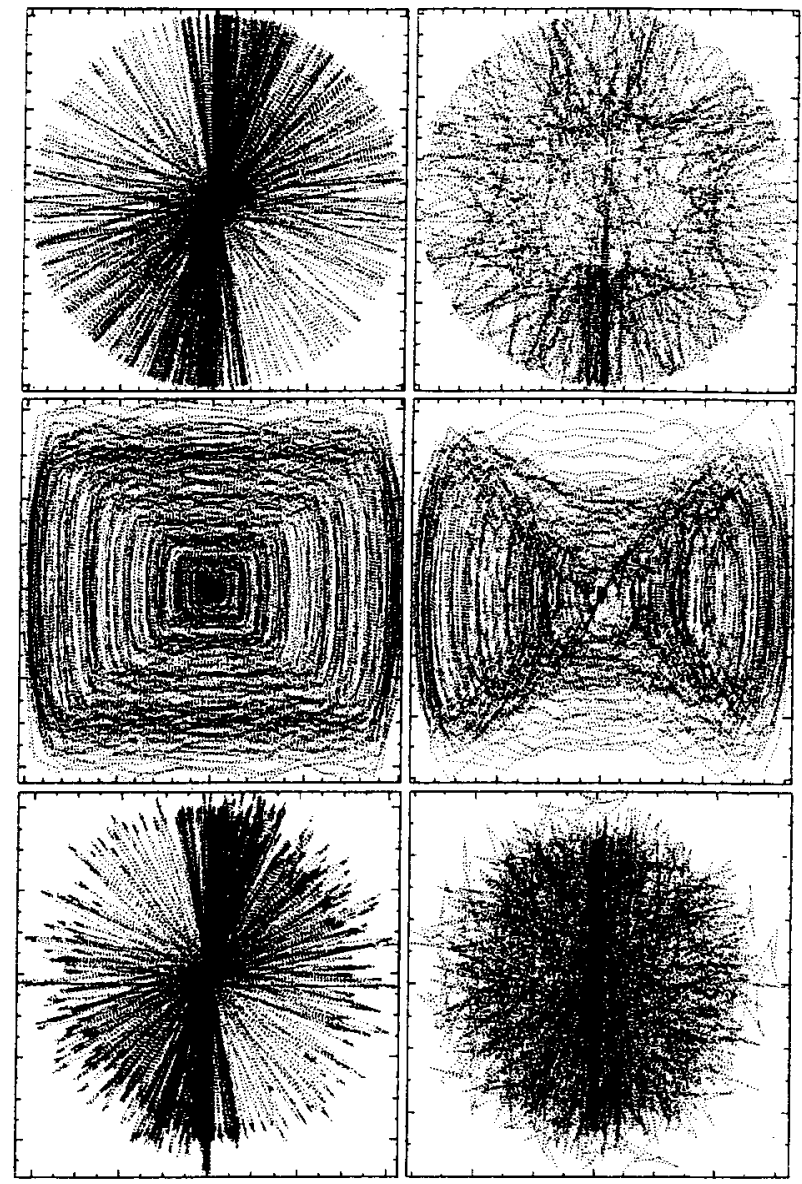

Fig. 4: Particle trajectories in phase space, $x-y, x-x^{\prime}$, and $x^{\prime}-y^{\prime}$ (top-bottom). 20 particles each from red ensemble of Figs. 1 and 2 (left); and green ensemble of Fig. 3 (right).

We thank Alex Friedman, Dave Grote, and Steve Lund for their excellent support of the WARP code. We further wish to thank S. Bernal and A. Valfells for valuable discussions. Research supported by US Dept. of Energy grant numbers DE-FG02-94ER40855 and DE-FG0292ER54178, and by Universities Research Association, Inc., under contract DE-AC02-76CH00300 with the U.S. Dept. of Energy.

[1] R. A. Kishek, P. G. O'Shea, and M. Reiser, Phys. Rev. Lett., 85 (21), 4514 (2000).

[2] M. Venturini, R.A. Kishek, and M. Reiser, PAC99, p. 3274 (1999).

[3] M. Reiser, Theory and Design of Charged particle Beams, (Wiley: 1994), chap. 5.

[4] C. L. Bohn, in The Physics of High Brightness Beams, (World Scientific, Singapore: 2000), p. 358.

[5] D. P. Grote, et. al., Fus. Eng. \& Des. 32-33, 193-200 (1996).

[6] S. Bernal, R. A. Kishek, M. Reiser, and I. Haber, Phys. Rev. Lett., 82, 4002 (1999).

[7] H. Kandrup, I. Pogorelov, and I. Sideris, Mon. Not. R. Astron. Soc., 319, 43-62 (2000). www.astro.ufl.edu/siopis/papers 